\title{
APLICAÇÃO DO MÉTODO DOS ELEMENTOS DE CONTORNO À PLACAS COM ENRIJECEDORES UTILIZANDO A TEORIA DE REISSNER
}

\author{
NEY AMORIM SILVA
}

Tese apresentada à Escola de Engenharia de São Carlos, da Universidade de São Paulo, como parte dos requisitos para obtenção do Título de Doutor em Engenharia Civil.

ORIENTADOR: Prof. Dr. Wilson Sérgio Venturini 
Silva, Ney Amorim

S581a Aplicação do método dos elementos de contorno à placas com enrijecedores utilizando a teoria de Reissner / Ney Amorim Silva. -São Carlos, 1996.

230p.

Tese (Doutorado) -- Escola de Engenharia de São Carlos Universidade de São Paulo, 1996.

Orientador: Prof. Dr. Wilson Sérgio Venturini

1. Placas (Estruturas). 2. Método dos elementos de contorno

I. Titulo. 
À minha esposa Amélia e aos meus filhos Izabela, Raquel e Gustavo dedico com amor e gratidão este trabalho.

Aos meus pais. 


\section{AGRADECIMENTOS}

Ao Prof. Dr. Wilson Sérgio Venturini pela amizade, compreensão e sobretudo pela orientação durante toda a realização deste trabalho

Aos colegas, professores e funcionários do Departamento de Estruturas da Escola de Engenharia de São Carlos - USP, pela amizade e convivio.

Ao Departamento de Engenharia de Estruturas da Escola de Engenharia da UFMG, pela confiança e oportunidade e à CAPES, através do programa PICD pelo apoio financeiro.

Aos pós-graduandos, César Lima Costa e Luiz Antonio de Souza, pela digitação e confecção dos desenhos.

Finalmente, aos amigos e familiares que sempre me deram apoio e incentivo, fundamentais à conclusão desta tese. 


\section{SUMÁRIO}

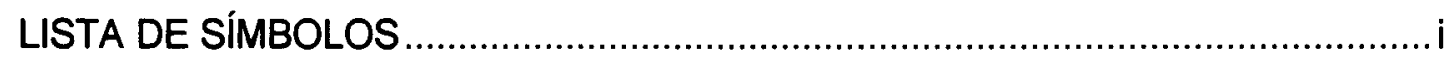

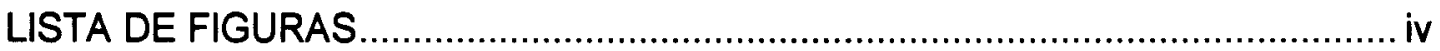

LISTA DE TABELAS …………………………………............................ vii

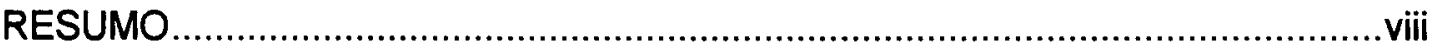

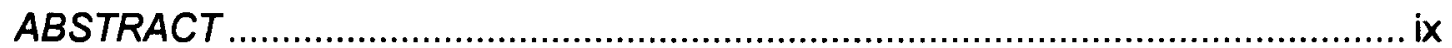

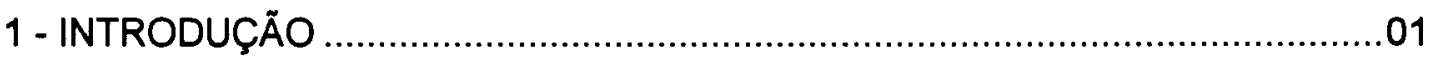

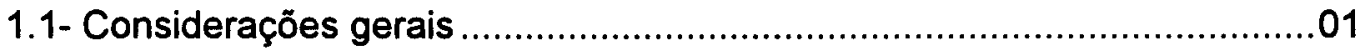

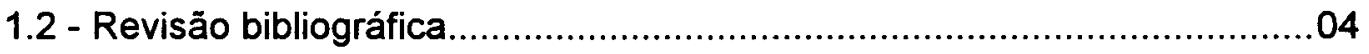

2 - TEORIA DE REISSNER APLICADA À FLEXÃO DE PLACAS .........................19

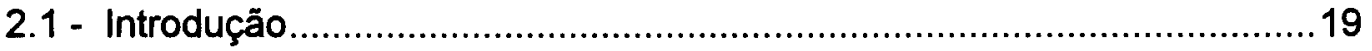

2.2 - Hipóteses básicas da teoria de reissner .............................................20

2.3 - Relações básicas da teoria de reissner .................................................

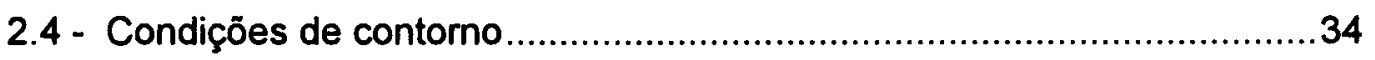

2.5 - Solução fundamental de placas usando a Teoria de Reissner................35

3 - ELEMENTOS DE CONTORNO APLICADOS À ANÁLISE DE PLACAS PELA

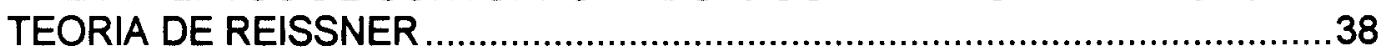

3.1- Introduçăo

3.2 - Equaçōes integrais de placas pela teoria de Reissner ............................39

3.2.1 - Equaçōes integrais para deslocamentos em pontos do domínio.....39

3.2.2 - Equações integrais para deslocamentos em pontos do contorno. ..45

3.2.3 - Forças fundamentais generalizadas no contorno ............................48

3.2.4 - Equaçōes integrais para esforços nos pontos do domínio. ..............51

3.2.5 - Cargas e momentos distribuídos no domínio....................................56

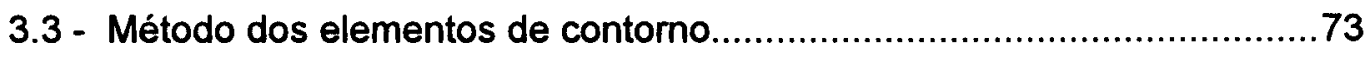

3.3.1 - Considerações iniciais .............................................................73

3.3.2 - Discretização das equaçōes integrais de contorno. ………….......74

3.3.3 - Influência do carregamento de domínio no cálculo dos valores de contorno

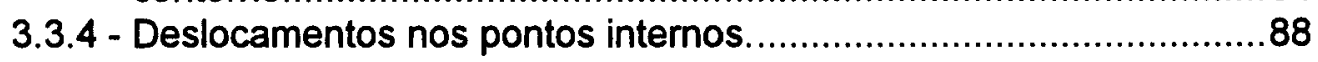

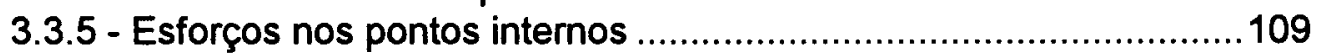

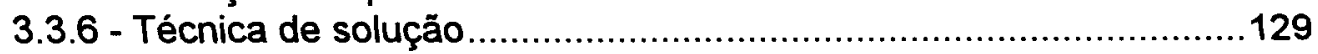


4.1- Introdução.

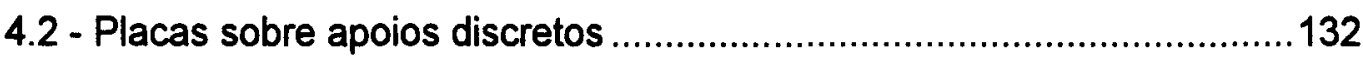

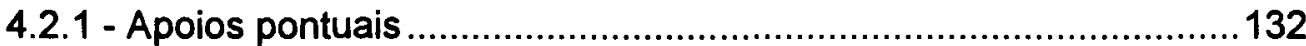

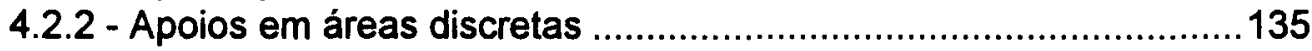

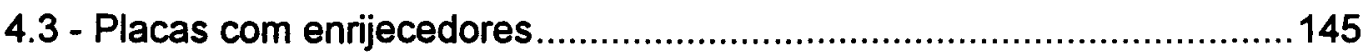

4.3.1 - Associação placa-estrutura qualquer...........................................145

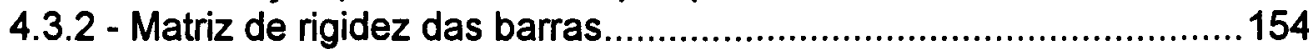

4.4 - Fluxogramas

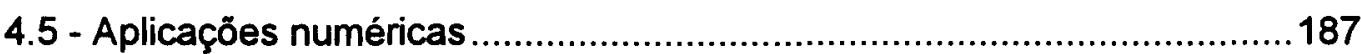

4.5.1 - EXEMPLO 1: Placa quadrada uniformemente carregada. .............187

4.5.2 - EXEMPLO 2: Placa alongada.................................................196

4.5.3 - EXEMPLO 3: Placa quadrada com apoios internos.....................197

4.5.4 - EXEMPLO 4: Placa com 6 apoios internos. ..................................200

4.5.5 - EXEMPLO 5: Placa com uma viga interna. ..................................203

4.5.6 - EXEMPLO 6: Placa quadrada apoiada em vigas elásticas. ............205

4.5.7 - EXEMPLO 7: Placa com duas vigas internas cruzadas. ................207

4.5.8 - EXEMPLO 8: Placa com duas e quatro vigas internas...................208

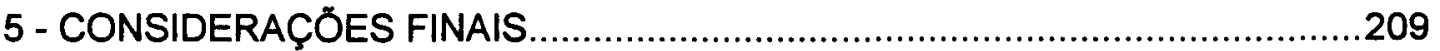

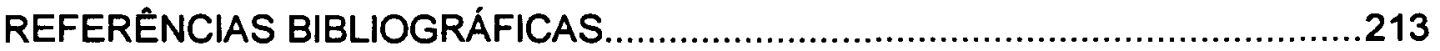

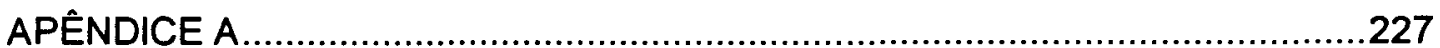




\section{LISTA DE SÍMBOLOS}

$x_{1}, x_{2}, x_{3} \quad$ - sistema de coordenadas cartesianas

$\sigma_{i j} \quad$ - componentes de tensão

w - deslocamento transversal da placa

$\phi_{1} \quad$ - rotação no plano $x_{1}-x_{3}$

$\phi_{2} \quad$ - rotação no plano $x_{2}-x_{3}$

$M_{\alpha \beta}$ - momentos fletores e de torção por unidade de comprimento

$Q_{3 \alpha} \quad$ - forças cortantes por unidade de comprimento

q - carga transversal aplicada à placa

$\mathrm{m}_{\alpha} \quad$ - momento distribuído aplicado à placa

$b_{i} \quad$ - forças de volume

h - altura da placa

$u_{i} \quad$ - deslocamentos da placa

$p_{i} \quad$ - forças de superfície

$U$ - energia de deformação / vetor que contém os deslocamentos dos pontos do contorno

P - vetor que contém as forças de superfície para pontos do contorno

$\eta_{i} \quad$ - cossenos diretores da normal ao contorno

$\Omega \quad$ - domínio da placa

$\Gamma \quad$ - contorno da placa

E - módulo de elasticidade longitudinal do material da placa

$v \quad$ - coeficiente de Poisson do material da placa

D - rigidez da placa à flexão

$\lambda$ - constante característica das equações de Reissner

$\nabla^{2} \quad$ - operador de Laplace

$u_{i j}{ }^{*} \quad$ - soluções fundamentais de placas em termos de deslocamentos

$p_{i j}^{*} \quad$ - soluções fundamentais de placas em termos de forças 
$\delta_{\mathrm{ij}} \quad$ - Delta de Kronecker

$\xi \quad$ - ponto fonte no domínio

$\chi \quad$ - ponto fonte no contorno

$x \quad$ - ponto campo no domínio

$X$ - ponto campo no contorno / vetor das incógnitas do contorno

$r \quad$ - distância entre os pontos fonte e campo

$r_{\alpha} \quad$ - componente de " $r$ " na direção " $\alpha$ "

$r_{, \alpha} \quad$ - derivada de " $r$ " na direção " $\alpha$ "

$\Omega_{q} \quad$ - subdomínio carregado com carga transversal " $q$ "

$\Omega_{m} \quad$ - subdomínio carregado com momento " $m_{\alpha}$ "

$\theta \quad$ - ângulo que define a direção de " $r$ " / rotação das extremidades dos elementos de barra

$\alpha \quad$ - ângulo que define a direção dos elementos de contorno / vetor dos parâmetros generalizados

$\beta \quad$ - ângulo entre a direção de "r" e a normal ao contorno

$\gamma$ - rotação devido à torção nas extremidades dos elementos de barra

$\eta \quad$ - coordenada local homogênea

$\phi_{\alpha}(\eta)$ - funções interpoladoras quadráticas

$l$ - comprimento do elemento de contorno

G - matriz de influência dos valores nodais/ módulo de elasticidade transversal

H - matriz de influência dos valores nodais

$g_{i j}, h_{i j}$ - componentes de "G" e "H"

B - vetor dos termos independentes

$k_{i j} \quad$ - coeficientes da matriz de rigidez

$\mathrm{K}$ - matriz de rigidez

$l_{x x}, l_{y y}-$ momentos de inércia 
S - área da seção transversal dos pilares / matriz que contém a influência da vinculação externa

Q - matriz que contém a influência dos apoios distribuídos em áreas

$k_{\text {o }} \quad$ - matriz de rigidez do elemento

$u_{e} \quad$ - deslocamentos nodais do elemento

$\checkmark \quad$ - vetor dos deslocamentos nodais dos elementos

F - vetor das forças nodais equivalentes / vetor resultante da influência dos valores prescritos no contorno mais os carregamentos de domínio

A - matriz contendo a influência dos valores incógnitos

$\mathrm{N}$ - matriz que transforma cargas nodais distribuídas em forças nodais equivalentes

$J_{t} \quad$ - momento de inércia à torção 


\section{LISTA DE FIGURAS}

Figura 2.1 - Sistema de coordenadas. Sentido das rotações 21

Figura 2.2 - Componentes de tensão no elemento genérico das placas .....22

Figura 2.3 - Momentos e cortantes resultantes 23

Figura 3.1 - Placa finita de espessura constante 40

Figura 3.2 - Placa com alteração do contorno no canto 46

Figura 3.3 - Área carregada $\Omega_{\mathrm{q}}$ 59

Figura 3.4 - Cargas distribuidas em subdomínios estreitos e alongados ....64

Figura 3.5 - Linha de carga com contorno alterado 66

Figura 3.6 - Discretização do contorno 75

Figura 3.7 - Elemento de contorno $\Gamma_{i}$ 75

Figura 3.8 - Colocação dos pontos fonte fora do domínio 82

Figura 3.9 - Divisão do elemento em sub-elementos 83

Figura 3.10 - Discretização do contorno da região carregada $\Omega_{q}$ 85

Figura 3.11 - Elemento de carga $\Gamma_{q}$ ou $\Gamma_{m} \operatorname{com} \xi=K_{2}$ 90

Figura 3.12 - Elemento de carga $\Gamma_{q}$ ou $\Gamma_{m} \operatorname{com} \xi=K_{1}$ 95 
Figura 3.13 - Elemento de carga $\Gamma_{q}$ ou $\Gamma_{m} \operatorname{com} \xi=K_{3}$ 98

Figura 4.1 - Placa sobre apoios pontuais. 132

Figura 4.2 - Associação placa-pilar. 138

Figura 4.3 - Conjunto placa-pilar. 139

Figura 4.4 - Área carregada $\mathrm{S}_{\mathrm{j}}$ 141

Figura 4.5 - Placa com uma linha genérica de cargas da interface 146

Figura 4.6 - Elemento genérico da ligação placa-estrutura 148

Figura 4.7 - Sistemas global e local de coordenadas. 155

Figura 4.8 - a) Sistema local de referência

b) Aproximação cúbica para v( $\eta)$

c) Aproximação linear para $\gamma(\eta)$ 156

Figura 4.9 - Elementos de carga e de barra. 164

Figura 4.10 - Placa simplesmente apoiada no contorno 188

Figura 4.11 - Reações no contorno para " $h / a=0,1 "$ 193

Figura 4.12 - Reações no contorno para " $h / a=0,01$ " 194

Figura 4.13 - Reações no contorno para "h/a=0,001" 195

Figura 4.14 - Placa alongada 196 
Figura 4.15 - a) Discretização em 4 e 24 elementos de contorno

b) Discretização em 9 e 225 elementos finitos 198

Figura 4.16 - Placa do Exemplo 3 200

Figura 4.17 - a) Discretização em elementos de contorno

b) Discretização em elementos finitos 201

Figura 4.18 - Discretização para o Exemplo 5

a) Placa com uma barras interna

b) Placa com quatro barras internas 203

Figura 4.19 - Discretização para o cálculo dos momentos 204

Figura 4.20 - Placa apoiada em vigas elásticas. 206

Figura 4.21 - Placa do Exemplo 7 207

Figura 4.22 - a) Placa com duas barras internas paralelas

b) Placa com quatro barras internas. 208 


\section{LISTA DE TABELAS}

Tabela 4.1 - Deslocamento transversal $\omega$ sobre a linha de centro da placa. 189

Tabela 4.2 - Valores do momento fletor $M_{x x}$ sobre a linha de centro da placa

Tabela 4.3 - Deslocamentos transversais $\omega$ e momento fletor $M_{x x}$ no centro da placa; momento de torção $M_{x y}$ no canto 192

Tabela 4.4 - Resultados do Exemplo 2 197

Tabela 4.5 - Deslocamento transversal " $w$ " 198

Tabela 4.6 - Momentos fletores 199

Tabela 4.7 - Deslocamentos verticais para o Exemplo 4 202

Tabela 4.8 - Momentos fletores para o Exemplo 4. 202

Tabela 4.9 - Deslocamento "W" no centro da placa. 203

Tabela 4.10 - Momentos fletores na viga 205

Tabela 4.11 - Resultados do Exemplo 6 206

Tabela 4.12 - Flecha e momento da viga para o Exemplo 7 207

Tabela 4.13 - Flecha máxima para as placas do Exemplo 8 208 


\section{RESUMO}

SILVA, N.A. Aplicaçăo do método dos elementos de contorno à placas com enrijecedores utilizando a teoria de Reissner. São Carlos, 1996. 219p. Tese (Doutorado) - Escola de Engenharia de São Carlos, Universidade de São Paulo.

Neste trabalho utiliza-se a formulação direta do Método dos Elementos de Contorno aplicada ao problema de flexão de placas com vinculação interna. Através da utilização da teoria baseada nas hipóteses de Reissner é possivel atender em cada ponto, a três condições físicas de contorno. Admite-se a aplicação de cargas transversais distribuídas e concentradas, além de momentos distribuídos em linha. Os elementos de contorno apresentam geometria linear com aproximação quadrática para as variáveis de contorno. As equações integrais dos deslocamentos são escritas para pontos de colocação dispostos fora do domínio, evitando-se assim problemas de singularidades. $O$ sistema de equações algébricas originado da análise de placas via MEC é modificado para incorporar o enrijecimento produzido pela vinculação interna. Primeiramente é analisado o enrijecimento produzido por apoios internos pontuais ou distribuídos em áreas pequenas e, em seguida, é estudada a associação da placa com uma estrutura qualquer, formada por barras. $O$ enrijecimento produzido por esta estrutura é obtido utilizando-se o Método dos Elementos Finitos. Finalmente, são apresentados alguns exemplos simples que mostram a boa precisão da técnica utilizada.

Palavras-chave: Placas; Método dos Elementos de Contorno 
SILVA, N.A. Analysis of stiffened plates through the Boundary Element Method employing Reissner's theory. São Carlos, 1996. 219p. Tese (Doutorado) - Escola de Engenharia de São Carlos, Universidade de São Paulo.

The direct formulation of the Boundary Element Method is applied to the analysis of internally restrained plates in bending. By employing Reissner's theory, the three boundary conditions are satisfied at each point. Loading conditions include concentrated or uniformly distributed loads as well as linearly distributed moments. The boundary elements are geometrically linear with quadratic approximation for the boundary variables. The displacement integral equations are written for collocation points outside the domain thus avoiding any singularity problem. The resulting system of algebraic equations is modified to include the stiffening effects. Plates with internal point restraints and restrained over small areas are analyzed as well as plates connected to others structures made up of bars. In this last case, the stiffening effect is calculated through the Finite Element Method. The results obtained in simple problems indicate the accuracy of the procedure.

Keywords: Plates; Boundary Element Method 


\section{INTRODUÇÃO}

\section{1- CONSIDERAÇÕES GERAIS}

As placas são elementos estruturais largamente utilizados em quase todos os ramos da engenharia, notadamente na engenharia civil, onde podem ser encontradas nos pisos e forros dos edifícios, nos tabuleiros das pontes, nos reservatórios, nos arrimos e cortinas de contenção, entre outros. A análise das placas constitui uma área de grande interesse tanto para projetistas como para pesquisadores que vem estudando 0 comportamento das mesmas, submetidas às mais variadas condições de carregamento e geometria. Dentro da Teoria da Elasticidade a "Teoria das Placas" constitui um dos tópicos mais importantes sob o ponto de vista das aplicações em engenharia, salientando-se que esta teoria faz uma aproximação do problema real tridimensional para um problema bidimensional.

A grande maioria dos problemas de engenharia é governada por equações diferenciais, cujas soluções analíticas só são conhecidas para alguns problemas particulares clássicos, restando aos demais a aplicação de métodos numéricos, que utilizam modelos discretos em substituição aos modelos contínuos dos métodos analíticos. $O$ estudo das placas através de métodos numéricos vem merecendo nas últimas três décadas a atenção de um grande número de pesquisadores. 
Com o aparecimento dos computadores tornou-se possivel e prático a utilização de métodos numéricos para análise de problemas físicos, que geralmente envolvem grandes sistemas de equações. Pode-se dizer portanto que o avanço da eletrônica, com o surgimento do computador, propiciou de forma inequívoca a utilização em grande escala dos métodos numéricos.

Os primeiros métodos numéricos, como o Método das Diferenças Finitas - MDF [1] e o Método dos Elementos Finitos - MEF [2,3,4], utilizam técnicas que discretizam o domínio. Apesar da dificuldade existente no Método das Diferenças Finitas com relação à discretização de contornos irregulares, ele é ainda utilizado para a solução de alguns problemas de engenharia. $O$ Método dos Elementos Finitos tornou-se nos últimos trinta anos o método numérico mais conhecido e utilizado em todos os ramos da engenharia [5], com convergência e eficiência comprovadas.

Mais recentemente surgiu o Método dos Elementos de Contorno MEC, assim chamado por utilizar equações integrais de contorno obtidas a partir das equações diferenciais do problema analisado. Este método vem experimentando um grande avanço nos últimos tempos devido a sua melhor adaptacão para determinados problemas especificos, como as regiōes de concentração de tensōes e regiōes infinitas. Outro fator muito importante para este crescimento é a redução da dimensăo do problema, uma vez que a discretização é feita apenas no contorno.

Este trabalho emprega o Método dos Elementos de Contorno [MEC], para análise de flexão de placas utilizando a Teoria de Reissner, que por considerar as deformações transversais devido ao cisalhamento é uma teoria mais refinada que a Teoria Clássica de Kirchhoff. A Teoria de Reissner possibilita $O$ atendimento de três condições físicas em cada ponto do contorno, desaparecendo desta forma as reações de canto necessárias para o estabelecimento do equilíbrio na Teoria Clássica, que atende apenas à duas condições de contorno. 
Diferentemente da Teoria Clássica a formulação apresentada com as hipóteses de Reissner, permite avaliar a influência da espessura nas respostas da placa, bem como considerar condições de contorno relativas à rotação no plano vertical tangente ao contorno.

Faz-se no início do trabalho uma revisão bibliográfica sobre as teorias de placas e o desenvolvimento de métodos numéricos que permitem respostas para as diversas aplicações na engenharia. No capítulo II são apresentadas as equações básicas para flexão de placas utilizando-se as hipóteses da Teoria de Reissner.

É apresentada no capítulo III a formulação integral de placas pela Teoria de Reissner. Inicialmente formula-se a representaçăo integral dos deslocamentos para pontos do domínio, estendendo-se depois esta representação para pontos do contorno. As representações integrais para esforços nos pontos internos são obtidas a partir das representações integrais dos deslocamentos correspondentes, aplicando-se a Lei de Hooke integrada na espessura da placa. São analisados também os termos integrais de domínio correspondentes ao carregamento aplicado em áreas, linhas ou concentrado em pontos.

Ainda no capitulo III o contorno é discretizado em elementos onde săo aproximados deslocamentos e esforços. Com isto as equações integrais de contorno deduzidas anteriormente são transformadas em um sistema de equaçōes algébricas lineares.

No capítulo IV analisam-se placas com enrijecimento interno e no contorno. Inicialmente são analisadas placas com vinculação interna proveniente de apoios que podem ser pontuais ou distribuidos em uma área. Estes apoios internos são tratados com rigidez axial real ou infinita, desprezando-se ou não a rigidez à flexão. É estudado também o enrijecimento proveniente das barras, que podem ser vigas de contorno ou internas. As vigas são analisadas como linhas de carga e o enrijecimento proveniente da estrutura formada pelas barras é obtido através do Método dos Elementos Finitos. Ao final deste capítulo são apresentados exemplos 
que formam com suas placas e barras, um piso de uma edificação. Os resultados são comparados com os obtidos com outros métodos numéricos.

\section{2 - REVISÃO BIBLIOGRÁFICA}

Nas últimas três décadas várias técnicas numéricas surgiram em função da dificuldade antiga de se obter soluções analíticas exatas para os problemas de engenharia, e principalmente devido ao avanço no campo da eletrônica, com o aparecimento do computador, que possibilitou de forma precisa e veloz a manipulação de grande massa de dados envolvida na análise destas técnicas.

Podemos citar como os principais métodos numéricos o Método das Diferenças Finitas, o Método dos Elementos Finitos e o Método dos Elementos de Contorno.

Os dois primeiros são denominados métodos de domínio por aproximarem a solução da equação diferencial do problema utilizando-se valores das variáveis envolvidas, associadas a pontos do domínio e do contorno analisados. No Método dos Elementos de Contorno a partir da equação diferencial do problema são deduzidas equaçōes integrais que são aproximadas utilizando-se valores discretos das variáveis básicas do problema em pontos do contorno apenas.

O Método das Diferenças Finitas [MDF] surgiu no início deste século através do trabalho de RUNGE e posteriormente com 0 trabalho de SOUTHWELL [1]. Este método consiste na transformação do sistema de equações diferenciais em um sistema algébrico, através da aplicação de operadores diferenciais que aproximam cada uma das derivadas parciais envolvidas, usando expansões locais, geralmente séries de Taylor 
truncadas, para representar as variáveis em sub-regiōes previamente definidas.

A grande característica associada ao Método dos Elementos Finitos [MEF] consiste em subdividir o domínio em uma série de elementos. São escolhidas funções aproximadoras capazes de representar os valores das variáveis em um ponto qualquer do domínio em função dos valores associados aos nós do elemento ao qual pertença o ponto. Cada elemento é equacionado individualmente e posteriormente são agrupados para a solução do problema como um todo. A formulação do método é geralmente apresentada a partir de principios variacionais, embora se obtenha uma maior generalização quando a formulação é apresentada utilizando-se a técnica dos resíduos ponderados. A utilização destas técnicas conduz a um sistema algébrico de equações, cuja matriz de coeficientes é simétrica e em forma de banda. Esta característica vem orientando nos últimos tempos várias técnicas visando a otimização da solução do sistema de equações. Este método é sem dúvida mais eficiente que o Método das Diferenças Finitas e tem sido largamente utilizado em todos os ramos da engenharia.

Atualmente a maioria dos programas computacionais comerciais ou acadêmicos utiliza o Método dos Elementos Finitos. Um dos problemas encontrados na aplicação desta técnica numérica é a necessidade de um alto grau de refinamento na discretização do domínio, quando se deseja obter um nível adequado de precisão, como por exemplo no caso de problemas com concentração de tensões. Nestes casos tem-se um grande volume de dados com um consequente aumento no número de equações.

Uma técnica relativamente nova do ponto de vista de aplicações computacionais foi denominada Método dos Elementos de Contorno [MEC], a partir do trabalho de BREBBIA [6]. Este método consiste na obtenção de equações integrais sobre o contorno a partir das equações diferenciais que governam o comportamento do corpo em seu domínio. As variáveis básicas do problema são calculadas em pontos discretos apenas no contorno. Quando necessário, as variáveis em pontos do domínio são obtidas 
diretamente a partir das variáveis do contorno. Destaca-se portanto como vantagem imediata da aplicação deste método, a redução em uma unidade da dimensão do problema. Isto implica em um menor volume de dados de entrada com uma redução no número final de equações do sistema. Outra característica do MEC é ter a matriz do sistema cheia e não apresentar simetria. Outros aspectos importantes do método devem ser citados como a facilidade de se modelar adequadamente os domínios infinitos, a ausência de erros de interpolação para pontos do domínio e a representação apropriada para problemas de concentração de tensōes [7].

Quanto a sua formulação, o Método dos Elementos de Contorno pode ser enquadrado basicamente em dois tipos: métodos indiretos e diretos. Os métodos indiretos foram os primeiros a aparecerem e são assim chamados por apresentarem a solução em função de variáveis fictícias associadas ao contorno. As variáveis físicas do problema são obtidas indiretamente a partir dos valores calculados para as variáveis fictícias, que não têm significado físico real e são obtidas a partir das condições de contorno em um certo número de pontos.

Nas formulações diretas mais utilizadas atualmente, as incógnitas das equações integrais são as próprias variáveis físicas do problema real. Desta forma nos problemas de elasticidade ,por exemplo, os deslocamentos e forças de superfície no contorno são obtidos diretamente da solução do sistema de equações. Os deslocamentos e esforços em pontos do domínio serão determinados, quando necessário, a partir das variáveis de contorno previamente calculadas. Conforme BREBBIA et al. [8] a formulação direta do Método dos Elementos de Contorno, baseada na técnica dos resíduos ponderados, tem a mesma origem dos demais métodos numéricos, podendo ser classificado como mais um método pertencente aos métodos aproximados. Esta é uma visão atualizada deste método que inicialmente teve sua formulação baseada em princípios clássicos, como o teorema de BETTI. 
Embora o Método dos Elementos de Contorno tenha aparecido recentemente, as equações integrais segundo ELLIOT [9], já são conhecidas desde 1823, quando $A B E L$ [10] deduziu uma equação integral para resolver o problema denominado Pêndulo Isócrono [11].As equações integrais experimentaram um avanço decisivo com o estudo dos problemas de potencial, destacando-se VOLTERRA [12], que estudou em 1884 a distribuição de cargas elétricas na superfície de uma esfera. Em 1903 FREDHOLM [13] demonstrou que podem ser obtidas soluçōes para equações integrais e posteriormente provou a existência e unicidade de tais soluções, na forma dos Teoremas de Fredholm. Estes estudos influenciaram muitos trabalhos posteriores, como os realizados por HILBERT [14], entre 1904 e 1910. Ainda sobre problemas de potencial deve-se citar o trabalho de KELLOG [15].

Historicamente, segundo LOVE [16], o método envolvendo equações integrais foi primeiramente aplicado na teoria clássica da elasticidade por BETTI em 1872. Trabalhos posteriores nesta área foram completados por SOMIGLIANA por volta de 1880 . Nesta mesma época CERRUTI aplicou o método para problemas de elasticidade plana [17].

Após este período, foram realizados outros estudos por matemáticos russos, entre eles MUSKHELISHVILI [18], que aplicou as equações integrais para resolver problemas de elasticidade linear bidimensional. MUSKHELISHVILI [18], MIKHLIN [19] e KUPRADZE [20] utilizaram equações integrais singulares baseadas na teoria das variáveis complexas [11]. A formulação indireta foi utilizada nestes trabalhos além de outros como os realizados por MASSONET, OLIVEIRA, WATSON e VAN BUREN (ver BANERJEE [21]). JASWON [22,24] e SYMM [23], introduziram em 1963, um equacionamento do problema usando variáveis reais, além de manter uma função de tensão auxiliar, caracterizando o que alguns autores [21] chamam de método semi-direto.

RIZZO [25] em 1964 apresentou um método para elasticidade plana relacionando variáveis reais do contorno, isto é, deslocamentos e esforços. 
Seguindo esta formulação direta CRUSE [26], estendeu este método para elasticidade tridimensional. Nestes trabalhos a aproximação das variáveis no contorno é feita por uma função constante. A aproximação linear para tais variáveis em problemas de duas e très dimensões, foi introduzida respectivamente por RICARDELLA [27] e CRUSE [28]. LACHAT [29], desenvolveu posteriormente elementos com aproximações de ordem superior.

A primeira aplicação para problemas de flexão em placas utilizandose as equaçōes integrais foi introduzida por JASWON et al. [30], que propôs a solução da equação biharmônica, via equação integral e posteriormente aplicou-a na solução de placas [31].

ALTIERO \& SIKARSKIE [32], apresentaram uma técnica que consiste em considerar a placa real contida em uma placa fictícia, cuja função de GREEN é conhecida. Posteriormente WU \& ALTIERO [33] estenderam esta técnica para incluir condições arbitrárias de contorno. Um trabalho similar foi desenvolvido por TOTTENHAN [34], que também apresentou uma formulação integral para cascas abatidas.

A formulação direta para placas foi simultaneamente introduzida por BEZINE [35,36] e STERN $[37,38]$. Bezine apresentou uma formulação integral usando elementos constantes e seus resultados se restringiram ao estudo de cargas concentradas. Stern desenvolveu uma formulação integral geral, mas não levou em conta a possibilidade da descontinuidade das condiçōes de contorno nos nós de canto.

Destacam-se outros trabalhos para a análise dos mais variados problemas de placas, como BEZINE [39], KAMIYA [40,41], TANAKA [42]. WEEËN [43] desenvolveu uma formulação para placas espessas, baseada na teoria de REISSNER $[44,45,46]$.

A aplicação do MEC para resolver problemas de placa sobre fundação elástica é um campo que tem direcionado ultimamente muitos pesquisadores. O primeiro trabalho nesta área foi desenvolvido por TOTTENHAN [34] em 1979. KATSIKADELIS \& ARMENÀKAS [47,48] 
apresentaram duas formulaçōes diferentes para analisar placas sobre fundação elástica, baseadas na teoria de WINKLER [49]. Mais recentemente citam-se os trabalhos de COSTA JR. \& BREBBIA $[17,50,51,52]$. Embora nestes últimos trabalhos as integrais de domínio para as cargas já tenham sido transformadas em integrais sobre o contorno, a resposta do solo é sempre elástica. Nos trabalhos de SILVA \& VENTURINI $[53,54]$ o domínio é discretizado em células onde são aproximados a reação do solo e seus incrementos, quando é assumida a não-linearidade para a resposta do solo. O processo da reciprocidade dual [55] é introduzido na formulação, como uma alternativa para a não discretização do domínio.

O MEC tem sido aplicado a materiais com comportamento não-linear $[27,56,57,58,59]$. TELLES \& BREBBIA [60], propuseram a resolução de problemas elastoplásticos e visco-plásticos introduzindo no equacionamento, esforços e deformaçōes iniciais. VENTURINI [61,62] aplicou o método na área da mecânica dos solos, analisando comportamento plástico, visco-plástico e materiais rochosos, sem resistência à tração. Recentemente outros trabalhos tratando a nãolinearidade tem sido desenvolvidos $[63,64,65]$.

Alguns autores tem estudado a combinação do MEC com outras técnicas numéricas, principalmente o MEF [66] a [77], [6] e [8]. Esta combinação tem como finalidade, utilizar o método numérico mais apropriado para cada região do corpo em estudo, de forma a se obter resultados mais precisos com redução no número de operações e o mínimo de tempo de processamento.

Ao longo do tempo diversas técnicas de análise de placas foram desenvolvidas sempre adotando simplificações, visando transformar o problema real, tridimensional, em um problema bidimensional. Dentre estas, as mais utilizadas nos trabalhos e pesquisas são a Teoria Clássica de Kirchhoff e a Teoria de Reissner.

A Teoria de Kirchhoff [78] analisa placas delgadas com pequenos deslocamentos baseando-se nas seguintes hipóteses: 
- a placa é constituída de material elástico-linear homogêneo.

- os deslocamentos transversais são pequenos se comparados à espessura da placa.

- uma reta inicialmente normal ao plano médio, permanece reta e perpendicular ao plano médio deformado, após a flexão da placa. Isto equivale a não considerar as deformações por cisalhamento transversal.

- as tensões normais à superfície da placa são pequenas em relação às tensões normais de flexão, paralelas ao plano da placa, podendo ser desprezadas.

- admite-se que a placa seja constituída de material homogêneo, isotrópico, cujo comportamento é elástico linear.

Estas hipóteses levadas à formulação das placas conduzem a uma equação diferencial de quarta ordem.

A Teoria de REISSNER [44] considera o efeito das deformações por cisalhamento transversal o que leva a um sistema de equações diferenciais de sexta ordem. Com este sistema é possivel e necessário atenderem-se três condições de contorno ao longo das bordas, ao invés de apenas duas, como estabelece a Teoria Clássica de Kirchhoff. A omissão da energia de deformação por cisalhamento transversal, segundo REISSNER [46], é responsável pela reduçăo das três condições físicas de contorno em apenas duas. O problema tratado sem esta omissão conduz a resultados significativamente mais precisos que os da Teoria Clássica, para o caso de pontos situados próximo às bordas e para pontos situados em volta de furos, cujos diâmetros sejam da mesma ordem de grandeza da espessura da placa.

MINDLIN [79] deduziu um modelo muito próximo ao de Reissner, para o movimento transversal de placas fletidas elásticas e isotrópicas. Este estudo é baseado nas equações de equilíbrio da elasticidade tridimensional para um corpo em movimento, considerando-se a inércia rotacional e 0 cisalhamento transversal. O sistema de equações diferenciais obtido é também de sexta ordem. 
Estudando os problemas relativos à integração para o caso de ortotropia e à influência de uma faixa limite de condições de contorno reduzidas, REISSNER [80] conseguiu distinguir os efeitos da deformação por cisalhamento, associadas à faixa de contorno e ao interior da placa. Isto foi obtido usando-se a solução para placas isótropas em função da flecha e de uma funçăo de tensão.

SALERNO \& GOLDBERG [81] reduziram o sistema de três equações diferenciais de Reissner a uma equação diferencial de quarta ordem semelhante à da Teoria Clássica, e a uma equação diferencial de segunda ordem, para determinação de uma função de tensão. Os resultados obtidos para placas simplesmente apoiada, mostraram, quando comparados com a Teoria Clássica, bons resultados.

As diversas teorias disponiveis para análise de placas são tratadas de forma abrangente por PANC [82]. Considerando as deformações por cisalhamento, LEVINSON [83], apresentou uma nova formulação considerando análise estática e dinâmica de placas com espessura constante. Esta nova formulação, para o caso dinâmico, conduz à mesma equação de onda para deslocamento transversal, da teoria de Mindlin.

Uma nova formulação apresentada por REISSNER [84], pode ser imaginada como uma generalização das equaçōes utilizadas na teoria de placas para grandes deslocamentos. O sistema de equaçōes obtido é de décima ordem, sendo duas equações simultâneas de quarta ordem e uma equação de segunda ordem. Para placas moderadamente espessas, REISSNER [85] apresentou em 1987 uma formulação que leva a um sistema de equações diferenciais de décima segunda ordem, mostrando resultados coerentes com a teoria clássica. Este mesmo autor em 1991 analisou sistematicamente o problema da influência de faixas de contorno para placas ortotrópicas [86]. "Neste trabalho é estudado o conceito de apoio "soft", como uma condição para transição suave da teoria de sexta ordem para a teoria de quarta ordem"[140]. 
Alguns trabalhos estimam o erro a partir da teoria clássica de placas em relação à teoria exata de um problema correspondente na teoria da elasticidade, entre eles, NORDGREN [87,88], RYCHTER [89,90].

Uma versão melhorada da Teoria de Reissner para placas homogêneas, isotrópicas e com quaisquer condições de contorno, foi proposta por LADEVĖZE \& PECASTAINGS [91], cuja diferença estava relacionada às condições de contorno e ao fator de deformabilidade por cisalhamento transversal, considerado na Teoria de Reissner.

O problema de placas submetidas a um carregamento transversal foi tratado por BARRET \& ELLIS [92] como uma perturbação singular. Estes autores relacionaram sua teoria com as teorias de Kirchhof, Mindlin e Reissner.

Muitas pesquisas sobre flexão de placas foram e continuam sendo desenvolvidas utilizando o Método dos Elementos Finitos [MEF]. As primeiras abordaram a Teoria de Kirchhoff e buscavam funções aproximadoras contínuas de classe $\mathrm{C}^{1}$, isto é, funções aproximadoras com derivadas primeiras contínuas, necessárias à obtenção de elementos conformes.

A utilização de elementos finitos triangulares para análise de placas data do início da década de sessenta, estando descrito de uma forma bastante abrangente até 1984 o estado da arte sobre este assunto, nos trabalhos de BATOZ et al. [93] e de HRABOK \& HUDEY [94]. A busca de um elemento triangular conforme, em termos de deslocamentos, orientou os esforços dos pesquisadores, que de uma maneira geral propuseram elementos conformes muito rígidos, necessitando um maior número de pontos de integração, quando comparados aos não-conformes.

BAZELEY et al. [95] obtiveram excelentes resultados com a utilização de dois elementos triangulares $\mathrm{BCIZ1}$ e $\mathrm{BClZ2}$, utilizando-se coordenadas homogêneas e as primeiras idéias do "patch test". 
PIAN [96] desenvolveu uma formulação alternativa, utilizando o princípio da energia complementar mínima, dando origem ao método hibrido.

Em geral os elementos hibridos dão resultados melhores que os elementos conformes obtidos em termos dos deslocamentos.

Uma outra formulação apresentada inicialmente em 1968 e seguida por diversos pesquisadores, consiste em adotar-se 0 modelo em deslocamentos usando-se as hipóteses de Kirchhoff de forma discreta, ao longo dos lados dos elementos ou em determinados pontos. Devido à sua complexidade, esta formulação só se tornou atraente, mostrando bom desempenho a partir do elemento DKT ("Discret Kirchhoff Triangle") proposto por BATOZ et al. [93,97] em 1980.

$O$ desenvolvimento de elementos de placa experimentou $O$ avanço mais expressivo quando a Teoria de Kirchhoff foi substituída pela Teoria de Reissner. Mesmo tendo que considerar o cisalhamento na formulação, a exigência de continuidade do tipo $\mathrm{C}^{0}$, para as funçōes aproximadoras dos deslocamentos, foi sem dúvida o fator decisivo deste avanço, uma vez que as derivadas primeiras, são as de grau máximo que aparecem no funcional obtido com a nova teoria. Foi detectado nos primeiros elementos de placas de Reissner o fenômeno do travamento, relacionado à preponderância dos coeficientes devido ao cisalhamento na matriz de rigidez, no caso de placas com pequena espessura. Para evitar este problema foram criados esquemas de integração reduzida e seletiva. De acordo com HUGHES [98], estes esquemas podem levar em certos casos à problemas de singularidades na matriz de rigidez. Isto foi superado com sucesso com a utilização de elementos triangulares propostos por TESSLER \& HUGHES [99], PAPADOPOULOS \& TAYLOR [100] e por ZIENKIEWICS \& LEFEBVRE [101]. Estudos realizados por HÄGGBLAD \& BATHE [102] consideravam as hipóteses da Teoria de Reissner e visavam obter resultados confiáveis nos pontos próximos às bordas e aos cantos da placa. 
Bergan e seus colaboradores, mantiveram as hipóteses de Kirchhoff em conjunto com funções de forma altamente não conformes. Segundo RIBEIRO [140], eles demonstraram que a continuidade entre elementos não é um obstáculo à convergência, desde que as funções de forma satisfaçam às condições de ortogonalidade entre força e energia. A matriz de rigidez é obtida a partir da formulação livre proposta por BERGAN \& HANSSEN [103] ao contrário da formulação padrão, pela energia potencial. Uma particularidade interessante nesta formulação é a separação cuidadosa entre as funçōes de deslocamentos básicas assumidas e as funções ou modos de ordem superior. A convergência é garantida dependendo da maneira como ocorre o acoplamento entre estas funções. Nesta mesma linha devem ser destacados os trabalhos de BERGAN \& NYGARD $[104,105,106]$ e de FELIPPA \& BERGAN $[107,108]$. Esta formulação embora apresentada inicialmente como uma formulação bastante diferente das anteriores, está relacionada à formulação padrão em deslocamentos via energia potencial, e ao modelo híbrido, conforme mostrado em [104] e [108], respectivamente.

O trabalho inicial de análise de placas utilizando o Método dos Elementos de Contorno é devido à JASWON et al. [30], publicado em 1967. Este trabalho propõe a decomposição da equação bi-harmônica em duas equações harmônicas que transformadas em equações integrais e devidamente combinadas, permitem a solução final. Com este trabalho inicia-se o desenvolvimento do método na aplicação à engenharia de estruturas, relativo às placas.

Em 1976, HANSEN [109] apresentou uma formulação direta para análise de placas infinitas com furos de contorno não carregado, usando duas equaçōes integrais, uma representando o deslocamento transversal e a outra a sua derivada, em relação a uma direção qualquer.

BEZINE \& GAMBI [36] propuseram em 1978, uma formulação direta partindo da identidade de Green, considerando como variáveis os deslocamentos transversais e sua derivada na direção normal ao contorno, 
que são as variáveis físicas do problema real, ou os valores correspondentes à força cortante equivalente e ao momento fletor normal ao contorno. Esta técnica foi aplicada à diversas placas quadradas com diferentes condições de contorno.

BEZINE [35] e STERN [37] desenvolveram trabalhos usando a formulação direta e teoria de Kirchhoff.

ALTIERO \& SIKARSKIE [32] e WU \& ALTIERO [33], usaram a formulação indireta para placas, resolvendo exemplos práticos apenas para contornos engastados, devido às dificuldades encontradas para outros tipos de contorno. Nestes trabalhos, uma placa engastada de contorno circular, de solução conhecida, é utilizada como contorno auxiliar.

TOTTENHAN [34] apresentou uma discussão sobre as formulações direta e indireta, estendendo ao caso de placas apoiadas sobre base elástica e cascas abatidas.

GUO-SHU [110], HARTMANN \& ZOTEMANTEL [111], adotaram esquema de interpolação hermitiana para a flecha e discutiram o tratamento das integrais de domínio, a consideração de vínculos no domínio e as singularidades que ocorrem na formulação direta via Teoria de Kirchhoff.

Adotando ainda as hipótese de Kirchhoff, PAIVA [113] utilizou várias alternativas para análise de placas, ora usando equações integrais para flecha e sua derivada primeira na direção normal ao contorno, ora usando duas equaçōes de flecha para dois pontos singulares, um no contorno e outro fora do domínio. Esta análise foi estendida para problemas práticos de engenharia, visando contemplar associação da placa com vigas e pilares.

HARTLEY e seus colaboradores estudaram basicamente os problemas envolvendo as singularidades que aparecem nos integrandos e a determinação de valores para pontos internos, $[114,115,116]$.

A eficiência e vantagens do Método dos Elementos de Contorno para análise de placas, baseadas na Teoria Clássica, enfatizadas por HARTMANN [117], levaram-no a sugerir que o mesmo supera o Método dos Elementos Finitos para este tipo de problema. 
Vários trabalhos foram publicados nos últimos tempos tratando de placas finas como PILTNER \& TAYLOR [118], KATSIKADELIS \& ARMENÀKAS [119], SAPOUNTZAKIS \& KATSIKADELIS [120], VITOORAPORN \& MOSHAIOV [121].

O equacionamento de placas apoiadas sobre base elástica foi inicialmente abordado por TOTTENHAN [34] em 1979, tendo como base as hipóteses de Winkler e Kirchhoff. Nesta mesma linha surgiram os trabalhos de KATSIKADELIS \& ARMENÀKAS $[47,48]$, COSTA JR. \& BREBBIA [50,51,52], BEZINE [122], SILVA[53].

KATSIKADELIS \& KALLIVOKAS [123,124] retomando o estudo de placas sobre base elástica adotou o modelo biparamétrico de Pasternak para simular a ligação solo-placa. Em 1991 KATSIKADELIS [125] introduziu na formulação do problema a ocorrência de grandes deslocamentos, tendo como base as equações de Von Kármán, permitindo comportamento nãolinear entre o deslocamento e a resposta do solo.

Outro problema importante é a análise de placas com não-linearidade geométrica, devido à ocorrência de grandes deslocamentos. Este assunto de grande interesse para a engenharia de estruturas foi tratado por TANAKA [126], KAMIYA \& SAWAKI [127] e YE \& LIN [128]. O trabalho de TANAKA [126] analisou placas finas elásticas com grandes deslocamentos e apresentou uma formulação integral e incremental equivalente às equações de Von Kármán, enquanto que o trabalho de KAMIYA \& SAWAKI [127] baseou-se na equação de Berger. Podem-se citar outros trabalhos nesta área como os divulgados por SAWAKI et al. [129], KATSIKADELIS \& NERANTZAKI [130], KAMIYA et al. [131], e KAMIYA [132].

O problema envolvendo vibrações livres e forçadas em placas finas elásticas foi apresentado por PROVIDAKIS \& BESKOS [133,134]. AKKARI \& HUTCHINSON [135] além de estudarem vibrações em placas finas, estenderam suas análises, contemplando placas espessas.

A não-linearidade física em placas, envolvendo deformação lenta, plasticidade e fratura, foi objeto de muitos estudos realizados por 
MUKERJEE \& MORJARIA $[59,136,137,138]$. A análise do comportamento elastoplástico de placas finas via Kirchhoff foi desenvolvida por MOSHAIOV \& VORUS [139], usando um esquema de carregamento incremental, com a consideraçăo de momentos fletores plásticos iniciais, calculados por um processo iterativo. A placa é dividida em células internas, onde as componentes do momento plástico são admitidas constantes. RIBEIRO [140] atuou nesta mesma área considerando campos de momentos iniciais no domínio da placa, permitindo-se com isto a análise de efeitos de gradientes de temperatura e retração além da consideração do comportamento não-linear do material.

A instabilidade de placas sujeitas à cargas no seu próprio plano foi analisada por COSTA JR [141] e também por BEZINE [142], utilizando-se células internas para o cálculo da integral de domínio.

A análise de placas através da Teoria de Reissner foi inicialmente tratada por WEEËN $[43,143]$ em 1982, admitindo comportamento elástico e enfatizando as vantagens das hipóteses adotadas, que levam a um sistema de equaçōes diferenciais de sexta ordem. Para cada ponto do contorno foram escritas três equações integrais cujas variáveis são os deslocamentos generalizados (rotaçōes normal e tangencial, e a flecha), e os respectivos esforços (momentos normal e tangencial e força cortante). O contorno é aproximado com elementos isoparamétricos quadráticos. KARAM [144], em 1986, usando como referência os trabalhos de Weeën demonstrou a eficiência do método através de vários exemplos de placas isotrópicas em regime elástico linear. RIBEIRO \& VENTURINI [145] seguindo a formulação de WEEËN $[43,143]$ tomaram os pontos de carga fora do domínio, evitando com isto algumas singularidades. Os trabalhos de BARCELLOS \& SILVA [146] e WESTPHAL \& BARCELLOS [147] abordaram também a flexão de placas usando as hipóteses de Reissner, identificando funções livres e funções essenciais como componentes da solução fundamental. A nãolinearidade geométrica devido a ocorrência de grandes deslocamentos 
analisada sob a teoria de Reissner foi formulada por XIAO-YAN et al. [148] em 1990.

A combinação do Método dos Elementos de Contorno com o Método dos Elementos Finitos, para problemas de flexão em placas foi apresentado em 1990 por NG et al. [149].

A aplicação do Método dos Elementos de Contorno à sólidos elásticos tridimensionais foi primeiramente analisada por CRUSE $[26,28]$ e LACHAT [29], já citados anteriormente. Estes trabalhos representaram importantes avanços da utilização do método a sólidos tridimensionais, mas são anteriores à denominação atual do método.

NAKAGUMA [150] apresenta uma formulação direta do MEC para problemas de elasticidade tridimensional, baseada nas soluções fundamentais de Kelvin, Mindlin e Boussinesq-Cerruti.

CUROTTO [151] apresenta uma formulação para elasticidade tridimensional partindo do teorema da reciprocidade de Betti, utilizando a solução fundamental de Kelvin, considerando efeitos de forças de volume e temperatura.

SÁ \& TELLES [152] utilizam um algoritmo similar ao de Nakaguma, com as expressões de Mindlin apresentadas de forma compacta. A utilização das soluções fundamentais de Mindlin e Boussinesq-Cerruti por Nakaguma na aplicação de interação solo-estrutura ou escavações traz grande versatilidade ao método, ao dispensar a discretização da superfície do solo segundo BARBIRATO [153].

RIZZO [25], LACHAT [29], CRUSE [26] e BREBBIA [6] utilizam a solução fundamental de Kelvin enquanto BANERJEE [154], BUTTERFIELD \& BANERJEE [155], utilizam a solução de Mindlin. A solução de Boussinesq-Cerruti, versão tridimensional da solução de Flamant, é vista em LOVE [16]. 


\section{TEORIA DE REISSNER APLICADA À FLEXÃO DE PLACAS}

\section{1 - INTRODUÇÃO}

Os elementos estruturais podem ser classificados como lineares, de superfície e tridimensionais, conforme tenham respectivamente duas, uma ou nenhuma dimensão pequena quando comparada às outras.

Dentre os elementos estruturais de superfície destacam-se as placas, por serem bastante utilizadas nas estruturas usuais. A placa é definida como um corpo limitado por duas superfícies planas. A distância entre estas superfícies, denominada espessura, é pequena quando comparada às outras dimensōes. A superfície eqüidistante das superfícies limites é denominada superfície média, ou no caso de placa plana, plano médio.

Neste trabalho serão consideradas apenas placas planas submetidas a carregamentos verticais, transversais ao plano médio.

Dependendo das propriedades do material, as placas são classificadas em anisótropa - com propriedades diferentes em qualquer direção; ortótropa - com propriedades diferentes em duas direções ortogonais, e isótropa - com propriedades iguais em todas as direções.

Dependendo da espessura, as placas podem ser classificadas como muito delgadas, delgadas e espessas. Segundo MARTINELLI et al. [157], a teoria clássica de Kirchhoff interpreta suficientemente bem o comportamento 
das placas delgadas, cuja relação h/a (espessura/menor das dimensỏes do plano da placa) está entre os limites $1 / 100<(\mathrm{h} / \mathrm{a})<1 / 5$. A formulação para as placas muito delgadas ( $\mathrm{h} / \mathrm{a}<1 / 100)$ deve incorporar a ocorrência de grandes deslocamentos e baseia-se nas equaçōes de Von Kárman. A teoria clássica de Kirchhoff não permite avaliar o efeito da espessura sobre os valores das flechas e esforços, considerando a placa como delgada. A teoria mais refinada de Reissner permite tal avaliação e pode ser tanto empregada para placas delgadas como espessas $(\mathrm{h} / \mathrm{a}>1 / 5)$. Segundo RIBEIRO [158], a faixa de variação h/a, dentro da qual é válido considerar a placa como delgada depende entre outras coisas, fundamentalmente da variável de comparação escolhida, isto é, deslocamentos ou esforços.

Neste trabalho será considerado apenas a análise de placas delgadas utilizando-se as hipóteses da Teoria de Reissner.

\section{2 - HIPÓTESES BÁSICAS DA TEORIA DE REISSNER}

A determinação das relações e equações básicas, necessárias para a formulação do problema de flexão de placas segundo Reissner, baseia-se nas seguintes hipóteses:

- A placa é constituída de material elástico linear homogêneo.

- A espessura é pequena quando comparada com as outras duas dimensões no plano da placa.

- Uma reta inicialmente normal à superfície média indeformada, permanece reta após a deformação da placa, mas não necessariamente normal à superfície média deformada, devido a consideração das deformações por cisalhamento transversal. 
- As componentes tangenciais da tensão são nulas nas faces da placa.

- As tensões normais às superfícies externas da placa são:

$$
\sigma_{33}= \pm q / 2 \text { para } x_{3}= \pm h / 2
$$

onde " $\mathrm{q}$ " é o carregamento transversal aplicado à placa.

\section{3 - RELAÇÕES BÁSICAS DA TEORIA DE REISSNER}

A partir das simplificações envolvidas nas hipóteses básicas serão determinadas as relações e equações que formam a teoria de Reissner. Será definido conforme a figura 2.1.a um sistema de coordenadas $x_{1} x_{2} x_{3}$, com o plano $x_{1}-x_{2}$ contendo o plano médio da placa. Na figura 2.1.b definese a convenção dos sentidos das rotações, $\phi_{1}$ no plano $x_{1}-x_{3}$ e $\phi_{2}$ no plano $x_{2}-x_{3}$.

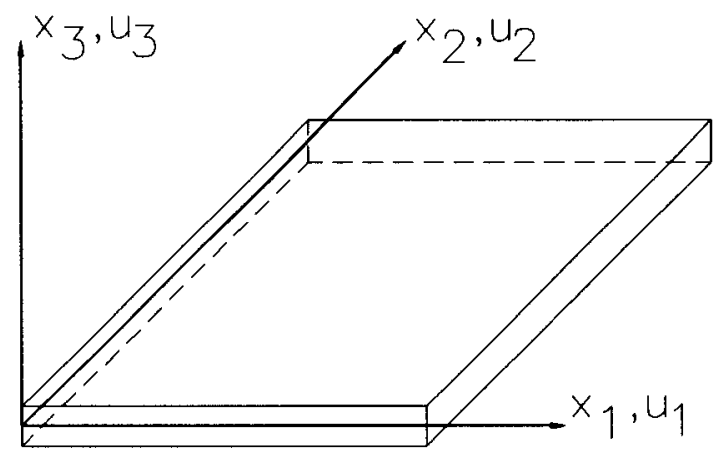

o)

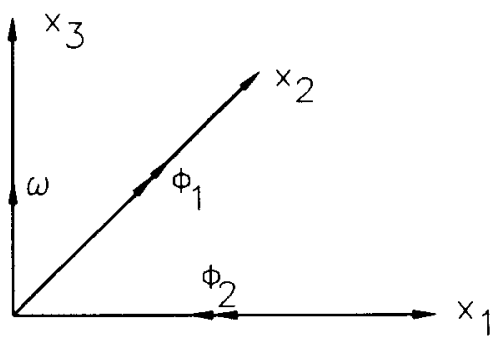

b)

Fig. 2.1 - a ) sistema de coordenadas

b ) sentido das rotações 
De acordo com as duas últimas hipóteses pode-se escrever para as superfícies limites da placa:

$$
\begin{array}{ll}
\sigma_{3 \alpha}=0 & p / x_{3}= \pm h / 2 \\
\sigma_{33}= \pm q / 2 &
\end{array}
$$

As componentes de tensão que aparecem no estudo das placas estão mostradas na figura 2.2, conforme as hipóteses básicas.

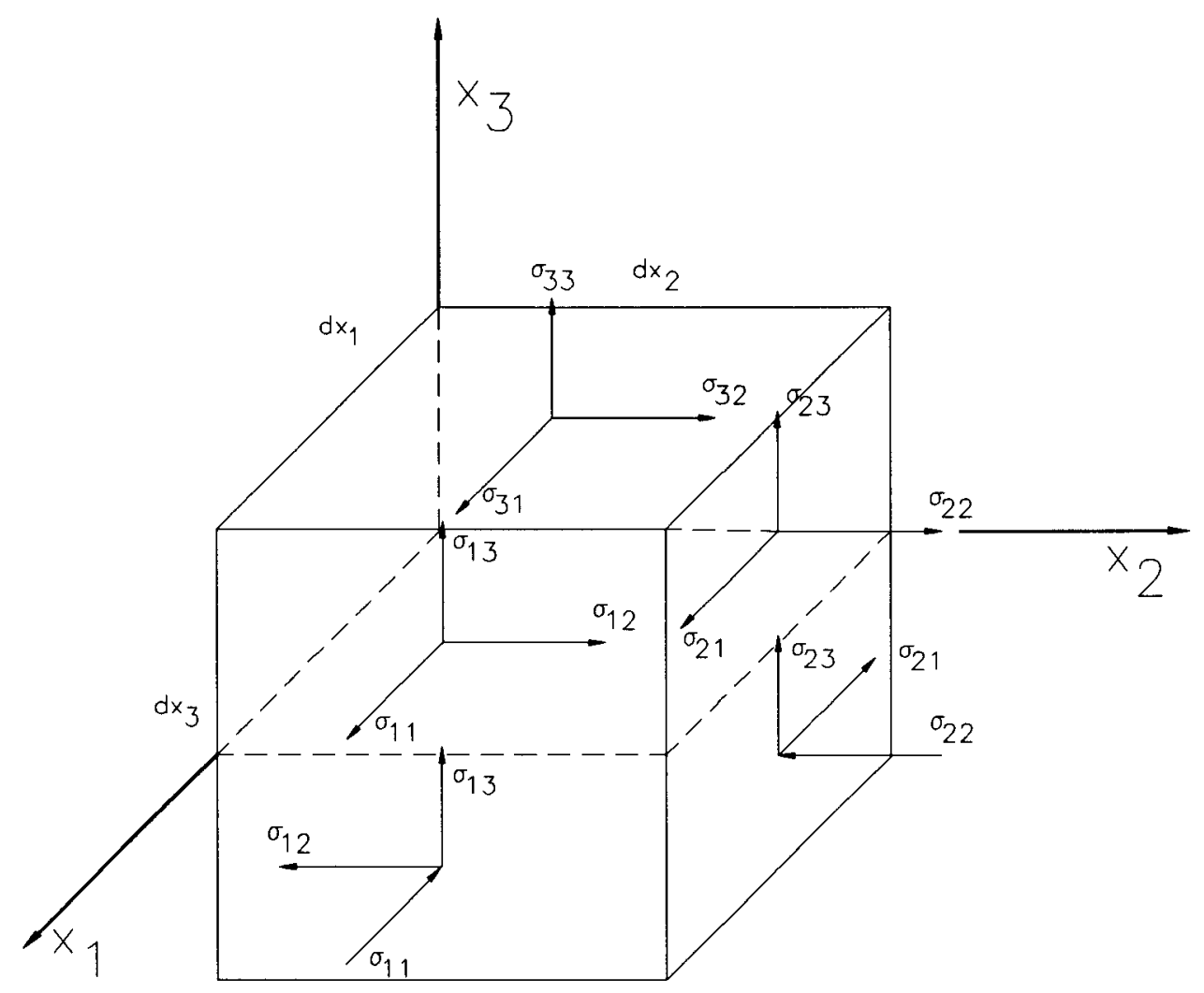

Fig. 2.2 - Componentes de tensão no elemento genérico das placas.

As resultantes de tensão são obtidas integrando-se as componentes de tensão multiplicadas por $x_{3}$, ao longo da espessura da placa. Assim, integrando-se $\sigma_{\alpha \beta}$ obtém-se momentos fletores e de torção por unidade de comprimento, dados por: 


$$
M_{\alpha \beta}=\int_{-h / 2}^{h / 2} \sigma_{\alpha \beta} x_{3} d x_{3}
$$

Analogamente integrando-se as tensões $\sigma_{\alpha 3}$ ao longo de " $\mathrm{h}$ " obtémse os esforços cortantes por unidade de comprimento:

$$
Q_{3 \alpha}=\int_{-h / 2}^{h / 2} \sigma_{\alpha 3} d x_{3}
$$

Nas expressōes (2.2) os indices em letras gregas podem assumir os valores 1 ou 2.

De acordo com a primeira e quarta hipóteses básicas, as tensões $\sigma_{\alpha \beta}$ variam linearmente na espessura e podem ser escritas como:

$$
\sigma_{\alpha \beta}=\frac{12}{h^{3}} M_{\alpha \beta} x_{3}
$$

A figura 2.3 mostra um elemento de placa onde as resultantes de tensões estão indicadas com a convenção de sinais adotada.

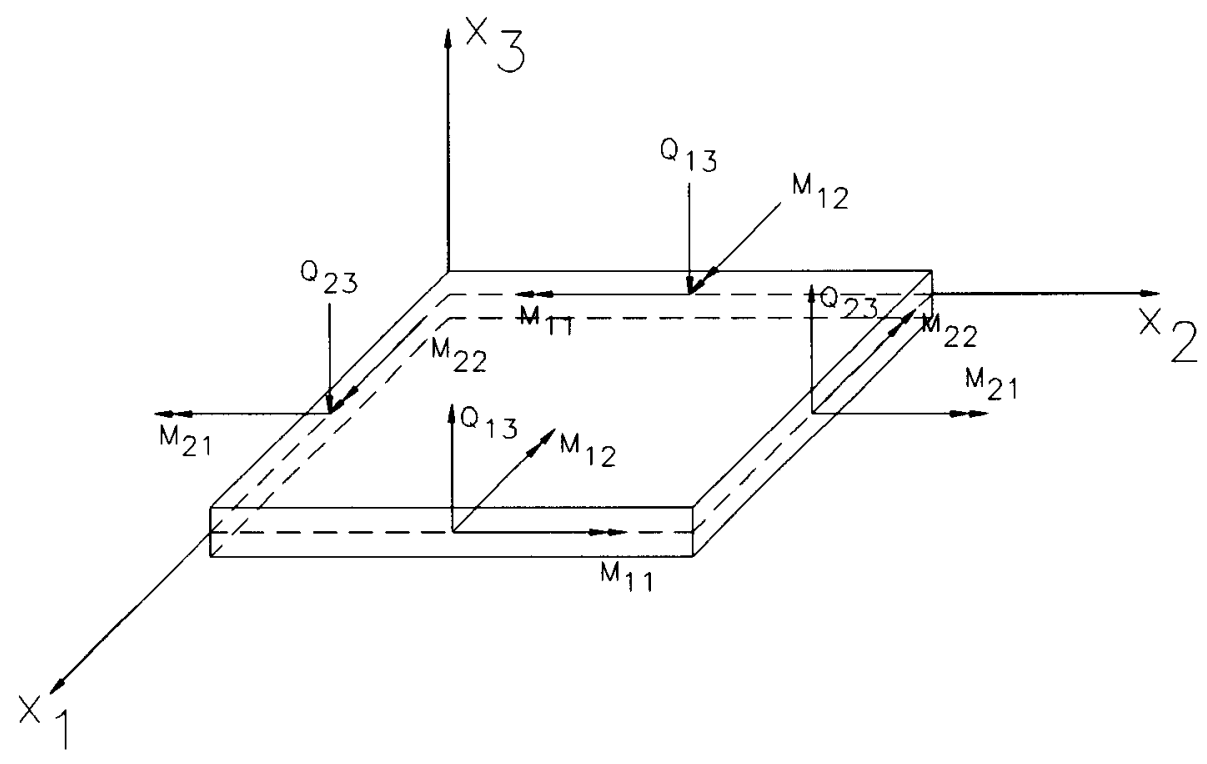

Fig. 2.3 - Momentos e cortantes resultantes. 
Segundo RIBEIRO [158], "na teoria da Elasticidade para pequenos deslocamentos, as deformações tem efeito negligenciável sobre as condições de equilíbrio, as equações de equilíbrio são deduzidas supondose a configuração indeformada da placa".

Assim, escrevendo-se o equilíbrio de forças na direção $x_{3}$ e 0 equilibrio de momentos em relação aos eixos $x_{\alpha}$, para um elemento de placa infinitesimal, obtém-se [144] :

$$
\begin{aligned}
& Q_{\alpha, \alpha}+q=0 \\
& M_{\alpha \beta, \beta}-Q_{\alpha}=0
\end{aligned}
$$

Substituindo-se as equaçōes (2.3) na equação generalizada de equilibrio das tensões dada por:

$$
\sigma_{i, j}+b_{i}=0
$$

obtém-se desprezando-se as forças de volume $b_{i}$ e levando-se em conta as equações (2.4.b):

$$
\left(\frac{12}{h^{3}}\right) Q_{\alpha} x_{3}+\partial \sigma_{\alpha 3} / \partial x_{3}=0
$$

integrando-se (2.6) em $x_{3}$ obtém-se:

$$
\sigma_{\alpha 3}=\frac{3 Q_{\alpha}}{2 h}\left[1-\left(\frac{2 x_{3}}{h}\right)^{2}\right]
$$

Levando-se as equações (2.7.a) na terceira equação de (2.5) e considerando-se (2.4.a) obtém-se: 


$$
\sigma_{33}=\frac{q x_{3}}{2 h}\left[3-\left(\frac{2 x_{3}}{h}\right)^{2}\right]
$$

Para um material elástico linear, isotrópico, o funcional da energia de deformação é o mesmo da energia de deformação complementar, que pode ser expresso em função das tensões por:

$$
U=U^{*}
$$

$$
U^{*}=\frac{1}{2 E} \int_{V}\left[\sigma_{11}^{2}+\sigma_{22}^{2}+\sigma_{33}^{2}-2 v\left(\sigma_{11} \sigma_{22}+\sigma_{11} \sigma_{33}+\sigma_{22} \sigma_{33}\right)+2(1+v)\left(\sigma_{12}^{2}+\sigma_{13}^{2}+\sigma_{23}^{2}\right)\right] d V
$$

Substituindo-se as tensões $\sigma_{\alpha \beta}$ e $\sigma_{\alpha 3}$ por suas expressões (2.3) e (2.7.a) respectivamente, e integrando-se na espessura tem-se:

$$
\begin{aligned}
& U=U^{*}=\frac{1}{2} \int_{\Omega} \frac{12}{E h^{3}}\left[\left(M_{11}+M_{22}\right)^{2}+2(1+v)\left(M_{12}^{2}-M_{11} M_{22}\right)+\right. \\
& \left.\frac{(1+v)}{5} h^{2}\left(Q_{1}^{2}+Q_{2}^{2}\right)-\frac{h^{5}}{5} q\left(M_{11}+M_{22}\right)+\frac{1}{2 E} \int_{V} \sigma_{33}^{2} d z\right] d \Omega
\end{aligned}
$$

Para a determinação das relaçōes esforço-deslocamento será usado - princípio de Hellinger-Reissner conforme RIBEIRO,J.R.M. [158] e RIBEIRO, G.O. [140], cujo funcional na ausência de forças de volume é dado por:

$$
\Pi_{R}=-U^{*}-\int_{V} \sigma_{i j, j} u_{i} d V+\int_{S_{\sigma}}\left(p_{i}-\bar{p}_{i}\right) u_{i} d S+\int_{S_{u}} p_{i} \bar{u}_{i} d S
$$

onde:

$$
\begin{aligned}
& u_{i}: \text { são deslocamentos } \\
& \bar{u}_{i}: \text { são deslocamentos prescritos no contorno }
\end{aligned}
$$


$\mathrm{p}_{\mathrm{i}}$ : são forças de superfície no contorno

$\overline{\mathrm{p}}_{i}$ : são forças prescritas no contorno

$S_{\sigma}$ : é o trecho do contorno com tensões prescritas

$S_{u}$ : é o trecho do contorno com deslocamentos prescritos

$U^{*}$ : é a energia de deformação complementar

$\sigma_{i, j}$ : é a derivada parcial da tensão $\sigma_{i j}$ na direção da coordenada $x_{i}$

No funcional ( 2.10 ) as tensōes " $\sigma_{i j}$ ", os deslocamentos " $u_{i}$ " e as forças de superfície " $p_{i}$ " atuantes em " $S_{u}$ ", são as grandezas que poderão sofrer variações.

Substituindo-se as equações (2.3) e (2.7) em (2.10) e lembrando-se que as forças " $\mathrm{p}_{\mathrm{i}}$, podem ser dadas por:

$$
p_{i}=\sigma_{i j} n_{j}
$$

onde " $n_{j}$ " são os cossenos diretores da normal ao contorno, direcionados para fora do domínio, pode-se reescrever o funcional (2.10) como:

$$
\begin{aligned}
& \Pi_{R}=-U^{*}-\int_{V}\left[\left(M_{\alpha \beta, \beta}-Q_{\alpha}\right) \frac{12}{h^{3}} u_{\alpha} x_{3}+\left(Q_{\alpha, \alpha}+q\right) \frac{3}{2 h} u_{3}\left(1-\frac{4 x_{3}}{h^{2}}\right)\right] d V+ \\
& \int_{S_{\sigma}}\left[\left(M_{\alpha \beta} n_{\beta}-\bar{M}_{\alpha \beta} n_{\beta}\right) \frac{12}{h^{3}} u_{\alpha} x_{3}+\left(Q_{\alpha} n_{\alpha}-\bar{Q}_{\alpha} n_{\alpha}\right) \frac{3}{2 h}\left(1-\frac{4 x_{3}}{h^{2}}\right)\right] d S+ \\
& \int_{S_{u}}\left[\left(M_{\alpha \beta} n_{\alpha}\right) \frac{12}{h^{3}} \bar{u}_{\alpha} x_{3}+\left(Q_{\alpha} n_{\alpha}\right) \frac{3}{2 h} \bar{u}_{3}\left(1-\frac{4 x_{3}}{h^{2}}\right)\right] d S
\end{aligned}
$$


Definem-se os deslocamentos generalizados " $w$ " e " $\phi_{\alpha}$ " como sendo respectivamente 0 deslocamento transversal médio tomado sobre a espessura da placa, e os valores médios das rotações da seção com " $x_{\alpha}$ " constante. Estes valores são obtidos igualando-se o trabalho realizado pelas tensões $e$ os deslocamentos, com 0 trabalho realizado pelas respectivas resultantes de tensão com os deslocamentos generalizados. Desta forma pode-se escrever:

$$
\begin{aligned}
& \int_{-h / 2}^{h / 2} \sigma_{11} u_{1} d x_{3}=M_{11} \phi_{1} \\
& \int_{-h / 2}^{h / 2} \sigma_{22} u_{2} d x_{3}=M_{22} \phi_{2} \\
& \int_{-h / 2}^{h / 2} \sigma_{21} u_{1} d x_{3}=M_{21} \phi_{1} \\
& \int_{-h / 2}^{h / 2} \sigma_{13} u_{3} d x_{3}=Q_{1} w \\
& \int_{-h / 2}^{h / 2} \sigma_{23} u_{3} d x_{3}=Q_{2} w
\end{aligned}
$$

Substituindo-se as equações (2.3) e (2.7.a) em (2.13) pode-se escrever genericamente:

$$
\begin{aligned}
& \phi_{\alpha}=\frac{12}{h^{3}} \int_{-h / 2}^{h / 2} u_{\alpha} x_{3} d x_{3} \\
& w=\frac{3}{2 h} \int_{-h / 2}^{h / 2} u_{3}\left[1-\left(\frac{2 x_{3}}{h}\right)^{2}\right] d x_{3}
\end{aligned}
$$


Levando-se os valores dos deslocamentos generalizados (2.14) na expressão do funcional (2.12) e fazendo-se:

$$
\begin{aligned}
& \mathrm{dV}=\mathrm{dx} x_{3} \mathrm{~d} \Omega \text { e } \mathrm{dS}=\mathrm{dx} \mathrm{x}_{3} \mathrm{~d} \Gamma, \text { obtém-se: } \\
& \Pi_{R}=-U^{*}-\int_{\Omega}\left[\left(M_{\alpha \beta, \beta}-Q_{\alpha}\right) \phi_{\alpha}+\left(Q_{\alpha, \alpha}+q\right) w\right] d \Omega+ \\
& \int_{\Gamma_{\sigma}}\left[\left(M_{\alpha \beta} n_{\beta}-\bar{M}_{\alpha \beta} n_{\beta}\right) \phi_{\alpha}+\left(Q_{\alpha} n_{\alpha}-\bar{Q}_{\alpha} n_{\alpha}\right) w\right] \alpha \Gamma_{\sigma}+ \\
& \int_{\Gamma_{u}}\left[\left(M_{\alpha \beta} n_{\alpha} \bar{\phi}_{\alpha}+Q_{\alpha} n_{\alpha} \bar{w}\right) d \Gamma_{u}\right]
\end{aligned}
$$

$\mathrm{Na}$ expressão (2.15) as grandezas sujeitas a variação são os esforços " $M_{\alpha \beta}$ ", " $Q_{\alpha}$ ", os deslocamentos generalizados " $\phi_{\alpha}$ " e " $w$ ", e as forças de contorno no trecho " $\Gamma_{u}$ " de deslocamentos prescritos.

Tomando-se a primeira variação do funcional " $\Pi_{R}$ ", integrando-se por partes e reagrupando-se os termos obtém-se segundo RIBEIRO, J.R.M [158] e RIBEIRO, G.O. [140]:

$$
\begin{aligned}
& \delta \Pi_{R}=-\int_{\Omega}\left\{\left\{\frac{12}{E h^{3}}\left[\left(M_{11}+M_{22}\right)-(1+v) M_{22}-\frac{w h^{2}}{10} q\right]-\phi_{1,1}\right\} \delta M_{11}+\right. \\
& \left\{\frac{12}{E h^{3}}\left[\left(M_{22}+M_{11}\right)-(1+v) M_{11}-\frac{w^{2}}{10} q\right]-\phi_{2,2}\right\} \delta M_{22}+ \\
& \left\{\frac{12}{E h^{3}}\left[2(1+v) M_{12}\right]-\phi_{1,2}-\phi_{2,1}\right\} \delta M_{12}+\left\{\frac{12}{E h^{3}}\left[\frac{(1+v) h^{2}}{5} Q_{1}\right]-\phi_{1}-w_{, 1}\right\} \delta Q_{1}+ \\
& \left\{\frac{12}{E h^{3}}\left[\frac{(1+v) h^{2}}{5} Q_{2}\right]-\phi_{2}-w_{, 2}\right\} \delta Q_{2}+ \\
& \left(M_{11,1}+M_{12,2}-Q_{1}\right) \delta \phi_{1}+\left(M_{21,1}+M_{22,2}-Q_{2}\right) \delta \phi_{2}+ \\
& \left.\left(Q_{1,1}+Q_{2,2}+q\right) \delta w\right\} d \Omega+\int_{\Gamma_{\sigma}}\left[\left(M_{11} n_{1}+M_{12} n_{2}-\bar{M}_{11} n_{1}-\bar{M}_{12} n_{2}\right) \delta \phi_{1}+\right.
\end{aligned}
$$




$$
\begin{aligned}
& \left.\left(M_{21} n_{1}+M_{22} n_{2}-\bar{M}_{21} n_{1}-\bar{M}_{22} n_{2}\right) \delta \phi_{2}+\left(Q_{1} n_{1}+Q_{2} n_{2}-\bar{Q}_{1} n_{1}-\bar{Q}_{2} n_{2}\right) \delta w\right] d \Gamma_{\sigma}+ \\
& \int_{\Gamma_{u}}\left[\left(\bar{\phi}_{1} n_{1}-\phi_{1} n_{1}\right) \delta M_{11}+\left(\bar{\phi}_{1} n_{2}+\bar{\phi}_{2} n_{1}-\phi_{1} n_{2}-\phi_{2} n_{1}\right) \delta M_{12}+\right. \\
& \left.\left(\bar{\phi}_{2} n_{2}-\phi_{2} n_{2}\right) \delta M_{22}+\left(\bar{w} n_{1}-w n_{1}\right) \delta Q_{1}+\left(\bar{w} n_{2}-w n_{2}\right) \delta Q_{2}\right] d \Gamma_{u}=0
\end{aligned}
$$

Para que a expressão (2.16) seja nula, todos os coeficientes das variações " $\delta M_{\alpha \beta}{ }^{\prime}$, " $\delta Q_{\alpha}$ ", " $\delta \phi_{\alpha}$ " e " $\delta W$ " devem ser simultaneamente zero, uma vez que as variações são quaisquer, mas não simultaneamente nulas. Desta forma podem ser determinadas as relações e equações básicas para a Teoria de Reissner:

a) condições de equilíbrio

$$
\begin{aligned}
& M_{11,1}+M_{12,2}-Q_{1}=0 \\
& M_{21,1}+M_{22,2}-Q_{2}=0 \\
& Q_{1,1}+Q_{2,2}-q=0
\end{aligned}
$$

b ) relações entre esforços e deslocamentos

$$
\begin{aligned}
& \frac{12}{E h^{3}}\left[M_{11}+M_{22}-(1+v) M_{22}-\frac{v h^{2}}{10} q\right]-\phi_{1,1}=0 \\
& \frac{12}{E h^{3}}\left[M_{11}+M_{22}-(1+v) M_{11}-\frac{h^{2}}{10} q\right]-\phi_{2,2}=0 \\
& \frac{24(1+v)}{E h^{3}} M_{12}-\phi_{1,2}-\phi_{2,1}=0
\end{aligned}
$$




$$
\begin{aligned}
& \frac{12(1+v)}{E h^{3}} \frac{h^{2}}{5} Q_{1}-\phi_{1}-w_{, 1}=0 \\
& \frac{12(1+v)}{E h^{3}} \frac{h^{2}}{5} Q_{2}-\phi_{2}-w_{, 2}=0
\end{aligned}
$$

c) condiçōes de contorno sobre $\Gamma_{u}$

$$
\begin{aligned}
& \phi_{1}=\bar{\phi}_{1} \\
& \phi_{2}=\bar{\phi}_{2} \\
& w=\bar{w}
\end{aligned}
$$

d ) condições de contorno sobre $\Gamma_{\sigma}$

$$
\begin{aligned}
& M_{11} n_{1}+M_{12} n_{2}=\bar{M}_{11} n_{1}+\bar{M}_{12} n_{2} \\
& M_{22} n_{1}+M_{22} n_{2}=\bar{M}_{21} n_{1}+\bar{M}_{22} n_{2} \\
& Q_{1} n_{1}+Q_{2} n_{2}=\bar{Q}_{1} n_{1}+\bar{Q}_{2} n_{2}
\end{aligned}
$$

ou genericamente

$$
\begin{aligned}
& \bar{p}_{\alpha}=\bar{M}_{\alpha \beta} n_{\beta} \\
& \bar{p}_{3}=\bar{Q}_{\alpha} n_{\alpha}
\end{aligned}
$$


A partir das equações (2.18) consegue-se explicitar os esforços " $M_{\alpha \beta}$ " e " $Q_{\alpha}$ " em função dos deslocamentos generalizados " $W$ " e " $\phi_{\alpha}$ ". Assim:

$$
\begin{aligned}
& M_{11}=D\left(\phi_{1,1}+v \phi_{2,2}\right)+\frac{v}{(1-v) \lambda^{2}} q \\
& M_{22}=D\left(\phi_{2,2}+v \phi_{1,1}\right)+\frac{v}{(1-v) \lambda^{2}} q \\
& M_{12}=D \frac{(1-v)}{2}\left(\phi_{1,2}+\phi_{2,1}\right) \\
& Q_{1}=D \frac{(1-v)}{2} \lambda^{2}\left(\phi_{1}+w_{, 1}\right) \\
& Q_{2}=D \frac{(1-v)}{2} \lambda^{2}\left(\phi_{2}+w_{, 2}\right)
\end{aligned}
$$

Escrevendo-se as expressões (2.21) de forma generalizada, obtémse:

$$
\begin{aligned}
& M_{\alpha \beta}=D \frac{(1-v)}{2}\left(\phi_{\alpha, \beta}+\phi_{\beta, \alpha}+\frac{2 v}{1-v} \phi_{y, \gamma} \delta_{\alpha \beta}\right)+\frac{v q}{(1-v) \lambda^{2}} \delta_{\alpha \beta} \\
& Q_{\alpha}=D \frac{(1-v)}{2} \lambda^{2}\left(\phi_{\alpha}+w_{, \alpha}\right)
\end{aligned}
$$

onde $D=\frac{E h^{3}}{12\left(1-v^{2}\right)}$ é a rigidez da placa à flexão e $\lambda=\frac{\sqrt{10}}{h}$ é uma constante característica das equações de Reissner.

Finalmente pode-se escrever as equaçōes dos esforços (2.22) como: 


$$
\begin{aligned}
& M_{\alpha \beta}=C_{\alpha \beta \beta \theta} \chi_{r \theta}+\frac{v q}{(1-v) \lambda^{2}} \delta_{\alpha \beta} \\
& Q_{\alpha}=C_{3 \alpha 3 \theta} \psi_{3 \theta}
\end{aligned}
$$

onde

$$
\begin{aligned}
& \chi_{r \theta}=\phi_{\gamma, \theta} \\
& \psi_{3 \theta}=\phi_{\theta}+w_{, \theta}
\end{aligned}
$$

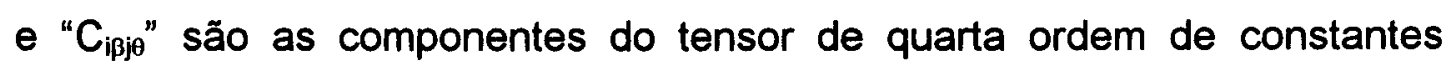
elásticas, para o caso isotrópico.

As três equações de equilíbrio (2.17) juntamente com as cinco equaçōes (2.21) formam um sistema com oito equaçōes diferenciais parciais de $1^{\text {a }}$ ordem [158], onde as oito incógnitas são os esforços " $M_{11}$ ", " $M_{12}$ ", " $M_{22}$ ", " $Q_{1}$ " e " $Q_{2}$ " e os deslocamentos " $w$ ", " $\phi_{1}$ " e " $\phi_{2}$ ". Este sistema forma a base da teoria de Reissner para flexão de placas com pequenos deslocamentos e conduz naturalmente a três condições físicas em cada ponto do contorno.

O sistema anterior pode ser condensado em um outro de três equaçōes diferenciais parciais, aumentando-se consequentemente a ordem do sistema de tal forma a manter as mesmas três condições físicas em cada ponto do contorno. Isto é feito explicitando-se " $\phi_{1}$ " e " $\phi_{2}{ }^{\prime}$ em (2.21.b) e substituindo-se estes valores nas expressões (2.21.a), obtendo-se:

$$
\begin{aligned}
& M_{11}=-D\left(w_{, 11}+v w_{.22}\right)+\frac{2}{(1-v) \lambda^{2}}\left(Q_{1,1}+v Q_{2,2}\right)+\frac{v}{(1-v) \lambda^{2}} q \\
& M_{22}=-D\left(w_{, 22}+v w_{, 11}\right)+\frac{2}{(1-v) \lambda^{2}}\left(Q_{2,2}+v Q_{1,1}\right)+\frac{v}{(1-v) \lambda^{2}} q \\
& M_{12}=-D(1-v) w_{, 12}+\frac{1}{\lambda^{2}}\left(Q_{1,2}+Q_{2,1}\right)
\end{aligned}
$$


Utilizando-se a terceira equação de equilíbrio (2.17) nas equações (2.24) obtém-se:

$$
\begin{aligned}
& M_{11}=-D\left(w_{, 11}+v w_{, 22}\right)+\frac{2}{\lambda^{2}} Q_{1,1}-\frac{v}{(1-v) \lambda^{2}} q \\
& M_{22}=-D\left(w_{, 22}+v w_{, 11}\right)+\frac{2}{\lambda^{2}} Q_{2,2}-\frac{v}{(1-v) \lambda^{2}} q \\
& M_{12}=-D(1-v) w_{, 12}+\frac{1}{\lambda^{2}}\left(Q_{1,2}+Q_{2,1}\right)
\end{aligned}
$$

Substituindo-se as equaçōes (2.25) nas duas primeiras equaçōes de (2.17) obtém-se:

$$
\begin{aligned}
& Q_{1}-\frac{1}{\lambda^{2}} \nabla^{2} Q_{1}+\frac{1}{(1-v) \lambda^{2}} \frac{\partial q}{\partial x_{1}}=-D \frac{\partial}{\partial x_{1}} \nabla^{2} w \\
& Q_{2}-\frac{1}{\lambda^{2}} \nabla^{2} Q_{2}+\frac{1}{(1-v) \lambda^{2}} \frac{\partial q}{\partial x_{2}}=-D \frac{\partial}{\partial x_{2}} \nabla^{2} w
\end{aligned}
$$

Derivando-se a primeira equação de (2.26) em relação a $x_{1}$ e a segunda em relação a $x_{2}$, e levando-se na terceira equação de equilibrio (2.17), obtém-se:

$$
D \nabla^{4} w=q-\frac{(2-v)}{(1-v)} \frac{1}{\lambda^{2}} \nabla^{2} q
$$

O novo sistema de equações diferenciais é formado pelas equações (2.26) e (2.27). 
Admitindo-se a possibilidade da ocorrência de momentos distribuídos " $m_{\alpha}$ " na superfície média da placa, as equaçōes de equilíbrio (2.17) devem ser modificadas para contemplar esta nova situaçăo, e indicialmente podem ser escritas como:

$$
\begin{aligned}
& M_{\alpha \beta, \beta}-Q_{\alpha}+m_{\alpha}=0 \\
& Q_{\alpha, \alpha}+q=0
\end{aligned}
$$

\section{4 - CONDIÇÖES DE CONTORNO}

No item anterior mostrou-se que para satisfazer o sistema de equaçōes diferenciais envolvido na teoria de Reissner é necessário atender a três condiçōes físicas para cada ponto do contorno.

Em placas retangulares as condições de contorno usuais são: borda simplesmente apoiada, borda engastada e borda livre.

$\mathrm{Na}$ borda simplesmente apoiada pode-se prescrever duas formas distintas de condiçōes de contorno:

$$
w=0, M_{n}=0 \text { e } \phi_{s}=0 \quad \text { ("hard condition") }
$$

ou

$$
w=0, M_{n}=0 \text { e } M_{n s}=0 \quad \text { ("soft condition") }
$$

onde " $n$ " e "s" são os eixos normal e tangencial ao contorno, respectivamente. 
A primeira condição prescreve a rotaçăo tangencial nula na borda, que é o normalmente usado na teoria clássica de Kirchhoff. A segunda já prescreve o momento de torção " $M_{n s}$ " nulo no contorno, sendo considerada exata por RIBEIRO [158].

Para borda engastada tem-se:

$$
w=0 \quad \phi_{n}=0 \quad \phi_{s}=0
$$

Para borda livre tem-se:

$$
M_{n}=0 \quad M_{n s}=0 \quad Q_{n}=0
$$

\section{5 - SOLUÇÃO FUNDAMENTAL DE PLACAS USANDO A TEORIA DE REISSNER}

Como este trabalho tem por base o uso de equações integrais de contorno obtidas através da teoria de Reissner para flexão de placas, tornase necessário para o equacionamento das mesmas, a formulação das soluçōes fundamentais das placas pela referida teoria. Entende-se por solução fundamental a resposta em um ponto genérico " $x$ " (ponto campo) de um domínio, em geral considerado infinito $\left(\Omega^{*}\right)$, devido à aplicação de uma carga unitária em outro ponto " $\xi$ " (ponto fonte) deste mesmo domínio, cujo contorno é dado por $\Gamma^{*}$.

Exprimindo-se as equações de equilibrio em termos dos deslocamentos generalizados, obtém-se: 


$$
\Delta_{i i}^{*}\left(\frac{\partial}{\partial \xi}\right) u_{j}(\xi)=-b_{i}(\xi)
$$

onde $\Delta_{i j}^{*}$ representa as componentes do operador de Navier dadas por:

$$
\begin{aligned}
& \Delta_{\alpha \beta}^{*}=\frac{D(1-v)}{2}\left[\left(\nabla^{2}-\lambda^{2}\right) \delta_{\alpha \beta}+\frac{1+v}{1-v} \frac{\partial^{2}}{\partial x_{\alpha}(\xi) \partial x_{\beta}(\xi)}\right] \\
& \Delta_{\alpha 3}^{*}=-\Delta_{3 \alpha}^{*}=-\frac{D(1-v)}{2} \lambda^{2} \frac{\partial}{\partial x_{\alpha}(\xi)} \\
& \Delta_{33}^{*}=\frac{D(1-v)}{2} \lambda^{2} \nabla^{2}
\end{aligned}
$$

onde $\nabla^{2}=\frac{\partial^{2}}{\partial x_{\alpha} \partial x_{\alpha}}$ é o operador de Laplace.

Admitindo-se que o carregamento unitário referido na definição da solução fundamental, seja representado numericamente pela função Delta de Dirac " $\delta(\xi, x)$ ", com singularidade no ponto fonte " $\xi$ ", tem-se:

$$
\Delta_{i j}^{*}\left(\frac{\partial}{\partial x}\right) u_{k j}^{*}(\xi, x)=-\delta(\xi, x) \delta_{i k}
$$

Na expressão (2.31) " $\delta_{\mathrm{ik}}$ " representa $\circ$ Delta de Kronecker e " $u_{k j}^{*}(\xi, x)$ " a solução fundamental em termos de deslocamentos.

De acordo com WEEËN [143], a solução fundamental é expressa por: 


$$
\begin{aligned}
& u_{\alpha \beta}^{*}=\frac{1}{8 \pi D(1-v)}\left\{[8 B-(1-v)(2 \ln z-1)] \delta_{\alpha \beta}-[8 A+2(1-v)] r_{, \alpha} r_{, \beta}\right\} \\
& u_{\alpha 3}^{*}=-u_{3 \alpha}^{*}=\frac{1}{8 \pi D}(2 \ln z-1) r r_{, \alpha} \\
& u_{33}^{*}=\frac{1}{8 \pi D(1-v) \lambda^{2}}\left[(1-v) z^{2}(\ln z-1)-8 \ln z\right]
\end{aligned}
$$

onde " $r$ " é a distância entre o ponto fonte " $\xi$ " e o ponto campo " $x$ ". As componentes de " $r$ " e as derivadas na direção " $\alpha$ " são dadas por:

$$
\begin{aligned}
& r_{\alpha}=x_{\alpha}(x)-x_{\alpha}(\xi) \\
& r_{, \alpha}=\frac{\partial r}{\partial x_{\alpha}(x)}=\frac{r_{\alpha}}{r} \\
& z=\lambda r
\end{aligned}
$$

Os valores de "A" e " $B$ " que aparecem em (2.32) são função de " $Z$ " dadas por:

$$
\begin{aligned}
& A(z)=k_{0}(z)+\frac{2}{z}\left[k_{1}(z)-\frac{1}{z}\right] \\
& B(z)=k_{0}(z)+\frac{1}{z}\left[k_{1}(z)-\frac{1}{z}\right]
\end{aligned}
$$

sendo " $k_{0}(z)$ " e " $k_{1}(z)$ " funções de Bessel modificadas de ordem inteira, dadas em ABRAMOWITZ \& STEGUN [112]. Mostra-se que a expressão de " $A(z)$ " é contínua e que " $B(z)$ " apresenta singularidade do tipo "In $z$ " (ver apêndice A). 


\section{ELEMENTOS DE CONTORNO APLICADOS À \\ ANÁLISE DE PLACAS PELA TEORIA DE REISSNER}

\section{1- INTRODUÇÃO}

Neste capitulo serão desenvolvidas as equações integrais de placas, utilizando-se a teoria de Reissner, apresentada no capítulo anterior, visando a formulação do problema de flexão de placas através do Método dos Elementos de Contorno.

Utilizando-se o método dos resíduos ponderados serão deduzidas inicialmente equações integrais para deslocamentos em pontos do domínio e do contorno da placa. Posteriormente estas representações integrais dos deslocamentos serão transformadas, com base nas relações esforçosdeslocamentos em equações integrais para os esforços em pontos do domínio. Será considerada a possibilidade de atuação de cargas transversais distribuidas em áreas ou linhas, cargas concentradas além de momentos " $m_{\alpha}$ " distribuídos em linha.

A análise de flexão de placas através do Método dos Elementos de Contorno conduz a um sistema de equações integrais, cuja solução analítica é descartada em função da solução numérica, que transforma-o em um 
sistema de equações algébricas lineares. Esta transformação implica em uma discretizaçăo do contorno em segmentos, denominados elementos de contorno, sobre os quais serão aproximados com funções previamente escolhidas, os deslocamentos e as forças de superfície. Analogamente, as integrais de domínio que aparecem nas equações integrais serão transformadas em integrais sobre o contorno da região carregada.

Em cada ponto de colocação serão geradas três equações correspondentes às rotações nas direções normal e tangencial ao contorno e uma de deslocamento transversal " $W$ ".

Impondo-se as condições de contorno, o sistema é resolvido e em seguida calculam-se de forma direta os deslocamentos e esforços para pontos internos.

3.2 - EQUAÇŐES INTEGRAIS DE PLACAS PELA TEORIA DE REISSNER

\subsection{1 -EQUAÇÕES INTEGRAIS PARA DESLOCAMENTOS EM PONTOS DO DOMINIO}

A partir das equações básicas da teoria de Reissner apresentadas no capítulo anterior, serão deduzidas neste item as equações integrais para deslocamentos em pontos do domínio.

Considera-se a placa da Fig. 3.1, de espessura constante " $h$ ", em equilíbrio sob a ação de carregamentos transversais distribuídos " $q$ ", cargas 
concentradas verticais " $P_{i}$ " e momentos externos distribuidos " $m_{\alpha}$ ", todos aplicados no domínio.

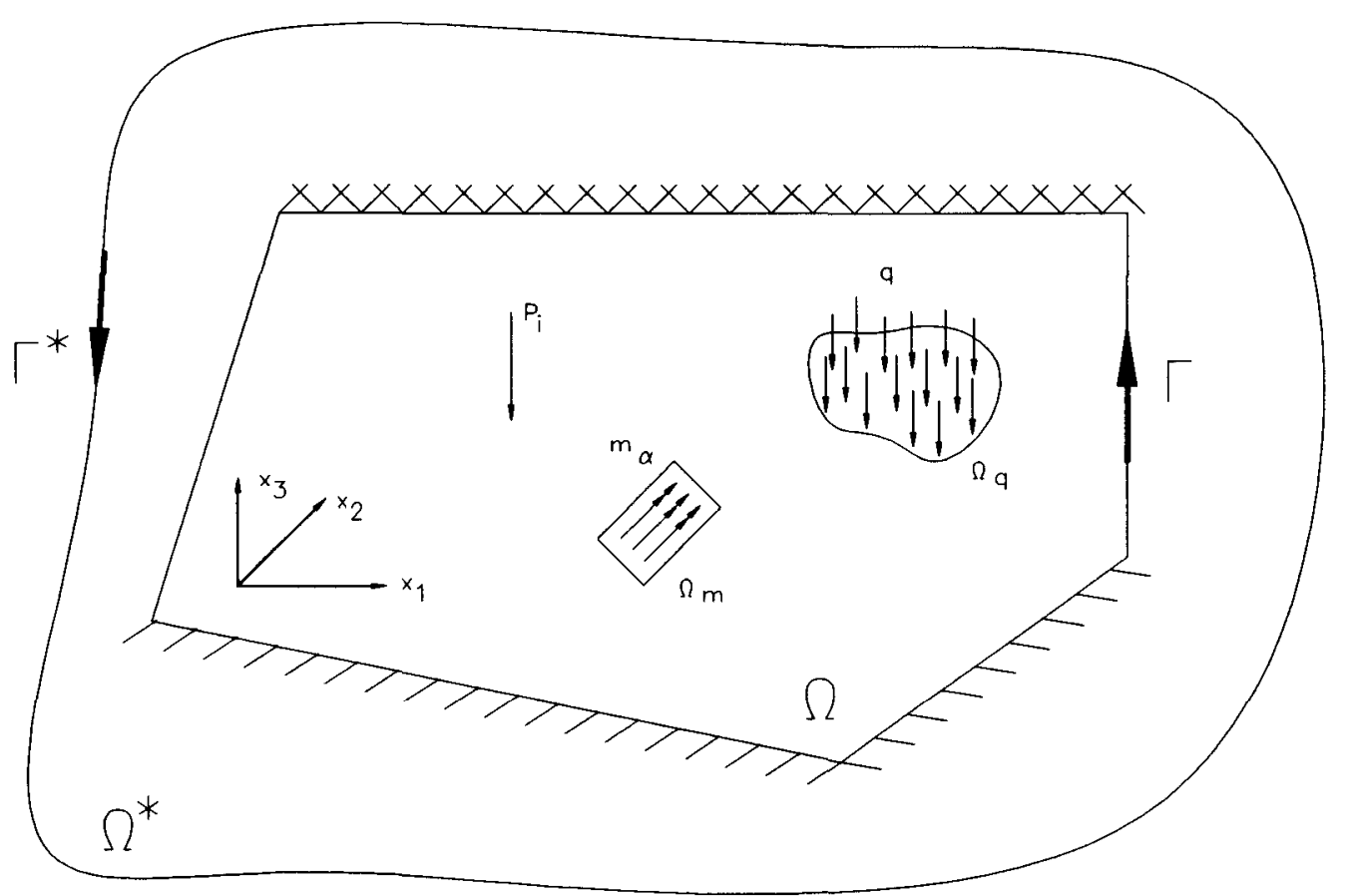

Fig. 3.1 - Placa finita de espessura constante.

A equação integral para deslocamentos em pontos internos pode ser obtida pelo método dos resíduos ponderados, utilizando-se a solução fundamental como função ponderadora, a fim de se obter uma função aproximada para as equações de equilíbrio (2.28) [144]. O erro cometido nesta aproximação pode ser distribuído de acordo com [140]:

$$
\int_{\Omega}\left(\sigma_{i j, j}+b_{i}\right) u_{i}^{*} d \Omega=\int_{\Gamma_{p}}\left(p_{i}-\bar{p}_{i}\right) u_{i}^{*} d \Gamma_{p}-\int_{\Gamma_{u}}\left(u_{i}-\bar{u}_{i}\right) p_{i}^{*} d \Gamma_{u}
$$

onde " $\Gamma_{p}$ " $e$ " $\Gamma_{u}$ " são trechos do contorno onde " $p_{i}$ " $e$ " $u_{i}$ são prescritos respectivamente.

Aplicando-se a equação (3.1) à situação da placa, submetida às condiçōes de equilíbrio dadas por (2.17) obtém-se: 
41

$$
\begin{aligned}
& \int_{\Omega}\left[\left(M_{\alpha \beta, \beta}-Q_{\alpha}+m_{\alpha}\right) u_{\alpha}^{*}+\left(Q_{\alpha, \alpha}+q\right) u_{3}^{*}\right] d \Omega= \\
& \int_{\Gamma_{p}}\left(p_{i}-\bar{p}_{i}\right) u_{i}^{*} d \Gamma_{p}+\int_{\Gamma_{u}}\left(u_{i}-\bar{u}_{i}\right) p_{i}^{*} d \Gamma_{u}
\end{aligned}
$$

Nas expressões (3.1) e (3.2) " $u_{i}^{*}$ " é a solução fundamental em termos de deslocamentos, que neste caso já representa deslocamentos generalizados. A solução fundamental em termos de forças de superfície " $p_{i}^{*}$, que será analisada posteriormente, representa aqui também forças fundamentais generalizadas.

Integrando-se por partes o primeiro e quarto termo do primeiro membro da equação (3.2), tem-se:

$$
\begin{aligned}
& \int_{\Gamma} M_{\alpha \beta} n_{\beta} u_{\alpha}^{*} d \Gamma-\int_{\Omega} M_{\alpha \beta} u_{\alpha, \beta}^{*} d \Omega+\int_{\Omega}\left(-Q_{\alpha}+m_{\alpha}\right) u_{\alpha}^{*} d \Omega+ \\
& \int_{\Gamma} Q_{\alpha} n_{\alpha} u_{3}^{*} d \Gamma-\int_{\Omega} Q_{\alpha} u_{3, \alpha}^{*} d \Omega+\int_{\Omega_{q}} q u_{3}^{*} d \Omega_{q}= \\
& \int_{\Gamma_{p}}\left(p_{i}-\bar{p}_{i}\right) u_{i}^{*} d \Gamma_{p}+\int_{\Gamma_{u}}\left(u_{i}-\bar{u}_{i}^{*}\right) p_{i}^{*} d \Gamma_{u}
\end{aligned}
$$

onde " $n_{\alpha}$ " representa a componente da normal ao contorno referido ao eixo $" x_{\alpha} "$.

De acordo com (2.11) pode-se escrever:

$$
\int_{\Gamma} M_{\alpha \beta} n_{\beta} u_{\alpha}^{*} d \Gamma=\int_{\Gamma} p_{\alpha} u_{\alpha}^{*} d \Gamma
$$


42

$$
\int_{\Gamma} Q_{\alpha} n_{\alpha} u_{3}^{*} d \Gamma=\int_{\Gamma} p_{3} u_{3}^{*} d \Gamma
$$

e que

$$
\int_{\Gamma} p_{\alpha} u_{\alpha}^{*} d \Gamma+\int_{\Gamma} p_{3} u_{3}^{*} d \Gamma=\int_{\Gamma} p_{i} u_{i}^{*} d \Gamma
$$

A equação (3.3) pode ser reescrita como:

$$
\begin{aligned}
& -\int_{\Omega} M_{\alpha \beta} u_{\alpha, \beta}^{*} d \Omega-\int_{\Omega} Q_{\alpha}\left(u_{\alpha}^{*}+u_{3, \alpha}^{*}\right) d \Omega+\int_{\Omega}\left(m_{\alpha} u_{\alpha}^{*}+q u_{3}^{*}\right) d \Omega= \\
& -\int_{\Gamma_{u}} p_{i} u_{i}^{*} d \Gamma_{u}-\int_{\Gamma_{p}} \bar{p}_{i} u_{i}^{*} d \Gamma_{p}-\int_{\Gamma_{u}}\left(u_{i}-\bar{u}_{i}\right) p_{i}^{*} d \Gamma_{u}
\end{aligned}
$$

Substituindo-se as equações (2.23) em (3.4) obtém-se:

$$
-\int_{\Omega} C_{\alpha \beta \gamma \theta} u_{\alpha, \beta}^{*} \phi_{\gamma, \theta} d \Omega-\int_{\Omega} \frac{v q}{(1-v) \lambda^{2}} \delta_{\alpha \beta} u_{\alpha, \beta}^{*} d \Omega-\int_{\Omega} C_{3 \alpha 3 \theta}\left(u_{\alpha}^{*}+u_{3, \alpha}^{*}\right)\left(\phi_{\theta}+w_{, \theta}\right) d \Omega+
$$

$$
\int_{\Omega}\left(m_{\alpha} u_{\alpha}^{*}+q u_{3}^{*}\right) d \Omega=-\int_{\Gamma_{u}} p_{i} u_{i}^{*} d \Gamma_{u}-\int_{\Gamma_{p}} \bar{p}_{i} u_{i}^{*} d \Gamma_{p}-\int_{\Gamma_{u}}\left(u_{i}-\bar{u}_{i}\right) p_{i}^{*} d \Gamma_{u}
$$

Lembrando-se que:

$$
\phi_{\gamma, \theta}=u_{\gamma, \theta}
$$

$$
u_{\alpha}^{*}+u_{3, \alpha}^{*}=\psi_{3 \alpha}^{*}
$$

$$
\phi_{\theta}+w_{, \theta}=\psi_{3 \theta}
$$

e fazendo-se: 
43

$$
\begin{aligned}
& C_{\alpha \beta \gamma \theta} u_{\alpha, \beta}^{*}=M_{\gamma \theta}^{*} \\
& C_{3 \alpha 3 \theta} \psi_{3 \alpha}^{*}=Q_{\theta}^{*}
\end{aligned}
$$

a expressão (3.5) transforma-se em:

$$
\begin{aligned}
& -\int_{\Omega} M_{\gamma \theta}^{*} u_{\gamma, \theta} d \Omega-\frac{v}{(1-v) \lambda^{2}} \int_{\Omega} q u_{\alpha, \alpha}^{*} d \Omega-\int_{\Omega} Q_{\theta}^{*} \psi_{3 \theta} d \Omega+\int_{\Omega}\left(m_{\alpha} u_{\alpha}^{*}+q u_{3}^{*}\right) d \Omega= \\
& -\int_{\Gamma_{u}} p_{i} u_{i}^{*} d \Gamma_{u}-\int_{\Gamma_{p}} \bar{p}_{i} u_{i}^{*} d \Gamma_{p}-\int_{\Gamma_{u}}\left(u_{i}-\bar{u}_{i}\right) p_{i}^{*} d \Gamma_{u}
\end{aligned}
$$

Considerando-se a equação (2.23.c) e integrando-se por partes o primeiro e terceiro termos de (3.6) obtém-se:

$$
\begin{aligned}
& -\int_{\Gamma} \mathrm{M}_{\gamma \theta}^{*} \mathrm{n}_{\theta} \mathrm{u}_{\gamma} \mathrm{d} \Gamma+\int_{\Omega} \mathrm{M}_{\gamma \theta \theta}^{*} \mathrm{u}_{\gamma} \mathrm{d} \Omega-\frac{v}{(1-v) \lambda^{2}} \int_{\Omega} q \mathrm{u}_{\alpha, \alpha}^{*} \mathrm{~d} \Omega-\int_{\Omega} \mathrm{Q}_{\theta}^{*} \mathrm{u}_{\theta} \mathrm{d} \Omega- \\
& \int_{\Gamma} Q_{\theta}^{*} n_{\theta} u_{3} d \Gamma+\int_{\Omega} Q_{\theta, \theta}^{*} u_{3} d \Omega+\int_{\Omega}\left(m_{\alpha} u_{\alpha}^{*}+q u_{3}^{*}\right) d \Omega= \\
& -\int_{\Gamma_{u}} p_{i} u_{i}^{*} d \Gamma_{u}-\int_{\Gamma_{p}} \bar{p}_{i} u_{i}^{*} d \Gamma_{p}-\int_{\Gamma_{u}}\left(u_{i}-\bar{u}_{i}\right) p_{i}^{*} d \Gamma_{u}
\end{aligned}
$$

observando-se que:

$$
\begin{aligned}
& \int_{\Gamma} M_{\gamma \theta}^{*} n_{\theta} u_{\gamma} d \Gamma+\int_{\Gamma} Q_{\theta}^{*} n_{\theta} u_{3} d \Gamma=\int_{\Gamma} u_{i} p_{i}^{*} d \Gamma \\
& \int_{\Gamma} u_{i} p_{i}^{*} d \Gamma-\int_{\Gamma_{u}} u_{i} p_{i}^{*} d \Gamma_{u}=\int_{\Gamma p} u_{i} p_{i}^{*} d \Gamma_{p}
\end{aligned}
$$

e reagrupando-se os termos do primeiro membro de (3.7) obtém-se: 


$$
\begin{aligned}
& \int_{\Omega} u_{r}\left(M_{\gamma \theta, \theta}^{*}-Q_{r}^{*}\right) d \Omega+\int_{\Omega} u_{3} Q_{\theta, \theta}^{*} d \Omega+\int_{\Omega} q\left[u_{3}^{*}-\frac{v}{(1-v) \lambda^{2}} u_{\alpha, \alpha}^{*}\right] d \Omega+ \\
& \int_{\Omega} m_{\alpha} u_{\alpha}^{*} d \Omega=-\int_{\Gamma_{u}} p_{i} u_{i}^{*} d \Gamma_{u}-\int_{\Gamma_{p}} \bar{p}_{i} u_{i}^{*} d \Gamma_{p}+\int_{\Gamma_{p}} u_{i} p_{i}^{*} d \Gamma_{p}+\int_{\Gamma_{u}} \bar{u}_{i} p_{i}^{*} d \Gamma_{u}
\end{aligned}
$$

Considerando-se que:

$$
\begin{aligned}
& \int_{\Gamma_{p}} u_{i} p_{i}^{*} d \Gamma_{p}+\int_{\Gamma_{u}} \bar{u}_{i} p_{i}^{*} d \Gamma_{u}=\int_{\Gamma} u_{i} p_{i}^{*} d \Gamma \\
& \int_{\Gamma_{u}} p_{i} u_{i}^{*} d \Gamma_{u}+\int_{\Gamma_{p}} \bar{p}_{i} u_{i}^{*} d \Gamma_{p}=\int_{\Gamma} p_{i} u_{i}^{*} d \Gamma
\end{aligned}
$$

e lembrando-se que as soluções fundamentais originaram-se da aplicação de um carregamento unitário, representado pelo Delta de Dirac, que tem as seguintes particularidades:

$$
\begin{aligned}
& M_{r \theta, \theta}^{*}-Q_{\gamma}^{*}+\delta(\xi, x) C_{\gamma}(\xi)=0 \\
& Q_{\gamma, y}^{*}+\delta(\xi, x) C_{3}(\xi)=0 \\
& \delta(\xi, x)=0 \quad \text { se " } x \text { " é diferente de " } \xi^{\prime \prime} \\
& \delta(\xi, x)=\infty \text { " é igual a " } \xi^{\prime \prime} \\
& \int_{\Omega^{*}} u(x) \delta(\xi, x) d \Omega^{*}(x)=u(\xi) \\
& u_{i}^{*}=u_{i j}^{*}(\xi, x) C_{j} \\
& p_{i}^{*}=p_{i j}^{*}(\xi, x) C_{j}
\end{aligned}
$$

a equação (3.8) pode finalmente ser escrita como: 


$$
\begin{aligned}
& \mathrm{u}_{\mathrm{i}}(\xi)=\int_{\Gamma}\left[\mathrm{u}_{\mathrm{ij}}^{*}(\xi, \mathrm{X}) \mathrm{p}_{\mathrm{j}}(\mathrm{X})-\mathrm{p}_{\mathrm{ij}}^{*}(\xi, \mathrm{X}) \mathrm{u}_{\mathrm{j}}(\mathrm{X})\right] \mathrm{d} \Gamma(\mathrm{X})+ \\
& \int_{\Omega_{q}} q(x)\left[u_{i 3}^{*}(\xi, x)-\frac{v}{(1-v) \lambda^{2}} u_{i \alpha, \alpha}^{*}(\xi, x)\right] d \Omega(x)+\int_{\Omega_{m}} u_{i \alpha}^{*}(\xi, x) m_{\alpha}(x) d \Omega(x)
\end{aligned}
$$

onde " $\Omega_{q}$ " e " $\Omega_{m}$ " são respectivamente os subdomínios de atuação da carga distribuida e do momento externo distribuído.

A equação (3.11) representa os deslocamentos dos pontos " $\xi$ " do domínio em função dos valores dos deslocamentos e das forças de superfície para pontos " $\mathrm{X}$ " do contorno, quando a placa está submetida aos carregamentos " $q(x)$ " e " $m_{\alpha}(x)$ " aplicados no domínio.

Os valores " $u_{i j}^{*}(\xi, x)$ " e " $p_{i j}^{*}(\xi, x)$ " em (3.11) representam respectivamente o deslocamento e a força generalizada na direção "j" em " $x$ " (ponto campo), correspondentes a uma força generalizada unitária, concentrada na direção "i" do ponto " $\xi$ " (fonte).

\subsection{2 -EQUAÇŐES INTEGRAIS PARA DESLOCAMENTOS EM PONTOS DO CONTORNO.}

A equação (3.11) foi definida para pontos " $\xi$ " do dominio, entretanto para a formulação do problema de flexão de placas pelo Método dos Elementos de Contorno é necessário a obtenção de equações integrais para pontos " $\chi$ " do contorno.

Considere-se a Fig. 3.2, onde um ponto " $\chi$ " inicialmente no contorno, torna-se pertencente ao domínio pelo acréscimo de um contorno circular " $\Gamma_{\varepsilon}$, de centro em " $\chi$ " e raio " $\varepsilon$ ". 

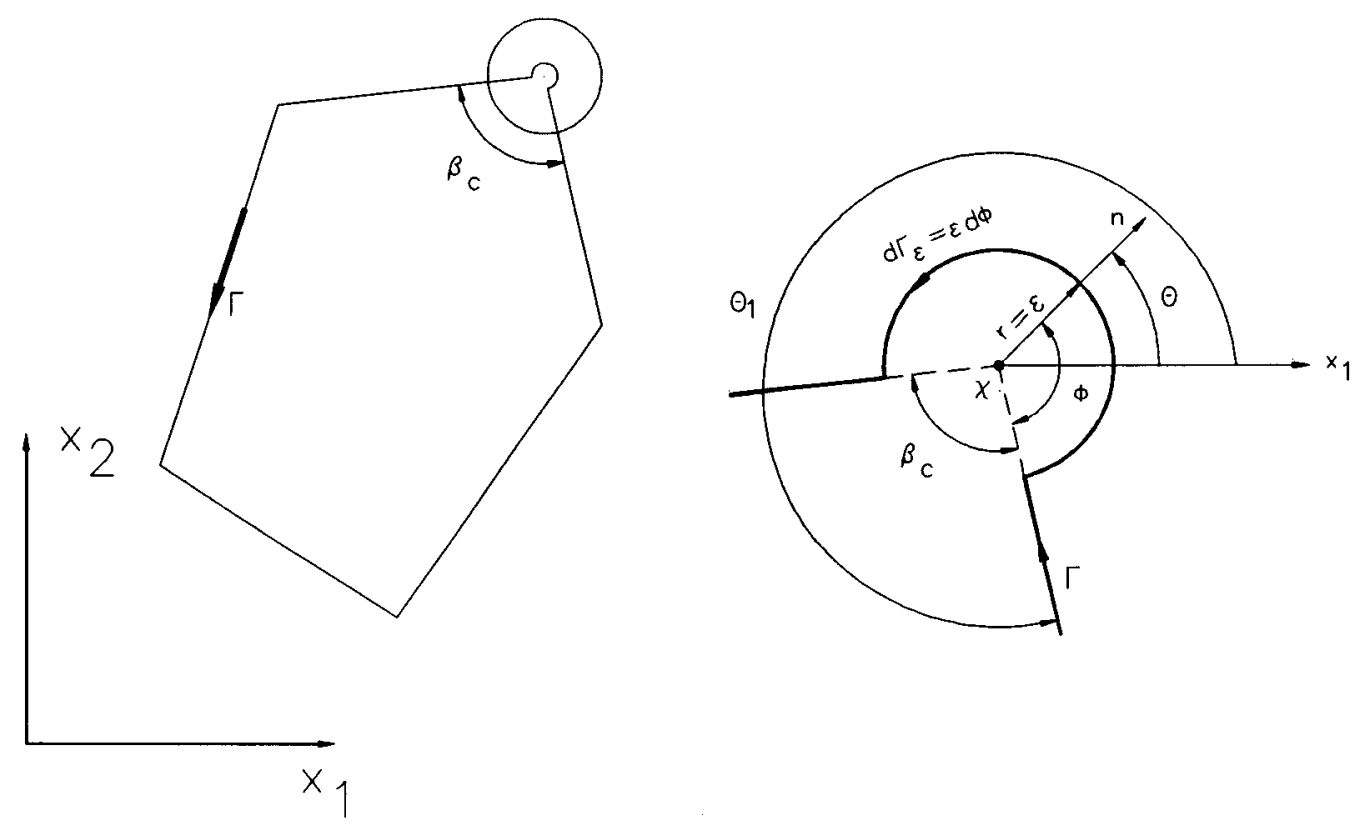

Fig. 3.2 - Placa com alteração do contorno no canto.

No contorno " $\Gamma_{\varepsilon}$ " acrescido, o ângulo " $\phi$ " varia de 0 (zero) a $\left(2 \pi-\beta_{c}\right)$ enquanto " $\theta$ " varia de " $\theta_{1}$ " a $\left(\theta_{1}-\beta_{c}\right)$.

Como o ponto " $\chi$ " com esta alteração pertence agora ao domínio pode-se aplicar a equação (3.11), onde o contorno " $\Gamma^{n}$ é acrescido de um contorno circular " $\Gamma_{\varepsilon}$ " e suprimido de uma parcela " $\bar{\Gamma}$ ".

Assim:

$$
\begin{aligned}
& u_{i}(\chi)=\int_{\Gamma-\Gamma}\left[u_{i j}^{*}(\chi, X) p_{j}(X)-p_{i j}^{*}(\chi, X) u_{j}(X)\right] \pi \Gamma(X)+ \\
& \int_{\Gamma_{e}}\left[u_{i j}^{*}(\chi, X) p_{j}(X)-p_{i j}^{*}(\chi, X) u_{j}(X)\right] \Omega \Gamma_{s}(X)+ \\
& \int_{\Omega_{q}} q(x)\left[u_{i 3}^{*}(\chi, x)-\frac{v}{(1-v) \lambda^{2}} u_{i \alpha, \alpha}^{*}(\chi, x)\right] d \Omega(x)+\int_{\Omega_{m}} u_{i \alpha}^{*}(\chi, x) m_{\alpha}(x) d \Omega(x)
\end{aligned}
$$

Quando o raio " $\varepsilon$ " do contorno " $\Gamma_{\varepsilon}$ " tender a zero, o ponto " $\chi$ " aproximará para o contorno, tendo-se na situação limite: 


$$
\begin{aligned}
& u_{i}(\chi)=\lim _{\Gamma \rightarrow 0} \int_{\Gamma-\bar{\Gamma}}\left[u_{i j}^{*}(\chi, X) p_{j}(X)-p_{i j}^{*}(\chi, X) u_{j}(X)\right] \Gamma(X)+ \\
& \lim _{s \rightarrow 0} \int_{\Gamma_{s}}\left[u_{i j}^{*}(\chi, X) p_{j}(X)-p_{i j}^{*}(\chi, X) u_{j}(X)\right] \beta \Gamma_{s}(X)+ \\
& \int_{\Omega_{q}} q(x)\left[u_{i j}^{*}(\chi, x)-\frac{v}{(1-v) \lambda^{2}} u_{i \alpha, \alpha}^{*}(\chi, x)\right] d \Omega(x)+\int_{\Omega_{m}} u_{i \alpha}^{*}(\chi, x) m_{\alpha}(x) d \Omega(x)
\end{aligned}
$$

Em (3.13) os limites sobre " $\Gamma-\bar{\Gamma}^{n}$ serão efetuados no sentido do valor principal de Cauchy, o limite sobre " $\Gamma_{\varepsilon}{ }^{n}$ do termo " $u_{i j}^{*}(\chi, x)$ " se anula, restando apenas a determinação do limite sobre " $\Gamma_{\varepsilon}$ " do termo " $p_{i j}^{*}(\chi, x)$ ". Devido a continuidade de " $u_{j}(\chi)$ " este último limite pode ser escrito como:

$$
\lim _{\varepsilon \rightarrow 0} \int_{\Gamma_{\varepsilon}} p_{i j}^{*}(\chi, X) u_{j}(X) d \Gamma_{s}(X)=\lim _{s \rightarrow 0} u_{j}(\chi) \int_{\Gamma_{s}} p_{i j}^{*}(\chi, X) d \Gamma_{\varepsilon}(X)
$$

Agrupando-se (3.14) com o primeiro termo de (3.13) obtém-se a equação integral para deslocamentos em pontos do contorno expressa por:

$$
\begin{gathered}
c_{i j}(\chi) u_{j}(\chi)=\int_{\Gamma}\left[u_{i j}^{*}(\chi, X) p_{j}(X)-p_{i j}^{*}(\chi, X) u_{j}(X)\right] \Gamma(X)+ \\
\int_{\Omega_{q}} q(x)\left[u_{i j}^{*}(\chi, x)-\frac{v}{(1-v) \lambda^{2}} u_{i \alpha, \alpha}^{*}(\chi, x)\right] d \Omega_{q}(x)+\int_{\Omega_{m}} u_{i \alpha}^{*}(\chi, x) m_{\alpha}(x) d \Omega_{m}(x)
\end{gathered}
$$

onde

$$
\begin{aligned}
& c_{i j}(\chi)=\delta_{i j}+\lim _{s \rightarrow 0} \int_{\Gamma_{s}} p_{i j}^{*}(\chi, x) d \Gamma(x) \\
& \lim _{\varepsilon \rightarrow 0} \int_{\Gamma_{c}} p_{11}^{*}(\chi, x) d \Gamma(x)=\lim _{\varepsilon \rightarrow 0} \int_{\Gamma_{s}} p_{22}^{*}(\chi, x) d \Gamma(x)= \\
& \frac{\beta_{c}}{2 \pi}-1+\frac{(1+v)}{4 \pi}\left[\operatorname{sen}^{2} \beta_{c} \operatorname{sen} 2 \theta_{1}-\frac{1}{2} \operatorname{sen} 2 \beta_{c}\left(2 \operatorname{sen}^{2} \theta_{1}-1\right)\right]
\end{aligned}
$$




$$
\begin{aligned}
& \lim _{\varepsilon \rightarrow 0} \int_{\Gamma_{c}} p_{12}^{*}(\chi, x) d \Gamma(x)=\lim _{\delta \rightarrow 0} \int_{\Gamma_{s}} p_{21}^{*}(\chi, x) d \Gamma(x)= \\
& -\frac{(1-v)}{2 \pi}\left[\left(1-\cos 2 \beta_{c}\right) \cos 2 \theta_{1}-\operatorname{sen} 2 \theta_{1} \operatorname{sen} 2 \beta_{c}\right] \\
& \lim _{\varepsilon \rightarrow 0} \int_{\Gamma_{c}} p_{\alpha 3}^{*}(\chi, x) d \Gamma(x)=\lim _{\varepsilon \rightarrow 0} \int_{\Gamma_{c}} p_{3 \alpha}^{*}(\chi, x) d \Gamma(x)=0 \\
& \lim _{\varepsilon \rightarrow 0} \int_{\Gamma_{c}} p_{33}^{*}(\chi, x) d \Gamma(x)=\frac{\beta_{c}}{2 \pi}-1
\end{aligned}
$$

\subsection{3 -FORÇAS FUNDAMENTAIS GENERALIZADAS NO CONTORNO}

Nas equações dos deslocamentos para pontos do domínio e do contorno, expressões (3.11) e (3.17) respectivamente, apareceram termos em " $p_{i j}^{*}$, que são as forças fundamentais generalizadas no contorno. Estas forças serão deduzidas neste item a partir da definição (2.11) e das soluções fundamentais obtidas em (2.32). As forças generalizadas podem ser escritas como:

$$
\begin{aligned}
& p_{\gamma \alpha}^{*}(\xi, x)=M_{\alpha \beta \gamma}^{*}(\xi, x) n_{\beta} \\
& p_{3 \alpha}^{*}(\xi, x)=M_{\alpha \beta 3}^{*}(\xi, x) n_{\beta} \\
& p_{\alpha 3}^{*}(\xi, x)=Q_{3 \beta \alpha}^{*}(\xi, x) n_{\beta} \\
& p_{33}^{*}(\xi, x)=Q_{3 \beta 3}^{*}(\xi, x) n_{\beta}
\end{aligned}
$$


sendo

$M_{\alpha \beta \gamma}^{*}$ : momentos fundamentais “ $M_{\alpha \beta}^{*}$ " em “ $x^{n}$, produzidos por momentos unitários aplicados em " $\xi$ ", nas direções " $\gamma$ ".

$M_{\alpha \beta 3}^{*}$ : momentos fundamentais " $M_{\alpha \beta}^{*}$ " em " $x^{\prime}$, produzidos por uma força unitária aplicada em " $\xi$ ", na direção " $x_{3}$ ".

$Q_{3 \beta \alpha}^{*}$ : forças cortantes fundamentais " $Q_{3 \beta}^{*}$ " em "x", produzidas por momentos unitários aplicados em " $\xi$ ", nas direções " $\alpha$ ".

$Q_{3 \beta 3}^{*}$ : forças cortantes fundamentais " $Q_{3 \beta}^{*}$ " em "x", produzidas por uma força unitária aplicada em " $\xi$ ", na direção " $x_{3}$ ".

A partir das equações (2.22) pode-se escrever:

$$
\begin{aligned}
& M_{\alpha \beta \gamma}^{*}=\frac{D(1-v)}{2}\left(u_{\gamma \beta, \alpha}^{*}+u_{\gamma \alpha, \beta}^{*}+\frac{2 v}{1-v} u_{\gamma \theta, \theta}^{*} \delta_{\alpha \beta}\right) \\
& M_{\alpha \beta 3}^{*}=\frac{D(1-v)}{2}\left(u_{3 \beta, \alpha}^{*}+u_{3 \alpha, \beta}^{*}+\frac{2 v}{1-v} u_{3 \theta, \theta}^{*} \delta_{\alpha \beta}\right) \\
& Q_{3 \beta \alpha}^{*}=\frac{D(1-v) \lambda^{2}}{2}\left(u_{\alpha \beta}^{*}+u_{\alpha 3, \beta}^{*}\right) \\
& Q_{3 \beta 3}^{*}=\frac{D(1-v) \lambda^{2}}{2}\left(u_{3 \beta}^{*}+u_{33, \beta}^{*}\right)
\end{aligned}
$$

As derivadas de " $u_{i j}^{*}$ serão obtidas a partir das derivadas das equações (2.31) em relação às coordenadas do ponto " $x$ ". Considerando-se as equações (2.32) e que as derivadas de " $A(z)$ ", $B(z)$ " e " $r_{, \alpha}$ " são dadas por:

$$
\frac{\partial z}{\partial x_{\alpha}(x)}=\lambda r_{, \alpha}
$$




$$
\begin{aligned}
& \frac{\partial A(z)}{\partial x_{\alpha}(x)}=-\frac{r_{, \alpha}}{r}\left(z k_{1}+2 A\right) \\
& \frac{\partial B(z)}{\partial x_{\alpha}(x)}=-\frac{r_{, \alpha}}{r}\left(z k_{1}+A\right) \\
& \frac{\partial r_{, \alpha}}{\partial x_{\beta}(x)}=\frac{\delta_{\alpha \beta}-r_{, \alpha} r_{, \beta}}{r}
\end{aligned}
$$

Usando-se (2.31), (2.32) e (3.20) as derivadas de " $u_{i j}^{*}$ podem ser expressas por:

$$
\begin{aligned}
& u_{\alpha \beta, \gamma}^{*}=\frac{1}{4 \pi r D(1-v)}\left[\left(4 A+4 z k_{1}+1-v\right) r_{, \gamma} \delta_{\alpha \beta}-2\left(2 z k_{1}+8 A+1-v\right) r_{, \alpha} r_{, \beta} r_{, \gamma}+\right. \\
& \left.(4 A+1-v)\left(r_{, \alpha} \delta_{\beta \gamma}+r_{, \beta} \delta_{\alpha \beta}\right)\right] \\
& u_{\alpha \beta, \beta}^{*}=-\frac{r_{, \alpha}}{2 \pi r D} \\
& u_{\alpha 3, \beta}^{*}=-u_{3 \alpha, \beta}^{*}=\frac{1}{8 \pi D}\left[\delta_{\alpha \beta}(2 \ln z-1)+2 r_{, \alpha} r_{, \beta}\right] \\
& u_{\alpha 3, \alpha}^{*}=-u_{3 \alpha, \alpha}^{*}=\frac{\ln z}{2 \pi D} \\
& u_{33, \alpha}^{*}=-\frac{r_{, \alpha}}{8 \pi r^{2} D(1-v)}\left[(1-v) z^{2}(2 \ln z-1)-8\right]
\end{aligned}
$$

Substituindo-se os valores (3.21) em (3.19) e levando-se estes novos resultados em (3.18) obtém-se as seguintes expressões para " $p_{i j}^{*}$ : 


$$
\begin{aligned}
& p_{\alpha \beta}^{*}=-\frac{1}{4 \pi r}\left[\left(4 A+2 z k_{1}+1-v\right)\left(\delta_{\alpha \beta} r_{, n}+r_{, \beta} n_{\alpha}\right)+(4 A+1+v) r_{, \alpha} n_{\beta}-\right. \\
& \left.2\left(8 A+2 z k_{1}+1-v\right) r_{, \alpha} r_{, \beta} r_{, n}\right] \\
& p_{\alpha 3}^{*}=\frac{\lambda^{2}}{2 \pi}\left(B n_{\alpha}-A r_{, \alpha} r_{, n}\right) \\
& p_{3 \alpha}^{*}=-\frac{(1-v)}{8 \pi}\left[\left(\frac{2+2 v}{1-v} \ln z-1\right) n_{\alpha}+2 r_{, \alpha} r_{, n}\right] \\
& p_{33}^{*}=-\frac{1}{2 \pi r} r_{, n}
\end{aligned}
$$

onde " $r, n$ " é a derivada de " $r$ " na direção normal no ponto " $x$ ", dada por:

$$
r_{, n}=\frac{\partial}{\partial n(x)}=\frac{\partial}{\partial x_{\alpha}(x)} \frac{\partial x_{\alpha}(x)}{\partial n}=r_{, \alpha} n_{, \alpha}
$$

Examinando-se as expressões (3.22) e as expansões de " $A(z)$ " e "B(z)" conclui-se que " $p_{i j}^{* \prime}$ possui singularidades do tipo "In r" e " $1 / r$ ".

\subsection{4 -EQUAÇÕES INTEGRAIS PARA ESFORÇOS NOS PONTOS DO DOMINIO.}

$\mathrm{Na}$ análise de placas é de grande interesse a determinação das tensões em pontos internos, e consequentemente as suas resultantes, que são os momentos fletores e de torção e as forças cortantes. No Método dos Elementos de Contorno estas grandezas são calculadas diretamente a partir 
da utilização de equações integrais próprias. Do ponto de vista de precisão e eficiência computacional este procedimento é mais apropriado, do que calcular deslocamentos em pontos internos e derivá-los numericamente para a obtenção dos esforços, como é feito no Método dos Elementos Finitos.

Os esforços " $M_{\alpha \beta}$ " e " $Q_{\alpha}$ " estão expressos genericamente em (2.22). As grandezas envolvidas nestas expressōes são os deslocamentos generalizados " $\phi_{\alpha}$ " e " $W$ " e suas derivadas. A representação integral para deslocamentos generalizados em pontos internos é dada em (3.11). Portanto para se obter a equação integral dos esforços em pontos internos basta aplicar as derivações envolvidas em (2.22) na equação integral dos deslocamentos (3.11). Deve-se ressaltar que estas derivadas são agora em relação ao ponto interno " $\xi$ " e não mais em relação ao ponto " $\mathrm{X}$ " do contorno.

Analisando-se (2.33) nota-se que:

$$
\frac{\partial r}{\partial x_{\alpha}(\xi)}=-\frac{r_{\alpha}}{r}=-r_{, \alpha}
$$

e que portanto para se ter as derivadas (3.20) em relação a " $x_{\alpha}(\xi)$ " basta multiplicá-las por (-1).

Assim:

$$
\begin{aligned}
& \frac{\partial z}{\partial x_{\alpha}(\xi)}=-\lambda r_{, \alpha} \\
& \frac{\partial A(z)}{\partial x_{\alpha}(\xi)}=\frac{r_{, \alpha}}{r}\left(z k_{1}+2 A\right)
\end{aligned}
$$




$$
\begin{aligned}
& \frac{\partial B(z)}{\partial x_{\alpha}(\xi)}=\frac{r_{, \alpha}}{r}\left(z k_{1}+A\right) \\
& \frac{\partial r_{, \alpha}}{\partial x_{\alpha}(\xi)}=\frac{r_{, \alpha} r_{, \beta}-\delta_{\alpha \beta}}{r} \\
& \frac{\partial \partial_{, n}}{\partial x_{\alpha}(\xi)}=\frac{r_{, \alpha} r_{, n}-n_{\alpha}}{r}
\end{aligned}
$$

Aplicando-se portanto (2.22) em (3.11) obtém-se os esforços " $M_{\alpha \beta}$ " e " $Q_{\alpha}$ " dados por:

$$
\begin{aligned}
& M_{\alpha \beta}(\xi)=\int_{\Gamma} u_{\alpha \beta k}^{*}(\xi, X) p_{k}(X) d \Gamma(X)-\int_{\Gamma} p_{\alpha \beta k t}^{*}(\xi, X) u_{k}(X) d \Gamma(X)+ \\
& \int_{\Omega_{q}} \mathrm{w}_{\alpha \beta}^{*}(\xi, \mathrm{x}) \mathrm{q}(\mathrm{x}) \mathrm{d} \Omega(\mathrm{x})+\int_{\Omega_{\mathrm{m}}} \mathrm{u}_{\alpha \beta \gamma}^{*}(\xi, \mathrm{x}) \mathrm{m}_{\gamma}(\mathrm{x}) \mathrm{d} \Omega(\mathrm{x})+\frac{v}{(1-v) \lambda^{2}} \mathrm{q}(\xi) \delta_{\alpha \beta} \\
& Q_{3 \beta}(\xi)=\int_{\Gamma} u_{3 \beta k}^{*}(\xi, X) p_{k}(X) d \Gamma(X)-\int_{\Gamma} p_{3 \beta k}^{*}(\xi, X) u_{k}(X) d \Gamma(X)+ \\
& \int_{\Omega_{q}} w_{3 \beta}^{*}(\xi, x) q(x) d \Omega(x)+\int_{\Omega_{m}} u_{3 \beta \gamma}^{*}(\xi, x) m_{\gamma}(x) d \Omega(x)
\end{aligned}
$$

onde:

$$
\begin{aligned}
& u_{\alpha \beta \gamma}^{*}=\frac{D(1-v)}{2}\left(u_{\alpha \gamma, \beta}^{*}+u_{\beta \gamma, \alpha}^{*}+\frac{2 v}{1-v} u_{\theta \gamma, \theta}^{*} \delta_{\alpha \beta}\right) \\
& u_{\alpha \beta 3}^{*}=\frac{D(1-v)}{2}\left(u_{\alpha 3, \beta}^{*}+u_{\beta 3, \alpha}^{*}+\frac{2 v}{1-v} u_{3 \theta, \theta}^{*} \delta_{\alpha \beta}\right) \\
& u_{3 \beta \gamma}^{*}=\frac{D(1-v) \lambda^{2}}{2}\left(u_{\beta \gamma}^{*}+u_{3 \gamma, \beta}^{*}\right)
\end{aligned}
$$




$$
\begin{aligned}
& u_{3 \beta 3}^{*}=\frac{D(1-v) \lambda^{2}}{2}\left(u_{\beta 3}^{*}+u_{33, \beta}^{*}\right) \\
& p_{\alpha \beta \gamma}^{*}=\frac{D(1-v)}{2}\left(p_{\alpha \gamma, \beta}^{*}+p_{\beta \gamma, \alpha}^{*}+\frac{2 v}{1-v} p_{\theta \gamma, \theta}^{*} \delta_{\alpha \beta}\right) \\
& p_{\alpha \beta 3}^{*}=\frac{D(1-v)}{2}\left(p_{\alpha 3, \beta}^{*}+p_{\beta 3, \alpha}^{*}+\frac{2 v}{1-v} p_{\theta 3, \theta}^{*} \delta_{\alpha \beta}\right) \\
& p_{3 \beta \gamma}^{*}=\frac{D(1-v) \lambda^{2}}{2}\left(p_{\beta \gamma}^{*}+p_{3 \gamma, \beta}^{*}\right) \\
& p_{3 \beta 3}^{*}=\frac{D(1-v) \lambda^{2}}{2}\left(p_{\beta 3}^{*}+p_{33, \beta}^{*}\right) \\
& w_{\alpha \beta}^{*}=\frac{D(1-v)}{2}\left[u_{\alpha 3, \beta}^{*}+u_{\beta 3, \alpha}^{*}+\frac{2 v}{1-v} u_{\theta 3, \theta}^{*} \delta_{\alpha \beta}-\right. \\
& \left.\frac{v}{(1-v) \lambda^{2}}\left(u_{\alpha \gamma, \gamma \beta}^{*}+u_{\beta \gamma, \gamma \alpha}^{*}+\frac{2 v}{1-v} u_{\theta \gamma, \gamma \theta}^{*} \delta_{\alpha \beta}\right)\right] \\
& w_{3 \beta}^{*}=\frac{D(1-v) \lambda^{2}}{2}\left[u_{\beta 3}^{*}+u_{33, \beta}^{*}-\frac{v}{(1-v) \lambda^{2}}\left(u_{\beta \alpha, \alpha}^{*}+u_{3 \alpha, \alpha \beta}^{*}\right)\right]
\end{aligned}
$$

Considerando-se (3.25) derivam-se as soluções fundamentais " $u_{i j}^{*}$ ", que substituídas em (3.28.a) fornecem:

$$
\begin{aligned}
& u_{\alpha \beta \gamma}^{*}=\frac{1}{4 \pi r}\left[\left(4 A+2 z k_{1}+1-v\right)\left(r_{, \alpha} \delta_{\beta \gamma}+r_{, \beta} \delta_{\alpha \gamma}\right)-2\left(2 z k_{1}+8 A+1-v\right) r_{, \alpha} r_{, \beta} r_{, \gamma}+\right. \\
& \left.(4 A+1+v) r_{, \gamma} \delta_{\alpha \beta}\right] \\
& u_{\alpha \beta 3}^{*}=-\frac{(1-v)}{8 \pi}\left\{\left[\frac{2(1+v)}{(1-v)} \ln z-1\right] \delta_{\alpha \beta}+2 r_{, \alpha} r_{, \beta}\right\}
\end{aligned}
$$




$$
\begin{aligned}
& u_{3 \beta \gamma}^{*}=\frac{\lambda^{2}}{2 \pi}\left(\mathrm{B}_{\beta \gamma}-\mathrm{Ar}_{, \beta} \mathrm{r}_{, y}\right) \\
& u_{3 \beta 3}^{*}=\frac{r_{, \beta}}{2 \pi r}
\end{aligned}
$$

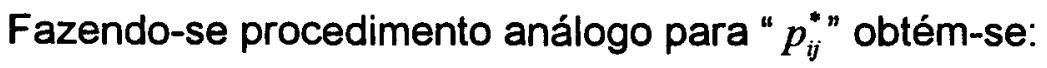

$$
\begin{aligned}
& p_{\alpha \beta \gamma}^{*}=\frac{D(1-v)}{4 \pi r^{2}}\left\{\left(4 A+2 z k_{1}+1-v\right)\left(n_{\alpha} \delta_{\beta \gamma}+n_{\beta} \delta_{\alpha \gamma}\right)+(4 A+1+3 v) n_{\gamma} \delta_{\alpha \beta}-\right. \\
& \left(16 A+6 z k_{1}+z^{2} k_{0}+2-2 v\right)\left(r_{, \gamma}\left(n_{\alpha} r_{, \beta}+n_{\beta} r_{, \alpha}\right)+r_{, n}\left(r_{, \alpha} \delta_{\beta \gamma}+r_{, \beta} \delta_{\alpha \gamma}\right)\right]- \\
& 2\left(8 A+2 z k_{1}+1+v\right)\left(r_{, a} r_{, \beta} n_{\gamma}+r_{r} r_{, n} \delta_{\alpha \beta}\right)+ \\
& \left.4\left(24 A+8 z k_{1}+z^{2} k_{0}+2-2 v\right) r_{, \alpha} r_{, \beta} r_{, \gamma} r_{, n}\right\} \\
& p_{\alpha \beta 3}^{*}=\frac{D(1-v) \lambda^{2}}{4 \pi r}\left[\left(2 A+z k_{1}\right)\left(n_{\alpha} r_{, \beta}+n_{\beta} r_{, \alpha}\right)-2\left(4 A+z k_{1}\right) r_{, \alpha} r_{, \beta} r_{, n}+2 A r_{, n} \delta_{\alpha \beta}\right] \\
& p_{3 \beta \gamma}^{*}=\frac{-D(1-v) \lambda^{2}}{4 \pi r}\left[\left(2 A+z k_{1}\right)\left(n_{\beta} r_{, \gamma}+r_{, n} \delta_{\gamma \beta}\right)+2 A r_{, \beta} n_{\gamma}-2\left(4 A+z k_{1}\right) r_{r, \gamma} r_{, \beta} r_{, n}\right] \\
& p_{3 \beta 3}^{*}=\frac{D(1-v) \lambda^{2}}{4 \pi r^{2}}\left[\left(B z^{2}+1\right) n_{\beta}-\left(A z^{2}+2\right) r_{, \beta} r_{, n}\right]
\end{aligned}
$$

A influência da carga distribuída " $q(x)^{n}$ nos esforços internos é dada por " $w_{\alpha \beta}^{*}$ " $e$ " $w_{3 \beta}^{*}$ ", que depois de efetuadas as derivadas e substituídas em (3.28.c) fornecem: 


$$
w_{\alpha \beta}^{*}=\frac{-1-v}{8 \pi}\left\{\delta_{\alpha \beta}\left[\frac{2(1+v)}{1-v} \ln z-1\right]+2 r_{, \alpha} r_{, \beta}-\frac{4 v}{(1-v) \lambda^{2} r^{2}}\left(2 r_{, \alpha} r_{, \beta}-\delta_{\alpha \beta}\right)\right\}
$$

$$
w_{3 \beta}^{*}=\frac{r_{, \beta}}{2 \pi r}
$$

Conforme (3.26) e (3.27) a influência dos momentos distribuídos " $m_{\alpha}(x)$ ", aplicados à placa em " $\Omega_{m}(x)^{n}$, nos esforços internos é dada por " $u_{\alpha \beta \gamma}^{*}$ " e " $u_{3 \beta r}^{*}$ ", valores já determinados em (3.28.a).

\subsection{5 - CARGAS E MOMENTOS DISTRIBUÍDOS NO DOMINIO}

Observando-se tanto as equações integrais de deslocamentos (3.11) e (3.15), como as equações integrais para esforços (3.26) e (3.27), nota-se que os efeitos da carga distribuída " $q(x)$ " e dos momentos distribuídos " $m_{\alpha}$ ", na obtenção destes valores, são calculados através de integrais sobre os domínios internos " $\Omega_{q}$ " $\mathrm{e}$ " $\Omega_{\mathrm{m}}$ " respectivamente.

A carga " $q(x)$ " pode assumir neste trabalho dois tipos de distribuição: uniforme e linear. Em ambos os casos a integral sobre o domínio " $\Omega_{q}$ " será transformada numa integral sobre o contorno da região carregada " $\Gamma_{q}$ ". Conforme WEEËN $[43,143]$, para carga " $q(x)$ " uniformemente distribuida, a integral sobre o domínio " $\Omega_{q}$ " pode ser transformada em:

$$
\int_{\Omega_{q}} q(x)\left[u_{i 3}^{*}(\xi, x)-\frac{v}{(1-v) \lambda^{2}} u_{i \alpha, \alpha}^{*}(\xi, x)\right] d \Omega_{q}(x)=
$$




$$
q(x) \int_{\Gamma_{q}}\left[V_{i, \alpha}^{*}(\xi, x)-\frac{v}{(1-v) \lambda^{2}} u_{i \alpha}^{*}(\xi, x)\right] n_{\alpha} d \Gamma_{q}(x)
$$

onde a função " $V_{i, \alpha}^{*}(\xi, x)$ " deve satisfazer a:

$$
\mathrm{V}_{i, \alpha \alpha}^{*}(\xi, x)=u_{i 3}^{*}(\xi, x)
$$

As expressões fundamentais " $\mathrm{V}_{i}^{*}(\xi, x)$ " que atendem a condição (3.33) são dadas por:

$$
\begin{aligned}
& \mathrm{V}_{\alpha}^{*}=\frac{1}{128 \pi D \lambda^{2}} r_{, \alpha} r z^{2}(4 \ln z-5) \\
& \mathrm{V}_{3}^{*}=\frac{-z^{2}}{256 \pi D \lambda^{4}(1-v)}\left[64(\ln z-1)-z^{2}(1-v)(2 \ln z-3)\right]
\end{aligned}
$$

e suas derivadas são dadas por:

$$
\begin{aligned}
& \mathrm{V}_{\alpha, \beta}^{*}=\frac{r^{2}}{128 \pi D}\left[\delta_{\alpha \beta}(4 \ln z-5)+2(4 \ln z-3) r_{, \alpha} r_{, \beta}\right] \\
& \mathrm{V}_{3, \beta}^{*}=\frac{-r r_{, \beta}}{128 \pi D(1-v) \lambda^{2}}\left[32(2 \ln z-1)-z^{2}(1-v)(4 \ln z-5)\right]
\end{aligned}
$$

A equação 3.32 representa a influência da carga " $q(x)$ " constante, atuando no subdomínio " $\Omega_{q}$ ", no cálculo dos deslocamentos.

A influência da carga distribuída no cálculo dos esforços internos que aparece em (3.26) e (3.27) integrada em relação ao domínio " $\Omega_{\mathrm{q}}$ ", será agora transformada em integral sobre o contorno " $\Gamma_{q}$ ", conforme (3.32), que 
depois de efetuadas as derivadas envolvidas nas equações generalizadas para esforços internos (2.21), fornece:

$$
\begin{array}{ll}
q(x) \int_{\Gamma_{q}} W_{\alpha \beta}^{*}(\xi, x) d \Gamma_{q}(x) & \text { (para momentos) } \\
q(x) \int_{\Gamma_{q}} W_{3 \beta}^{*}(\xi, x) d \Gamma_{q}(x) & \text { (para forças cortantes) }
\end{array}
$$

Em (3.35) " $q(x)$ " é constante e os valores " $W_{i \beta}^{*}$ " se referem à integração sobre o contorno " $\Gamma_{q}$ ", sendo dadas por:

$$
\begin{aligned}
& W_{\alpha \beta}^{*}=\frac{D(1-v)}{2}\left[V_{\alpha, \gamma \beta}^{*}+V_{\beta, \gamma \alpha}^{*}+\frac{2 v}{1-v} V_{\theta \gamma, \theta}^{*} \delta_{\alpha \beta}\right]-\frac{v}{(1-v) \lambda^{2}} u_{\alpha \beta \gamma}^{*} n_{\gamma} \\
& W_{3 \beta}^{*}=\frac{D(1-v) \lambda^{2}}{2}\left(V_{\beta, \gamma}^{*}+V_{3, \gamma \beta}^{*}\right) n_{\gamma}-\frac{v}{(1-v) \lambda^{2}} u_{3 \beta \gamma}^{*} n_{\gamma}
\end{aligned}
$$

onde os valores " $u_{\alpha \beta \gamma}^{*}$ " e " $u_{3 \beta \gamma}^{*}$ " são dados em (3.28.a).

Derivando-se as expressões (3.34.b) em relação ao ponto " $\xi$ ", conforme (3.36) obtém-se:

$$
\begin{aligned}
& W_{\alpha \beta}^{*}=-\frac{r}{64 \pi}\left\{(4 \ln z-3)\left[(1-v)\left(r_{, \alpha} n_{\beta}+r_{, \beta} n_{\alpha}\right)+(1+3 v) r_{, n} \delta_{\alpha \beta}\right]+\right. \\
& \left.4\left[(1-v) r_{, \alpha} r_{, \beta}+v \delta_{\alpha \beta}\right] r_{, n}\right\}-\frac{v}{(1-v) \lambda^{2}} u_{\alpha \beta \gamma}^{*} n_{\gamma} \\
& W_{3 \beta}^{*}=\frac{1}{8 \pi}\left[(2 \ln z-1) n_{\beta}+2 r_{, \beta} r_{, n}\right]-\frac{v}{(1-v) \lambda^{2}} u_{3 \beta \gamma}^{*} n_{\gamma}
\end{aligned}
$$


Conforme (3.11) a influência da carga distribuida " $q(x)$ " na representação integral dos deslocamentos é dada por:

$$
I_{i}=\int_{\Omega_{q}} q(x)\left[u_{i 3}^{*}(\xi, x)-\frac{v}{(1-v) \lambda^{2}} u_{i \alpha, \alpha}^{*}(\xi, x)\right] d \Omega_{q}(x)
$$

A integral " $I_{i}$ " será resolvida agora de forma direta sobre a área carregada " $\Omega_{q}$ ", para um carregamento " $q(x)$ " não mais constante, como foi feito anteriormente, mas com distribuição linear. Para isto considera-se a Fig.3.3:
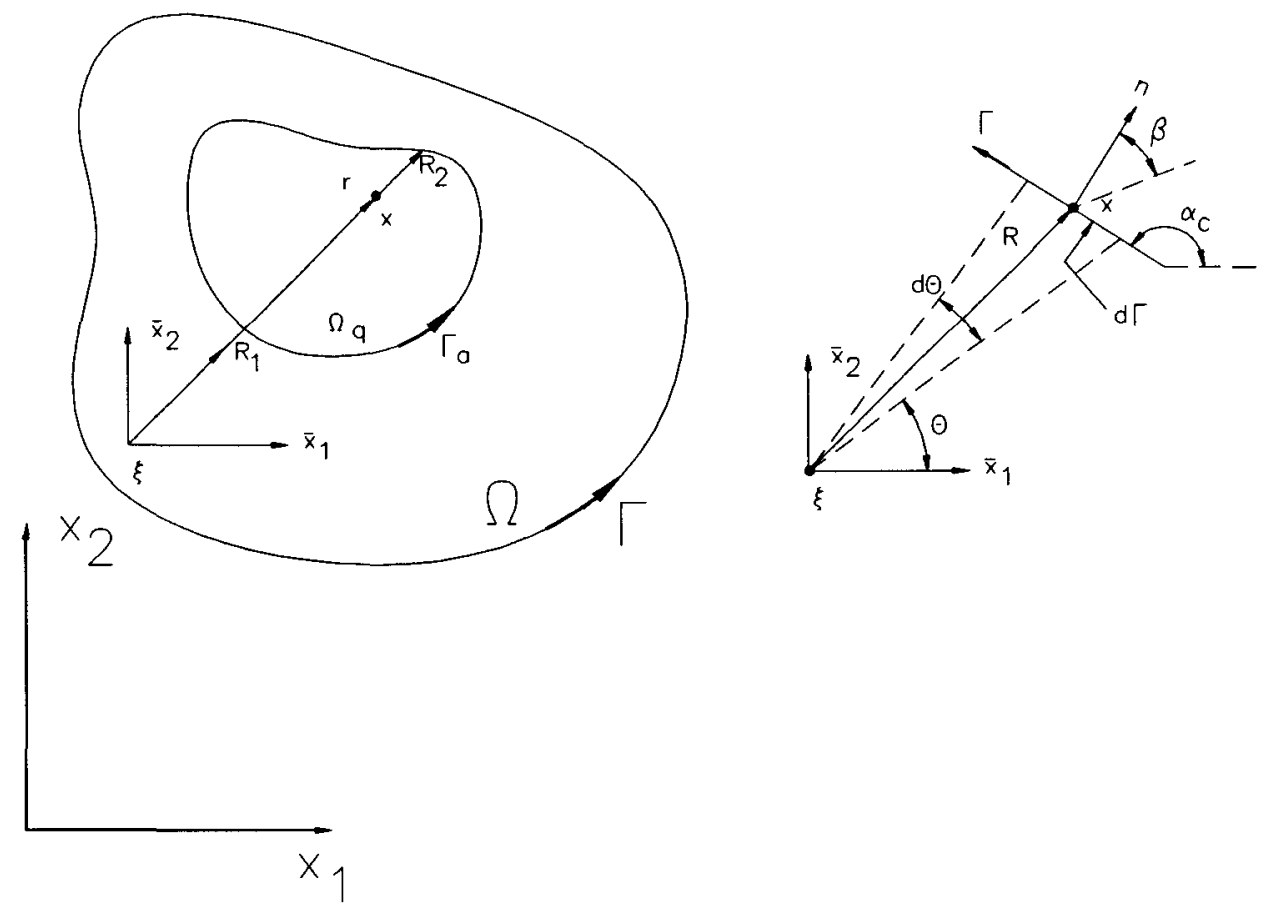

Fig. 3.3 - Área carregada " $\Omega_{q}$ "

O ponto " $\xi$ " é qualquer, podendo ser interno ou externo ao domínio " $\Omega_{q}$ " e o ponto " $x$ " é interno ou no máximo sobre o contorno " $\Gamma_{q}$ ". A distância " $r$ " entre " $\xi$ " e " $x$ " será "R", quando o ponto " $x$ " estiver sobre o contorno " $\Gamma_{q}$ ". 
Observando-se a figura 3.3 pode-se escrever:

$$
\begin{aligned}
& d \Omega_{q}=r d r d \theta \\
& R d \theta=d \Gamma \cos \beta \\
& x_{\alpha}(x)=x_{\alpha}(\xi)+\bar{x}_{\alpha}(\xi) \\
& \bar{x}_{\alpha}(\xi)=r_{r, \alpha}
\end{aligned}
$$

onde $r_{, 1}=\cos \theta$ e $r_{, 2}=\operatorname{sen} \theta$

Assumindo-se variação linear para " $q(x)^{n}$ tem-se:

$$
q(x)=A_{1} x_{1}(x)+B_{1} x_{2}(x)+C_{1}
$$

onde $A_{1}, B_{1}$, e $C_{1}$ são constantes.

Substituindo-se (3.40) em (3.41) obtém-se:

$$
q(x)=\left[A_{1} x_{1}(\xi)+B_{1} x_{2}(\xi)+C_{1}\right]+r\left(A_{1} \cos \theta+B_{1} \operatorname{sen} \theta\right)
$$

Sabendo-se que o termo entre colchetes em (3.42.a) representa 0 valor da carga distribuída no ponto " $\xi$ ", tem-se:

$$
q(x)=q(\xi)+r\left(A_{1} \cos \theta+B_{1} \operatorname{sen} \theta\right)
$$

Levando-se (3.42.b) em (3.38) e sabendo-se que " $q(\xi)$ " é constante, pode-se escrever a integral " $I_{i}$ " da seguinte forma: 


$$
\begin{gathered}
I_{i}=q(\xi) \int_{\Omega_{q}}\left[u_{i 3}^{*}(\xi, x)-\frac{v}{(1-v) \lambda^{2}} u_{i \alpha, \alpha}^{*}(\xi, x)\right] d \Omega_{q}(x)+ \\
\int_{\Omega_{q}}\left(A_{1} \cos \theta+B_{1} \operatorname{sen} \theta\right) r\left[u_{i 3}^{*}(\xi, x)-\frac{v}{(1-v) \lambda^{2}} u_{i \alpha, \alpha}^{*}(\xi, x)\right] d \Omega_{q}(x)
\end{gathered}
$$

As integrais que aparecem em (3.43) envolvem as soluções

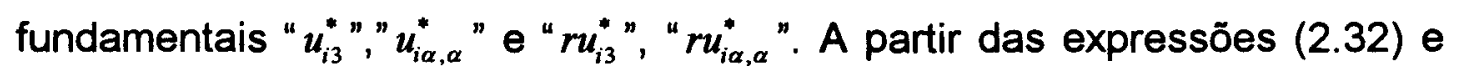
(3.21), transformando-se a integral " $I_{i}$ " (3.43) numa integral dupla em " $r$ " e " $\theta$ " e integrando-se em " $r$ ", quando " $\theta$ " é constante, obtém-se:

$$
\begin{aligned}
& I_{\alpha}=\frac{q(\xi)}{24 \pi D} \int_{\theta}\left[R^{3}\left(2 \ln z-\frac{5}{3}\right)+\frac{12 v}{(1-v) \lambda^{2}} R\right]_{, \alpha} d \theta+ \\
& \frac{1}{32 \pi D} \int_{\theta}\left(A_{1} \cos \theta+B_{1} \operatorname{sen} \theta\right)\left[R^{4}\left(2 \ln z-\frac{3}{2}\right)+\frac{8 v}{(1-v) \lambda^{2}} R^{2}\right]_{, \alpha} d \theta \\
& I_{3}=\frac{q(\xi)}{8 \pi D(1-v) \lambda^{2}} \int_{\theta} R^{2}\left[\frac{(1-v)}{4} z^{2}\left(\ln z-\frac{5}{4}\right)+2(v-2)\left(\ln z-\frac{1}{2}\right)\right] d \theta+ \\
& \frac{1}{8 \pi D(1-v) \lambda^{2}} \int_{\theta}\left(A_{1} \cos \theta+B_{1} \operatorname{sen} \theta\right) R^{3}\left[\frac{(1-v)}{5} z^{2}\left(\ln z-\frac{6}{5}\right)+\frac{4}{3}(v-2)\left(\ln z-\frac{1}{3}\right)\right] d \theta
\end{aligned}
$$

De (3.39) pode-se escrever:

$$
d \theta=\frac{\cos \beta}{R} d \Gamma_{q}
$$

que levado em (3.44.a) fornece a integral " $I_{i}$ ", sobre o contorno " $\Gamma_{\mathrm{q}}$ " da região carregada: 


$$
\begin{aligned}
& I_{\alpha}=\frac{q(\xi)}{24 \pi D} \int_{\Gamma_{q}}\left[R^{2}\left(2 \ln z-\frac{5}{3}\right)+\frac{12 v}{(1-v) \lambda^{2}} r_{, \alpha} \cos \beta d \Gamma_{q}+\right. \\
& \frac{1}{32 \pi D} \int_{\Gamma_{q}}\left(A_{1} \cos \theta+B_{1} \operatorname{sen} \theta\right)\left[R^{3}\left(2 \ln z-\frac{3}{2}\right)+\frac{8 v}{(1-v) \lambda^{2}} R\right]_{, \alpha} \cos \beta d \Gamma_{q}(x) \\
& I_{3}=\frac{q(\xi)}{8 \pi D(1-v) \lambda^{2}} \int_{\Gamma_{q}} R\left[\frac{(1-v)}{4} z^{2}\left(\ln z-\frac{5}{4}\right)+2(v-2)\left(\ln z-\frac{1}{2}\right)\right] \cos \beta d \Gamma_{q}(x)+ \\
& \frac{1}{8 \pi D(1-v) \lambda^{2}} \int_{\Gamma_{q}}\left(A_{1} \cos \theta+B_{1} \operatorname{sen} \theta\right) R^{2}\left[\frac{(1-v)}{5} z^{2}\left(\ln z-\frac{6}{5}\right)+\frac{4}{3}(v-2)\left(\ln z-\frac{1}{3}\right)\right] \\
& \cos \beta d \Gamma_{q}(x)
\end{aligned}
$$

com

$$
\cos \beta=r_{, n}=\cos \theta \operatorname{sen} \alpha_{c}-\operatorname{sen} \theta \cos \alpha_{c}
$$

onde " $\theta$ " e " $\alpha_{c}$ " são respectivamente os ângulos formados pelo raio " $r$ " e o contorno " $\Gamma_{q}$ ", com a direção $x_{1}$.

Será analisada a partir de (3.44.b), a influência desta nova alternativa de distribuição linear de carga " $q(x)$ ", na determinação dos esforços internos " $M_{\alpha \beta}$ " e " $Q_{\alpha}$ ". Efetuando-se as derivadas envolvidas em (2.22) obtém-se para o termo de domínio " $\Omega_{q}$ ":

$$
\begin{array}{ll}
\int_{\Gamma_{q}} q(x) I_{\alpha \beta}^{*}(\xi, x) d \Gamma_{q}(x) & \text { (para momentos) } \\
\int_{\Gamma_{q}} q(x) I_{3 \beta}^{*}(\xi, x) d \Gamma_{q}(x) & \text { (para forças cortantes) }
\end{array}
$$

onde: 


$$
\begin{aligned}
& I_{\alpha \beta}^{*}=-\frac{R}{48 \pi}\left\{4 r_{, \alpha} r_{, \beta} r_{, n}\left(1-v-\frac{12 v}{z^{2}}\right)+\left(2 r_{, n} \delta_{\alpha \beta}+r_{, \alpha} n_{\beta}+r_{, \beta} n_{\alpha}\right)\right. \\
& \left.\left[(1-v)\left(2 \ln z-\frac{5}{3}\right)+\frac{12 v}{z^{2}}\right]+6 r_{, n} \delta_{\alpha \beta}\left[2 \ln z-1+\frac{4 v}{(1-v) z^{2}}\right]\right\}- \\
& \left(A_{1} \cos \theta+B_{1} \operatorname{sen} \theta\right) \frac{R^{2}}{64 \pi}\left\{2 r_{, \alpha} r_{, \beta} r_{, n}\left[(1-v)\left(2 \ln z+\frac{1}{2}\right)-\frac{8 v}{z^{2}}\right]+\right. \\
& \left(2 r_{, n} \delta_{\alpha \beta}+r_{, \alpha} n_{\beta}+r_{, \beta} n_{\alpha}\right)\left[(1-v)\left(2 \ln z-\frac{3}{2}\right)+\frac{8 v}{z^{2}}\right]+ \\
& 8 v r_{, n} \delta_{\alpha \beta}\left[2 \ln z-1+\frac{4 v}{(1-v) z^{2}}\right\} \\
& I_{3 \beta}^{*}=\frac{1}{48 \pi}\left\{r_{, \beta} r_{, n}\left[z^{2} \frac{(1-v)}{2}\left(\ln z-\frac{13}{12}\right)+6(v+2)\right]-\right. \\
& \left.3 n_{, \beta}\left[z^{2} \frac{(1-v)}{4}\left(\ln z-\frac{5}{4}\right)+2(v-2)\left(\ln z-\frac{1}{2}\right)\right]\right\}+\left(A_{1} \cos \theta+B_{1} \operatorname{sen} \theta\right) \frac{R}{64 \pi} \\
& \left\{r_{, \beta} r_{, n}\left[z^{2} \frac{(1-v)}{50}(29-20 \ln z)-\frac{16}{3}(v-2)\left(\ln z-\frac{1}{3}\right)+\frac{8}{3}(v+4)\right]-\right. \\
& \left.4 n_{\beta}\left[\frac{(1-v)}{5} z^{2}\left(\ln z-\frac{6}{5}\right)+\frac{4}{3}(v-2)\left(\ln z-\frac{1}{3}\right)\right]\right\}
\end{aligned}
$$

O termo de domínio " $I_{i}$ " expresso em (3.38) serve também para representar a influência de cargas concentradas e cargas distribuídas em uma linha. No primeiro caso, o termo entre colchetes de (3.38) transformase numa constante e a integral de " $q(x)$ " no domínio " $\Omega_{q}$ ", agora pontual, é a própria carga concentrada " $\mathrm{P}$ ".

Desta forma " $I_{i}$ " se transforma em: 


$$
I_{i}(\xi)=P\left[u_{i 3}^{*}(\xi, x)-\frac{v}{(1-v) \lambda^{2}} u_{i \alpha, \alpha}^{*}(\xi, x)\right]
$$

A expressão (3.48) representa a influência da carga " $P$ " concentrada em " $x$ ", na equação integral dos deslocamentos, para o ponto " $\xi$ ". A influência desta carga " $P$ " no cálculo dos esforços internos " $M_{\alpha \beta}$ " e " $Q_{\alpha}$ " no ponto " $\xi$ ", é dada diretamente, sem integração, pelas equações (3.31).

Para o caso de cargas distribuidas atuando em subdomínios retangulares e alongados, conforme mostrado na Fig. 3.4, é conveniente transformá-las em carregamentos equivalentes, atuando sobre a linha média destes subdomínios. Como " $\mathrm{b}$ " é pequeno quando comparado ao comprimento, pode-se considerar a carga " $q(x)$ " constante na largura. Assim:

$q_{L}(x)=b q(x)$

$$
d \Omega_{q}=b d \Gamma_{q}
$$

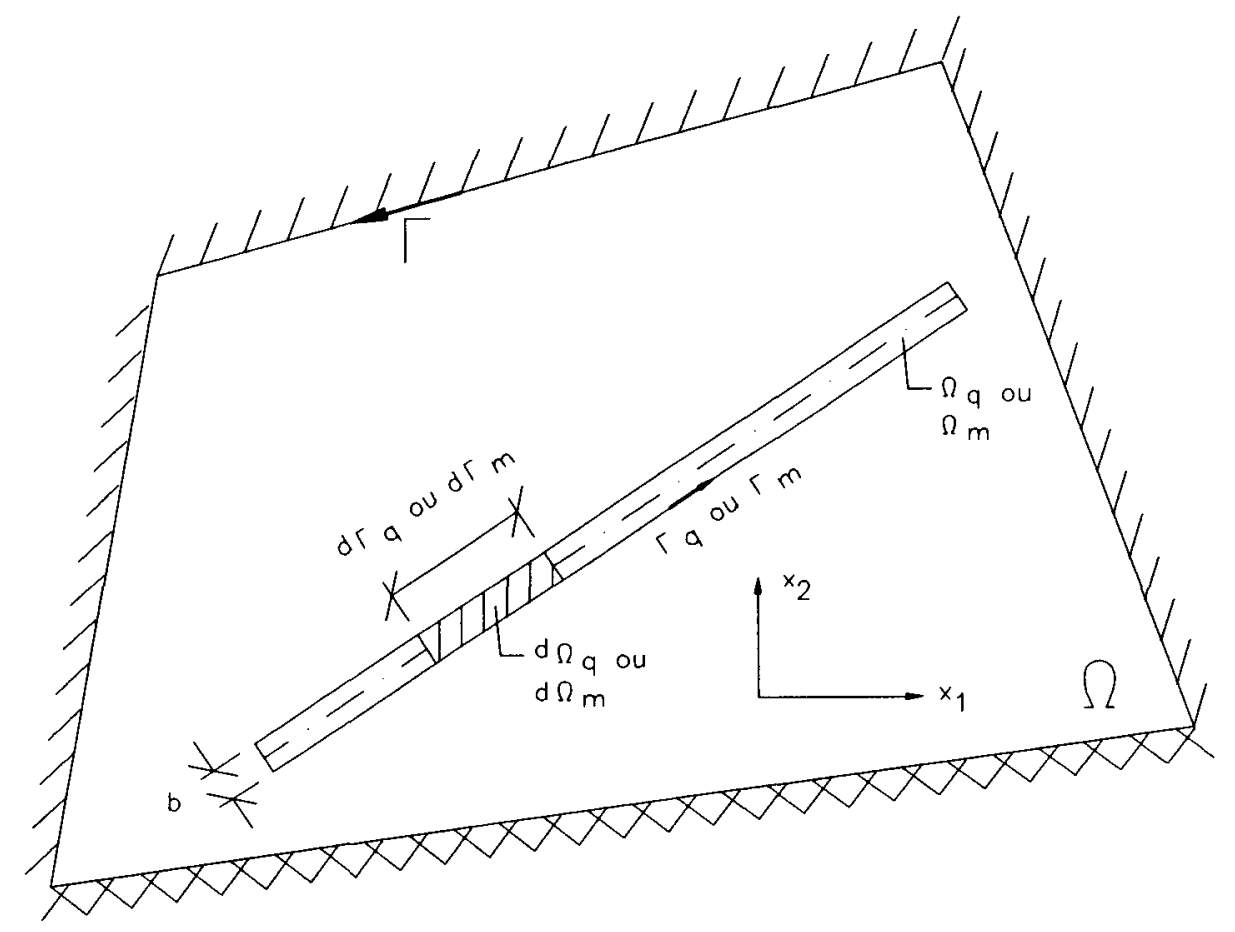

Fig. 3.4 - Cargas distribuidas em subdominios estreitos e alongados 
A situação apresentada na Fig. 3.4 representa a aplicação de uma carga " $\mathrm{q}_{\mathrm{L}}(\mathrm{x})$ " distribuída na linha " $\Gamma_{\mathrm{q}}$ ", ficando a integral " $I_{i}$ " em (3.38) expressa neste caso como:

$$
I_{i}=\int_{\Gamma_{q}} q_{L}(x)\left[u_{i 3}^{*}(\xi, x)-\frac{v}{(1-v) \lambda^{2}} u_{i \alpha, \alpha}^{*}(\xi, x)\right] d \Gamma_{q}(x)
$$

A influência da carga " $q_{L}(x)$ " distribuída em linha na determinação dos esforços internos é dada por:

$$
\int_{\Gamma_{q}} q_{L}(x) w_{i \beta}^{*}(\xi, x) d \Gamma_{q}(x)
$$

com " $w_{i \beta}^{*}(\xi, x)$ " expresso em (3.31).

Caso o ponto fonte " $\xi$ " esteja situado sobre a linha de carga, a integral (3.51) deve ser analisada mais cuidadosamente, devido às singularidades envolvidas no integrando " $w_{i \beta}^{*}$ ", quando o raio " $r$ " tende a zero. Para o levantamento destas singularidades, a linha de carga será acrescida de um contorno circular " $\Gamma_{\varepsilon}$ ", com centro no ponto " $\xi=x$ " e raio " $\varepsilon$ ", conforme Fig. 3.5.

Considerando-se em (3.51) apenas a parcela referente aos momentos internos " $M_{\alpha \beta}$ " $\mathrm{e}$ reescrevendo-a para a situação limite, quando " $\varepsilon$ " tende a zero, obtém-se:

$$
\begin{aligned}
& \lim _{s \rightarrow 0} \int_{\Gamma_{q}-\bar{\Gamma}_{\varepsilon}+\Gamma_{\varepsilon}} q_{L}(x) w_{\alpha \beta}^{*}(\xi, x) d \Gamma_{q}(x)=\lim _{\varepsilon \rightarrow 0} \int_{\Gamma_{q}-\Gamma_{s}} q_{L}(x) w_{\alpha \beta}^{*}(\xi, x) d \Gamma_{q}(x)+ \\
& \lim _{\delta \rightarrow 0} \int_{\Gamma_{\varepsilon}} q_{L}(x) w_{\alpha \beta}^{*}(\xi, x) d \Gamma_{\varepsilon}(x)
\end{aligned}
$$




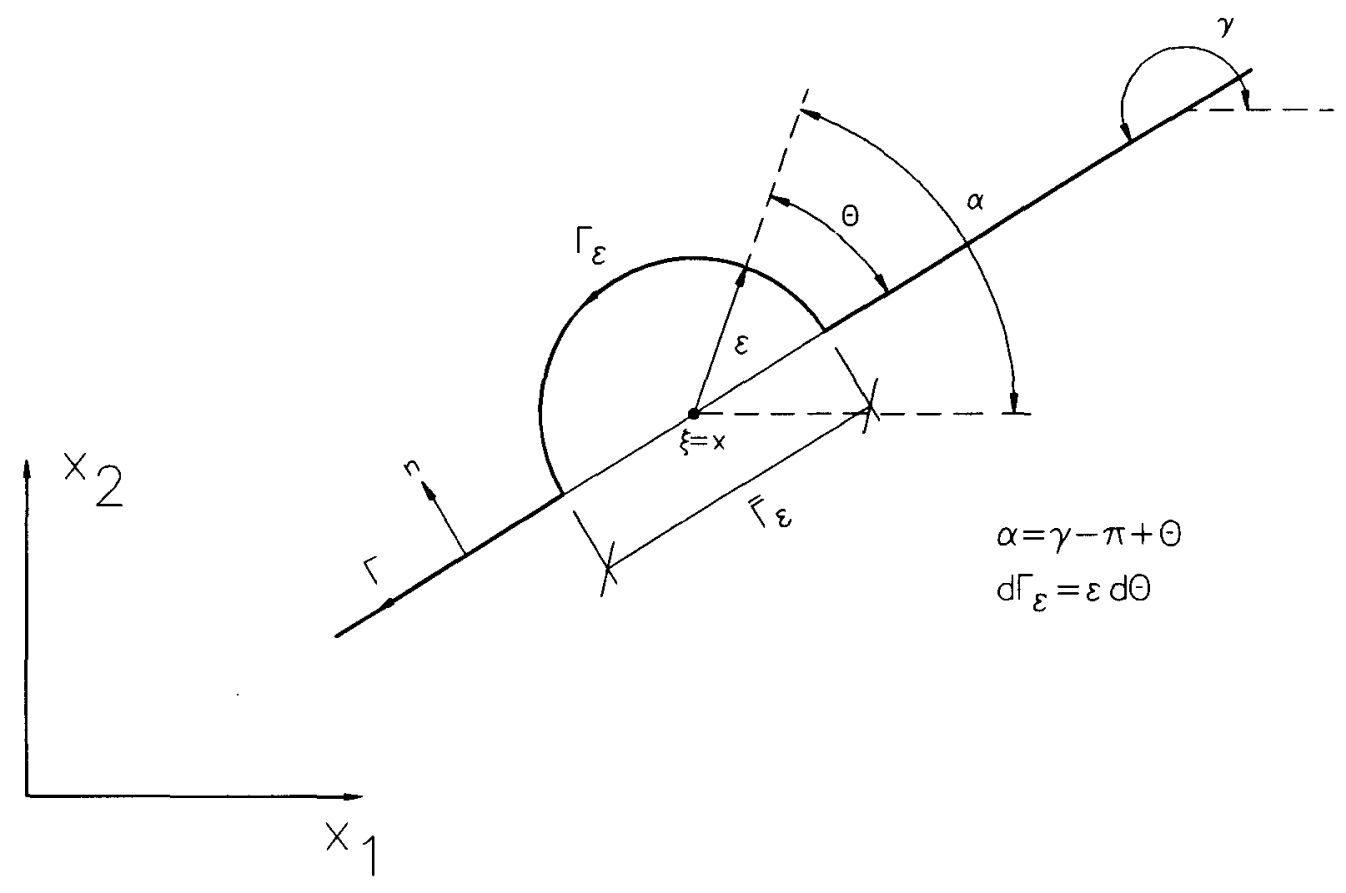

Fig. 3.5 - Linha de carga com contorno alterado.

A primeira parcela do segundo membro de (3.52) deve ser considerada no sentido do valor principal de Cauchy e a sua existência é comprovada desde que a função " $q_{L}(x)$ " satisfaça a condição de Hölder. A segunda parcela será reescrita:

$$
\begin{aligned}
& \lim _{\varepsilon \rightarrow 0} \int_{\Gamma_{\varepsilon}} q_{L}(x) w_{\alpha \beta}^{*}(\xi, x) d \Gamma_{\varepsilon}(x)=\lim _{\varepsilon \rightarrow 0} \int_{\Gamma_{s}} w_{\alpha \beta}^{*}(\xi, x)\left[q_{L}(x)-q_{L}(\xi)\right] d \Gamma_{\varepsilon}(x)+ \\
& q_{L}(\xi) \lim _{\varepsilon \rightarrow 0} \int_{\Gamma_{s}} w_{\alpha \beta}^{*}(\xi, x) d \Gamma_{s}(x)
\end{aligned}
$$

Devido a continuidade da função " $q_{L}(x)$ ", o primeiro termo do segundo membro de (3.53) se anula. Considerando-se a primeira das expressões (3.31) e lembrando-se que quando se integra no contorno " $\Gamma_{\varepsilon}$ " o raio " $r=\varepsilon$ " é constante e o ângulo " $\theta$ " varia de "zero" a " $\pi$ ", obtém-se para a segunda parcela do segundo membro de (3.53): 


$$
\begin{aligned}
& q_{L}(\xi) \lim _{\varepsilon \rightarrow 0} \int_{\Gamma_{s}} w_{\alpha \beta}^{*}(\xi, x) d \Gamma_{\varepsilon}(x)=-\frac{(1-v)}{8 \pi} q_{L}(\xi) \lim _{\varepsilon \rightarrow 0} \int_{0}^{\pi}\left\{\delta_{\alpha \beta}\left[\frac{2(1+v)}{1-v} \ln \lambda \varepsilon-1\right]+\right. \\
& \left.2 r_{, \alpha} r_{, \beta}-\frac{4 v}{(1-v) \lambda^{2} \varepsilon^{2}}\left(2 r_{, \alpha} r_{, \beta}-\delta_{\alpha \beta}\right)\right\} \varepsilon d \theta
\end{aligned}
$$

onde segundo a Fig. 3.5:

$$
\begin{aligned}
& r_{, 1}=\cos \alpha=-\cos \gamma \cos \theta+\operatorname{sen} \gamma \operatorname{sen} \theta \\
& r_{.2}=\operatorname{sen} \alpha=-\operatorname{sen} \gamma \cos \theta-\cos \gamma \operatorname{sen} \theta
\end{aligned}
$$

Substituindo-se (3.54.b) em (3.53) e efetuando-se os limites chega-se a:

$$
q_{L}(\xi) \lim _{s \rightarrow 0} \int_{\Gamma_{s}} w_{\alpha \beta}^{*}(\xi, x) d \Gamma_{\varepsilon}(x)=0
$$

De (3.55) concluiu-se que no cálculo dos momentos internos " $M_{\alpha \beta}$ " a aplicação da carga em linha " $q_{L}(x)$ " não produz descontinuidades, como era de se esperar. Portanto a expressão de (3.52), na situação limite, quando " $\varepsilon$ " tende a zero, " $\left(\Gamma_{q}-\bar{\Gamma}_{\varepsilon}\right)$ " tende a " $\Gamma_{q}$ ", fica:

$$
\lim _{s \rightarrow 0} \int_{\Gamma_{q}-\bar{\Gamma}_{s}+\Gamma_{s}} q_{L}(x) w_{\alpha \beta}^{*}(\xi, x) d \Gamma_{q}(x)=\int_{\Gamma_{q}} q_{L}(x) w_{\alpha \beta}^{*}(\xi, x) d \Gamma_{q}(x)
$$

A integral (3.56) será estudada posteriormente no item 3.3.5, quando a linha de carga estará dividida em elementos.

Analisando-se (3.51) para a segunda das expressões (3.31), “ $w_{3 \beta}^{*}$ ", obtém-se a contribuição da carga distribuída " $\mathrm{q}_{\mathrm{L}}(\mathrm{x})$ " na determinação das 
forças cortantes " $Q_{\alpha}$ ". Quando o ponto " $\xi$ " coincide com a linha de carga, deve-se fazer um estudo análogo ao que foi feito para o caso dos momentos, alterando-se o contorno " $\Gamma_{\mathrm{q}}$ " conforme a Fig. 3.5. A integral (3.51) no limite quando " $\varepsilon$ " tende a zero será dada por:

$$
\begin{aligned}
& \lim _{\varepsilon \rightarrow 0} \int_{\Gamma_{q}-\bar{\Gamma}_{\tau_{s}}+\Gamma_{\varepsilon}} q_{L}(x) w_{3 \beta}^{*}(\xi, x) d \Gamma_{q}(x)=\lim _{\varepsilon \rightarrow 0} \int_{\Gamma_{q}-\bar{\Gamma}_{q}} q_{L}(x) w_{3 \beta}^{*}(\xi, x) d \Gamma_{q}(x)+ \\
& \lim _{\varepsilon \rightarrow 0} \int_{\Gamma_{s}} q_{L}(x) w_{3 \beta}^{*}(\xi, x) d \Gamma_{\varepsilon}(x)
\end{aligned}
$$

A primeira parcela do segundo membro de (3.57) deve ser calculada no sentido do valor principal de Cauchy e a segunda pode ser reescrita como:

$$
\begin{aligned}
& \lim _{\delta \rightarrow 0} \int_{\Gamma_{\varepsilon}} q_{L}(x) w_{3 \beta}^{*}(\xi, x) d \Gamma_{\varepsilon}(x)=\lim _{\varepsilon \rightarrow 0} \int_{\Gamma_{s}} w_{3 \beta}^{*}(\xi, x)\left[q_{L}(x)-q_{L}(\xi)\right] d \Gamma_{\delta}(x)+ \\
& q_{L}(\xi) \lim _{s \rightarrow 0} \int_{\Gamma_{s}} w_{3 \beta}^{*}(\xi, x) d \Gamma_{\varepsilon}(x)
\end{aligned}
$$

Para " $q_{L}(x)$ " contínua, a primeira parcela do segundo membro de (3.58) se anula. O limite da segunda parcela será calculado a partir de (3.31) lembrando-se que na integração sobre " $\Gamma_{\varepsilon}$ ", o raio é constante e igual a " $\varepsilon$ ", enquanto " $\theta$ " varia de zero a " $\pi$ ". Assim obtém-se:

$$
q_{L}(\xi) \lim _{\varepsilon \rightarrow 0} \int_{\Gamma_{s}} w_{3 \beta}^{*}(\xi, x) d \Gamma_{\varepsilon}(x)=q_{L}(\xi) \lim _{\varepsilon \rightarrow 0} \int_{0}^{\pi} \frac{r_{, \beta}}{2 \pi \varepsilon} \varepsilon d \theta
$$

com " $r, \beta$ " dado em (3.54.b).

Efetuando-se o limite em (3.59) obtém-se: 


$$
q_{L}(\xi) \lim _{\varepsilon \rightarrow 0} \int_{\Gamma_{s}} w_{3 \beta}^{*}(\xi, x) d \Gamma_{\varepsilon}(x)=q_{L}(\xi) \frac{n_{\beta}}{\pi}
$$

onde:

$$
\begin{aligned}
& n_{1}=\cos \left(\gamma-\frac{\pi}{2}\right)=\operatorname{sen} \gamma \\
& n_{2}=\operatorname{sen}\left(\gamma-\frac{\pi}{2}\right)=-\cos \gamma
\end{aligned}
$$

são os cossenos diretores do vetor " $n$ ", normal ao contorno " $\Gamma_{q}$ ".

Finalmente a contribuição da linha de carga " $q_{L}(x)^{n}$, no cálculo das forças cortantes, quando o ponto fonte coincide com a mesma, será dada por:

$$
q_{L}(\xi) \frac{n_{\beta}}{\pi}+\int_{\Gamma_{q}} q_{L}(x) w_{3 \beta}^{*}(\xi, x) d \Gamma_{q}(x)
$$

A integral em (3.62) será analisada posteriormente no item 3.3.5, quando " $\Gamma_{\mathrm{q}}$ " será dividido em elementos.

Conforme será visto no capítulo 4, é de interesse para este trabalho o estudo da influência da aplicação de linhas de momentos, na determinação de deslocamentos e esforços internos. Por isto será analisado apenas a aplicação de momentos externos distribuidos em subdomínios estreitos e alongados, conforme ilustrado na Fig. 3.4. Desta forma, as equações (3.49) transformam-se em:

$$
\begin{aligned}
& m_{\alpha}^{L}(x)=b m_{\alpha}(x) \\
& d \Omega_{m}(x)=b d \Gamma_{m}(x)
\end{aligned}
$$


A influência da aplicação dos momentos distribuídos em linha " $m_{\alpha}^{L}(x)$ " no cálculo dos deslocamentos, equações (3.11) e (3.15), pode ser expressa por:

$$
\int_{\Gamma_{m}} m_{\alpha}^{L}(x) u_{i \alpha}^{*}(\xi, x) d \Gamma_{m}(x)
$$

No cálculo dos esforços internos " $M_{\alpha \beta}$ " e " $Q_{\alpha}$ ", a aplicação de linhas de momentos, leva a uma transformação da integral sobre o subdomínio " $\Omega_{m}$ " em (3.26) e (3.27), para uma integral sobre o contorno " $\Gamma_{m}$ ", conforme Fig.3.4. Assim, escreve-se:

$$
\int_{\Omega_{m}} m_{r}(x) u_{i \beta r}^{*}(\xi, x) d \Omega_{m}(x)=\int_{\Gamma_{m}} m_{r}^{L}(x) u_{i \beta r}^{*}(\xi, x) d \Gamma_{m}(x)
$$

Caso o ponto fonte coincida com a linha de momentos, considera-se de forma análoga, o contorno alterado, conforme Fig. 3.5. Analisando-se primeiramente o cálculo dos momentos, a integral do segundo membro de (3.65), deve ser escrita na situação limite, quando " $\varepsilon$ " tende a zero, por:

$$
\begin{aligned}
& \lim _{\varepsilon \rightarrow 0} \int_{\Gamma_{\Gamma_{m}}-\bar{\Gamma}_{s}+\Gamma_{s}} m_{\gamma}^{L}(x) u_{\alpha \beta \gamma}^{*}(\xi, x) d \Gamma_{m}(x)=\lim _{\varepsilon \rightarrow 0} \int_{\Gamma_{\Gamma_{m}-\Gamma_{s}}} m_{\gamma}^{L}(x) u_{\alpha \beta \gamma}^{*}(\xi, x) d \Gamma_{m}(x)+ \\
& \lim _{\varepsilon \rightarrow 0} \int_{\Gamma_{s}} m_{y}^{L}(x) u_{\alpha \beta \gamma}^{*}(\xi, x) d \Gamma_{m}(x)
\end{aligned}
$$

Caso " $m_{\alpha}^{L}(x)$ " satisfaça a condição de Hölder, a primeira parcela do segundo membro de (3.66) deverá ser calculada no sentido do valor principal de Cauchy. A segunda parcela pode ser reescrita como:

$$
\begin{aligned}
& \lim _{\varepsilon \rightarrow 0} \int_{\Gamma_{s}} m_{\gamma}^{L}(x) u_{\alpha \beta \gamma}^{*}(\xi, x) d \Gamma_{m}(x)=\lim _{\varepsilon \rightarrow 0} \int_{\Gamma_{s}}\left[m_{r}^{L}(x)-m_{r}^{L}(\xi)\right] u_{\alpha \beta \gamma}^{*}(\xi, x) a \Gamma_{m}(x)+ \\
& m_{r}^{L}(\xi) \lim _{s \rightarrow 0} \int_{\Gamma_{s}} u_{\alpha \beta \gamma}^{*}(\xi, x) d \Gamma_{m}(x)
\end{aligned}
$$


Devido à continuidade de " $m_{r}^{L}(x)$ " a primeira parcela do segundo membro de (3.67) se anula. O limite envolvido na segunda parcela será determinado a partir da primeira das equaçōes (3.29), lembrando-se que quando o raio " $r$ " tende a zero, os valores de " $A$ " e " $z k_{1}$ " tendem respectivamente, para $-0,5$ e 1 (Ver apêndice A). Além disso, quando se integra sobre " $\Gamma_{\varepsilon}$ " $r$ " é constante e " $\theta$ " varia de "zero" a " $\pi$ ". Assim pode-se escrever:

$$
\begin{aligned}
& m_{\gamma}^{L}(\xi) \lim _{\varepsilon \rightarrow 0} \int_{\Gamma_{s}} u_{\alpha \beta \gamma}^{*}(\xi, x) d \Gamma_{m}(x)= \\
& m_{\gamma}^{L}(\xi) \lim _{\varepsilon \rightarrow 0} \frac{1}{4 \pi \varepsilon} \int_{0}^{\pi}\left[(1-v)\left(r_{, \alpha} \delta_{\beta \gamma}+r_{, \beta} \delta_{\alpha \gamma}-r_{, \gamma} \delta_{\alpha \beta}\right)+2(1+v) r_{, \alpha} r_{, \beta} r_{, \gamma}\right] \varepsilon d \theta
\end{aligned}
$$

Para os valores de " $r, \alpha$ " definidos em (3.54) pode-se escrever os limites em (3.68), de forma explícita:

$$
\begin{aligned}
& \lim _{s \rightarrow 0} \int_{\Gamma_{s}} u_{111}^{*}(\xi, x) d \Gamma_{m}(x)=\frac{1}{2 \pi}\left[(1-v) \operatorname{sen} \gamma+2(1+v)\left(\operatorname{sen} \gamma-\frac{\operatorname{sen}^{3} \gamma}{3}\right)\right] \\
& \lim _{\varepsilon \rightarrow 0} \int_{\Gamma_{s}} u_{112}^{*}(\xi, x) d \Gamma_{m}(x)=\frac{1}{2 \pi}\left[(1-v) \cos \gamma-\frac{2}{3}(1+v) \cos ^{3} \gamma\right] \\
& \lim _{s \rightarrow 0} \int_{\Gamma_{s}} u_{121}^{*}(\xi, x) d \Gamma_{m}(x)=\frac{1}{2 \pi}\left[-(1-v) \cos \gamma-\frac{2}{3}(1+v) \cos ^{3} \gamma\right] \\
& \lim _{\varepsilon \rightarrow 0} \int_{\Gamma_{s}} u_{122}^{*}(\xi, x) d \Gamma_{m}(x)=\frac{1}{2 \pi}\left[(1-v) \operatorname{sen} \gamma+\frac{2}{3}(1+v) \operatorname{sen}^{3} \gamma\right] \\
& \lim _{\varepsilon \rightarrow 0} \int_{\Gamma_{s}} u_{221}^{*}(\xi, x) d \Gamma_{m}(x)=\frac{1}{2 \pi}\left[-(1-v) \operatorname{sen} \gamma+\frac{2}{3}(1+v) \operatorname{sen}^{3} \gamma\right]
\end{aligned}
$$




$$
\lim _{\varepsilon \rightarrow 0} \int_{\Gamma_{c}} u_{222}^{*}(\xi, x) d \Gamma_{m}(x)=\frac{1}{2 \pi}\left[-(1-v) \cos \gamma-2(1+v)\left(\cos \gamma-\frac{\cos ^{3} \gamma}{3}\right)\right]
$$

A contribuição da linha de momentos, na determinação das forças cortantes é feita substituindo-se na equação (3.65) a letra " $i$ " pelo número "3" nos valores " $u_{i \beta y}^{*}$ ". Assim:

$$
\int_{\Omega_{*}} m_{\gamma}(x) u_{3 \beta \gamma}^{*}(\xi, x) d \Omega_{m}(x)=\int_{\Gamma_{m}} m_{\gamma}^{L}(x) u_{3 \beta \gamma}^{*}(\xi, x) d \Gamma_{m}(x)
$$

Caso o ponto fonte coincida com a linha de momentos, procede-se de forma análoga ao efetuado no cálculo dos momentos, alterando-se o contorno, conforme a Fig. 3.5, ficando o segundo membro de (3.70) expresso na situação limite quando " $\varepsilon$ " tende a "zero", por:

$$
\begin{aligned}
& \lim _{\varepsilon \rightarrow 0} \int_{\Gamma_{\Gamma_{m}}-\bar{\Gamma}_{s}+\Gamma_{s}} m_{r}^{L}(x) u_{3 \beta \gamma}^{*}(\xi, x) d \Gamma_{m}(x)=\lim _{\delta \rightarrow 0} \int_{\Gamma_{\Gamma_{m}}-\bar{\Gamma}_{s}} m_{r}^{L}(x) u_{3 \beta r}^{*}(\xi, x) d \Gamma_{m}(x)+ \\
& \lim _{\delta \rightarrow 0} \int_{\Gamma_{s}} m_{\gamma}^{L}(x) u_{3 \beta r}^{*}(\xi, x) d \Gamma_{\delta}(x)
\end{aligned}
$$

A primeira parcela do segundo membro de (3.71) será calculada no sentido do valor principal de Cauchy, sendo sua existência comprovada caso " $m_{y}^{L}(x)$ " satisfaça a condição de Hölder. A segunda parcela pode ser escrita como:

$$
\begin{aligned}
& \lim _{\varepsilon \rightarrow 0} \int_{\Gamma_{\varepsilon}} m_{r}^{L}(x) u_{3 \beta \gamma}^{*}(\xi, x) d \Gamma_{\varepsilon}(x)=\lim _{\varepsilon \rightarrow 0} \int_{\Gamma_{\varepsilon}}\left[m_{r}^{L}(x)-m_{r}^{L}(\xi)\right] u_{3 \beta \gamma}^{*}(\xi, x) d \Gamma_{\varepsilon}(x)+ \\
& m_{r}^{L}(\xi) \lim _{\varepsilon \rightarrow 0} \int_{\Gamma_{\varepsilon}} u_{3 \beta_{r}}^{*}(\xi, x) d \Gamma_{\varepsilon}(x)
\end{aligned}
$$

Aqui mais uma vez admitindo-se a continuidade da função " $m_{y}^{L}(x)$ ", a primeira parcela do segundo membro de (3.72) se anula. Analisando-se 
" $u_{3 \beta r}^{*}$ " em (3.29) e sabendo-se que quando " $r$ " tende a "zero" os valores de " $B$ " e " $A$ " tendem respectivamente a [-0,5 In (z/2) - 0,5386078] e -0,5, podese escrever para a segunda parcela:

$$
\lim _{s \rightarrow 0} \int_{0}^{\pi} \frac{\lambda^{2}}{2 \pi}\left[-\delta_{\beta r}\left(\frac{1}{2} \ln \frac{\lambda \varepsilon}{2}-0,5386078\right)-\frac{1}{2} r_{\beta} r_{, r}\right] \varepsilon d \theta
$$

O valor do limite (3.73) é igual a "zero" ficando a contribuição dos momentos em linha, no cálculo das forças cortantes, dado apenas como o primeiro termo de (3.71). Tanto este termo como o primeiro de (3.66) serão analisados no item 3.3.5, quando a linha de momentos será dividida em elementos.

\section{3 - MÉTODO DOS ELEMENTOS DE CONTORNO}

\subsection{1 - CONSIDERAÇÕES INICIAIS}

No item 3.2 foram desenvolvidas equaçöes integrais para os deslocamentos e esforços internos, relacionando-se deslocamentos e forças de superfície no contorno.

A solução analítica para estas equações limita a aplicação a poucos problemas clássicos, com solução conhecida. A maioria dos problemas práticos de aplicação de placas não tem solução analítica conhecida, cabendo à solução numérica aproximada, a alternativa viável para a resolução dos mesmos. Dividindo-se o contorno da placa em "segmentos", denominados elementos de contorno, onde os deslocamentos e forças de superfície serão aproximados por funções previamente estabelecidas, 
transforma-se o sistema de equações integrais em um sistema de equações algébricas lineares. De forma análoga transformam-se as integrais de domínio em integrais sobre o contorno dos subdomínios carregados.

As equações algébricas resultantes da transformação das equaçōes integrais para todos os pontos do contorno, dão origem a um sistema de equações lineares, onde as incógnitas são os próprios deslocamentos e forças de superfície no contorno. A utilização da Teoria de Reissner aplicada à placas, conduz a três equações para cada ponto de colocação, duas correspondentes às rotações normal e tangencial ao contorno e uma relativa ao deslocamento transversal.

Impondo-se as condiçōes de contorno, o sistema é resolvido e em seguida, são calculados de forma direta, os deslocamentos e esforços em pontos internos.

\subsection{2 - DISCRETIZAÇÃO DAS EQUAÇŌES INTEGRAIS DE CONTORNO.}

A equaçăo (3.15) representa os deslocamentos generalizados para pontos do contorno, em funçăo dos deslocamentos e forças de superfície, de todos os pontos do contorno " $\Gamma^{n}$. Ao dividir o contorno em elementos e aproximar os deslocamentos e forças no contorno, por funções de interpolação previamente escolhidas, estas variáveis podem ser escritas em função de seus valores discretos, em pontos denominados nós dos elementos. Desta forma consegue-se discretizar a equação integral (3.15).

Para uma placa com contorno qualquer, o número e a forma dos elementos são escolhidos, usando-se a discretização mais adequada para representar o contorno real. 
Neste trabalho o contorno real é aproximado por uma série de elementos de contorno retos, necessitando-se apenas dois pontos (nós) para definir geometricamente cada elemento (Fig. 3.6). Com o intuito de facilitar a integração numérica, as coordenadas de todos os pontos do elemento serão parametrizadas em função das coordenadas dos nós extremos, através de coordenadas locais homogêneas " $\eta$ " (Fig. 3.7).

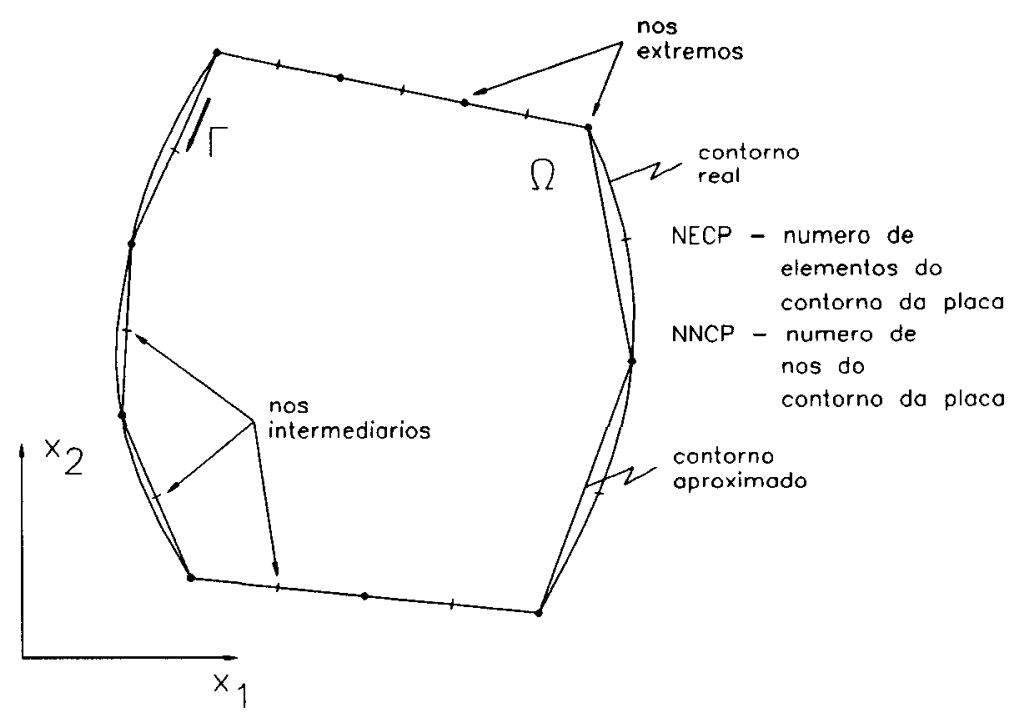

Fig. 3.6 - Discretização do contorno.

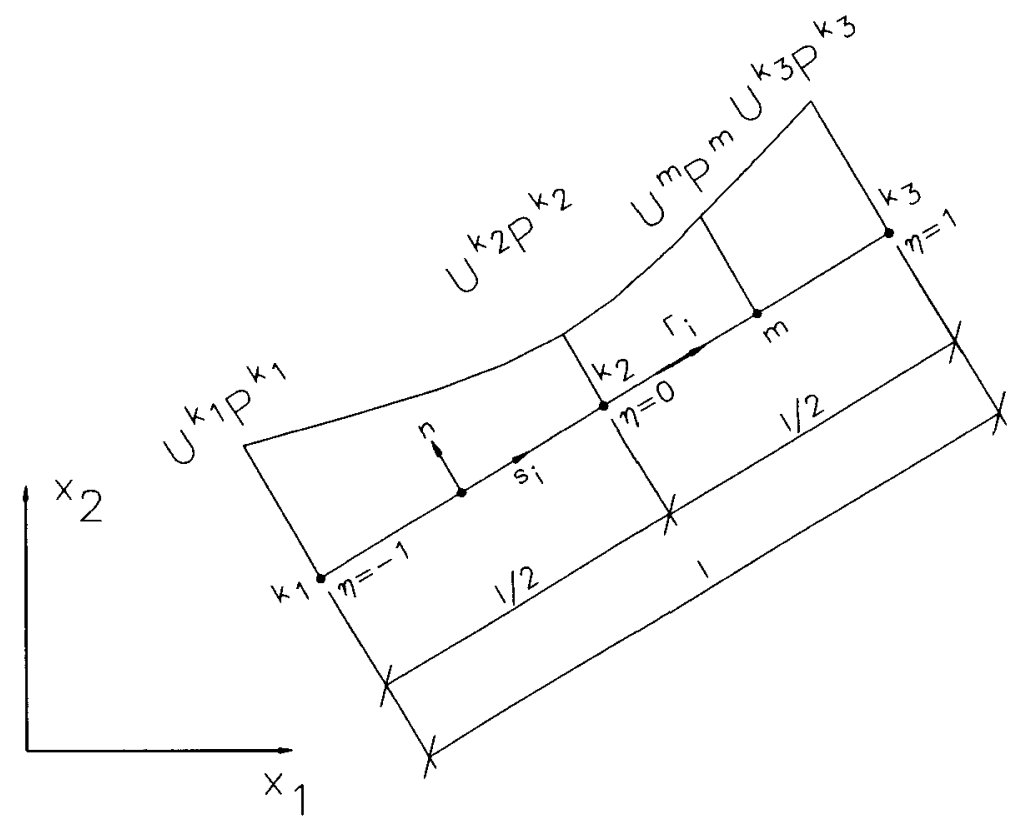

Fig. 3.7 - Elemento de contorno " $\Gamma_{i}$ " 
Para um ponto genérico " $m$ " sobre o contorno " $\Gamma_{i}$ p pode-se escrever:

$$
{\underset{\sim}{x}}^{m}=\stackrel{*}{*}(\eta) \underset{\sim}{X^{N}}
$$

onde:

$$
\begin{aligned}
& \stackrel{\sim}{m}^{m}=\left\{\begin{array}{l}
x_{1} \\
x_{2}
\end{array}\right\}^{m}
\end{aligned}
$$

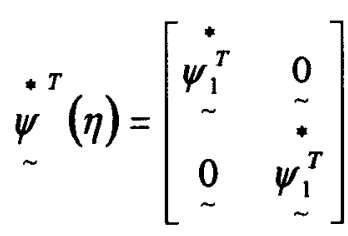

$$
\begin{aligned}
& X_{\sim}^{N}=\left\{\begin{array}{c}
X_{1}^{k_{1}} \\
X_{1}^{k_{3}} \\
X_{2}^{k_{1}} \\
X_{2}^{k_{3}}
\end{array}\right\} \\
& \dot{\psi}_{\sim}=\left\{\begin{array}{l}
\phi_{1}^{*} \\
\phi_{2}^{*}
\end{array}\right\}=\frac{1}{2}\left\{\begin{array}{l}
1-\eta \\
1+\eta
\end{array}\right\}
\end{aligned}
$$

Os deslocamentos e forças no contorno serão interpolados por funçōes quadráticas, sendo necessário a definição de mais um nó, " $k_{2}$ ", escolhido no centro de cada elemento. Assim os valores de contorno poderão ser escritos em função dos valores nos nós " $k_{1}$ ", "k" e " $k_{3}$ ", da seguinte forma:

$$
\begin{aligned}
& {\underset{\sim}{u}}^{m}=\underset{\sim}{\psi^{T}}(\eta) \underset{\sim}{U^{N}} \\
& \sim_{\sim}^{m}={\underset{\sim}{\Psi}}^{T}(\eta) \underset{\sim}{P^{N}}
\end{aligned}
$$

onde: 


$$
\stackrel{\sim}{u}^{m}=\left\{\begin{array}{l}
u_{1}=\phi_{n} \\
u_{2}=\phi_{s} \\
u_{3}=w
\end{array}\right\}
$$

$$
\tilde{p}^{m}=\left\{\begin{array}{c}
p_{1}=M_{n} \\
p_{2}=M_{n s} \\
p_{3}=Q_{n}
\end{array}\right\}^{m}
$$

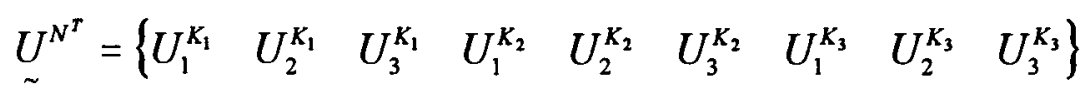

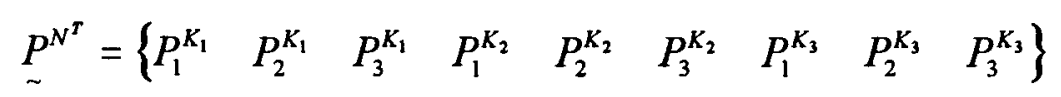

$$
\underset{\sim}{\psi^{T}}(\eta)=\left[\begin{array}{ccc}
\psi_{1} & \psi_{2} & \psi_{3} \\
\sim & \sim & \sim
\end{array}\right]
$$$$
\psi_{1}(\eta)=\left[\begin{array}{ccc}
\phi_{1} & 0 & 0 \\
0 & \phi_{1} & 0 \\
0 & 0 & \phi_{1}
\end{array}\right], \psi_{2}(\eta)=\left[\begin{array}{ccc}
\phi_{2} & 0 & 0 \\
0 & \phi_{2} & 0 \\
0 & 0 & \phi_{2}
\end{array}\right], \psi_{3}(\eta)=\left[\begin{array}{ccc}
\phi_{3} & 0 & 0 \\
0 & \phi_{3} & 0 \\
0 & 0 & \phi_{3}
\end{array}\right]
$$

$$
\begin{gathered}
\phi_{1}(\eta)=\frac{1}{2} \eta(\eta-1) \\
\phi_{2}(\eta)=(1+\eta)(1-\eta) \\
\phi_{3}(\eta)=\frac{1}{2} \eta(\eta+1)
\end{gathered}
$$

Em (3.77) " $\phi_{n}{ }^{n}$ e " $\phi_{s}$ " são respectivamente as rotações nos planos normais " $x_{3} n$ " e " $x_{3} s$ "; " $w$ " é o deslocamento transversal na direção " $x_{3}$ ", para 
pontos do contorno. " $M_{n}$ ", " $M_{n s}$ " $e$ " $Q_{n}$ " são os respectivos esforços no contorno associadas aos deslocamentos " $\phi_{n}$ ", " $\phi_{s}{ }^{n} \mathrm{e}$ "W".

Considerando-se a discretização do contorno, conforme a Fig. 3.6, a equação (3.15) pode ser escrita como:

$$
\begin{aligned}
& c_{i j}(\chi) u_{j}(\chi)=\sum_{k=1}^{N E C P} \int_{\Gamma_{k}} u_{i j}^{*}(\chi, X) p_{j}(X) d \Gamma_{k}(X)- \\
& \sum_{k=1}^{N E C P} \int_{\Gamma_{k}} p_{i j}^{*}(\chi, X) u_{j}(X) d \Gamma_{k}(X)+ \\
& \int_{\Omega_{q}} q(x)\left[u_{i 3}^{*}(\chi, x)-\frac{v}{(1-v) \lambda^{2}} u_{i \alpha, \alpha}^{*}(\chi, x)\right] d \Omega_{q}(x)+ \\
& \int_{\Omega_{m}} m_{\alpha}(x) u_{i \alpha}^{*}(\chi, x) d \Omega_{m}(x)
\end{aligned}
$$

onde "NECP" representa o número de elementos do contorno da placa.

Mudando-se a variável " $\Gamma$ " para " $\eta$ " conforme:

$$
\begin{aligned}
& \Gamma=\frac{l}{2} \eta \\
& |J|=\frac{d \Gamma}{d \eta}=\frac{l}{2}
\end{aligned}
$$

e escrevendo-se " $u_{j}$ " e " $p_{j}$ " dados em (3.76), pode-se escrever (3.80) como:

$$
\begin{aligned}
& c_{i j}(\chi) u_{j}(\chi)=\sum_{k=1}^{N E C P} \int_{-1}^{+1}|J| u_{i j}^{*}(\chi, X) \underset{\sim}{\psi_{\sim}^{T}(\eta) d \eta(X) P_{j(k)}^{N}-} \\
& \sum_{k=1}^{N E C P} \int_{-1}^{+1}|J| p_{i j}^{*}(\chi, X) \psi_{\sim}^{T}(\eta) d \eta(X) U_{j(k)}^{N}+
\end{aligned}
$$




$$
\begin{aligned}
& \int_{\Omega_{q}} q(x)\left[u_{i 3}^{*}(\chi, x)-\frac{v}{(1-v) \lambda^{2}} u_{i \alpha, \alpha}^{*}(\chi, x)\right] d \Omega_{q}(x)+ \\
& \int_{\Omega_{m}} m_{\alpha}(x) u_{i \alpha}^{*}(x, x) d \Omega_{m}(x)
\end{aligned}
$$

A equação (3.82), quando escrita para todos os "NNCP" pontos nodais do contorno, representa um sistema de "3(NNCP)" equações algébricas lineares, onde as incógnitas são os valores nodais " $U_{j(k)}^{N}$ " ou " $P_{j(k)}^{N}$ ". As integrais sobre os elementos exprimem a influência dos valores nodais de deslocamentos e forças no contorno, sobre os deslocamentos nos pontos fonte " $\chi$ ". Estas integrais são do tipo:

$$
\begin{aligned}
& \int_{-1}^{+1}|J| u_{i j}^{*}(\chi, X) \psi_{\sim}^{T}(\eta) d \eta(X) \\
& \int_{-1}^{+1}|J| p_{i j}^{*}(\chi, X) \psi_{\sim}^{T}(\eta) d \eta(X)
\end{aligned}
$$

As integrais (3.83) dependem apenas das soluçōes fundamentais " $u_{i j}^{*}$ e " $p_{i j}^{*}$, e das funções aproximadoras " $\psi^{\mathrm{T}}$. Como as soluções fundamentais são função de " $r$ ", que depende da posição do ponto " $X$ ", agora função de " $\eta$ ", os integrandos de (3.83) são também função de " $\eta$ ", podendo-se efetuar a integração numérica, que além de simples, é eficiente para o tratamento computacional do método. A partir de (3.83) pode-se escrever para cada um dos três pontos nodais do elemento " $\Gamma_{k}$ ":

$$
\begin{aligned}
& g_{i j}^{n}(\chi)=\frac{l_{k}}{2} \int_{-1}^{+1} u_{i j}^{*}(\chi, X) \psi_{n}(\eta) d \eta(X) \\
& h_{i j}^{n}(\chi)=\frac{l_{k}}{2} \int_{-1}^{+1} p_{i j}^{*}(\chi, X) \psi_{n}(\eta) d \eta(X)
\end{aligned}
$$


onde:

" $u_{i j}^{*}$ e " $p_{i j}^{*} "$ - são as soluções fundamentais generalizadas em deslocamentos e forças de superfície, dadas respectivamente nas equações (2.32) e (3.22).

"n" - representa o nó local do elemento onde se mede a resposta ao carregamento unitário aplicado.

" $\chi$ " - ponto de colocação, isto é, o ponto onde é aplicada a carga unitária ou para o qual se escrevem as equações.

"i" - indica qual a equação está sendo gerada para o ponto " $\chi$ ". Se "i"=1,2 são geradas respectivamente as equações para as rotações " $\phi_{n}$ " $e$ " $\phi_{s}$ " e se " $i "=3$, a equação do deslocamento " $w$ ". Em outras palavras, "i" indica a ação unitária aplicada em " $\chi$ ".

"j" - indica a natureza da resposta medida em " $X$ ". Se "j"=1 a resposta fundamental é relativa ao momento " $M_{n}^{*}$ " ou ao correspondente deslocamento " $\phi_{n}^{*}$; se "i" $=2$ a resposta é relativa ao momento " $M_{n s}^{*}$ " ou ao correspondente " $\phi_{s}^{*}$; se "i" $=3$ a resposta é relativa a força " $Q_{n}^{*}$ ou ao respectivo deslocamento " $w$ " .

Nota-se que para nós " $n$ " extremos, os coeficientes (3.84) recebem a contribuição dos dois elementos adjacentes.

Gerando-se (3.84) para os três pontos nodais de todos os "NECP" elementos, endereçando-se e acumulando-se convenientemente, obtém-se as matrizes " $G$ " e "H", que são as matrizes de influência dos valores nodais, do Método dos Elementos de Contorno. Assim, o sistema obtido a partir de (3.82), com "3*NNCP" equaçōes, pode ser representado matricialmente como se segue: 


$$
\underset{\sim}{C} \underset{\sim}{U}+\underset{\sim}{H} \underset{\sim}{U}=\underset{\sim}{G} \underset{\sim}{P}+\underset{\sim}{B}
$$

onde:

" $\hat{H}^{\prime} e^{\prime} \underline{\sim}$ - são as matrizes de influência

" $U^{U} e^{\prime} \stackrel{\sim}{P}^{n}-\quad$ são respectivamente os vetores dos deslocamentos e forças de superfície, para todos os pontos nodais do contorno.

" B $^{n}$ - vetor contendo a influência de todo o carregamento de domínio, cargas e momentos distribuídos.

A matriz " $C$ ", conforme mostrado em 3.2.2, depende apenas da geometria do contorno em cada ponto " $\chi$ ", podendo-se incorporá-la à matriz " $\hat{H}$ ", resultando:

$$
\underset{\sim}{H} \underset{\sim}{U}=\underset{\sim}{G} \underset{\sim}{P}+\underset{\sim}{B}
$$

Para evitar singularidades na determinação de (3.84), o ponto fonte " $\chi$ " no contorno, é retirado para fora do domínio, a uma distância do contorno dada por:

$$
d=\alpha l_{m}
$$

onde " $\alpha$ " é um coeficiente positivo $e$ " $l_{m}$ " é o comprimento médio dos elementos adjacentes, caso o ponto " $\chi$ " seja extremo, ou o próprio comprimento do elemento, caso " $\chi$ " coincida com o ponto intermediário " $k_{2}$ " (Fig. 3.8). 


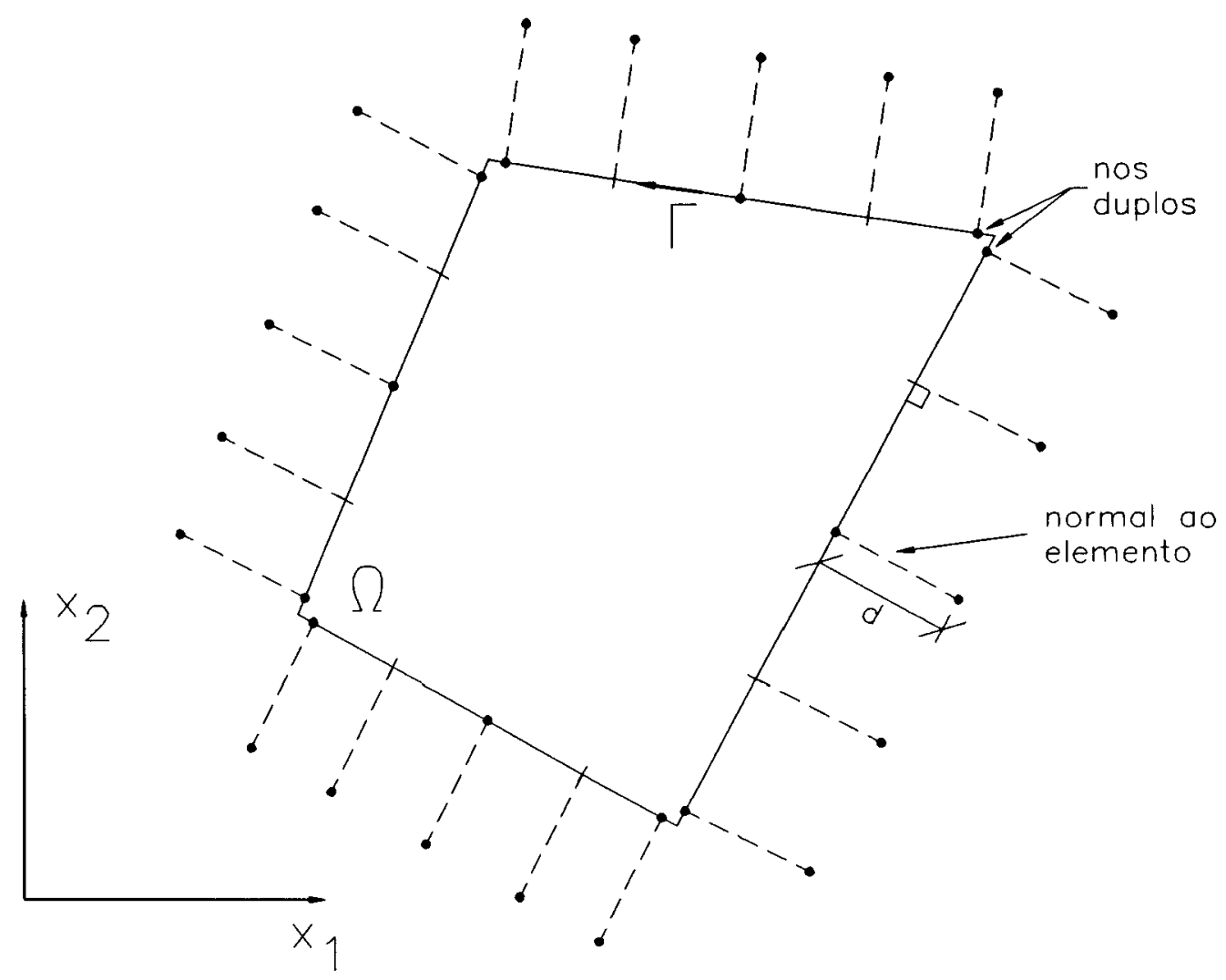

Fig. 3.8 - Colocação dos pontos fonte fora do domínio.

Quanto menor o valor de "d", mais precisos serão os resultados, desde que sejam tomadas precauções para evitarem-se erros de integração. As equações (3.84) são integradas numericamente usando-se quadratura de Gauss, devendo-se quando o ponto se aproximar do contorno, aumentarse o número dos pontos de integração, visando-se evitar os erros de integração. Segundo [140] os melhores resultados são obtidos para " $\alpha=0,25$ " usando-se 12 pontos de integração.

Observou-se nesse trabalho no entanto, que para valores muito pequenos de "d" os resultados são ruins, mesmo aumentando-se o número dos pontos de integração. Esse problema pode ser contornado dividindo-se o elemento que contenha o nó singular, em sub-elementos. Na Fig. 3.9, o nó $k_{1}$ (singular) foi retirado do contorno para uma nova posição $k_{1}^{\prime}$, a uma distância " $d=\alpha l$ ". O primeiro sub-elemento a partir de $k_{1}$ terá 0 
comprimento " $\alpha l$ " e sua extremidade estará a uma distância "R," de $k_{1}{ }_{1} .0$ segundo elemento terá um comprimento " $R_{1}$ " e sua extremidade estará a " $R_{2}$ " de $k_{1}$. O processo se repete até que a extremidade do último subelemento ultrapasse $o$ nó $k_{3}$, ficando este último sub-elemento com 0 comprimento igual a diferença entre o comprimento total do elemento e a soma dos comprimentos dos sub-elementos anteriores, conforme mostrado na Fig. 3.9.

Com esta técnica pode-se chegar a bons resultados mesmo para " $\alpha$ " abaixo de 0,001 . No entanto o valor adotado nesse trabalho é " $\alpha=0,01$ ",quando se usa sub-elementos, adotando-se 4 pontos de integração.

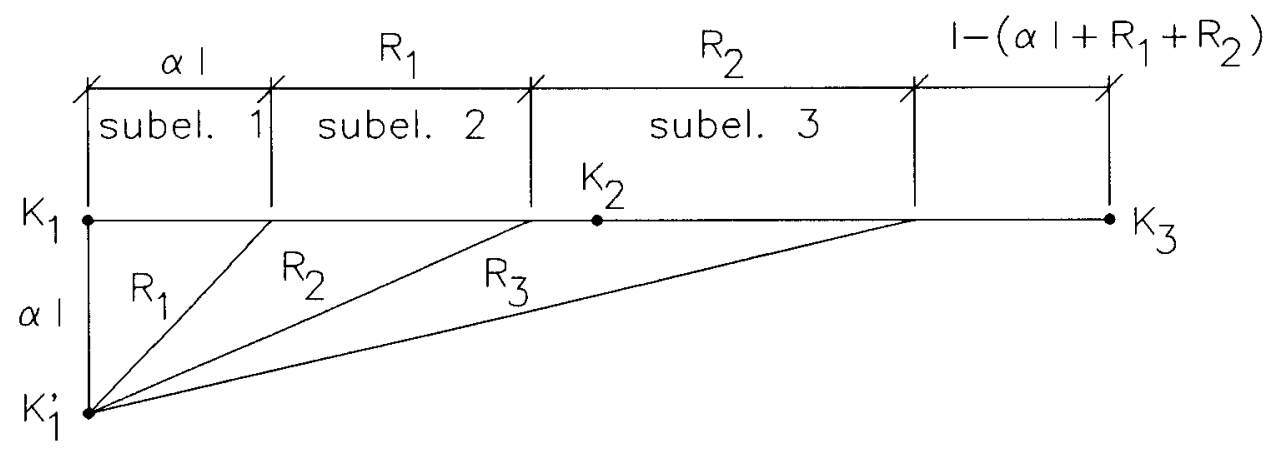

Fig. 3.9 - Divisão do elemento em sub-elementos.

Com a colocação do ponto fonte fora do domínio, a matriz " $C$ " em (3.85) torna-se nula.

Resolvidas as singularidades envolvidas na determinação das integrais (3.84), existem outras que se referem à descontinuidade da normal ao contorno, e à mudança nas condições de vinculação da placa. A primeira ocorre nos pontos angulosos ou cantos da placa, a segunda pode ocorrer 
mesmo quando há continuidade da normal ao contorno. Dentre as alternativas possiveis, opta-se neste trabalho pela adoção de nós duplos (Fig. 3.8), para representar tais descontinuidades. Estes nós duplos, assim denominados por possuírem as mesmas coordenadas, tornam independentes os valores nodais, antes e depois do ponto considerado.

\subsection{3 - INFLUÊNCIA DO CARREGAMENTO DE DOMÍNIO NO CÁLCULO DOS VALORES DE CONTORNO.}

A contribuiçăo da carga distribuída " $q(x)$ " na determinação dos valores de contorno é obtida integrando-se a equaçăo (3.32) para " $q(x)^{n}$ constante, ou as equações (3.44), caso " $q(x)$ " tenha variação linear. Em ambos os casos, a integração é feita sobre o contorno da região carregada " $\Gamma_{q}$. Analogamente ao que foi feito para o contorno da placa, o contorno da carga será dividido em "NECC" elementos, conforme ilustrado na Fig. 3.9.

As equações (3.32) e (3.44) para o contorno " $\Gamma_{q}$ " discretizado, podem ser escritas respectivamente como:

$$
\begin{aligned}
& q(\mathrm{x})=\text { constante } \\
& I_{i}=q(x) \sum_{j=1}^{N E C C} \int_{\Gamma_{q j}}\left[\mathrm{~V}_{i \alpha, \alpha}^{*}(\xi, x)-\frac{v}{(1-v) \lambda^{2}} u_{i \alpha}^{*}(\xi, x)\right] n_{\alpha} d \Gamma_{q_{j}}(x)
\end{aligned}
$$




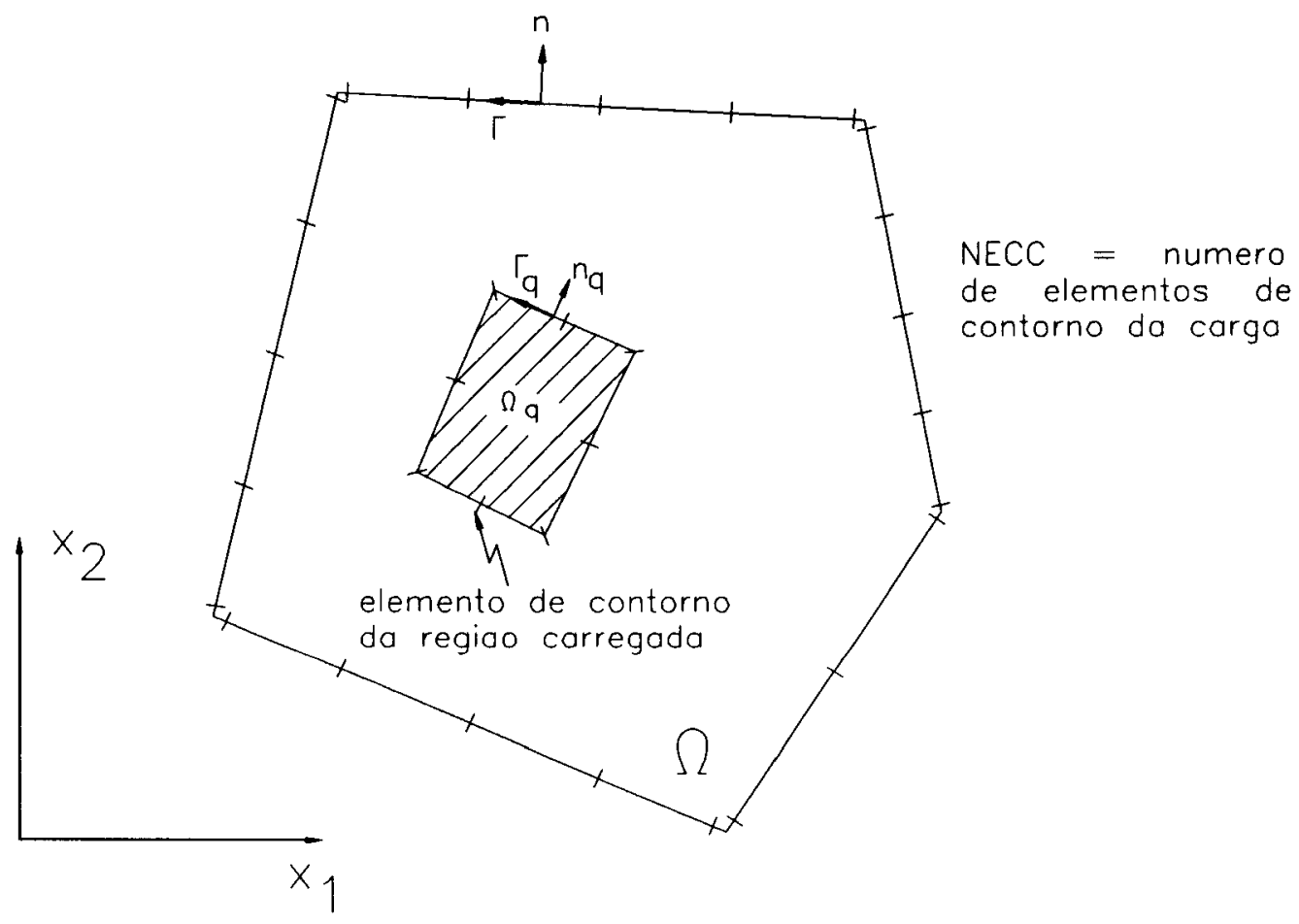

Fig. 3.10 -Discretização do contorno da região carregada " $\Omega_{q}$ "

$$
\begin{gathered}
q(x)=\text { linear } \\
I_{\alpha}=\frac{1}{96 \pi D} \sum_{j=1}^{N E C C}\left\{4 q(\xi) \int_{\Gamma_{q_{j}}}\left[R^{2}\left(2 \ln z-\frac{5}{3}\right)+\frac{12 v}{(1-v) \lambda^{2}}\right] r_{, \alpha} \cos \beta d \Gamma_{q j}(x)+\right. \\
\left.3 \int_{\Gamma_{q j}}\left(A_{1} \cos \theta+B_{1} \operatorname{sen} \theta\right)\left[R^{3}\left(2 \ln z-\frac{3}{2}\right)+\frac{8 v}{(1-v) \lambda^{2}} R\right] r_{, \alpha} \cos \beta d \Gamma_{q,}(x)\right\} \\
I_{3}=\frac{1}{8 \pi D(1-v) \lambda^{2}} \sum_{j=1}^{N E C C}\left\{q(\xi) \int_{\Gamma_{q j}} R\left[\frac{(1-v)}{4} z^{2}\left(\ln z-\frac{5}{4}\right)+2(v-2)\left(\ln z-\frac{1}{2}\right)\right]\right. \\
\cos \beta d \Gamma_{q j}(x)+\int_{\Gamma_{q j}}\left(A_{1} \cos \theta+B_{1} \operatorname{sen} \theta\right) R^{2}\left[\frac{(1-v)}{5} z^{2}\left(\ln z-\frac{5}{6}\right)+\frac{4}{3}(v-2)\left(\ln z-\frac{1}{3}\right)\right] \\
\left.\cos \beta d \Gamma_{q_{j}}(x)\right\}
\end{gathered}
$$


A influência da carga " $q(x)$ " na representação integral dos deslocamentos é obtida integrando-se (3.88) ou (3.89), dependendo da natureza do carregamento, para todos os "NECC" elementos do contorno " $\Gamma_{q}$ ", em relação a cada ponto de colocação " $\chi$. Como estes pontos foram levados para fora do domínio, eliminam-se desta forma, todas as singularidades das integrais acima, mesmo quando o contorno " $\Gamma_{\mathrm{q}}$ " da área carregada coincidir com o contorno da placa. A integração é efetuada numericamente através da quadratura de Gauss, e os valores das integrais são endereçados e armazenados convenientemente, caso haja mais de uma região carregada, no vetor " $B$ ".

A contribuição de cargas concentradas " $\mathrm{P}$ " no domínio, no cálculo dos valores de contorno, é feita diretamente com a equação (3.48) e os valores encontrados serăo armazenados para cada ponto do contorno " $\chi$ ", no vetor " $B$ ". Aqui mais uma vez não haverá singularidade, pelo mesmo motivo acima referido.

Caso a carga seja distribuída numa linha, a sua contribuição para o cálculo dos valores de contorno, é obtida integrando-se a equação (3.50) no contorno " $\Gamma_{q}$ ", que será também discretizado em elementos retos " $\Gamma_{q_{j}}$ ", onde os valores de " $q_{L}(x)$ " serão aproximados por funçōes do segundo grau. Com isto cada elemento " $\Gamma_{q_{j}}$ " necessitará de três pontos, dois extremos e um terceiro escolhido no meio do elemento. Os valores de " $q_{L}(x)$ " sobre 0 elemento "j" será dado por:

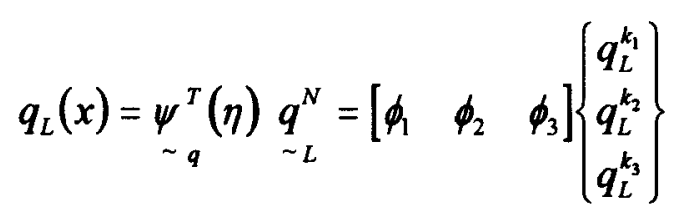




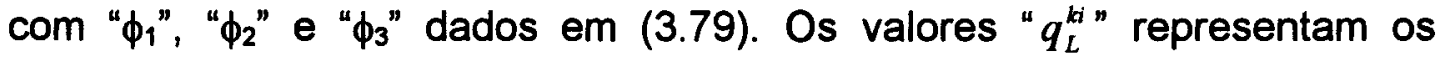
valores nodais da carga " $\mathrm{q}_{\mathrm{L}}(\mathrm{x})^{n}$.

Levando-se (3.90) em (3.50) e efetuando-se a mudança de variável de " $\Gamma_{q}$ " para " $\eta$ " obtém-se:

$$
I_{i}=\sum_{j=1}^{N E L C} \frac{L_{j}}{2} \int_{-1}^{+1}\left[u_{i 3}^{*}(\xi, x)-\frac{v}{(1-v) \lambda^{2}} u_{i \alpha, \alpha}^{*}(\xi, x)\right] \underset{\sim q}{\psi_{q}^{T}} d \eta(x) \underset{\sim L}{q_{L}^{N}}
$$

onde "NELC" é o número de elementos da linha de carga.

Integrando-se numericamente (3.91) para todos os "NELC" elementos da linha de carga, em relação a todos os pontos " $\chi$ " do contorno, multiplicando-se pelo vetor " $\underset{\sim L}{q^{N}}$ c correspondente, obtém-se a contribuição da linha de carga no cálculo dos valores de contorno, que serão endereçados e armazenados no vetor " $B$ ". Não haverá aqui também singularidade em (3.91), pelos mesmos motivos anteriores.

A contribuição dos momentos distribuídos em linha no cálculo dos valores incógnitos do contorno, é obtida de maneira análoga ao da carga distribuída, dividindo-se a linha de carga " $\Gamma_{m}$ " em elementos. Admitindo-se aproximação quadrática para " $m_{\alpha}^{L}(x)$ " sobre o elemento de contorno " $\Gamma_{m_{j}}$ ", pode-se escrever:

$$
m_{\alpha}^{L}(x)=\left\{\begin{array}{l}
m_{1}^{L}(x) \\
m_{2}^{L}(x)
\end{array}\right\}=\psi_{m}^{T}(\eta) m_{\alpha}^{N}=\left[\begin{array}{cccccc}
\phi_{1} & 0 & \phi_{2} & 0 & \phi_{3} & 0 \\
0 & \phi_{1} & 0 & \phi_{2} & 0 & \phi_{3}
\end{array}\right]\left\{\begin{array}{l}
m_{1}^{k_{1}} \\
m_{2}^{k_{1}} \\
m_{1}^{k_{2}} \\
m_{2}^{k_{2}} \\
m_{1}^{k_{3}} \\
m_{2}^{k_{3}}
\end{array}\right\}
$$


com " $\phi_{1}$ ", " $\phi_{2}$ " e " $\phi_{3}$ " dados em (3.79). Os valores " $m_{\alpha}^{k i n}$ representam os valores nodais dos momentos distribuídos " $m_{\alpha}$ ". A partir da discretização do contorno " $\Gamma_{m}$ " e da expressão de " $m_{\alpha}^{L}(x)$ " dada em (3.92) pode-se escrever (3.64) como:

$$
\int_{\Gamma_{m}} m_{\alpha}^{L}(x) u_{i \alpha}^{*}(\xi, x) d \Gamma_{m}(x)=\sum_{j=1}^{N E L C} \frac{L_{j}}{2} \int_{-1}^{+1} u_{i \alpha}^{*}(\xi, x) \underset{\sim m}{\psi_{\sim}^{T}}(\eta) d \eta(x) \underset{\sim \alpha}{m_{\alpha}^{N}}
$$

Integrando-se numericamente (3.93) para cada elemento "j" da linha de momentos, em relação a todos os pontos nodais do contorno e multiplicando-se pelo vetor nodal correspondente " $m_{\alpha}^{k}$ ", obtém-se a contribuição da linha de momentos no cálculo dos valores de contorno.

A contribuição em cada ponto do contorno é endereçada e armazenada convenientemente no vetor " $B^{\text {" }}$ da equação (3.85). Deve-se salientar que aqui também não haverá singularidades.

\subsection{4 - DESLOCAMENTOS NOS PONTOS INTERNOS.}

Os deslocamentos em pontos internos são expressos em (3.11) e poderão ser obtidos diretamente a partir do conhecimento dos valores incógnitos no contorno. Isto traduz uma vantagem do Método dos Elementos de Contorno: a determinação de valores internos não acarreta aumento no número de equaçōes do sistema. De maneira análoga, a equação (3.11) será discretizada em funçăo da divisão do contorno da placa em "NECP" elementos. Assim, a equação (3.11) discretizada é expressa por: 


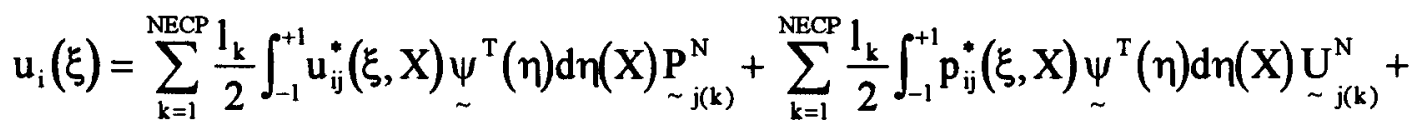

$$
\begin{aligned}
& \int_{\Omega_{q}} q(x)\left[u_{i 3}^{*}(\xi, x)-\frac{v}{(1-v) \lambda^{2}} u_{i \alpha, \alpha}^{*}(\xi, x)\right] d \Omega_{q}(x)+\int_{\Omega_{m}} m_{\alpha}(x) u_{i \alpha}^{*}(\xi, x) d \Omega_{m}(x) \quad(3.94)
\end{aligned}
$$

Escrevendo-se a equação (3.94) para os "NPI" pontos internos, onde haja interesse no cálculo dos deslocamentos, obtém-se:

$$
\underset{\sim}{U_{i}}=\underset{\sim}{G_{i}} \underset{\sim}{P_{\Gamma}}-\underset{\sim}{H_{i}} U_{\Gamma}+B_{\sim}
$$

onde, " $U_{i}$ " é o vetor dos deslocamentos nos pontos internos. As matrizes " $G_{i}$ " e " $H_{i}$ ", para pontos internos, são obtidas de forma análoga à obtenção de " $G^{n}$ e " $H^{\text {" }}$, para pontos do contorno " $\Gamma$ ". O vetor " $B_{i}$ " contém a influência dos carregamentos de domínio na determinação dos deslocamentos em pontos internos e sua determinação se faz de maneira análoga à obtida para " $B$ ". " $P_{\Gamma}$ " e " $U_{\Gamma}$ " são os vetores dos deslocamentos e forças de superfície para os pontos nodais, do contorno " $\Gamma$ ".

Os deslocamentos nos pontos internos são calculados em relação ao sistema de referência global " $x_{1}{ }^{n}, "{ }_{2}{ }^{n}$ e " $x_{3}{ }^{n}$. Assim para um ponto interno qualquer " $k$ " tem-se:

$$
\underset{\sim i}{U_{i}^{k}}=\left\{\begin{array}{c}
\phi_{x_{1}}^{k} \\
\phi_{x_{2}}^{k} \\
w^{k}
\end{array}\right\}
$$

Na equaçăo (3.95) os coeficientes de " $G_{i}$ " e " $\mathrm{H}_{i}$ " são calculados numericamente, sem ocorrência de singularidades, uma vez que $\xi$ é interno 
e X está no contorno. Já com os termos de " $B_{i}{ }^{n}$ surgirão singularidades quando o ponto interno " $\xi$ ", para o qual se escrevem as equações dos deslocamentos, coincidir com a linha de carga, linha de momentos ou com a carga concentrada " $\mathrm{P}$ ".

Para o caso da carga concentrada " $\mathrm{P}$ ", quando o ponto " $\xi$ " coincidir com o ponto de aplicação da carga, a contribuiçăo para os coeficientes " $B_{i}$ " na equação (3.95) é obtida através da expressão (3.48). Analisando-se as soluções fundamentais envolvidas nesta expressão, dadas em (2.32) e (3.21), vê-se que na situação limite, quando " $r$ " tende a zero, pode-se escrever (3.48) como:

$$
\lim _{r \rightarrow 0} P\left[u_{i 3}^{*}(\xi, x)-\frac{v}{(1-v) \lambda^{2}} u_{i \alpha, \alpha}^{*}(\xi, x)\right]=0
$$

Para o caso de cargas e momentos distribuídos em linha, seja a Fig.3.11, onde está representado um elemento " $k$ " qualquer da linha de carga " $\Gamma_{q}$ " ou " $\Gamma_{m}$ ", mostrando-se os três pontos nodais " $k_{1}$ ", " $k_{2}{ }^{\text {" } e ~ " ~} k_{3}$ ". Nesta figura o ponto " $\xi$ " coincide com o ponto nodal " $k_{2}$ " $\left(\xi=k_{2}\right)$.

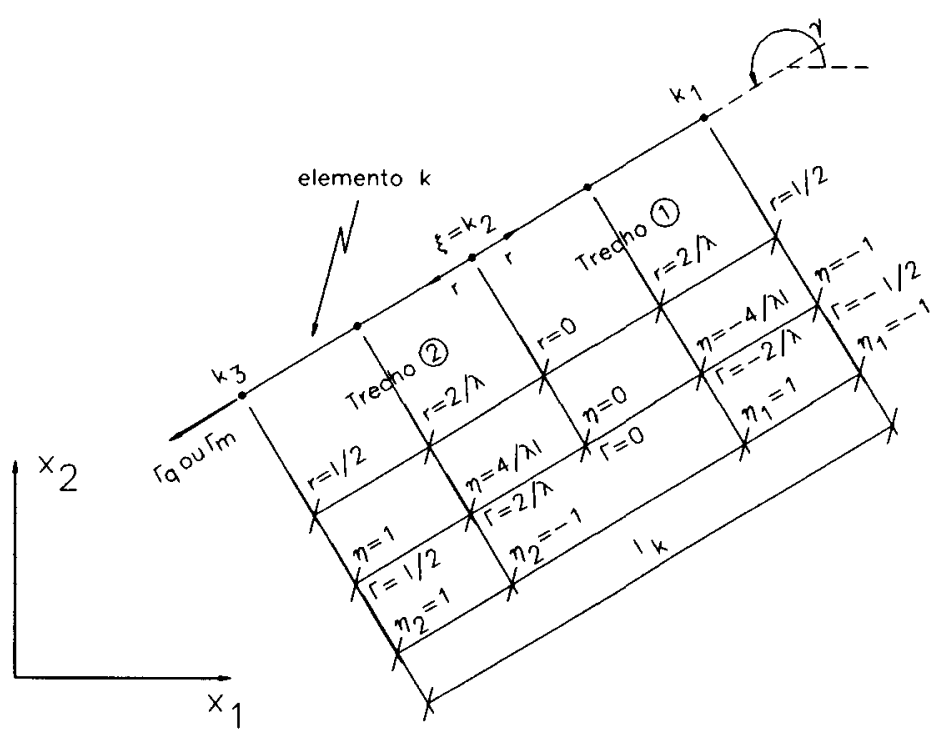

Fig. 3.11 - Elemento de carga " $\Gamma_{q}$ " ou " $\Gamma_{m}{ }^{n}$ com " $\xi=k 2$ ". 
Analisando-se a Fig. 3.11 pode-se escrever:

\section{Trecho 1}

$$
\begin{gathered}
r=-\Gamma=-\frac{l}{2} \eta \\
d r=-d \Gamma=-\frac{l}{2} d \eta
\end{gathered}
$$

Trecho 2

$$
\begin{gathered}
r=\Gamma=\frac{l}{2} \eta \\
d r=d \Gamma=\frac{l}{2} d \eta
\end{gathered}
$$

A contribuição da linha de carga " $q_{L}(x)$ " na determinação dos deslocamentos internos é dado na expressão (3.91), que será analisada agora para o elemento " $k$ ", quando " $\xi=k 2$ ". Assim pode-se escrever (3.91): obs: serăo retirados os indices $(x)$ e $(\xi, x)$, por simplificaçăo.

$$
\begin{aligned}
& \int_{\Gamma_{q}} q_{L}\left[u_{i 3}^{*}-\frac{v}{(1-v) \lambda^{2}} u_{i \alpha, \alpha}^{*}\right] d \Gamma_{q}=\lim _{\varepsilon \rightarrow 0} \int_{\Gamma_{s}} q_{L}\left[u_{i 3}^{*}-\frac{v}{(1-v) \lambda^{2}} u_{i \alpha, \alpha}^{*}\right] d \Gamma_{\varepsilon}+ \\
& \lim _{s \rightarrow 0} \int_{\Gamma_{\Gamma_{q}} \Gamma_{s}} q_{L}\left[u_{i 3}^{*}-\frac{v}{(1-v) \lambda^{2}} u_{i a, \alpha}^{*}\right] d \Gamma_{q}
\end{aligned}
$$

Na expressão (3.99) o contorno " $\Gamma_{\varepsilon}$ " é o mesmo da Fig. 3.5. O primeiro limite do segundo membro de (3.99) se anula, e " $\left(\Gamma_{q}-\bar{\Gamma}_{\varepsilon}\right)$ " tende a " $\Gamma_{q}$ " quando " $\varepsilon$ " tende a zero. Analisando-se a Fig. 3.11 pode-se escrever (3.99) como: 


$$
\begin{aligned}
& \int_{\Gamma_{q}} q_{L}\left[u_{i 3}^{*}-\frac{v}{(1-v) \lambda^{2}} u_{i \alpha, \alpha}^{*}\right] d \Gamma_{q}=\int_{-1 / 2}^{0} q_{L}\left[u_{i 3}^{*}-\frac{v}{(1-v) \lambda^{2}} u_{i \alpha}^{*}\right] d \Gamma_{q_{1}}+ \\
& \int_{0}^{l / 2} q_{L}\left[u_{i 3}^{*}-\frac{v}{(1-v) \lambda^{2}} u_{i \alpha}^{*}\right] d \Gamma_{q_{2}}
\end{aligned}
$$

onde a primeira parcela do segundo membro de (3.100) representa a integração no trecho 1 do elemento "k" (Fig. 3.10), e a segunda no trecho 2.

Mudando-se a variável de " $\Gamma_{q}$ " para " $r$ " em (3.100) e observando-se (3.98) para cada trecho do elemento " $k$ ", a expressão (3.100) pode ser reescrita como:

$$
\begin{aligned}
& \int_{\Gamma_{q}} q_{L}\left[u_{i 3}^{*}-\frac{v}{(1-v) \lambda^{2}} u_{i \alpha, \alpha}^{*}\right] d \Gamma_{q}=\int_{0}^{l / 2} q_{L}\left[u_{i 3}^{*}-\frac{v}{(1-v) \lambda^{2}} u_{i \alpha}^{*}\right] d r_{1}+ \\
& \int_{0}^{l / 2} q_{L}\left[u_{i 3}^{*}-\frac{v}{(1-v) \lambda^{2}} u_{i \alpha}^{*}\right] d r_{2}
\end{aligned}
$$

Substituindo-se o valor de "qL", dado em (3.90) na expressão (3.101), obtém-se:

$$
\begin{aligned}
& \int_{\Gamma_{q}} q_{L}\left[u_{i 3}^{*}-\frac{v}{(1-v) \lambda^{2}} u_{i \alpha, \alpha}^{*}\right] d \Gamma_{q}=\int_{0}^{l / 2}\left[u_{i 3}^{*}-\frac{v}{(1-v) \lambda^{2}} u_{i \alpha, \alpha}^{*}\right] \underset{\sim}{\psi^{T}}(\eta) d r_{1} \underset{\sim}{q_{L}^{N}}+ \\
& \int_{0}^{l / 2}\left[u_{i 3}^{*}-\frac{v}{(1-v) \lambda^{2}} u_{i \alpha, \alpha}^{*}\right] \underset{\sim}{\psi^{T}}(\eta) d r_{2} \underset{\sim L}{q_{L}^{N}}
\end{aligned}
$$

O vetor " $\psi^{T}$ ", conforme os valores de suas componentes " $\phi_{1}$ ", " $\phi_{2}$ " e " $\phi_{3}$ ", expressos em (3.79), é função da variável homogênea " $\eta$ ". Neste caso, para " $\xi=k_{2}$ ", o elemento é dividido em dois trechos, com valores distintos para " $r$ ", ficando as fórmulas (3.79) transformadas em: 
Trecho 1

$$
\begin{aligned}
& \eta=-\frac{2}{l} r \\
& \phi_{1}(r)=\frac{1}{2} \eta(\eta-1)=\left(\frac{2 r^{2}}{l^{2}}+\frac{r}{l}\right) \\
& \phi_{2}(r)=(1+\eta)(1-\eta)=\left(1-\frac{4 r^{2}}{l^{2}}\right) \\
& \phi_{3}(r)=\frac{1}{2} \eta(\eta+1)=\left(\frac{2 r^{2}}{l^{2}}-\frac{r}{l}\right)
\end{aligned}
$$

\section{Trecho 2}

$$
\begin{gathered}
\eta=\frac{2}{l} r \\
\phi_{1}(r)=\frac{1}{2} \eta(\eta-1)=\left(\frac{2 r^{2}}{l^{2}}-\frac{r}{l}\right) \\
\phi_{2}(r)=(1+\eta)(1-\eta)=\left(1-\frac{4 r^{2}}{l^{2}}\right) \\
\phi_{3}(r)=\frac{1}{2} \eta(\eta+1)=\left(\frac{2 r^{2}}{l^{2}}+\frac{r}{l}\right)
\end{gathered}
$$

A partir das equações (2.32), (3.21), (3.103) e (3.104), sabendo-se que: 
94

$$
r_{, \alpha}=r_{, \alpha_{2}}=-r_{, \alpha_{1}}=\left\{\begin{array}{l}
\cos \gamma \\
\operatorname{sen} \gamma
\end{array}\right.
$$

onde " $\gamma$ " é o ângulo que o elemento " $\Gamma_{q}$ " ou " $\Gamma_{m}$ " faz com o eixo " $x_{1}$ ", pode-se escrever (3.102) como:

para $\mathrm{i} \leq 2$

$$
\begin{aligned}
& \int_{\Gamma_{q}} q_{L}\left[u_{\alpha 3}^{*}-\frac{v}{(1-v) \lambda^{2}} u_{\alpha \beta, \beta}^{*}\right] d \Gamma_{q}=\frac{r_{a}}{8 \pi D}\left\{\left[\frac{4}{l} \int_{0}^{l / 2} r^{2} \ln z d r-\frac{l^{2}}{12}+\frac{4 v}{(1-v) \lambda^{2}}\right] q^{k_{1}}+\right. \\
& \left.\left[-\frac{4}{l} \int_{0}^{l / 2} r^{2} \ln z d r+\frac{l^{2}}{12}-\frac{4 v}{(1-v) \lambda^{2}}\right] q^{k_{3}}\right\}
\end{aligned}
$$

para $i=3$

$$
\begin{gathered}
\int_{\Gamma_{q}} q_{L}\left[u_{33}^{*}-\frac{v}{(1-v) \lambda^{2}} u_{3 \alpha, a}^{*}\right] d \Gamma_{q}=\frac{1}{4 \pi D}\left\{\left[\frac{2}{l^{2}} \int_{0}^{l / 2} r^{4} \ln z d r-\frac{l^{3}}{80}+\frac{1}{(1-v) \lambda^{2} l^{2}}\right.\right. \\
\left.\left(-16 \int_{0}^{l / 2} r^{2} \ln z d r+8 v \int_{0}^{l / 2} r^{2} \ln z d r\right)\right] q^{k_{1}}+\left[\int_{0}^{l / 2} r^{2} \ln z d r-\frac{4}{l^{2}} \int_{0}^{l / 2} r^{4} \ln z d r-\frac{l^{3}}{60}+\right. \\
\left.\frac{1}{(1-v) \lambda^{2}}\left(-8 \int_{0}^{l / 2} \ln z d r+\frac{32}{l^{2}} \int_{0}^{l / 2} r^{2} \ln z d r+4 v \int_{0}^{l / 2} \ln z d r-\frac{16 v}{l^{2}} \int_{0}^{l / 2} r^{2} \ln z d r\right)\right] q^{k_{2}}+ \\
\left.\left[\frac{2}{l^{2}} \int_{0}^{l / 2} r^{4} \ln z d r-\frac{l^{3}}{80}+\frac{1}{(1-v) \lambda^{2} l^{2}}\left(-16 \int_{0}^{l / 2} r^{2} \ln z d r+8 v \int_{0}^{l / 2} r^{2} \ln z d r\right)\right] q^{k_{3}}\right\}
\end{gathered}
$$

onde " $q_{1}^{k}$ ", " $q_{2}^{k}{ }^{\prime} e^{~ " ~} q_{3}^{k}$ " são os valores nodais para a carga distribuída " $q_{L}$ ". As integrais em "In z" que aparecem em (3.107) são dadas por:

$$
\int_{0}^{a} r^{n} \ln z d r=\frac{a^{n+1}}{n+1}\left(\ln \lambda a-\frac{1}{n+1}\right), 0 \leq n \leq 4
$$


Conhecendo-se os valores nodais para a carga " $q_{L}\left(q_{1}^{k}, q_{2,}^{k}{ }_{k 3}\right)^{n}$ determinam-se as contribuições da carga distribuida para a formação de " $B_{i}$ ". As duas primeiras, correspondentes às rotações " $\phi_{x 1}{ }^{n}$ e " $\phi_{x}$ ", são obtidas através da expressão (3.106) e a terceira, correspondente ao deslocamento transversal " $w$ ", é obtida com a expressão (3.107). Estas contribuições para os deslocamentos em pontos internos foram deduzidas quando o ponto fonte " $\xi$ ", coincidiu com o ponto nodal do elemento " $k_{2}$ " (Fig.3.11).

Será deduzida, a partir da Fig. 3.12, a contribuiçăo da carga distribuída " $\mathrm{L}_{L}$ " para o cálculo dos deslocamentos em pontos internos, quando o ponto fonte " $\xi$ ", coincide com o ponto nodal " $k_{1}$ " $\left(\xi=k_{1}\right)$.

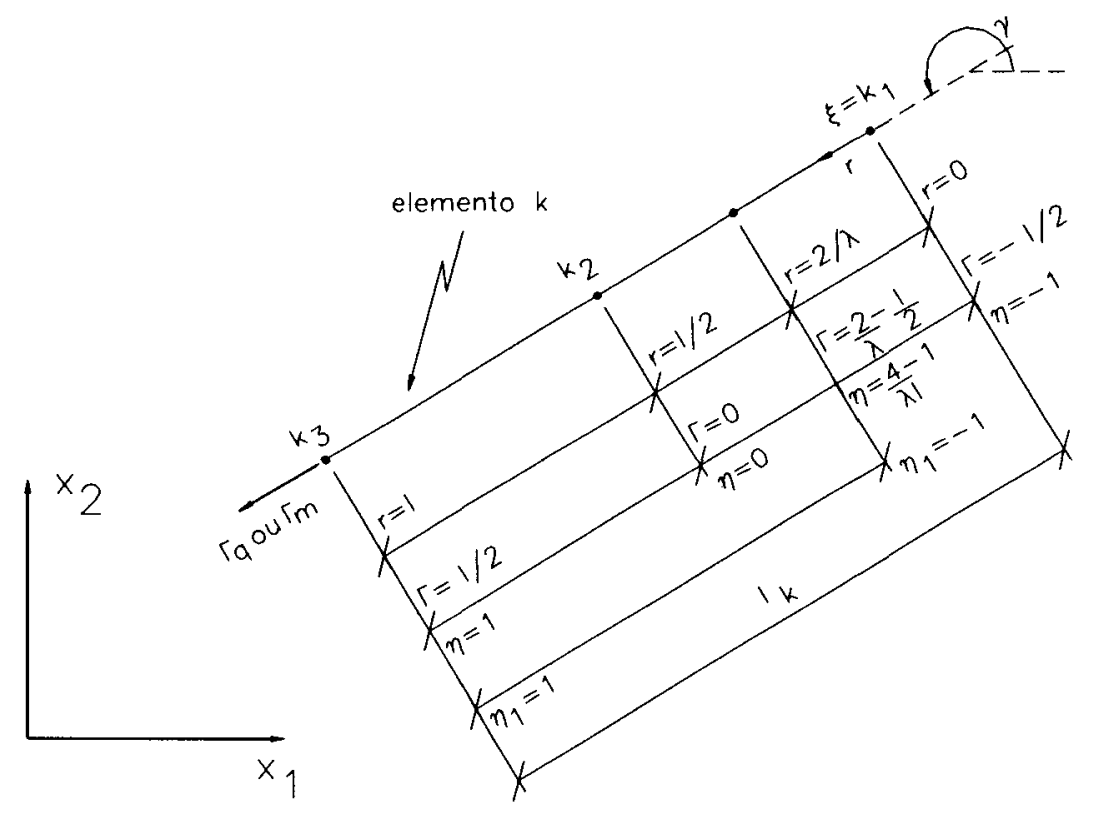

Fig. 3.12 - Elemento de carga " $\Gamma_{q}$ " ou " $\Gamma_{m}$ " com " $\xi=k_{1}$ ".

Da Fig. 3.12 pode-se escrever:

$$
r=\Gamma+\frac{l}{2}=\frac{l}{2}(\eta+1)
$$




$$
d r=d \Gamma=\frac{l}{2} d \eta
$$

Analogamente ao que foi desenvolvido quando " $\xi=k_{2}$ ", pode-se escrever a expressão (3.100) para o caso presente como:

$$
\int_{\Gamma_{q}} q_{L}\left[u_{i 3}^{*}-\frac{v}{(1-v) \lambda^{2}} u_{i \alpha, \alpha}^{*}\right] d \Gamma_{q}=\int_{0}^{l} q_{L}\left[u_{i 3}^{*}-\frac{v}{(1-v) \lambda^{2}} u_{i \alpha, \alpha}^{*}\right] d r
$$

Considerando-se (3.109), as expressões (3.103) e (3.104) são agora escritas como:

$$
\begin{aligned}
& \eta=\frac{2}{l} r-1 \\
& \phi_{1}(r)=\frac{1}{2} \eta(\eta-1)=\left(\frac{2 r^{2}}{l^{2}}-\frac{3 r}{l}+1\right) \\
& \phi_{2}(r)=(1+\eta)(1-\eta)=\left(\frac{4 r}{l}-\frac{4 r^{2}}{l^{2}}\right) \\
& \phi_{3}(r)=\frac{1}{2} \eta(\eta+1)=\left(\frac{2 r^{2}}{l^{2}}-\frac{r}{l}\right)
\end{aligned}
$$

A partir de (3.111) as expressões que dão a influência da carga " $q_{L}$ ", quando " $\xi=k_{1}$ ", no cálculo dos deslocamentos internos, são dadas por: 
97

para $i \leq 2$

$$
\begin{aligned}
& \int_{\Gamma_{q}} q_{L}\left[u_{\alpha 3}^{*}-\frac{v}{(1-v) \lambda^{2}} u_{\alpha \beta, \beta}^{*}\right] d \Gamma_{q}=\frac{r_{, \alpha}}{8 \pi D}\left\{\left[\frac{4}{l^{2}} \int_{0}^{l} r^{3} \ln z d r-\frac{6}{l} \int_{0}^{l} r^{2} \ln z d r+\right.\right. \\
& \left.2 \int_{0}^{l} r \ln z d r+\frac{4 v}{(1-v) \lambda^{2}}\left(-2+\ln l-\lim _{s \rightarrow 0} \ln \varepsilon\right)\right] q^{k_{1}}+ \\
& {\left[\frac{8}{l} \int_{0}^{l} r^{2} \ln z d r-\frac{8}{l^{2}} \int_{0}^{l} r^{3} \ln z d r-\frac{l^{2}}{3}+\frac{8 v}{(1-v) \lambda^{2}}\right] q^{k_{2}}+} \\
& \left.\left[\frac{4}{l^{2}} \int_{0}^{l} r^{3} \ln z d r-\frac{2}{l} \int_{0}^{l} r^{2} \ln z d r-\frac{l^{2}}{6}\right] q^{k_{3}}\right\}
\end{aligned}
$$

para $i=3$

$$
\begin{gathered}
\int_{\Gamma_{q}} q_{L}\left[u_{33}^{*}-\frac{v}{(1-v) \lambda^{2}} u_{3 a, a}^{*}\right] d \Gamma_{q}=\frac{1}{8 \pi D}\left\{\left[\frac{2}{l^{2}} \int_{0}^{l} r^{4} \ln z d r-\frac{3}{l} \int_{0}^{l} r^{3} \ln z d r+\right.\right. \\
\int_{0}^{l} r^{2} \ln z d r+\frac{l^{3}}{60}+\frac{1}{(1-v) \lambda^{2}}\left(-\frac{16}{l^{2}} \int_{0}^{l} r^{2} \ln z d r+\frac{24}{l} \int_{0}^{l} r \ln z d r-\right. \\
\left.\left.8 \int_{0}^{l} \ln z d r+\frac{8 v}{l^{2}} \int_{0}^{l} r^{2} \ln z d r-\frac{12 v}{l^{2}} \int_{0}^{l} r \ln z d r+4 v \int_{0}^{l} \ln z d r\right)\right] q^{k_{1}}+ \\
{\left[\frac{4}{l} \int_{0}^{l} r^{3} \ln z d r-\frac{4}{l^{2}} \int_{0}^{l} r^{4} \ln z d r-\frac{l^{3}}{5}+\frac{1}{(1-v) \lambda^{2}}\left(-\frac{32}{l} \int_{0}^{l} r \ln z d r+\frac{32}{l^{2}} \int_{0}^{l} r^{2} \ln z d r+\right.\right.} \\
\left.\left.\frac{16 v}{l} \int_{0}^{l} r \ln z d r-\frac{16 v}{l^{2}} \int_{0}^{l} r^{2} \ln z d r\right)\right] q^{k_{2}}+\left[\frac{2}{l^{2}} \int_{0}^{l} r^{4} \ln z d r-\frac{1}{l} \int_{0}^{l} r^{3} \ln z d r-\frac{3 l^{3}}{20}+\right. \\
\left.\left.\frac{1}{(1-v) \lambda^{2}}\left(-\frac{16}{l^{2}} \int_{0}^{l} r^{2} \ln z d r+\frac{8}{l} \int_{0}^{l} r \ln z d r+\frac{8 v}{l^{2}} \int_{0}^{l} r^{2} \ln z d r-\frac{4 v}{l} \int_{0}^{l} r \ln z d r\right)\right] q^{k_{3}}\right\}
\end{gathered}
$$

onde " $r, \alpha$ " é o mesmo valor dado em (3.105) e as integrais envolvendo "In $z$ " são dadas em (3.108). 
Quando o ponto fonte " $\xi$ " coincide com o ponto " $k_{3}$ " do elemento de carga, situação ilustrada na Fig. 3.13, as expressões (3.109) ficam agora escritas como:

$$
\begin{aligned}
& r=\frac{l}{2}-\Gamma=\frac{l}{2}(1-\eta) \\
& d r=-d \Gamma=-\frac{l}{2} d \eta
\end{aligned}
$$

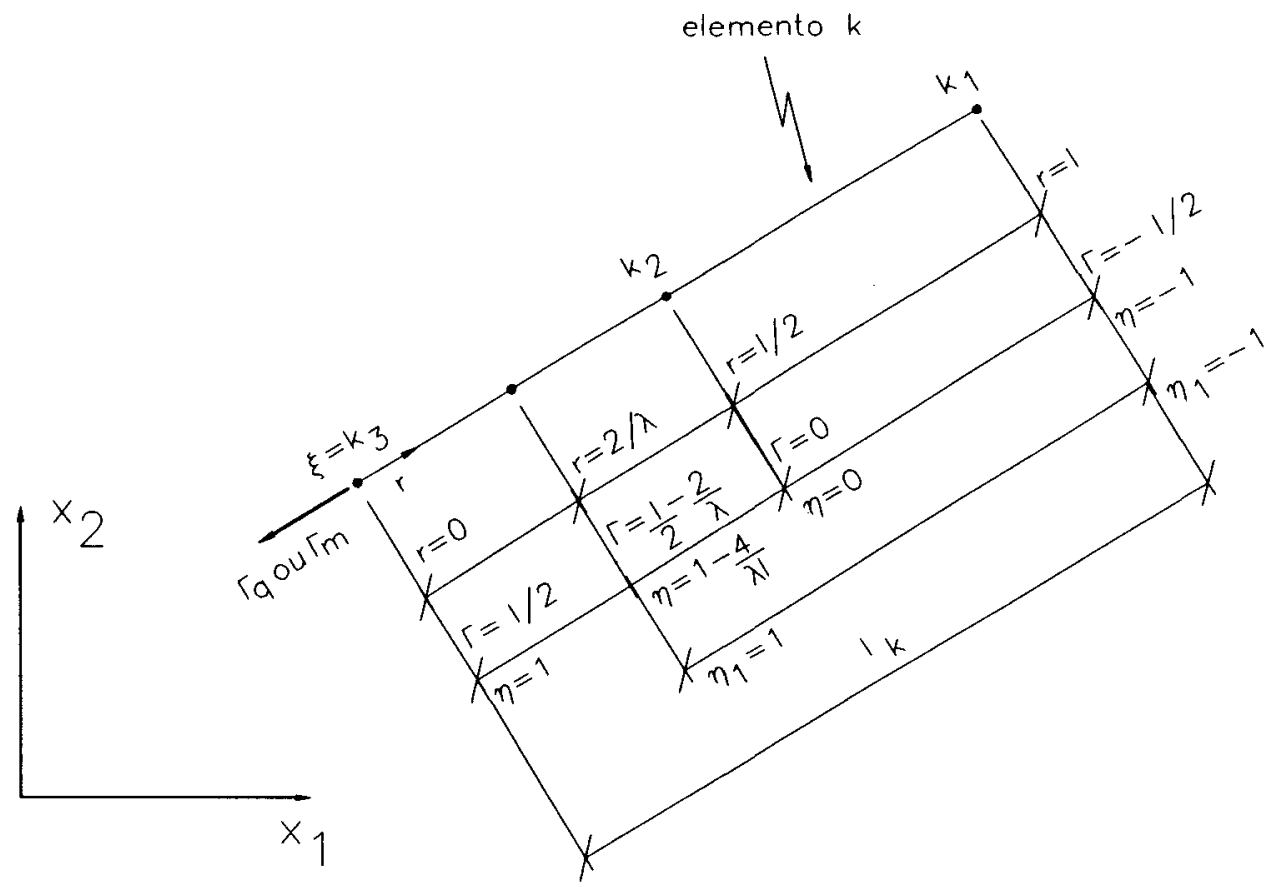

Fig. 3.13 - Elemento de carga " $\Gamma_{q}$ " ou " $\Gamma_{m}$ " com " $\xi=k_{3}$ "

Analogamente (3.111) fica agora:

$$
\begin{aligned}
& \eta=1-\frac{2}{l} r \\
& \phi_{1}(r)=\frac{1}{2} \eta(\eta-1)=\left(\frac{2 r^{2}}{l^{2}}-\frac{r}{l}\right)
\end{aligned}
$$




$$
\begin{aligned}
& \phi_{2}(r)=(1+\eta)(1-\eta)=\left(\frac{4 r}{l}-\frac{4 r^{2}}{l^{2}}\right) \\
& \phi_{3}(r)=\frac{1}{2} \eta(\eta+1)=\left(\frac{2 r^{2}}{l^{2}}-\frac{3 r}{l}+1\right)
\end{aligned}
$$

A partir de (3.115) a influência da carga distribuída " $q_{L}$ ", no cálculo dos deslocamentos internos, quando " $\xi=k_{3}{ }^{n}$ é dada por:

\section{para $\mathrm{i} \leq 2$}

$$
\begin{aligned}
& \int_{\Gamma_{q}} q_{L}\left[u_{\alpha 3}^{*}-\frac{v}{(1-v) \lambda^{2}} u_{\alpha \beta, \beta}^{*}\right] d \Gamma_{q}=\frac{r_{, a}}{8 \pi D}\left\{\left[\frac{4}{l^{2}} \int_{0}^{l} r^{3} \ln z d r-\frac{2}{l} \int_{0}^{l} r^{2} \ln z d r-\frac{l^{2}}{6}\right] q^{k_{1}}+\right. \\
& {\left[\frac{8}{l} \int_{0}^{l} r^{2} \ln z d r-\frac{8}{l^{2}} \int_{0}^{l} r^{3} \ln z d r-\frac{l^{2}}{3}+\frac{8 v}{(1-v) \lambda^{2}}\right] q^{k_{2}}+} \\
& \left.\left[\frac{4}{l^{2}} \int_{0}^{l} r^{3} \ln z d r-\frac{6}{l} \int_{0}^{l} r^{2} \ln z d r+2 \int_{0}^{l} r \ln z d r+\frac{4 v}{(1-v) \lambda^{2}}\left(-2+\ln l-\lim _{\varepsilon \rightarrow 0} \ln \varepsilon\right)\right] q^{k_{3}}\right\}
\end{aligned}
$$

para $\mathrm{i}=3$

$$
\begin{aligned}
& \int_{\Gamma_{q}} q_{L}\left[u_{33}^{*}-\frac{v}{(1-v) \lambda^{2}} u_{3 \alpha, \alpha}^{*}\right] d \Gamma_{q}=\frac{1}{8 \pi D}\left\{\left[\frac{2}{l^{2}} \int_{0}^{l} r^{4} \ln z d r-\frac{1}{l} \int_{0}^{l} r^{3} \ln z d r-\frac{3 l^{3}}{20}+\right.\right. \\
& \left.\frac{1}{(1-v) \lambda^{2}}\left(-\frac{16}{l^{2}} \int_{0}^{l} r^{2} \ln z d r+\frac{8}{l} \int_{0}^{l} r \ln z d r+\frac{8 v}{l^{2}} \int_{0}^{l} r^{2} \ln z d r-\frac{4 v}{l} \int_{0}^{l} r \ln z d r\right)\right] q^{k_{1}}+ \\
& {\left[\frac{4}{l} \int_{0}^{l} r^{3} \ln z d r-\frac{4}{l^{2}} \int_{0}^{l} r^{4} \ln z d r-\frac{l^{3}}{5}+\frac{1}{(1-v) \lambda^{2}}\left(-\frac{32}{l} \int_{0}^{l} r \ln z d r+\frac{32}{l^{2}} \int_{0}^{l} r^{2} \ln z d r+\right.\right.} \\
& \left.\left.\frac{16 v}{l} \int_{0}^{l} r \ln z d r-\frac{16 v}{l^{2}} \int_{0}^{l} r^{2} \ln z d r\right)\right] q^{k_{2}}+\left[\frac{2}{l^{2}} \int_{0}^{l} r^{4} \ln z d r-\frac{3}{l} \int_{0}^{l} r^{3} \ln z d r+\right. \\
& \int_{0}^{l} r^{2} \ln z d r+\frac{l^{3}}{60}+\frac{1}{(1-v) \lambda^{2}}\left(-\frac{16}{l^{2}} \int_{0}^{l} r^{2} \ln z d r+\frac{24}{l} \int_{0}^{l} r \ln z d r-8 \int_{0}^{l} \ln z d r+\right.
\end{aligned}
$$




$$
\left.\left.\frac{8 v}{l^{2}} \int_{0}^{l} r^{2} \ln z d r-\frac{12 v}{l} \int_{0}^{l} r \ln z d r+4 v \int_{0}^{l} \ln z d r\right)\right] q^{k_{3}}
$$

onde " $r_{, \alpha}$ " é dado agora por:

$$
r_{\alpha}=\left\{\begin{array}{l}
-\cos \gamma \\
-\operatorname{sen} \gamma
\end{array}\right.
$$

Admitindo-se a continuidade de " $q_{L}$ " e analisando-se (3.105) e (3.118), nota-se que os " $\lim _{\varepsilon \rightarrow 0} \ln \varepsilon$ " que aparecem nos coeficientes de " $q$ " (3.112) e de " $q^{\mathbf{k} 3 "}$ (3.117) se anulam, quando se consideram dois elementos de carga adjacentes.

Será analisada agora a contribuição da linha de momentos " $m_{\alpha}^{L}$ " no cálculo dos deslocamentos internos, quando o ponto fonte coincide com um dos pontos nodais do elemento analisado.

Será inicialmente considerada a situação em que o ponto fonte coincide com o ponto nodal " $k_{2}$ ", Fig. 3.11. A contribuiçăo das linhas de momentos no cálculo dos deslocamentos internos, é dada na expressão (3.93), que será analisada apenas para o elemento " $k$ ", que contém o nó singular " $\xi$ ". Assim (3.93) pode ser reescrita como:

$$
\int_{\Gamma_{m}} m_{\alpha}^{L} u_{i \alpha}^{*} d \Gamma_{m}=\lim _{\varepsilon \rightarrow 0} \int_{\Gamma_{s}} m_{\alpha}^{L} u_{i \alpha}^{*} d \Gamma_{\varepsilon}+\lim _{s \rightarrow 0} \int_{\Gamma_{m}-\bar{\Gamma}_{s}} m_{\alpha}^{L} u_{i \alpha}^{*} d \Gamma_{m}
$$

O primeiro limite do segundo membro de (3.119) se anula e " $\left(\Gamma_{m}-\bar{\Gamma}_{\varepsilon}\right)$ " tende a " $\Gamma_{m}$ " quando " $\varepsilon$ " tende a zero. Analisando-se a Fig. 3.11 pode-se escrever (3.119) como:

$$
\int_{\Gamma_{m}} m_{\alpha}^{L} u_{i \alpha}^{*} d \Gamma_{m}=\int_{-l / 2}^{0} m_{\alpha}^{L} u_{i \alpha}^{*} d \Gamma_{m}+\int_{0}^{l / 2} m_{\alpha}^{L} u_{i \alpha}^{*} d \Gamma_{m_{2}}
$$


Mudando-se a variável de " $\Gamma_{m}$ " para " $r$ ", conforme (3.98), a expressão (3.120) fica:

$$
\int_{\Gamma_{m}} m_{\alpha}^{L} u_{i \alpha}^{*} d \Gamma_{m}=\int_{0}^{1 / 2} m_{a}^{L} u_{i \alpha}^{*} d r_{1}+\int_{0}^{1 / 2} m_{a}^{L} u_{i \alpha}^{*} d r_{2}
$$

Analisando-se a equação de " $u_{\alpha \beta}^{*}$ "em (2.32), nota-se que a mesma é função de " $A$ " e " $B$ ", que por sua vez, são funções de " $k_{0}(z)$ " e " $k_{1}(z)$ ", expressas no apêndice $A$. Os valores de " $k_{0}(z)$ " e " $k_{1}(z)$ " são obtidos de formas distintas dependendo-se do valor do argumento " $z=\lambda r^{\text {". Para " }} z \leq 2^{\text {" }}$ as expressōes polinomiais de " $A$ " e " $B$ " são perfeitamente integráveis na variável " $r$ " ; já para " $z$ > 2" será usado um esquema de integração numérica. Assim, (3.121) deve ser escrita como:

$$
\int_{\Gamma_{m}} m_{\beta}^{L} u_{\alpha \beta}^{*} d \Gamma_{m}=\int_{0}^{2 / \lambda} m_{\beta}^{L} u_{\alpha \beta}^{*} d r_{1}+\int_{2 / \lambda}^{l / 2} m_{\beta}^{L} u_{\alpha \beta}^{*} d r_{1}+\int_{0}^{2 / \lambda} m_{\beta}^{L} u_{\alpha \beta}^{*} d r_{2}+\int_{2 / \lambda}^{l / 2} m_{\beta}^{L} u_{\alpha \beta}^{*} d r_{2}
$$

A primeira e terceira parcelas do segundo membro de (3.122) serão integradas analiticamente, já a segunda e quarta serão integradas numericamente, usando-se a quadratura de Gauss.

Analisando-se as parcelas que terão integração analítica, de uma forma genérica, para qualquer dos dois trechos do elemento, a partir de (2.32) tem-se:

$$
\begin{gathered}
\int_{0}^{2 / \lambda} m_{\beta}^{L} u_{\alpha \beta}^{*} d r=\frac{\delta_{\alpha \beta}}{\pi D(1-v)} \int_{0}^{2 / \lambda} m_{\beta}^{L} B d r-\frac{r_{,} r_{, \beta}}{\pi D(1-v)} \int_{0}^{2 / \lambda} m_{\beta}^{L} A d r- \\
\frac{\delta_{\alpha \beta}}{4 \pi D} \int_{0}^{2 / \lambda} m_{\beta}^{L} \ln z d r+\frac{\delta_{\alpha \beta}-2 r_{, a} r_{, \beta}}{8 \pi D} \int_{0}^{2 / \lambda} m_{\beta}^{L} d r
\end{gathered}
$$


Usando-se em (3.123) o valor de " $m_{\beta}^{L " ~ e x p r e s s o ~ c o m o ~ e m ~(3.92), ~ c o m ~}$ os valores de " $\phi_{1}$ ", " $\phi_{2}{ }^{n} e$ " $\phi_{3}$ ", dados em (3.103) ou (3.104), aparecerăo integrais do tipo:

$$
\begin{aligned}
& \int_{0}^{2 / \lambda} A d r \quad, \quad \int_{0}^{2 / \lambda} A r d r \quad, \quad \int_{0}^{2 / \lambda} A r^{2} d r \\
& \int_{0}^{2 / \lambda} B d r \quad, \quad \int_{0}^{2 / \lambda} B r d r \quad, \quad \int_{0}^{2 / \lambda} B r^{2} d r \\
& \int_{0}^{2 / 2} \ln z d r \quad, \quad \int_{0}^{2 / \lambda} r \ln z d r \quad, \quad \int_{0}^{2 / \lambda} r^{2} \ln z d r
\end{aligned}
$$

As últimas integrais de (3.124) são resolvidas pela expressão (3.108). As outras serão calculadas a partir das expansões polinomiais de " $A$ " $e$ " $B$ " (apêndice $A$ ) resultando:

$$
\begin{gathered}
\int_{0}^{2 / \lambda} A r^{n} d r=\left(\frac{2}{\lambda}\right)^{n+1}\left[-\sum_{i=2}^{12,2} \frac{a_{i} t^{* \prime}}{(i+n+1)^{2}}+\sum_{i=2}^{12,2} \frac{b_{i}}{(i+n+1)}-\frac{0,5}{n+1}\right] \\
\int_{0}^{2 / \lambda} B r^{n} d r=\left(\frac{2}{\lambda}\right)^{n+1}\left[-\sum_{i=2}^{12,2} \frac{c_{i} t^{* \prime}}{(i+n+1)^{2}}+\sum_{i=2}^{12,2} \frac{d_{i}}{(i+n+1)}-\frac{0,5386078}{n+1}+\frac{0,5}{(n+1)^{2}}\right]
\end{gathered}
$$

onde:

$$
\begin{aligned}
& A=\ln \left(\frac{z}{2}\right) \sum_{i=2}^{12,2} a_{i} t^{i}+\sum_{i=2}^{12,2} b_{i}\left(\frac{z}{2}\right)^{i}-0,5 \\
& B=-0,5386078-0,5 \ln \left(\frac{z}{2}\right)+\ln \left(\frac{z}{2}\right) \sum_{i=2}^{12,2} c_{i} t^{i}+\sum_{i=2}^{12,2} d_{i}\left(\frac{z}{2}\right)^{i} \\
& t=\frac{z}{3,75}, t^{*}=\frac{2}{3,75}
\end{aligned}
$$




$$
\begin{aligned}
& a_{2}=2 \times 0,87890594-3,5156229 \\
& a_{4}=2 \times 0,51498869-3,0899424 \\
& a_{6}=2 \times 0,15084934-1,2067492 \\
& a_{8}=2 \times 0,02658733-0,2659732 \\
& a_{10}=2 \times 0,00301532-0,0360768 \\
& a_{12}=2 \times 0,00032411-0,0045813 \\
& b_{2}=0,42278420-0,5 \times 0,67278579 \\
& b_{4}=0,23069756-0,5 \times 0,18156897 \\
& b_{6}=0,03488590-0,5 \times 0,01919402 \\
& b_{8}=0,00262698-0,5 \times 0,00110404 \\
& b_{10}=0,00010750-0,5 \times 0,00004686 \\
& b_{12}=0,00000740 \\
& c_{2}=0,87890594-3,5156229 \\
& c_{4}=0,51498869-3,0899424 \\
& c_{6}=0,15084934-1,2067492 \\
& c_{8}=0,02658733-0,2659732 \\
& c_{10}=0,00301532-0,0360768 \\
& c_{12}=0,00032411-0,0045813 \\
& d_{2}=0,42278420-0,25 \times 0,67278579 \\
& d_{4}=0,23069756-0,25 \times 0,18156897 \\
& d_{6}=0,03488590-0,25 \times 0,01919402 \\
& d_{8}=0,00262698-0,25 \times 0,00110404 \\
& d_{10}=0,00010750-0,25 \times 0,00004686 \\
& d_{12}=0,00000740
\end{aligned}
$$

Levando-se (3.124) em (3.123) e somando-se os dois trechos de integração analítica obtém-se: 


$$
\begin{aligned}
& \int_{0}^{2 / \lambda} m_{\beta}^{L} u_{\alpha \beta}^{*} d r=m_{\beta}^{k_{1}}\left\{\frac{4}{\pi D(1-v) l^{2}}\left(\delta_{\alpha \beta} \int_{0}^{2 / \lambda} B r^{2} d r-r_{, \alpha} r_{. \beta} \int_{0}^{2 / \lambda} A r^{2} d r\right)+\right. \\
& \left.\frac{1}{4 \pi D l^{2}}\left[\delta_{a \beta}\left(-4 \int_{0}^{2 / \lambda} r^{2} \ln z d r+\frac{16}{3 \lambda^{3}}\right)-\frac{32 r_{, \alpha} r_{. \beta}}{3 \lambda^{3}}\right]\right\}+ \\
& m_{\beta}^{k_{2}}\left\{\frac{4}{\pi D(1-v)}\left[\delta_{\alpha \beta}\left(2 \int_{0}^{2 / \lambda} B d r-\frac{8}{l^{2}} \int_{0}^{2 / \lambda} B r^{2} d r\right)-r_{, \alpha} r_{, \beta}\left(2 \int_{0}^{2 / \lambda} A d r-\frac{8}{l^{2}} \int_{0}^{2 / \lambda} A r^{2} d r\right)\right]+\right. \\
& \left.\frac{1}{4 \pi D}\left[\delta_{\alpha \beta}\left(-2 \int_{0}^{2 / \lambda} \ln z d r+\frac{8}{l^{2}} \int_{0}^{2 / \lambda} r^{2} \ln z d r+\frac{2}{\lambda}-\frac{32}{3 \lambda^{3} l^{2}}\right)-r_{, \alpha} r_{, \beta}\left(\frac{4}{\lambda}-\frac{64}{3 \lambda^{3} l^{2}}\right)\right]\right\}+ \\
& m_{\beta}^{k_{3}}\left\{\frac{4}{\pi D(1-v) l^{2}}\left(\delta_{\alpha \beta} \int_{0}^{2 / \lambda} B r^{2} d r-r_{, \alpha} r_{, \beta} \int_{0}^{2 / \lambda} A r^{2} d r\right)+\right. \\
& \left.\frac{1}{4 \pi D l^{2}}\left[\delta_{\alpha \beta}\left(-4 \int_{0}^{2 / \lambda} r^{2} \ln z d r+\frac{16}{3 \lambda^{3}}\right)-\frac{32}{3 \lambda^{3}} r_{, \alpha} r_{, \beta}\right]\right\}
\end{aligned}
$$

onde " $m_{\beta}^{k}$ " são os valores nodais da linha de momentos " $\mathrm{m}_{\beta}$ ".

Analisando-se a integração numérica para o trecho 1 do elemento de carga "k" (Fig. 3.11) vem:

$$
\begin{aligned}
& \int_{2 / \lambda}^{1 / 2} m_{\beta}^{L} u_{\alpha \beta}^{*} d r_{1}=-\int_{-2 / \lambda}^{-1 / 2} m_{\beta}^{L} u_{\alpha \beta}^{*} d \Gamma=-\frac{l}{2} \int_{-4 / \lambda l}^{-1} m_{\beta}^{L} u_{\alpha \beta}^{*} d \eta= \\
& -\frac{\lambda l-4}{4 \lambda} \int_{+1}^{-1} m_{\beta}^{L} u_{\alpha \beta}^{*} d \eta_{1}=\frac{\lambda l-4}{4 \lambda} \int_{-1}^{+1} m_{\beta}^{L} u_{\alpha \beta}^{*} d \eta_{1}
\end{aligned}
$$

onde " $\eta_{1}$ " é uma nova coordenada homogênea do trecho 1 , valendo para valores de " $r$ ", entre " $2 / \lambda$ " e "l/2". Esta nova coordenada pode ser escrita em função de " $\eta$ ", como se segue:

$$
\begin{aligned}
& \eta_{1}=\frac{1}{\lambda l-4}(2 \lambda l \eta+\lambda l+4) \\
& d \eta_{1}=\frac{2 \lambda l}{\lambda l-4} d \eta
\end{aligned}
$$




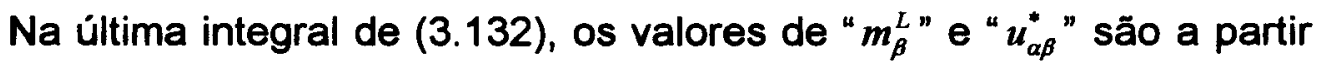
de (3.133), funções da nova coordenada " $\eta_{1}$ ", podendo-se integrá-la numericamente.

Analogamente para o trecho 2 do elemento de carga pode-se escrever:

$$
\begin{aligned}
& \eta_{2}=\frac{1}{\lambda l-4}(2 \lambda l \eta-\lambda l-4) \\
& d \eta_{2}=\frac{2 \lambda l}{\lambda l-4} d \eta
\end{aligned}
$$

ficando a integração numérica neste caso dada por:

$$
\int_{2 / \lambda}^{l / 2} m_{\beta}^{L} u_{\alpha \beta}^{*} d r_{2}=\frac{\lambda l-4}{4 \lambda} \int_{-1}^{+1} m_{\beta}^{L} u_{\alpha \beta}^{*} d \eta_{2}
$$

Para completar a contribuição da linha de momentos " $m_{\alpha}$ ", no cálculo dos deslocamentos dos pontos internos, quando " $\xi=k_{2}$ " falta analisar 0 termo:

$$
\int_{\Gamma_{m}} m_{\alpha}^{L} u_{3 \alpha}^{*} d \Gamma_{m}=\int_{0}^{l / 2} m_{\alpha}^{L} u_{3 \alpha}^{*} d r_{1}+\int_{0}^{l / 2} m_{\alpha}^{L} u_{3 \alpha}^{*} d r_{2}
$$

que depois de efetuado a soma resulta:

$$
\begin{aligned}
& \int_{\Gamma_{m}} m_{\alpha}^{L} u_{3 \alpha}^{*} d \Gamma_{m}=\frac{r_{, \alpha}}{4 \pi D}\left\{\left[\frac{2}{l} \int_{0}^{l / 2} r^{2} \ln z d r-\frac{l^{2}}{12}\right] m_{\alpha}^{k_{1}}+\right. \\
& \left.\left[-\frac{2}{l} \int_{0}^{l / 2} r^{2} \ln z d r+\frac{l^{2}}{12}\right] m_{\alpha}^{k_{3}}\right\}
\end{aligned}
$$

onde " $r_{, \alpha}$ " é dado na expressão (3.105). 
106

Será analisada agora a contribuição da linha de momentos " $m_{\alpha}$ ", no cálculo dos deslocamentos em pontos internos, quando o ponto fonte " $\xi$ " coincide com o ponto nodal " $k_{1}$ " (ver Fig. 3.12).

De forma análoga ao apresentado para " $\xi=k_{2}$ ", pode-se escrever quando " $\xi=k_{1}$ ":

$$
\int_{\Gamma_{m}} m_{\beta}^{L} u_{\alpha \beta}^{*} d \Gamma_{m}=\int_{0}^{2 / \lambda} m_{\beta}^{L} u_{\alpha \beta}^{*} d r+\int_{2 / \lambda}^{l} m_{\beta}^{L} u_{\alpha \beta}^{*} d r
$$

onde $o$ primeiro termo é integrado analiticamente e 0 segundo numericamente, sendo expressos respectivamente por:

$$
\begin{aligned}
& \int_{0}^{2 / \lambda} m_{\beta}^{L} u_{\alpha \beta}^{*} d r=m_{\beta}^{k_{1}}\left\{\frac { 1 } { \pi D ( 1 - v ) } \left[\delta_{\alpha \beta}\left(\frac{2}{l^{2}} \int_{0}^{2 / \lambda} B r^{2} d r-\frac{3}{l} \int_{0}^{2 / \lambda} B r d r+\int_{0}^{2 / \lambda} B d r\right)-\right.\right. \\
& r_{, \alpha} r_{, \beta}\left(\frac{2}{l^{2}} \int_{0}^{2 / \lambda} A r^{2} d r-\frac{3}{l} \int_{0}^{2 / \lambda} A r d r+\int_{0}^{2 / \lambda} A d r\right)+ \\
& \frac{1}{4 \pi D}\left[\delta_{\alpha \beta}\left(-\frac{2}{l^{2}} \int_{0}^{2 / \lambda} r^{2} \ln z d r+\frac{3}{l} \int_{0}^{2 / \lambda} r \ln z d r+\frac{8}{3 \lambda^{3} l^{2}}+\frac{3}{\lambda^{2} l}+\frac{1}{\lambda}\right)-\right. \\
& \left.\left.r_{, \alpha} r_{, \beta}\left(\frac{16}{3 \lambda^{3} l^{2}}-\frac{6}{\lambda^{2} l}+\frac{2}{\lambda}\right)\right]\right\}+ \\
& m_{\beta}^{k_{2}}\left\{\frac { 1 } { \pi D ( 1 - v ) } \left[\delta_{\alpha \beta}\left(\frac{4}{l} \int_{0}^{2 / \lambda} B d r-\frac{4}{l^{2}} \int_{0}^{2 / \lambda} B r^{2} d r\right)-\right.\right. \\
& \left.\left.r_{, \alpha} r_{, \beta}\left(\frac{4}{l} \int_{0}^{2 / \lambda} A r d r-\frac{4}{l^{2}} \int_{0}^{2 / \lambda} A r^{2} d r\right)\right]+139\right) \\
& \left.\frac{1}{4 \pi D}\left[\delta_{\alpha \beta}\left(-\frac{4}{l} \int_{0}^{2 / \lambda} r \ln z d r+\frac{4}{l^{2}} \int_{0}^{2 / \lambda} r^{2} \ln z d r+\frac{8}{\lambda^{2} l}-\frac{16}{3 \lambda^{3} l^{2}}\right)-r_{, \alpha} r_{, \beta}\left(\frac{16}{\lambda^{2} l}-\frac{32}{3 \lambda^{3} l^{2}}\right)\right]\right\}+ \\
& m_{\beta}^{k_{3}}\left\{\frac{1}{\pi D(1-v)}\left[\delta_{\alpha \beta}\left(\frac{2}{l^{2}} \int_{0}^{2 / \lambda} B r^{2} d r-\frac{1}{l} \int_{0}^{2 / \lambda} B r d r\right)-r_{, \alpha^{\prime}, \beta}\left(\frac{2}{l^{2}} \int_{0}^{2 / \lambda} A r^{2} d r-\frac{1}{l} \int_{0}^{2 / \lambda} A r d r\right)\right]+\right. \\
& \left.\frac{1}{4 \pi D}\left[\delta_{\alpha \beta}\left(-\frac{2}{l^{2}} \int_{0}^{2 / \lambda} r^{2} \ln z d r+\frac{1}{l} \int_{0}^{2 / \lambda} r \ln z d r+\frac{8}{3 \lambda^{3} l}-\frac{1}{\lambda^{2} l}\right)-r_{, \alpha} r_{, \beta}\left(\frac{16}{3 \lambda^{3} l^{2}}-\frac{2}{\lambda^{2} l}\right)\right]\right\}
\end{aligned}
$$


107

$$
\int_{2 / \lambda}^{l} m_{\beta}^{L} u_{\alpha \beta}^{*} d r=\frac{\lambda l-2}{2 \lambda} \int_{-1}^{+1} m_{\beta}^{L} u_{\alpha \beta}^{*} d \eta_{1}
$$

com:

$$
\eta_{1}=\frac{1}{\lambda l-2}(\lambda l \eta-2)
$$

$$
d \eta_{1}=\frac{\lambda l}{\lambda l-2} d \eta
$$

Escrevendo-se (3.136) para o caso em questão " $\xi=k_{1}$ ", tem-se:

$$
\int_{\Gamma_{m}} m_{\alpha}^{L} u_{3 \alpha}^{*} d \Gamma_{m}=\int_{0}^{l} m_{\alpha}^{L} u_{3 \alpha}^{*} d r
$$

Integrando-se analiticamente (3.142) obtém-se:

$$
\begin{aligned}
& \int_{\Gamma_{m}} m_{\alpha}^{L} u_{3 \alpha}^{*} d \Gamma_{m}=\frac{r_{a}}{4 \pi D}\left\{\left(-\frac{2}{l^{2}} \int_{0}^{l} r^{3} \ln z d r+\frac{3}{l} \int_{0}^{l} r^{2} \ln z d r-\int_{0}^{l} r \ln z d r\right) m_{\alpha}^{k_{1}}+\right. \\
& \left(-\frac{4}{l} \int_{0}^{l} r^{2} \ln z d r+\frac{4}{l^{2}} \int_{0}^{l} r^{3} \ln z d r+\frac{l^{2}}{3}\right) m_{\alpha}^{k_{2}}+ \\
& \left.\left(-\frac{2}{l^{2}} \int_{0}^{l} r^{3} \ln z d r+\frac{1}{l} \int_{0}^{l} r^{2} \ln z d r+\frac{l^{2}}{6}\right) m_{\alpha}^{k_{3}}\right\}
\end{aligned}
$$

onde " $r_{,} \alpha$ " é o valor expresso em (3.105).

Finalmente será analisada a contribuição das linhas de momentos " $\mathrm{m}_{\alpha}{ }$, no cálculo dos deslocamentos dos pontos internos, quando o ponto fonte " $\xi$ ", coincide com o ponto nodal " $k_{3}$ " (ver Fig. 3.13).

Analogamente ao desenvolvido anteriormente para " $\xi=k_{1}$ ", pode-se escrever para " $\xi=k_{3}{ }^{n}$ :

$$
\int_{0}^{2 / \lambda} m_{\beta}^{L} u_{\alpha \beta}^{*} d \Gamma_{m}=m_{\beta}^{k_{1}}\left\{\frac { 1 } { \pi D ( 1 - v ) } \left[\delta_{a \beta}\left(\frac{2}{l^{2}} \int_{0}^{2 / \lambda} B r^{2} d r-\frac{1}{l} \int_{0}^{2 / \lambda} B r d r\right)-\right.\right.
$$


108

$$
\begin{aligned}
& \left.r_{, \alpha} r_{, \beta}\left(\frac{2}{l^{2}} \int_{0}^{2 / \lambda} A r^{2} d r-\frac{1}{l} \int_{0}^{2 / \lambda} A r d r\right)\right]+ \\
& \frac{1}{4 \pi D}\left[\delta_{\alpha \beta}\left(-\frac{2}{l^{2}} \int_{0}^{2 / \lambda} r^{2} \ln z d r+\frac{1}{l} \int_{0}^{2 / \lambda} r \ln z d r+\frac{8}{3 \lambda^{3} l^{2}}-\frac{1}{\lambda^{2} l}\right)-\right. \\
& \left.\left.r_{, \alpha} r_{, \beta}\left(\frac{16}{3 \lambda^{3} l^{2}}-\frac{2}{\lambda^{2} l}\right)\right]\right\}+ \\
& m_{\beta}^{k_{2}}\left\{\frac { 1 } { \pi D ( 1 - v ) } \left[\delta_{\alpha \beta}\left(\frac{4}{l} \int_{0}^{2 / \lambda} B r d r-\frac{4}{l^{2}} \int_{0}^{2 / \lambda} B r^{2} d r\right)-\right.\right. \\
& \left.r_{, \alpha} r_{, \beta}\left(\frac{4}{l} \int_{0}^{2 / \lambda} A r d r-\frac{4}{l^{2}} \int_{0}^{2 / \lambda} A r^{2} d r\right)\right]+ \\
& \frac{1}{4 \pi D}\left[\delta_{\alpha \beta}\left(-\frac{4}{l} \int_{0}^{2 / \lambda} r \ln z d r+\frac{4}{l^{2}} \int_{0}^{2 / \lambda} r^{2} \ln z d r+\frac{4}{\lambda^{2} l}-\frac{16}{3 \lambda^{3} l^{2}}\right)-\right. \\
& \left.\left.r_{, \alpha} r_{, \beta}\left(\frac{8}{\lambda^{2} l}-\frac{32}{3 \lambda^{3} l^{2}}\right)\right]\right\}+ \\
& m_{\beta}^{k_{3}}\left\{\frac { 1 } { \pi D ( 1 - v ) } \left[\delta_{\alpha \beta}\left(\frac{2}{l^{2}} \int_{0}^{2 / \lambda} B r^{2} d r-\frac{3}{l} \int_{0}^{2 / \lambda} B r d r+\int_{0}^{2 / \lambda} B d r\right)-\right.\right. \\
& \left.r_{. \alpha} r_{. \beta}\left(\frac{2}{l^{2}} \int_{0}^{2 / \lambda} A r^{2} d r-\frac{3}{l} \int_{0}^{2 / \lambda} A r d r+\int_{0}^{2 / \lambda} A d r\right)\right]+ \\
& \frac{1}{4 \pi D}\left[\delta_{\alpha \beta}\left(-\frac{2}{l^{2}} \int_{0}^{2 / \lambda} r^{2} \ln z d r+\frac{3}{l} \int_{0}^{2 / \lambda} r \ln z d r-\int_{0}^{2 / \lambda} \ln z d r+\frac{8}{3 \lambda^{3} l^{2}}-\frac{3}{\lambda^{2} l}+\frac{1}{\lambda}\right)-\right. \\
& \left.\left.r_{, \alpha} r_{, \beta}\left(\frac{16}{3 \lambda^{3} l^{2}}-\frac{6}{\lambda^{2} l}+\frac{2}{\lambda}\right)\right]\right\} \\
& \int_{2 / \lambda}^{l} m_{\beta}^{L} u_{\alpha \beta}^{*} d r=\frac{\lambda l-2}{2 \lambda} \int_{-1}^{+1} m_{\beta}^{L} u_{\alpha \beta}^{*} d \eta_{1}
\end{aligned}
$$

com:

$$
\eta_{1}=\frac{1}{\lambda l-2}(\lambda l \eta+2)
$$

$$
d \eta_{1}=-\frac{\lambda l}{\lambda l-2} d \eta
$$


Escrevendo-se (3.143) para " $\xi=k_{3}$ " obtém-se:

$$
\begin{aligned}
& \int_{\Gamma_{a}} m_{a}^{L} u_{3 \alpha}^{*} d \Gamma_{m}=\frac{r_{a}}{4 \pi D}\left\{\left(-\frac{2}{l^{2}} \int_{0}^{l} r^{3} \ln z d r+\frac{1}{l} \int_{0}^{l} r^{2} \ln z d r-\frac{l^{2}}{12}\right) m_{a}^{k_{1}}+\right. \\
& \left(-\frac{4}{l} \int_{0}^{l} r^{2} \ln z d r+\frac{4}{l^{2}} \int_{0}^{l} r^{3} \ln z d r-\frac{l^{2}}{6}\right) m_{a}^{k_{2}}+ \\
& \left.\left(-\frac{2}{l^{2}} \int_{0}^{l} r^{3} \ln z d r+\frac{3}{l} \int_{0}^{l} r^{2} \ln z d r+\int_{0}^{l} \ln z d r\right) m_{a}^{k_{3}}\right\}
\end{aligned}
$$

onde " $r, \alpha$ " é dado na expressão ( 3.118 ).

\subsection{5 - ESFORÇOS NOS PONTOS INTERNOS}

O cálculo dos momentos e forças cortantes para pontos do domínio é feito através das equaçōes (3.26) e (3.27) respectivamente. Nestas equaçōes, vê-se que os esforços estão expressos em função dos valores dos deslocamentos e forças de superfície para pontos " $X$ " do contorno " $\Gamma$ " da placa, além de receberem a influência dos carregamentos de dominio. Dividindo-se o contorno em elementos, conforme mostrado no item 3.3.2, expressando-se os valores de contorno conforme (3.76), consegue-se escrever de forma discretizada as equações (3.26) e (3.27), que serão dadas por:

$$
\begin{aligned}
& \mathbf{M}_{\alpha \beta}(\xi)=\sum_{j=1}^{N E C P} \frac{1}{2} \int_{-1}^{+1} u_{\alpha \beta k}^{*}(\xi, X) \Psi^{T}(\eta) d \eta \underset{\sim k(j)}{P_{N}^{N}}-\sum_{j=1}^{N E C P} \frac{j}{2} \int_{-1}^{+1} p_{\alpha \beta k}^{*}(\xi, X) \Psi^{T}(\eta) d \eta \underset{\sim k(j)}{U^{N}}+ \\
& \int_{\Omega_{q}} w_{\alpha \beta}^{*}(\xi, x) q(x) d \Omega_{q}(x)+\int_{\Omega_{m}} u_{\alpha \beta \gamma}^{*}(\xi, x) m_{\gamma}(x) d \Omega_{m}(x)+\frac{v}{(1-v) \lambda^{2}} q(\xi) \delta_{\alpha \beta}
\end{aligned}
$$




$$
\begin{aligned}
& Q_{3 \beta}(\xi)=\sum_{j=1}^{N E C P} \frac{1_{j}}{2} \int_{-1}^{+1} u_{3 \beta k}^{*}(\xi, X) \Psi^{T}(\eta) d \eta \underset{\sim k(j)}{P^{N}}-\sum_{j=1}^{N E C P} \frac{j}{2} \int_{-1}^{+1} p_{3 \beta k}^{*}(\xi, X) \Psi^{T}(\eta) d \eta \underset{\sim k(j)}{U^{N}}+ \\
& \int_{\Omega_{q}} w_{3 \beta}^{*}(\xi, x) q(x) d \Omega_{q}(x)+\int_{\Omega_{m}} u_{3 \beta \gamma}^{*}(\xi, x) m_{\gamma}(x) d \Omega_{m}(x)
\end{aligned}
$$

As duas primeiras integrais dos segundos membros de (3.148) e (3.149) são análogas às integrais (3.83), ou seja, elas expressam a influência dos valores nodais de contorno sobre o cálculo dos esforços internos no ponto " $\xi$ ". Elas serão calculadas numericamente, através de quadratura de Gauss, obtendo-se coeficientes de influência, análogos aos " $g_{i j}$ " e " $h_{i j}$, expressos por:

$$
\begin{aligned}
& g_{a \beta k}^{\prime n}(\xi)=\frac{l_{j}}{2} \int_{-1}^{+1} u_{\alpha \beta k}^{*}(\xi, X) \psi_{n}(\eta) d \eta(X) \\
& h_{a \beta k}^{\prime n}(\xi)=\frac{l_{j}}{2} \int_{-1}^{+1} p_{\alpha \beta k}^{*}(\xi, X) \psi_{n}(\eta) d \eta(X) \\
& g_{3 \beta k}^{\prime n}(\xi)=\frac{l_{j}}{2} \int_{-1}^{+1} u_{3 \beta k}^{*}(\xi, X) \psi_{n}(\eta) d \eta(X) \\
& h_{3 \beta k}^{\prime n}(\xi)=\frac{l_{j}}{2} \int_{-1}^{+1} p_{3 \beta k}^{*}(\xi, X) \psi_{n}(\eta) d \eta(X)
\end{aligned}
$$

onde os coeficientes (3.150) se referem aos momentos e os (3.51) às forças cortantes.

Escrevendo-se as equaçōes (3.148) e (3.149) para os "NPI" pontos internos que se tenha interesse no cálculo dos esforços, os coeficientes

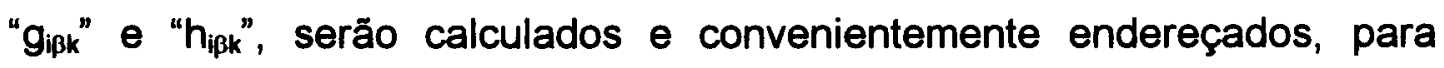
formar respectivamente as matrizes G' e H'. Estas matrizes serão de ordem [5(NPI)X3(NNCP)], sendo gerado para cada ponto interno " $\xi$ " o seguinte vetor de esforços: 


$$
\stackrel{\sim}{\xi}_{\sim}^{\xi}=\left\{\begin{array}{l}
M_{11} \\
M_{12} \\
M_{22} \\
Q_{31} \\
Q_{32}
\end{array}\right\}
$$

As equações (3.148) e (3.149) escritas para todos os "NPI" pontos internos, podem ser representadas matricialmente por:

$$
\underset{\sim}{E}=\underset{\sim}{G^{\prime}} \underset{\sim}{P} \underset{\sim}{H} \underset{\sim}{U}+\underline{\sim}^{\prime}
$$

onde:

$\underset{\sim}{E}$ - é o vetor dos esforços internos 5(NPI)x1.

$\underset{\sim}{G} e \underset{\sim}{H}$ 'são as matrizes contendo os coeficientes " $g_{\mathrm{ipk}}^{\prime}$ " e " $\mathrm{h}$ 'ipk" $5(\mathrm{NPI}) \times 3(\mathrm{NNCP})$.

Ue $P$ - são os valores nodais dos deslocamentos e forças de superfície, respectivamente $-3(\mathrm{NNCP}) \times 1$.

$\underline{\sim}^{\prime \prime}$ - é o vetor que contém a influência dos carregamentos internos no cálculo dos esforços - 5(NPI)x1.

A influência da carga distribuída " $q(x)$ " atuando em " $\Omega_{q}$ ", no cálculo dos esforços em pontos internos é dada pelas expressões (3.35), quando " $q(x)$ " é constante, ou pelas expressões (3.46), quando " $q(x)$ " assume distribuição linear. Em ambos os casos a integral sobre o domínio " $\Omega_{q}$ " é transformada em uma integral sobre o contorno " $\Gamma_{\mathrm{q}}$. Considerando-se este contorno discretizado em elementos retos conforme item 3.3.3, pode-se escrever as equações (3.35) e (3.46) na forma discretizada como: 


$$
\begin{aligned}
& b_{i}^{\prime}(\xi)=q(x) \sum_{j=1}^{N E C C} \frac{l_{j}}{2} \int_{-1}^{+1} W_{\alpha \beta}^{*}(\xi, x) d \eta \\
& b_{\beta+3}^{\prime}(\xi)=q(x) \sum_{j=1}^{N E C C} \frac{l_{j}}{2} \int_{-1}^{+1} W_{3 \beta}^{*}(\xi, x) d \eta
\end{aligned}
$$

para o caso de " $q(x)^{n}$ constante, ou:

$$
\begin{aligned}
& b_{i}^{\prime}(\xi)=\sum_{j=1}^{N E C C} \frac{l_{j}}{2} \int_{-1}^{+1} q(x) I_{\alpha \beta}^{*}(\xi, x) d \eta \\
& b_{\beta+3}^{\prime}(\xi)=\sum_{j=1}^{N E C C} \frac{l_{j}}{2} \int_{-1}^{+1} q(x) I_{3 \beta}^{*}(\xi, x) d \eta
\end{aligned}
$$

quando " $q(x)$ " for linear.

As soluçōes fundamentais " $W_{i \beta}^{*}$ e " $I_{i \beta}^{*}$ " estão apresentadas respectivamente nas expressões (3.37) e (3.47), e "NECC" é o número de elementos do contorno da carga.

$O$ vetor " $B^{\prime \prime}$ em (3.153) é formado pela contribuição dos coeficientes " $b_{i}^{\prime \prime} e{ }^{\prime \prime} b_{\beta+3}^{\prime}$ " apresentadas em (3.154) ou (3.155). Os primeiros se referem aos três momentos " $M_{11}$ ", " $M_{12}$ " e " $M_{22}$ " e estarão armazenadas em " $B_{\sim}$ ", nas três primeiras linhas do ponto interno " $\xi$ ". Os coeficientes " $b_{\beta+3}^{\prime}$ ", referentes às forças cortantes " $Q_{31}$ " e " $Q_{32}$ ", estarão armazenados em " $B^{\prime}$ ", na quarta e quinta linhas respectivamente, do mesmo ponto interno. 
A influência da aplicação de uma carga concentrada " $\mathrm{P}$ " no ponto " $x$ ", no cálculo dos esforços no ponto interno " $\xi$ ", é obtida diretamente pelas seguintes expressões:

$$
\begin{aligned}
& b_{i}^{\prime}(\xi)=P w_{\alpha \beta}^{*}(\xi, x) \\
& b_{\beta+3}^{\prime}(\xi)=P w_{3 \beta}^{*}(\xi, x)
\end{aligned}
$$

onde " $w_{i \beta}^{*}(\xi, x)$ " é dado nas expressōes (3.31).

Para o caso em que " $\xi=x "$, ou seja, o ponto onde se deseja os esforços coincide com o ponto de aplicação da carga " $P$ ", os valores de (3.156) serão considerados na situaçăo limite quando " $r$ " tende a zero. Analisando-se as expressões (3.31) tem-se:

$$
\begin{aligned}
& b_{i}^{\prime}(\xi)=P \lim _{r \rightarrow 0} w_{\alpha \beta}^{*}(\xi, x)=-\frac{v \delta_{\alpha \beta}}{\lambda^{2}} P \\
& b_{\beta+3}^{\prime}(\xi)=P \lim _{r \rightarrow 0} w_{3 \beta}^{*}(\xi, x)=0
\end{aligned}
$$

Os valores de " $b_{i}^{\prime n} e$ " $b_{\beta+3}^{\prime}$ " convenientemente armazenados e endereçados, levam a contribuição da carga concentrada " $P$ ", na formação do vetor " $B$ ".

A contribuição da linha de carga distribuída " $q_{L}(x)$ ", no cálculo dos esforços internos, expressa em (3.51), será escrita de uma maneira discretizada como: 


$$
\begin{aligned}
& b_{i}{ }^{\prime}(\xi)=\sum_{j=1}^{N E L C} \frac{l_{j}}{2} \int_{-1}^{+1} w_{\alpha \beta}^{*}(\xi, x) \underset{\sim q}{\psi_{q}^{T}}(\eta) d \eta \underset{\sim L}{q^{N}}
\end{aligned}
$$

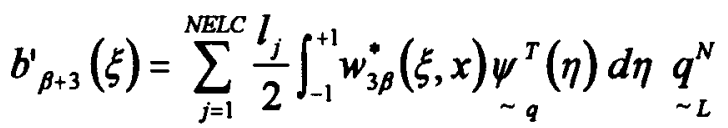

onde " $\underset{\sim}{\psi_{q}}(\eta)$ " é dado em (3.90) e " $\underset{\sim}{q_{L}^{N}}$ são os valores nodais da carga " $q_{L}$ ".

Os coeficientes (3.158) dão a contribuição da carga distribuída em linha "qL", no cálculo dos momentos $\left(b_{i}^{\prime}\right)$ e cortantes $\left(b_{\beta+3}^{\prime}\right)$ em pontos internos " $\xi$ ", e serão endereçados e armazenados convenientemente no vetor " $B$ " em (3.153).

Caso o ponto " $\xi$ " coincida com um dos pontos " $k_{1}$ ", " $k_{2}$ " ou " $k_{3}$ " do elemento "j", as integrais (3.158) deverão ser calculadas analiticamente sobre o elemento "j". Será analisada inicialmente a contribuição da linha de carga na determinação dos momentos, ou seja, a primeira das expressões (3.158). Conforme visto no item 3.2.5, quando o ponto " $\xi$ " coincide com a linha de carga, a contribuição desta no cálculo dos momentos é dada pela equação (3.56). A equação (3.56) escrita para a situação em que " $\xi=k_{2}$ ", conforme Fig.3.11, resulta:

$$
\int_{\Gamma_{q}} q_{L} w_{\alpha \beta}^{*} d \Gamma_{q}=\int_{-l / 2}^{0} q_{L} w_{\alpha \beta}^{*} d \Gamma_{1}+\int_{0}^{l / 2} q_{L} w_{\alpha \beta}^{*} d \Gamma_{2}
$$

onde as letras $(x)$ e $(\xi, x)$ foram suprimidas, apenas por simplificação.

Considerando-se " $\mathrm{q}_{\mathrm{L}}$ " como em (3.90), com as componentes " $\phi_{i}$ " dadas, em (3.103) e (3.104), tem-se:

$$
\int_{\Gamma_{q}} q_{L} w_{a \beta}^{*} d \Gamma_{q}=\left(q_{l}^{k_{1}}+q_{l}^{k_{3}}\right)\left\{\frac{\delta_{\alpha \beta}}{4 \pi}\left[-\frac{4(1+v)}{l^{2}} \int_{0}^{l / 2} r^{2} \ln z d r+\frac{(1-v) l}{12}-\frac{4 v}{\lambda^{2} l}\right]+\right.
$$




$$
\begin{aligned}
& \left.\frac{r_{, \alpha} r_{, \beta}}{4 \pi}\left[\frac{8 v}{\lambda^{2} l}-(1-v) \frac{l}{6}\right]\right\}+q_{l}^{k_{2}}\left\{\frac { \delta _ { \alpha \beta } } { 4 \pi } \left[2(1+v)\left(\frac{4}{l^{2}} \int_{0}^{l / 2} r^{2} \ln z d r-\int_{0}^{l / 2} \ln z d r\right)+\right.\right. \\
& \left.\left.(1-v) \frac{21}{3}+\frac{4 v}{\lambda^{2}}\left(\frac{4}{1}-\lim _{\varepsilon \rightarrow 0} \frac{1}{\varepsilon}\right)\right]+\frac{2 v r_{, \alpha} r_{, \beta}}{\lambda^{2} \pi}\left(-\frac{4}{1}+\lim _{\varepsilon \rightarrow 0} \frac{1}{\varepsilon}\right)\right\}
\end{aligned}
$$

onde " $q_{L}^{k_{1}}$ " é o valor da carga " $q_{L}$ " no ponto nodal " $k_{i}^{n}$; " $r_{, \alpha}$ " é dado na expressão (3.105) e as integrais em "In z" estão expressas em (3.108).

Para se obter os coeficientes " $b_{i}^{\prime}$, deve-se integrar numericamente em todos os elementos de " $\Gamma_{q}$ ", com exceção do elemento " $j$ ", onde a contribuição para o " $b_{i}^{\prime}$ " se faz através da expressão (3.160).

Os coeficientes " $b_{\beta+3}^{\prime}$ " para o caso em que o ponto " $\xi$ " coincida com o ponto " $k_{2}$ " (Fig. 3.11), são obtidos pela soma das integrais numéricas sobre todos os elementos de " $\Gamma_{q}$ ", exceto para o elemento "j", que será integrado analiticamente. Estes coeficientes representam a contribuição da carga " $\mathrm{q}_{\mathrm{L}}$ " no cálculo das forças cortantes em pontos internos. Segundo o item 3.2.5, esta contribuição para o caso do elemento "j" conter o nó singular "६̧", é expressa em (3.62), que será agora reescrita para a situação " $\xi=k_{2}$ ", resultando:

$$
\int_{\Gamma_{q}} q_{L} w_{3 \beta}^{*} d \Gamma_{q}=q_{L}^{k_{2}} \frac{n_{\beta}}{2 \pi}+\int_{-l / 2}^{0} q_{L} w_{3 \beta}^{*} d \Gamma_{1}+\int_{0}^{l / 2} q_{L} w_{3 \beta}^{*} d \Gamma_{2}
$$

Integrando-se analiticamente (3.161) para " $q_{L}$ " dado em (3.90), com as componentes " $\phi_{i}$ " dadas em (3.103) e (3.104), obtém-se:

$$
\int_{\Gamma_{q}} q_{L} u_{3 \beta}^{*} d \Gamma_{q}=\frac{1}{2 \pi}\left[-r_{, \beta} q_{L}^{k_{1}}+n_{\beta} q_{L}^{k_{2}}+r_{, \beta} q_{L}^{k_{3}}\right]
$$


onde " $n_{\beta}$ " é dado em (3.61) e " $r_{, \beta}$ " em (3.105).

Quando o ponto " $\xi$ " coincide com o ponto nodal " $k_{1}$ ", do elemento "j", conforme ilustrado na Fig. 3.12, a contribuiçăo da linha de carga " $q_{L}(x)$ " no cálculo dos momentos internos " $\mathrm{M}_{\alpha \beta}$ ”, é dada por uma expressão análoga à (3.159):

$$
\int_{\Gamma_{q}} q_{L} w_{\alpha \beta}^{*} d \Gamma_{q}=\int_{-l / 2}^{l / 2} q_{L} w_{\alpha \beta}^{*} d \Gamma=\int_{0}^{l} q_{L} w_{\alpha \beta}^{*} d r
$$

Considerando-se " $\mathrm{qL}_{\mathrm{L}}$ " como em (3.90), com as componentes " $\phi_{i}{ }^{\prime}$ dadas em (3.111), tem-se:

$$
\begin{aligned}
& \int_{\Gamma_{q}} q_{L} w_{\alpha \beta}^{*} d \Gamma_{q}=q_{L}^{k_{L}}\left\{\frac{(1+v) \delta_{\alpha \beta}}{4 \pi}\left[-\frac{2}{l^{2}} \int_{0}^{l} r^{2} \ln z d r+\frac{3}{l} \int_{0}^{l} r \ln z d r-\int_{0}^{l} \ln z d r\right]+\right. \\
& \left.\frac{(1-v)\left(\delta_{\alpha \beta}-2 r_{, \alpha} r_{, \beta}\right)}{48 \pi} l+\frac{v\left(2 r_{, \alpha} r_{, \beta}-\delta_{\alpha \beta}\right)}{2 \pi \lambda^{2}}\left(\frac{1}{l}-\frac{3}{l} \ln l+\frac{3}{l} \lim _{\delta \rightarrow 0} \ln \varepsilon+\lim _{\varepsilon \rightarrow 0} \frac{1}{\varepsilon}\right)\right\}+ \\
& q_{L}^{k_{2}}\left\{\frac{(1+v) \delta_{\alpha \beta}}{\pi}\left(\frac{1}{l^{2}} \int_{0}^{l} r^{2} \ln z d r-\frac{1}{l} \int_{0}^{l} r \ln z d r\right)+\frac{(1-v)\left(\delta_{\alpha \beta}-2 r_{, \alpha} r_{, \beta}\right)}{12 \pi} l+\right. \\
& \left.\frac{2 v\left(2 r_{, \alpha} r_{, \beta}-\delta_{\alpha \beta}\right)}{\pi \lambda^{2} l}\left(\ln l-\lim _{\varepsilon \rightarrow 0} \ln \varepsilon-1\right)\right\}+ \\
& q_{L}^{k_{3}}\left\{\frac{(1+v) \delta_{\alpha \beta}}{4 \pi}\left(-\frac{2}{l^{2}} \int_{0}^{l} r^{2} \ln z d r+\frac{1}{l} \int_{0}^{l} r \ln z d r\right)+\right. \\
& \left.\frac{(1-v)\left(\delta_{\alpha \beta}-2 r_{, \alpha} r_{, \beta}\right)}{48 \pi} l+\frac{v\left(2 r_{, \alpha} r_{, \beta}-\delta_{\alpha \beta}\right)}{2 \pi \lambda^{2} l}\left(2-\ln l+\lim _{\varepsilon \rightarrow 0} \ln \varepsilon\right)\right\}
\end{aligned}
$$

onde " $q_{L}^{k_{1}}$ " é o valor da carga " $q_{L}$ " no ponto nodal " $k_{i}$ "; "r,a" é dado na expressão (3.105) e as integrais em "In z" estão expressas em (3.108). 
117

Considerando-se a contribuição de " $\mathrm{qL}_{\mathrm{L}}$ " no cálculo das forças cortantes quando o ponto " $\xi$ " coincide com o nó " $k_{1}$ " do elemento "j", pode-se escrever analogamente a expressăo (3.161):

$$
\int_{\Gamma_{q}} q_{L} w_{3 \beta}^{*} d \Gamma_{q}=q_{L}^{k_{1}} \frac{n_{\beta}}{2 \pi}+\int_{0}^{l} q_{L} w_{3 \beta}^{*} d \Gamma
$$

Integrando-se analiticamente (3.165) para " $q_{L}$ " dado em (3.90), com as componentes " $\phi_{i}$ " dadas em (3.111), obtém-se:

$$
\int_{\Gamma_{q}} q_{L} w_{3 \beta}^{*} d \Gamma_{q}=\frac{1}{2 \pi}\left\{q_{L}^{k_{1}}\left[n_{, \beta}+r_{, \beta}\left(-2+\ln l-\lim _{\varepsilon \rightarrow 0} \ln \varepsilon\right)\right]+q_{L}^{k_{2}}\left(2 r_{, \beta}\right)\right\}
$$

Considerando-se finalmente a contribuição da carga " $\mathrm{q}_{L}$ ", no cálculo das forças cortantes quando " $\xi$ " coincide com " $k_{3}$ ", conforme Fig. 3.13, chegam-se a equações análogas às (3.164) e (3.166), dadas por:

$$
\begin{aligned}
& \int_{\Gamma_{q}} q_{L} w_{\alpha \beta}^{*} d \Gamma_{q}=q_{L}^{k_{1}}\left\{\frac{(1+v) \delta_{\alpha \beta}}{4 \pi}\left[-\frac{2}{l^{2}} \int_{0}^{l} r^{2} \ln z d r+\frac{1}{l} \int_{0}^{l} r \ln z d r\right]+\right. \\
& \left.\frac{(1-v)\left(\delta_{\alpha \beta}-2 r_{, \alpha} r_{, \beta}\right)}{48 \pi} l+\frac{v\left(2 r_{, \alpha} r_{, \beta}-\delta_{\alpha \beta}\right)}{2 \pi \lambda^{2} l}\left(2-\ln l+\lim _{\varepsilon \rightarrow 0} \ln \varepsilon\right)\right\}+ \\
& q_{L}^{k_{2}}\left\{\frac{(1+v) \delta_{\alpha \beta}}{\pi}\left(\frac{1}{l^{2}} \int_{0}^{l} r^{2} \ln z d r-\frac{1}{l} \int_{0}^{l} r \ln z d r\right)+\frac{(1-v)\left(\delta_{\alpha \beta}-2 r_{, \alpha} r_{, \beta}\right)}{12 \pi} l+\right. \\
& \left.\frac{2 v\left(2 r_{, \alpha} r_{, \beta}-\delta_{\alpha \beta}\right)}{\pi \lambda^{2} l}\left(\ln l-\lim _{\varepsilon \rightarrow 0} \ln \varepsilon-1\right)\right\}+ \\
& q_{L}^{k_{3}}\left\{\frac{(1+v) \delta_{\alpha \beta}}{4 \pi}\left(-\frac{2}{l^{2}} \int_{0}^{l} r^{2} \ln z d r+\frac{3}{l} \int_{0}^{l} r \ln z d r-\int_{0}^{l} \ln z d r\right)+\right. \\
& \left.\frac{(1-v)\left(\delta_{\alpha \beta}-2 r_{, \alpha} r_{, \beta}\right)}{48 \pi} l+\frac{v\left(2 r_{, \alpha} r_{, \beta}-\delta_{\alpha \beta}\right)}{2 \pi \lambda^{2}}\left(\frac{1}{l}-\frac{3}{l} \ln l+\frac{3}{l} \lim _{\delta \rightarrow 0} \ln \varepsilon+\lim _{\varepsilon \rightarrow 0} \frac{1}{\varepsilon}\right)\right\}
\end{aligned}
$$




$$
\int_{\Gamma_{q}} q_{L} w_{3 \beta}^{*} d \Gamma_{q}=-\frac{1}{2 \pi}\left\{q_{L}^{k_{2}}\left(2 r_{, \beta}\right)+q_{L}^{k_{3}}\left[-n_{, \beta}+r_{, \beta}\left(-2+\ln l-\lim _{s \rightarrow 0} \ln \varepsilon\right)\right]\right\}
$$

Para se obter os coeficientes " $b_{i}^{\prime}(\xi)$ " ou " $b_{3+\beta}^{\prime}(\xi)$ " dados em (3.92), quando o ponto " $\xi$ " coincide com o ponto " $k_{1}{ }^{n}$ do elemento " $j$ " ou " $k_{3}$ " do elemento " $\mathrm{j}-1$ ", deve-se integrar numericamente sobre todos os elementos, excetuando-se os elementos " $\mathrm{j}-1$ " $\mathrm{e}$ " $\mathrm{j}$ ", onde a integração deve ser analítica,

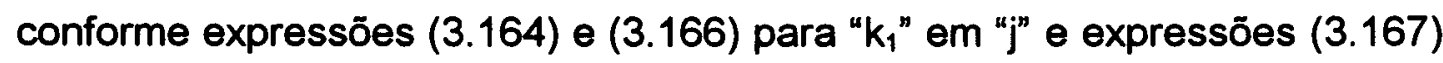
e (3.168), para " $k_{3}$ " em "j-1" e com isto os limites que aparecem nestas integrais se anulam, devido aos valores distintos de " $r, \alpha$ " nos dois elementos adjacentes.

A contribuiçăo da linha de momentos " $m_{\alpha}^{L}(x)^{n}$ no cálculo dos esforços em pontos internos, expressa em (3.65), será escrita de uma maneira discretizada como:

$$
\begin{aligned}
& b_{i}^{\prime}(\xi)=\sum_{j=1}^{N E C C} \frac{j}{2} \int_{-1}^{+1} u_{i \beta \gamma}^{*}(\xi, x) \underset{\sim m}{\psi_{\sim}^{T}}(\eta) d \eta \underset{\sim}{m_{\gamma}^{N}} \\
& b_{\beta+3}^{\prime}(\xi)=\sum_{j=1}^{N E C C} \frac{l_{j}}{2} \int_{-1}^{+1} u_{3 \beta r}^{*}(\xi, x) \underset{\sim m}{\psi_{\sim}^{T}}(\eta) d \eta \underset{\sim r}{m^{N}}
\end{aligned}
$$

onde " $\psi_{\sim}(\eta)$ " é dado em (3.92), " $u_{i \beta \gamma}^{*}$ "é dado em (3.29) e " $\underset{\sim}{\boldsymbol{m}_{\gamma} \text { " }}$ săo os valores nodais da linha de momentos " $m_{y}^{L}(x)^{n}$.

Os coeficientes (3.169) dão a contribuição da linha de momentos " $m_{\gamma}^{L}(x)$ ", no cálculo dos momentos $\left(b_{i}^{\prime}\right)$ e forças cortantes $\left(b_{\beta+3}^{\prime}\right)$ em pontos 
internos " $\xi$ ", e serão endereçados e armazenados convenientemente no vetor " $B^{\prime \prime}$ " em (3.153).

Caso o ponto " $\xi$ " coincida com um dos pontos nodais do elemento " $j$ ", os coeficientes (3.169) serão calculados analiticamente sobre este elemento. Será analisada primeiramente a contribuição da linha de momentos, quando o ponto " $\xi$ " coincide com o ponto nodal " $k_{2}$ " do elemento "j", conforme Fig. 3.11.

Segundo o item 3.2.5 a contribuiçăo da linha de momentos no cálculo dos momentos em pontos internos, quando o ponto " $\xi$ " coincide com o ponto " $k_{2}$ ", é dado na expressão (3.66), que transformada para a situação presente, ilustrada na Fig. 3.11, resulta:

$$
\int_{\Gamma_{m}} m_{\gamma}^{L} u_{\alpha \beta \gamma}^{*} d \Gamma_{m}=m_{\gamma}^{k_{2}} I_{\alpha \beta \gamma}+\int_{-1 / 2}^{0} m_{\gamma}^{L} u_{\alpha \beta \gamma}^{*} d \Gamma_{1}+\int_{0}^{1 / 2} m_{\gamma}^{L} u_{\alpha \beta \gamma}^{*} d \Gamma_{2}
$$

onde " $\mathrm{I}_{\alpha \beta \gamma}$ " representa $\mathrm{o}$ limite da integral para o contorno " $\Gamma_{\varepsilon}$ ", definido na Fig. 3.5 e apresentado em 3.69 , quando " $\varepsilon$ " tende a zero.

Analisando-se " $u_{\alpha \beta \gamma}^{*}$ ", expresso em (3.29), vê-se que a mesma é função entre outros de " $A$ " e " $K K_{1}$ ", definidos no apêndice $A$, e que portanto será necessário um esquema de integração analítica e numérica para cada trecho do elemento "j". Desta forma (3.170), a partir dos valores de " $r$ " dados em (3.98), pode ser escrita como:

$$
\int_{\Gamma_{m}} m_{\gamma}^{L} u_{\alpha \beta \gamma}^{*} d \Gamma_{m}=m_{\gamma}^{k_{2}} I_{\alpha \beta \gamma}+I_{A_{1}}+I_{N_{1}}+I_{A_{2}}+I_{N_{2}}
$$

onde " $I_{A_{\alpha}}$ " e " $I_{N_{\alpha}}$ ", são respectivamente as integrais analítica e numérica no trecho " $\alpha$ " do elemento "j", dadas por: 
120

$I_{A_{1}}=\int_{0}^{2 / \lambda} m_{\gamma}^{L} u_{\alpha \beta \gamma}^{*} d r_{1}$

$I_{N_{1}}=\int_{2 / \lambda}^{l / 2} m_{\gamma}^{L} u_{\alpha \beta \gamma}^{*} d r_{1}$

(3.172)

$$
\begin{aligned}
& I_{A_{2}}=\int_{0}^{2 / \lambda} m_{\gamma}^{L} u_{\alpha \beta \gamma}^{*} d r_{2} \\
& I_{N_{2}}=\int_{2 / \lambda}^{1 / 2} m_{\gamma}^{L} u_{\alpha \beta \gamma}^{*} d r_{2}
\end{aligned}
$$

Considerando-se " $m_{\gamma}^{L}(x)$ " dado em (3.92), com coeficientes " $\phi_{\alpha}$ " da matriz " $\psi_{m}(\eta)$ " dados segundo (3.103) para o trecho 1, e (3.104) para o trecho 2, pode-se escrever para a soma das integrais analíticas de (3.171):

$$
\begin{aligned}
& I_{A_{1}}+I_{A_{2}}=m_{r}^{k_{1}}\left[-\frac{2}{l}\left(C_{1} \int_{0}^{2 / \lambda} A d r+C_{2} \int_{0}^{2 / \lambda} z k_{1} d r+2 \frac{C_{3}}{\lambda}\right)\right]+ \\
& m_{\gamma}^{k_{3}}\left[\frac{2}{l}\left(C_{1} \int_{0}^{2 / \lambda} A d r+C_{2} \int_{0}^{2 / \lambda} z k_{1} d r+2 \frac{C_{3}}{\lambda}\right)\right]
\end{aligned}
$$

onde:

$$
\begin{aligned}
& C_{1}=\frac{r_{, \alpha} \delta_{\beta \gamma}+r_{, \beta} \delta_{\alpha \gamma}+r_{, \gamma} \delta_{\alpha \beta}-4 r_{, \alpha} r_{, \beta} r_{, \gamma}}{\pi} \\
& C_{2}=\frac{r_{, \alpha} \delta_{\beta \gamma}+r_{, \beta} \delta_{\alpha \gamma}-2 r_{, \alpha} r_{, \beta} r_{, \gamma}}{2 \pi} \\
& C_{3}=\frac{(1-v)\left(r_{, \alpha} \delta_{\beta \gamma}+r_{, \beta} \delta_{\alpha \gamma}-2 r_{, \alpha} r_{, \beta} r_{, \gamma}\right)+(1+v) r_{, \gamma} \delta_{\alpha \beta}}{4 \pi}
\end{aligned}
$$


com " $r, \alpha$ " dado em (3.105).

A integral com o termo " $A$ " é dada na expressão (3.125), e com o termo " $2 k_{1}$ ", conforme o apêndice $A$, pode ser escrita como:

$$
\int_{0}^{2 / \lambda}\left(z k_{1}\right) r^{n} d r=\left(\frac{2}{\lambda}\right)^{n+1}\left[1-0,5\left(\frac{2}{n+3}\right)^{2}-4 \sum_{i=2}^{12,2} \frac{e_{i} t^{* 1}}{(n+i+3)^{2}}+\sum_{i=2}^{12,2} \frac{f_{i}}{(n+i+1)}\right]
$$

onde:

$$
z k_{1}=z^{2} \ln \left(\frac{z}{2}\right)\left(0,5+\sum_{i=2}^{12,2} e_{i} t^{i}\right)+1+\sum_{i=2}^{12,2} f_{i}\left(\frac{z}{2}\right)^{i}
$$

com:

$$
\begin{aligned}
& e_{2}=0,87905940 \\
& e_{4}=0,51498869 \\
& e_{6}=0,15084934 \\
& e_{8}=0,02658733 \\
& e_{10}=0,00301532 \\
& e_{12}=0,00032411
\end{aligned}
$$

$f_{2}=0,15443144$

$f_{4}=-0,67278579$

$f_{6}=-0,18156897$

$f_{8}=-0,01919402$

$f_{10}=-0,00110404$

$f_{12}=-0,00004686$

t e t* são dados em (3.129). 
A integração numérica no trecho 1 , do elemento “j”, conforme (3.172) pode ser escrita como:

$$
I_{N_{1}}=\frac{\lambda l-4}{4 \lambda} \int_{-1}^{+1} m_{y}^{L} u_{\alpha \beta \gamma}^{*} d \eta_{1}
$$

onde " $\eta_{1}$ " é uma nova coordenada homogênea definida no trecho " $2 / \lambda \leq r_{1} \leq$ I/2", dada pela expressão (3.133). Os valores de " $m_{\gamma}^{L "} e$ " $u_{\alpha \beta \gamma}^{*}$ " são a partir de (3.133) funçōes da nova coordenada " $\eta_{1}$ ", podendo-se portanto integrar numericamente (3.178).

Analogamente, a integração numérica no trecho 2 resulta:

$$
I_{N_{2}}=\frac{\lambda l-4}{4 \lambda} \int_{-1}^{+1} m_{\gamma}^{L} u_{\alpha \beta \gamma}^{*} d \eta_{2}
$$

onde " $\eta_{2}$ " é dado em (3.134).

Para se obter portanto a contribuição da linha de momentos " $m_{\gamma}^{L}$ ", no cálculo dos momentos internos, quando o ponto fonte coincide com o nó " $k_{2}$ " do elemento "j", basta integrar numericamente sobre todos os elementos conforme (3.169), excetuando-se o elemento " $\mathrm{j}$ ", que terá duas parcelas de integração: uma analítica expressa em (3.173), e outra numérica, dada pela soma das expressões (3.178) e (3.179). Desta forma determinam-se os coeficientes " $b_{i}^{\prime}(\xi)$ ” em (3.169).

A determinação dos coeficientes " $b_{3+\beta}^{\prime}(\xi)$ ", que são a contribuição da

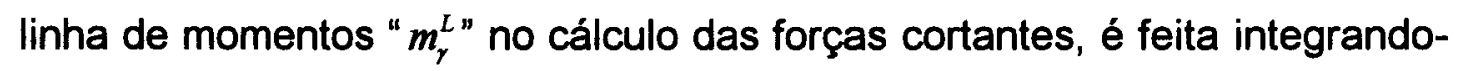
se numericamente sobre todos os elementos, conforme expresso na segunda equação de (3.169). Caso o ponto " $\xi$ " coincida com o nó "k", deve- 
se integrar analiticamente apenas para o elemento "j", que contém o nó singular " $\xi^{\prime}$. Segundo o item 3.2.5, o " $\lim _{s \rightarrow 0} \int_{\Gamma_{s}} m_{\gamma}^{L} u_{3 \beta \gamma}^{*} d \Gamma_{\varepsilon} "$ é igual a zero, podendo-se escrever:

$$
\int_{\Gamma_{m}} m_{r}^{L} u_{3 \beta \gamma}^{*} d \Gamma_{m}=\int_{-l / 2}^{0} m_{r}^{L} u_{3 \beta \gamma}^{*} d \Gamma_{1}+\int_{0}^{l / 2} m_{r}^{L} u_{3 \beta \gamma}^{*} d \Gamma_{2}
$$

Analisando-se a expressão de " $u_{3 \beta \gamma}^{*}$ " dada em (3.29), vê-se que esta solução fundamental é basicamente função de " $A$ " $e$ " $B$ ", valores definidos no apêndice $A$, sendo portanto necessário um esquema de integração analítica e numérica para cada trecho do elemento "j". Desta forma a partir de " $m_{\gamma}^{L}$ " dado em (3.92), com " $\phi_{i}$ " dado em (3.103) e (3.104), obtém-se:

$$
\begin{aligned}
& \int_{\Gamma_{m}} m_{r}^{L} u_{3 \beta \gamma}^{*} d \Gamma_{m}=\int_{0}^{2 / \lambda} m_{\gamma}^{L} u_{3 \beta \gamma}^{*} d r_{1}+\int_{2 / \lambda}^{l / 2} m_{r}^{L} u_{3 \beta \gamma}^{*} d r_{1}+ \\
& \int_{0}^{2 / \lambda} m_{r}^{L} u_{3 \beta \gamma}^{*} d r_{2}+\int_{2 / \lambda}^{l / 2} m_{\gamma}^{L} u_{3 \beta \gamma}^{*} d r_{2}
\end{aligned}
$$

Integrando-se analiticamente e somando-se a primeira e terceira parcelas do segundo membro de (3.181) obtém-se:

$$
\begin{gathered}
\int_{0}^{2 / \lambda} m_{\gamma}^{L} u_{3 \beta \gamma}^{*} d r_{1}+\int_{0}^{2 / \lambda} m_{\gamma}^{L} u_{3 \beta r}^{*} d r_{2}=\frac{\lambda^{2}}{\pi}\left\{m_{\gamma}^{k_{1}}\left[\frac{2}{l^{2}}\left(\delta_{\beta \gamma} \int_{0}^{2 / \lambda} B r^{2} d r-r_{, \beta} r_{, \gamma} \int_{0}^{2 / \lambda} A r^{2} d r\right)\right]+\right. \\
m_{\gamma}^{k_{2}}\left[\delta_{\beta \gamma}\left(\int_{0}^{2 / \lambda} B d r-\frac{4}{l^{2}} \int_{0}^{2 / \lambda} B r^{2} d r\right)-r_{, \beta} r_{, \gamma}\left(\int_{0}^{2 / \lambda} A d r-\frac{4}{l^{2}} \int_{0}^{2 / \lambda} A r^{2} d r\right)\right]+ \\
\left.m_{\gamma}^{k_{3}}\left[\frac{2}{l^{2}}\left(\delta_{\beta \gamma} \int_{0}^{2 / \lambda} B r^{2} d r-r_{, \beta} r_{, r} \int_{0}^{2 / \lambda} A r^{2} d r\right)\right]\right\}
\end{gathered}
$$

onde as integrais contendo " $A$ " $\mathrm{e}$ " $B$ " são apresentadas respectivamente nas expressões (3.125) e (3.126), e "r,a" é dado em (3.105). 
A segunda e quarta parcelas de (3.181) são integradas numericamente, resultando:

$$
\begin{aligned}
& \int_{2 / \lambda}^{l / 2} m_{\gamma}^{L} u_{3 \beta \gamma}^{*} d r_{1}+\int_{2 / \lambda}^{l / 2} m_{\gamma}^{L} u_{3 \beta \gamma}^{*} d r_{2}=\frac{\lambda l-4}{4 \lambda} \int_{-1}^{+1} m_{\gamma}^{L} u_{3 \beta \gamma}^{*} d \eta_{1}+ \\
& \frac{\lambda l-4}{4 \lambda} \int_{-1}^{+1} m_{\gamma}^{L} u_{3 \beta \gamma}^{*} d \eta_{2}
\end{aligned}
$$

onde " $\eta_{1}$ " $e$ " $\eta_{2}$ " são novas coordenadas homogêneas definidas nos trechos 1 e 2, representadas respectivamente em (3.133) e (3.134).

Será analisado agora a situação em que o ponto " $\xi$ " coincide com o nó "k" do elemento "j", conforme Fig. 3.12. Inicialmente considera-se a influência da linha de momentos no cálculo dos momentos internos, ficando a expressão (3.170) para este caso, dada por:

$$
\int_{\Gamma_{m}} m_{\gamma}^{L} u_{\alpha \beta \gamma}^{*} d \Gamma_{m}=m_{r}^{k_{1}} I_{\alpha \beta \gamma}+\int_{0}^{2 / \lambda} m_{r}^{L} u_{\alpha \beta \gamma}^{*} d r+\int_{2 / \lambda}^{l} m_{\gamma}^{L} u_{\alpha \beta \gamma}^{*} d r
$$

com "I $\mathrm{I}_{\alpha \beta \gamma}$ " definido em (3.69), sendo a primeira integral do segundo membro calculada analiticamente e a segunda numericamente.

Para " $m_{\gamma}^{L "}$ expresso em (3.92) com suas componentes " $\phi_{i}$ " dadas em (3.111), obtém-se:

$$
\begin{aligned}
& \int_{0}^{2 / \lambda} m_{r}^{L} u_{\alpha \beta \gamma}^{*} d r=m_{r}^{k_{1}}\left[C_{1}\left(\frac{2}{l^{2}} \int_{0}^{2 / \lambda} A r d r-\frac{3}{l} \int_{0}^{2 / \lambda} A d r+\int_{0}^{2 / \lambda} \frac{A}{r} d r\right)+\right. \\
& C_{2}\left(\frac{2}{l^{2}} \int_{0}^{2 / \lambda} z k_{1} r d r-\frac{3}{l} \int_{0}^{2 / \lambda} z k_{1} d r+\int_{0}^{2 / \lambda} \frac{z k_{1}}{r} d r\right)+ \\
& \left.C_{3}\left(\frac{4}{\lambda^{2} l^{2}}-\frac{6}{\lambda l}+\ln \frac{2}{\lambda}-\lim _{\varepsilon \rightarrow 0} \ln \varepsilon\right)\right]+
\end{aligned}
$$




$$
\begin{aligned}
& m_{\gamma}^{k_{2}}\left[C_{1}\left(\frac{4}{l} \int_{0}^{2 / \lambda} A d r-\frac{4}{l^{2}} \int_{0}^{2 / \lambda} A r d r\right)+C_{2}\left(\frac{4}{l} \int_{0}^{2 / \lambda} z k_{1} d r-\frac{4}{l^{2}} \int_{0}^{2 / \lambda} z k_{1} r d r\right)+\right. \\
& \left.C_{3}\left(\frac{8}{\lambda l}-\frac{8}{\lambda^{2} l^{2}}\right)\right]+m_{\gamma}^{k_{3}}\left[C_{1}\left(\frac{2}{l^{2}} \int_{0}^{2 / \lambda} A r d r-\frac{1}{l} \int_{0}^{2 / \lambda} A d r\right)+\right. \\
& \left.C_{2}\left(\frac{2}{l^{2}} \int_{0}^{2 / \lambda} z k_{1} r d r-\frac{1}{l} \int_{0}^{2 / \lambda} z k_{1} d r\right)+C_{3}\left(\frac{4}{\lambda^{2} l^{2}}-\frac{2}{\lambda l}\right)\right]
\end{aligned}
$$

onde " $C_{1}$ ", " $\mathrm{C}_{2}$ " e " $\mathrm{C}_{3}$ " estăo apresentados em (3.174); as integrais envolvendo " $A r^{n "},{ }^{~ " B r} r^{n "}$ e " $z k_{1} r^{n ",}$, para $n \geq 0$, estão apresentadas respectivamente em (3.125), (3.126) e (3.175). As integrais envolvendo " $A / r$ " e " $z k_{1} / r$ " são dadas por:

$$
\int_{0}^{2 / \lambda} \frac{A}{r} d r=-\sum_{i=2}^{12,2} \frac{a_{i} t^{* 1}}{i^{2}}+\sum_{i=2}^{12,2} \frac{b_{i}}{i}-0,5\left[\ln \left(\frac{2}{\lambda}\right)-\lim _{\varepsilon \rightarrow 0} \ln \varepsilon\right]
$$

com " $t^{*}=\frac{2}{3,75}$ " " $a_{i}$ " e " $b_{i}$ " expressos em (3.130).

$$
\int_{0}^{2 / \lambda} \frac{z k_{1}}{r} d r=-0,5+\ln \frac{2}{\lambda}-\lim _{\delta \rightarrow 0} \ln \varepsilon-4 \sum_{i=2}^{12,2} \frac{e_{i} t^{* t}}{(i+2)^{2}}+\sum_{i=2}^{12,2} \frac{f_{i}}{i}
$$

com " $e_{i}$ " $e$ " $f_{i}$ " dados em (3.177).

A terceira parcela do segundo membro de (3.184), integrada numericamente resulta:

$$
\int_{2 / \lambda}^{l / 2} m_{r}^{L} u_{\alpha \beta \gamma}^{*} d r=\frac{\lambda l-2}{2 \lambda} \int_{-1}^{+1} m_{r}^{L} u_{\alpha \beta \gamma}^{*} d \eta_{1}
$$

com " $\eta_{1}$ " dado em (3.141). 
A contribuição da linha de momentos, no cálculo das forças cortantes, quando " $\xi$ " coincide com " $k_{1}$ ", é obtida analogamente à expressão (3.181), resultando:

$$
\int_{\Gamma_{*}} m_{\gamma}^{L} u_{3 \beta \gamma}^{*} d \Gamma_{m}=\int_{0}^{2 / \lambda} m_{r}^{L} u_{3 \beta \gamma}^{*} d r+\int_{2 / \lambda}^{l} m_{r}^{L} u_{3 \beta \gamma}^{*} d r
$$

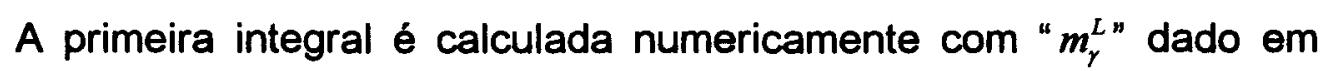
(3.92) para " $\phi_{i}$ " expressos em (3.111). Assim:

$$
\begin{aligned}
& \int_{0}^{2 / \lambda} m_{r}^{L} u_{3 \beta_{r}}^{*} d r=\frac{\lambda^{2}}{2 \pi}\left\{m _ { r } ^ { k _ { r } } \left[\delta_{\beta r}\left(\frac{2}{l^{2}} \int_{0}^{2 / \lambda} B r^{2} d r-\frac{3}{l} \int_{0}^{2 / \lambda} B r d r+\int_{0}^{2 / \lambda} B d r\right)-\right.\right. \\
& \left.r_{, \beta} r_{, r}\left(\frac{2}{l^{2}} \int_{0}^{2 / \lambda} A r^{2} d r-\frac{3}{l} \int_{0}^{2 / \lambda} A r d r+\int_{0}^{2 / \lambda} A d r\right)\right]+ \\
& m_{r}^{k_{2}}\left[\delta_{\beta r}\left(\frac{4}{l} \int_{0}^{2 / \lambda} B r d r-\frac{4}{l^{2}} \int_{0}^{2 / \lambda} B r^{2} d r\right)-\right. \\
& \left.r_{, \beta} r_{,}\left(\frac{4}{l} \int_{0}^{2 / \lambda} A r d r-\frac{4}{l^{2}} \int_{0}^{2 / \lambda} A r^{2} d r\right)\right]+ \\
& m_{r}^{k_{3}}\left[\delta_{\beta_{r}}\left(\frac{2}{l^{2}} \int_{0}^{2 / \lambda} B r^{2} d r-\frac{1}{l} \int_{0}^{2 / \lambda} B r d r\right)-\right. \\
& \left.r_{, \beta} r_{, r}\left(\frac{2}{l^{2}} \int_{0}^{2 / \lambda} A r^{2} d r-\frac{1}{l} \int_{0}^{2 / \lambda} A r d r\right)\right]
\end{aligned}
$$

A segunda integral de (3.189) é calculada numericamente, obtendose:

$$
\int_{2 / \lambda}^{l / 2} m_{\gamma}^{L} u_{3 \beta \gamma}^{*} d r=\frac{\lambda l-2}{2 \lambda} \int_{-1}^{+1} m_{\gamma}^{L} u_{3 \beta \gamma}^{*} d \eta_{1}
$$

com " $\eta_{1}$ " dado em (3.141) e "r,a" em (3.105). 
127

Finalmente será analisada a contribuição da linha de momentos, no

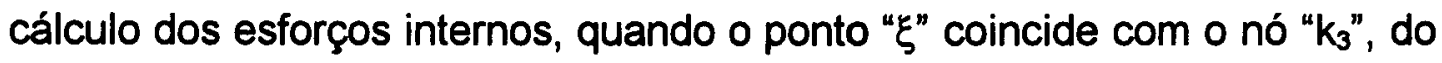
elemento "j". Assim, considerando-se o cálculo dos momentos internos, pode-se escrever a expressão (3.184) para esta nova situação como:

$$
\int_{\Gamma_{m}} m_{\gamma}^{L} u_{\alpha \beta \gamma}^{*} d \Gamma_{m}=m_{r}^{k_{3}} I_{\alpha \beta \gamma}+\int_{0}^{2 / \lambda} m_{y}^{L} u_{\alpha \beta \gamma}^{*} d r+\int_{2 / \lambda}^{l / 2} m_{\gamma}^{L} u_{\alpha \beta \gamma}^{*} d r
$$

onde "I $\alpha \beta \gamma$ " está expresso em (3.69).

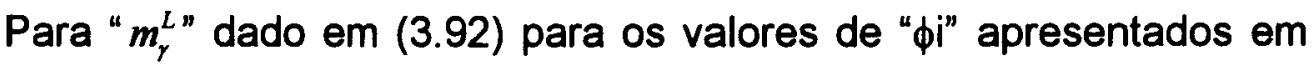
(3.115), obtém-se para a primeira integral do segundo membro de (3.192), que será integrada analiticamente:

$$
\begin{aligned}
& \int_{0}^{2 / \lambda} m_{r}^{L} u_{\alpha \beta r}^{*} d r=m_{r}^{k_{1}}\left[C_{1}\left(\frac{2}{l^{2}} \int_{0}^{2 / \lambda} A r d r-\frac{1}{l} \int_{0}^{2 / \lambda} A d r\right)+\right. \\
& \left.C_{2}\left(\frac{2}{l^{2}} \int_{0}^{2 / \lambda} z k_{1} r d r-\frac{1}{l} \int_{0}^{2 / \lambda} z k_{1} d r\right)+C_{3}\left(\frac{4}{\lambda^{2} l^{2}}-\frac{2}{\lambda l}\right)\right]+ \\
& m_{r}^{k_{2}}\left[C_{1}\left(\frac{4}{l} \int_{0}^{2 / \lambda} A d r-\frac{4}{l^{2}} \int_{0}^{2 / \lambda} A r d r\right)+C_{2}\left(\frac{4}{l} \int_{0}^{2 / \lambda} z k_{1} d r-\frac{4}{l^{2}} \int_{0}^{2 / \lambda} z k_{1} r d r\right)+\right. \\
& \left.C_{3}\left(\frac{8}{\lambda l}-\frac{8}{\lambda^{2} l^{2}}\right)\right]+m_{r}^{k_{3}}\left[C_{1}\left(\frac{2}{l^{2}} \int_{0}^{2 / \lambda} A r d r-\frac{3}{l} \int_{0}^{2 / \lambda} A d r+\int_{0}^{2 / \lambda} \frac{A}{r} d r\right)+\right. \\
& C_{2}\left(\frac{2}{l^{2}} \int_{0}^{2 / \lambda} z k_{1} r d r-\frac{3}{l} \int_{0}^{2 / \lambda} z k_{1} d r+\int_{0}^{2 / \lambda} \frac{z k_{1}}{r} d r\right)+ \\
& \left.C_{3}\left(\frac{4}{\lambda^{2} l^{2}}-\frac{6}{\lambda l}+\ln \frac{2}{\lambda}-\lim _{\varepsilon \rightarrow 0} \ln \varepsilon\right)\right]
\end{aligned}
$$

onde " $\mathrm{C}_{1}$ ", " $\mathrm{C}_{2}$ " e " $\mathrm{C}_{3}$ " são dados em (3.174), as integrais envolvendo " $\mathrm{Ar}$ " e " $B r^{n "}$ estão dadas respectivamente em (3.125) e (3.126), as integrais 
envolvendo " $z k_{1} r^{n_{n}}$ estão dadas em (3.175), as integrais envolvendo " $A r$ " e " $z k_{1} / r$ " estão dadas respectivamente em (3.186) e (3.187).

A integral numérica em (3.192) fica:

$$
\int_{2 / \lambda}^{1 / 2} m_{r}^{L} u_{\alpha \beta \gamma}^{*} d r=\frac{\lambda l-2}{2 \lambda} \int_{-1}^{+1} m_{r}^{L} u_{\alpha \beta \gamma}^{*} d \eta_{1}
$$

com " $\eta_{1}$ " dado em (3.146).

Para o cálculo das forças cortantes quando " $\xi$ " coincide com " $\mathrm{k}_{3}$ ", a contribuição da linha de momentos é dada pela mesma expressão (3.189), lembrando-se que na situação presente a linha de momentos é dada em (3.92), com os coeficientes " $\phi_{i}$ " expressos em (3.115). Assim a integral analítica fica:

$$
\begin{aligned}
& \int_{0}^{2 / \lambda} m_{r}^{L} u_{3 \beta r}^{*} d r=\frac{\lambda^{2}}{2 \pi}\left\{m _ { r } ^ { k _ { 1 } } \left[\delta_{\beta r}\left(\frac{2}{l^{2}} \int_{0}^{2 / \lambda} B r^{2} d r-\frac{1}{l} \int_{0}^{2 / \lambda} B r d r\right)-\right.\right. \\
& \left.r_{, \beta} r_{, r}\left(\frac{2}{l^{2}} \int_{0}^{2 / \lambda} A r^{2} d r-\frac{1}{l} \int_{0}^{2 / \lambda} A r d r\right)\right]+ \\
& m_{r}^{k_{2}}\left[\delta_{\beta r}\left(\frac{4}{l} \int_{0}^{2 / \lambda} B r d r-\frac{4}{l^{2}} \int_{0}^{2 / \lambda} B r^{2} d r\right)-r_{, \beta} r_{, r}\left(\frac{4}{l} \int_{0}^{2 / \lambda} A r d r-\frac{4}{l^{2}} \int_{0}^{2 / \lambda} A r^{2} d r\right)\right]+ \\
& m_{r}^{k_{3}}\left[\delta_{\beta r}\left(\frac{2}{l^{2}} \int_{0}^{2 / \lambda} B r^{2} d r-\frac{3}{l} \int_{0}^{2 / \lambda} B r d r+\int_{0}^{2 / \lambda} B d r\right)-\right. \\
& \left.\left.r_{, \beta} r_{, r}\left(\frac{2}{l^{2}} \int_{0}^{2 / \lambda} A r^{2} d r-\frac{3}{l} \int_{0}^{2 / \lambda} A r d r+\int_{0}^{2 / \lambda} A d r\right)\right]\right\}
\end{aligned}
$$

onde " $r_{, \beta}$ " está dado em (3.105) e as integrais envolvendo " $A$ " e " $B$ " estão representadas respectivamente em (3.125) e (3.126). 
A parcela de (3.189) para este caso, com integração numérica, resulta:

$$
\int_{2 / \lambda}^{l / 2} m_{\gamma}^{L} u_{3 \beta \gamma}^{*} d r=\frac{\lambda l-2}{2 \lambda} \int_{-1}^{+1} m_{\gamma}^{L} u_{3 \beta \gamma}^{*} d \eta_{1}
$$

onde " $\eta_{1}$ " é dado em (3.146).

\subsection{6 - TÉCNICA DE SOLUÇÃO}

No item 3.3.2, a equação integral de contorno para placas pela Teoria de Reissner, foi discretizada dando origem a um sistema de equaçōes algébricas lineares, expresso matricialmente por:

$$
\underset{\sim}{H} \underset{\sim}{U}=\underset{\sim}{G} \underset{\sim}{P}+\underset{\sim}{B}
$$

A solução da equação matricial (3.86) para um problema real bem condicionado, com um número suficiente de deslocamentos e forças prescritos no contorno, se dará realizando-se algumas operações matriciais. Desta forma, visando-se agrupar todas as incógnitas do contorno em um único vetor, trocam-se as colunas correspondentes à deslocamentos prescritos, entre as matrizes " $H^{\prime}$ " e "G" ${ }_{\sim}$ ", alterando-se (3.86) para:

$$
\underset{\sim}{A} \underset{\sim}{X}=\underset{\sim}{F}
$$

onde: 
$\underset{\sim}{X} \quad$ é o vetor das incógnitas do contorno

$\stackrel{A}{\sim}$ - é a matriz contendo os coeficientes das incógnitas, obtida pela troca de colunas entre " $\underset{\sim}{H}$ " $e$ "G ${ }_{\sim}$. Em geral esta matriz é bem condicionada com termos diagonais dominantes, não-simétrica $e$ cheia.

$\underset{\sim}{F} \quad$ - é o vetor contendo a influência dos valores prescritos de contorno, mais o vetor " $B$ " correspondente a influência de todos os carregamentos de domínio.

Multiplicando-se ambos os membros de (3.197) por " ${ }_{\sim}{ }^{-1 \text {, }}$, obtém-se a solução do sistema, dada por:

$$
\underset{\sim}{X}=\underset{\sim}{A^{-1}} \underset{\sim}{F}
$$

0 vetor " $X$ " em (3.198) contém deslocamentos e forças de superfície, que endereçados convenientemente, vão formar juntamente com os valores de contorno prescritos, os vetores finais " $U^{n}$ e " $P_{\sim}$, , agora com todos os valores conhecidos.

A partir de " $U^{n}$ e " $P^{\prime}$ " determinam-se os deslocamentos e esforços em pontos internos dados respectivamente por:

$$
\begin{aligned}
& U_{\sim}=\underset{\sim}{G_{i}} \underset{\sim}{P}-\underset{\sim}{H_{i}} \underset{\sim}{U}+\stackrel{\sim}{B}^{B_{i}} \\
& \underset{\sim}{E}=\underset{\sim}{G^{\prime}} \underset{\sim}{P}-{\underset{\sim}{H}}_{\sim}^{U}+{\underset{\sim}{B}}^{\prime}
\end{aligned}
$$




\section{ANÁLISE DE PLACAS COM ENRIJECEDORES}

\section{1- INTRODUÇÃO}

Conforme visto anteriormente, o Método dos Elementos de Contorno, aplicado à análise de placas, conduz a um sistema de equações algébricas lineares, representado matricialmente pela equação (3.86), cujas incógnitas são os deslocamentos e forças de superfície no contorno. Este sistema não permite a análise de placas com vinculações internas, como por exemplo lajes cogumelo e lajes com vigamento interno, muito comum nos projetos de edifícios.

Neste capitulo a equação (3.86) será modificada para prever a presença de pontos ou linhas internas, onde os deslocamentos ou esforços serão prescritos, permitindo-se portanto a análise das placas referidas acima. Esta modificação introduzirá novas incógnitas, sendo necessário a obtenção de novas equações para que o sistema possa ser resolvido. Estas novas equações são obtidas escrevendo-se equaçōes integrais de deslocamentos, para cada ponto onde houver vinculação interna.

Inicialmente será apresentada uma formulação envolvendo vinculação proveniente de apoios internos pontuais ou distribuído em pequenas áreas, análogo ao desenvolvido no trabalho de PAIVA[113]. Posteriormente a formulação será estendida para apoios em linhas, sendo que o enrijecimento produzido pela estrutura formada de barras, será obtido através do Método dos Elementos Finitos. 


\section{2 - PLACAS SOBRE APOIOS DISCRETOS}

\subsection{1 - APOIOS PONTUAIS}

O vetor " $B$ " da equação matricial (3.86), armazena a influência de todo o carregamento externo aplicado à placa. Este carregamento, conforme visto no capítulo 3, pode estar atuando em áreas internas, pontos ou linhas. Este carregamento prescrito, pode também ser interpretado como reações incógnitas da interface placa-viga ou placa-pilar. No último caso, quando a área de contato é pequena em comparação com a área da placa, pode-se admití-la pontual, sendo as reações interpretadas como cargas concentradas, aplicadas à placa no ponto de contato "j", com valor desconhecido " $R_{j}$ ", conforme Fig. 4.1.
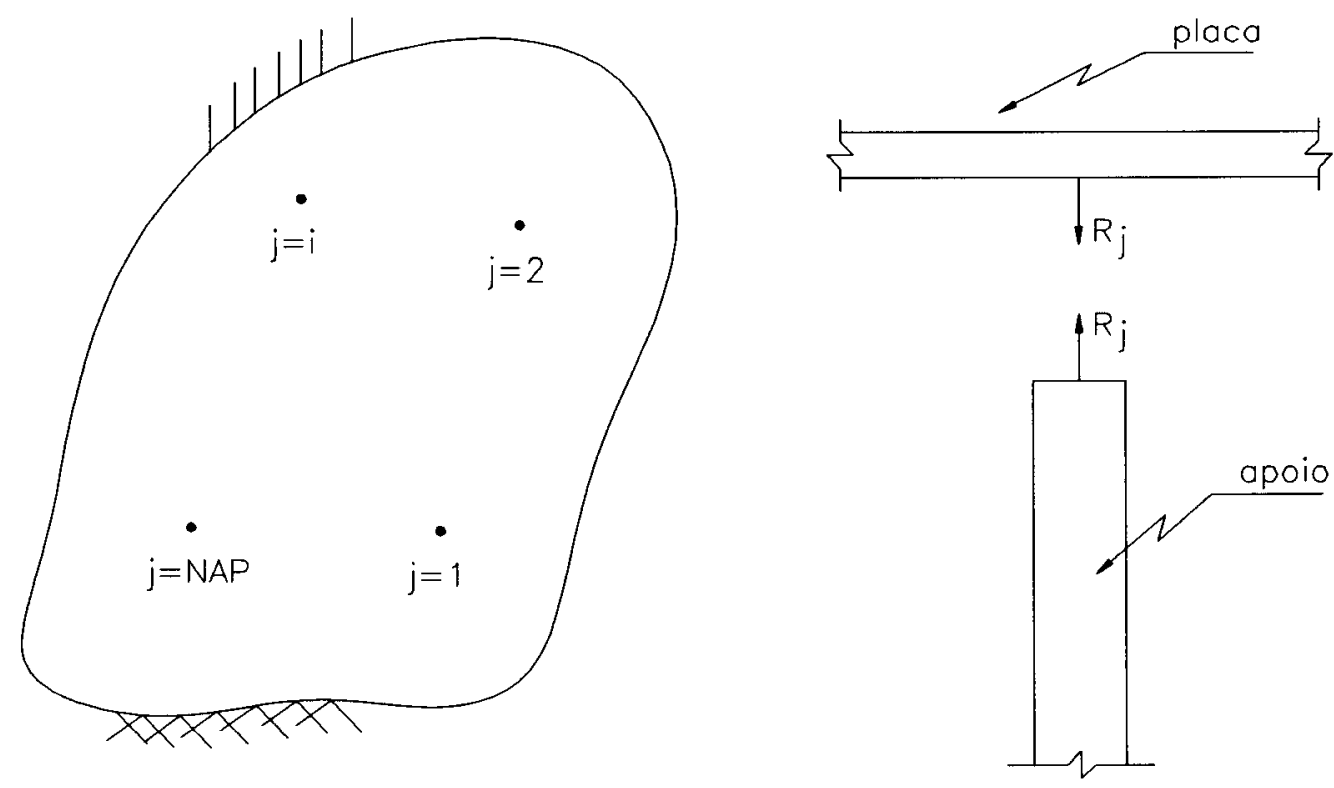

Fig. 4.1 - Placa sobre apoios pontuais. 
Considerando-se uma placa com "NAP", apoios internos, a equação (3.86) pode ser reescrita como:

$$
\underset{\sim}{H} \underset{\sim}{U}=\underset{\sim}{G} \underset{\sim}{P}+\underset{\sim}{B}+\underset{\sim}{S} \underset{\sim}{R}
$$

onde " $R$ " é o vetor das reações com 3(NAP) elementos e " $S$ " é uma matriz 3(NNCP) x3(NAP) contendo a influência dos termos:

$$
\begin{aligned}
& S_{\alpha \beta}=u_{\alpha \beta}^{*}(\chi, x) \\
& S_{3 \alpha}=u_{3 \alpha}^{*}(\chi, x) \\
& S_{i 3}=u_{i 3}^{*}(\chi, x)-\frac{v}{(1-v) \lambda^{2}} u_{i \alpha, \alpha}^{*}(\chi, x)
\end{aligned}
$$

As soluções fundamentais " $u_{i j}^{*}$ " são dadas em (2.32) e " $u_{i \alpha, \alpha}^{*}$ " é dada em (3.21).

Para cada apoio interno tem-se três reações, as duas primeiras correspondem respectivamente aos momentos " $R_{M_{x 1}}$ " e " $R_{M_{x 2}}$ ", a terceira representa a reação vertical " $R_{w}$ ".

Para deslocamentos prescritos nos "NAP" apoios internos, tem-se na equação (4.1), 3(NAP) reaçōes incógnitas, necessitando-se portanto de mais 3(NAP) novas equações, para que o sistema seja resolvido. Estas novas equações são obtidas, escrevendo-se equações integrais referentes aos deslocamentos internos ( $\phi_{x_{1}}, \phi_{x_{2}}$ e w) para os "NAP" apoios internos. Segundo a equação matricial (3.95) estas novas equações podem ser agrupadas e escritas como: 


$$
\underset{\sim}{U_{i}}=\underset{\sim}{G_{i}} \underset{\sim}{P}-\underset{\sim}{H_{i}} \underset{\sim}{U}+\underset{\sim}{B_{i}}+\underset{\sim}{S_{i} R}
$$

onde " $S_{i}$ " é obtido de maneira análoga a " $\underset{\sim}{S}$ ", tomando-se precauções com as indeterminações, quando " $\xi$ " coincide com " $x$ ".

A expressão (4.3) pode ser reescrita como:

$$
\left[\begin{array}{cc}
H_{i} & I \\
\sim
\end{array}\right]\left\{\begin{array}{c}
U \\
\underset{\sim}{U} \\
\sim
\end{array}\right\}=\underset{\sim}{G} \underset{\sim}{P}+\underset{\sim}{B_{i}}+\underset{\sim}{S_{i} R}
$$

Agrupando-se (4.1) e (4.4) obtém-se:

$$
\left[\begin{array}{cc}
\underset{H}{H} & 0 \\
\tilde{H}_{i} & \tilde{I} \\
\sim
\end{array}\right]\left\{\begin{array}{c}
U \\
\tilde{U}_{i} \\
\sim
\end{array}\right\}=\left[\begin{array}{c}
G \\
\tilde{G} \\
\mathcal{G}_{i}
\end{array}\right] \underset{\sim}{P}+\left[\begin{array}{c}
B \\
\tilde{B}_{i} \\
\sim
\end{array}\right]+\left[\begin{array}{c}
S \\
\tilde{S} \\
\underset{\sim}{S}
\end{array}\right] \underset{\sim}{R}
$$

Caso " $R$ " seja prescrito, por exemplo, cargas externas concentradas, " $U_{i}$ " é incógnito, resultando (4.5):

$$
\left[\begin{array}{cc}
H & 0 \\
\tilde{H}_{i} & \tilde{I} \\
\sim & \sim
\end{array}\right]\left\{\begin{array}{c}
U \\
\tilde{U}_{i} \\
\sim
\end{array}\right\}=\left[\begin{array}{cc}
G & \underset{S}{\tilde{G}} \\
\tilde{S} \\
\sim \sim & \sim
\end{array}\right]\left\{\begin{array}{c}
P \\
\tilde{R} \\
R
\end{array}\right\}+\left[\begin{array}{c}
B \\
\tilde{B_{i}} \\
\sim
\end{array}\right]
$$

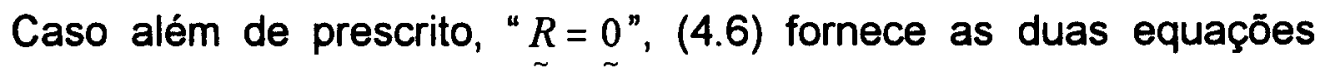
matriciais para deslocamentos em pontos do contorno (3.86), e em pontos do domínio (3.95).

Para valores de " $U_{i}$ " prescritos, (4.6) transforma-se em:

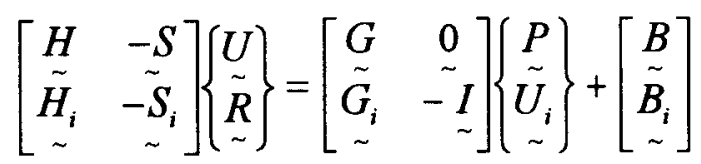


Impondo-se as condiçōes de contorno, o sistema (4.7) é resolvido, determinando-se além das incógnitas do contorno, os valores das reações internas " $R$ ". Com estas últimas, determinam-se os deslocamentos e esforços em pontos internos através das equações (3.95) e (3.153) respectivamente, onde os vetores " $B_{i}{ }^{n} \mathrm{e}^{\text {" }} \boldsymbol{B}^{\prime}$ " conterăo também a influência destas reaçōes.

Supondo-se que os apoios " $\mathrm{j}$ " sejam as ligaçōes da placa com pilares e que os mesmos tenham rigidez axial infinita, e rigidez a flexăo nas direções " $x_{1}$ " $e$ " $x_{2}$ " nulas, os deslocamentos " $W_{j}$ " $e$ as reações " $R_{M_{x 1}}$ " $e$ ${ }^{\text {" }} \mathbf{R}_{\mathrm{M}_{x}}$ " serăo nulas. Esta é a situação em geral utilizada na análise de lajes cogumelos, onde as incógnitas que interessam são as reações verticais. Para esta análise basta apenas escrever as equaçōes correspondentes aos deslocamentos transversais " $W_{j}$ " para os "NAP" apoios internos, ficando a equação (4.7) reescrita como:

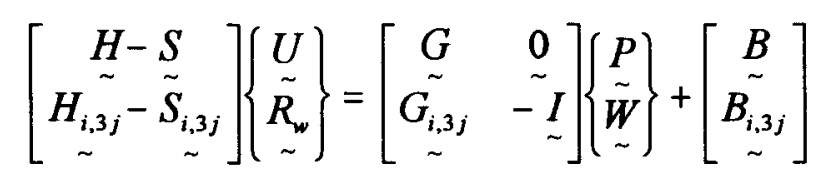

Resolvido o sistema (4.8) os deslocamentos e esforços em pontos internos serăo obtidos respectivamente pelas equações (3.95) e (3.153), onde os vetores " $B_{i}$ " e " $\underline{\sim}^{\prime \prime}$ " conterão além da influência das cargas de domínios, as reações " $R_{w}$ " dos "NAP" apoios internos.

\subsection{2 - APOIOS EM ÁREAS DISCRETAS}

Considera-se neste item a associação placa-pilar, onde a interface é constituída por uma área. Como hipótese admite-se que a área de interface 
permaneça plana após a deformação do conjunto, o que implica numa distribuição linear de tensões na interface.

Seja a Fig. 4.2, onde mostra-se uma área genérica da interface placapilar, indicando-se o sistema de referência global " $x_{1} x_{2} x_{3}$ " e o sistema local do pilar " $\bar{x} \bar{y} \bar{z} "$.

A tensão na área de interface pode ser dada pela expressão seguinte:

$$
\sigma(x)=\frac{R_{w}}{S}-\frac{R_{M_{\bar{y}}}}{I_{\bar{y} \bar{y}}} \bar{x}+\frac{R_{M_{\bar{z}}}}{I_{\bar{x} \bar{x}}} \bar{y}
$$

onde o sistema de coordenadas " $\bar{x} \bar{y}$ " representa os eixos principais de inércia da área da interface. Este sistema relaciona-se com o sistema " $x_{1} x_{2}$ " da seguinte forma:

$$
\left\{\begin{array}{l}
x_{1}(x)=x_{0}+\bar{x}(x) \\
x_{2}(x)=y_{0}+\bar{y}(x)
\end{array}\right.
$$

onde " $\mathrm{x}_{0}$ " $\mathrm{e}$ " $\mathrm{Y}_{0}$ " são as coordenadas do centro da interface.

Segundo a Fig. 4.2.c pode-se escrever:

$$
\left\{\begin{array}{l}
x_{1}(x)=x_{1}(\xi)+r \cos \theta \\
x_{2}(x)=x_{2}(\xi)+r \operatorname{sen} \theta
\end{array}\right.
$$

Levando-se (4.11) e (4.10) em (4.9) obtém-se:

$$
\sigma(x)=\sigma(\xi)-\left(A_{1} \cos \theta+B_{1} \operatorname{sen} \theta\right) r
$$

onde:

$$
A_{1}=\frac{M_{1}}{I_{\overline{y y}}}=\frac{R_{M_{\bar{y}}}}{I_{\overline{y y}}}
$$




$$
B_{1}=\frac{M_{2}}{I_{\bar{x} \bar{x}}}=-\frac{R_{M_{\bar{z}}}}{I_{\bar{x} \bar{x}}}
$$

$$
\sigma(\xi)=\frac{R_{w}}{S}+A_{1}\left[x_{0}-x_{1}(\xi)\right]+B_{1}\left[y_{0}-x_{2}(\xi)\right]
$$

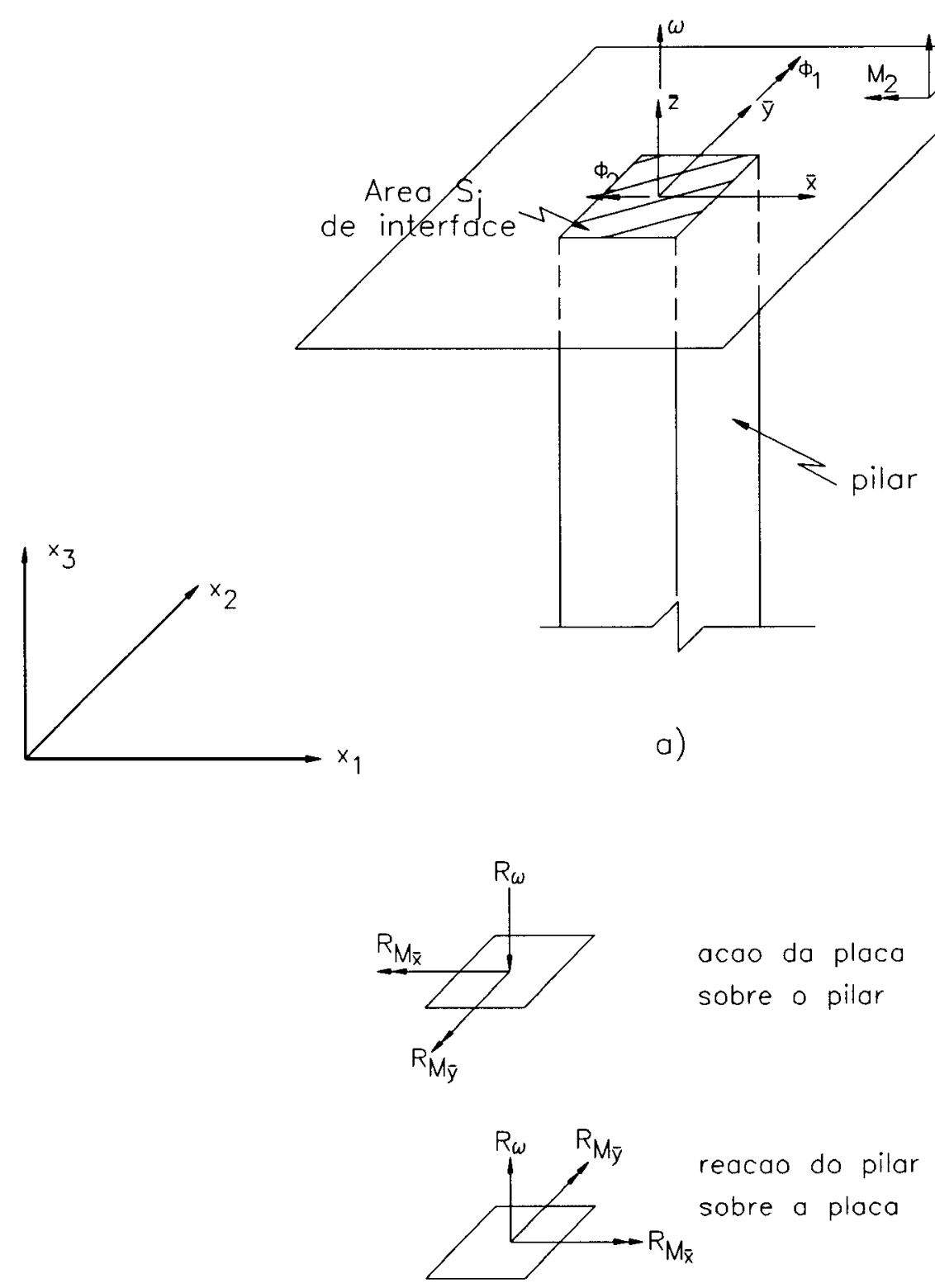

b) 


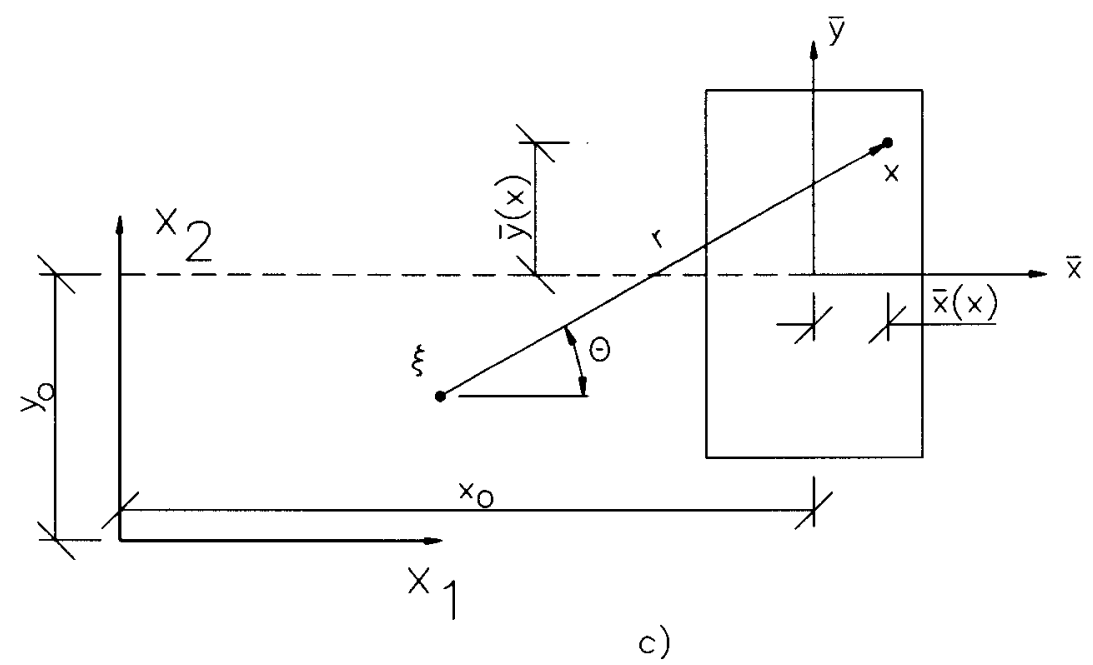

Fig. 4.2 -Associação placa-pilar : a) apoio genérico

b) reações na interface

c) sistemas de referência

A equação (4.12) representa a distribuição de tensões na área " $S_{j}$ " da interface placa-pilar, mas pode ser interpretada como uma carga distribuída linearmente, aplicada na mesma área, conforme a alternativa de carregamento dada no item 3.2.5, pela equação (3.42.b). Desta forma, a contribuição deste carregamento linear expresso em (4.12) no cálculo dos deslocamentos é dada pela equação (3.44).

Seja a Fig. 4.3 onde se apresenta um conjunto placa-pilar nas posições inicial e deformada da placa. 


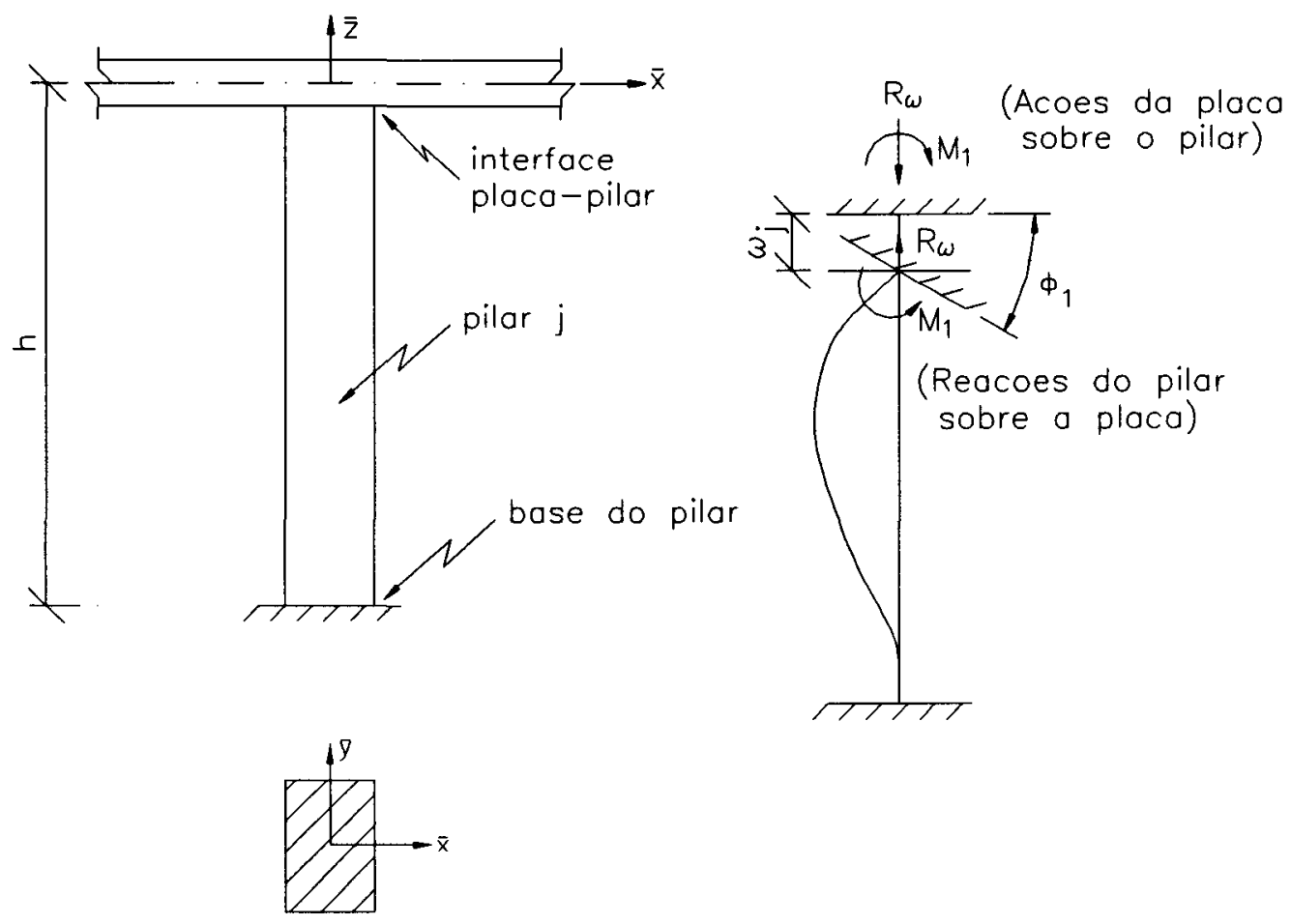

Fig. 4.3 - Conjunto placa-pilar.

Os deslocamentos da interface " $\phi_{1}, \phi_{2}$ e w" são os mesmos para a placa e o pilar. Supondo-se que estes deslocamentos fossem prescritos, as incógnitas seriam " $\mathbf{M}_{1}, \mathbf{M}_{2} \in \mathbf{R}_{\mathrm{w}}$ ", podendo o problema ser resolvido conforme a equação (4.7), onde os termos da matriz " $S$ " seriam obtidos a partir de (4.13) para reações unitárias, levados na equação (3.44), separando-se os termos constantes e os dependentes de " $r$ ".

Geralmente nem os deslocamentos nem as reaçōes da interface placa-pilar são conhecidos. Neste caso torna-se necessário relacionar tais deslocamentos com seus esforços correspondentes. Como a estrutura formada pelos pilares é um conjunto de barras independentes, para se obter a relação deslocamento-reação para cada interface placa-pilar, basta considerar apenas o pilar em questão, obtendo-se: 


$$
\left\{\begin{array}{l}
M_{1} \\
M_{2} \\
R_{w}
\end{array}\right\}_{j}=-\left[\begin{array}{lll}
k_{11} & & \underline{0} \\
& k_{22} & \\
0 & & k_{33}
\end{array}\right]_{j}\left\{\begin{array}{l}
\phi_{1} \\
\phi_{2} \\
w
\end{array}\right\}_{j}
$$

com

$$
\begin{aligned}
& k_{11}=k_{p} \frac{E I_{\overline{y y}}}{h} \\
& k_{22}=k_{p} \frac{E I_{\overline{x x}}}{h} \\
& k_{33}=\frac{E S}{h}
\end{aligned}
$$

onde:

E - módulo de elasticidade longitudinal do material do pilar.

$\mathrm{I} \overline{\mathrm{Xx}}$, Iyy - momentos de inércia em relação aos eixos “ $\bar{x}$ " $\mathrm{e}$ " $\bar{y}$ ".

S - área da seção transversal do pilar (área da interface).

h - altura do pilar.

$\mathrm{K}_{\mathrm{p}} \quad$ - um coeficiente que depende das condições de apoio na base do pilar: $\quad k_{p}=3$ - pilar articulado na base

$k_{p}=4$ - pilar engastado na base

O sinal negativo que aparece em (4.14) deve-se ao fato de que para deslocamentos positivos as reações são negativas. A convenção dos valores positivos para as reações e deslocamentos está apresentada na Fig. 4.2.a.

Levando-se (4.14) em (4.13) obtém-se: 


$$
\begin{aligned}
& A_{1}=-\frac{k_{p} E}{h} \phi_{1} \\
& B_{1}=-\frac{k_{p} E}{h} \phi_{2} \\
& \sigma(\xi)=-\frac{E}{h} w+A_{1}\left[x_{0}-x_{1}(\xi)+B_{1}\left[y_{0}-x_{2}(\xi)\right]\right]
\end{aligned}
$$

A equação (4.12) que representa a distribuição linear de tensões na área de interface, será escrita a partir de (4.16) em função dos deslocamentos " $\phi_{1}, \phi_{2}$ e w", resultando :

$$
\begin{aligned}
& \sigma(x)=\frac{k_{p} E}{h}\left[x_{1}(\xi)-x_{0}+r \cos \theta\right] \phi_{1}+ \\
& \frac{k_{p} E}{h}\left[x_{2}(\xi)-y_{0}+r \operatorname{sen} \theta\right] \phi_{2}-\frac{E}{h} w
\end{aligned}
$$

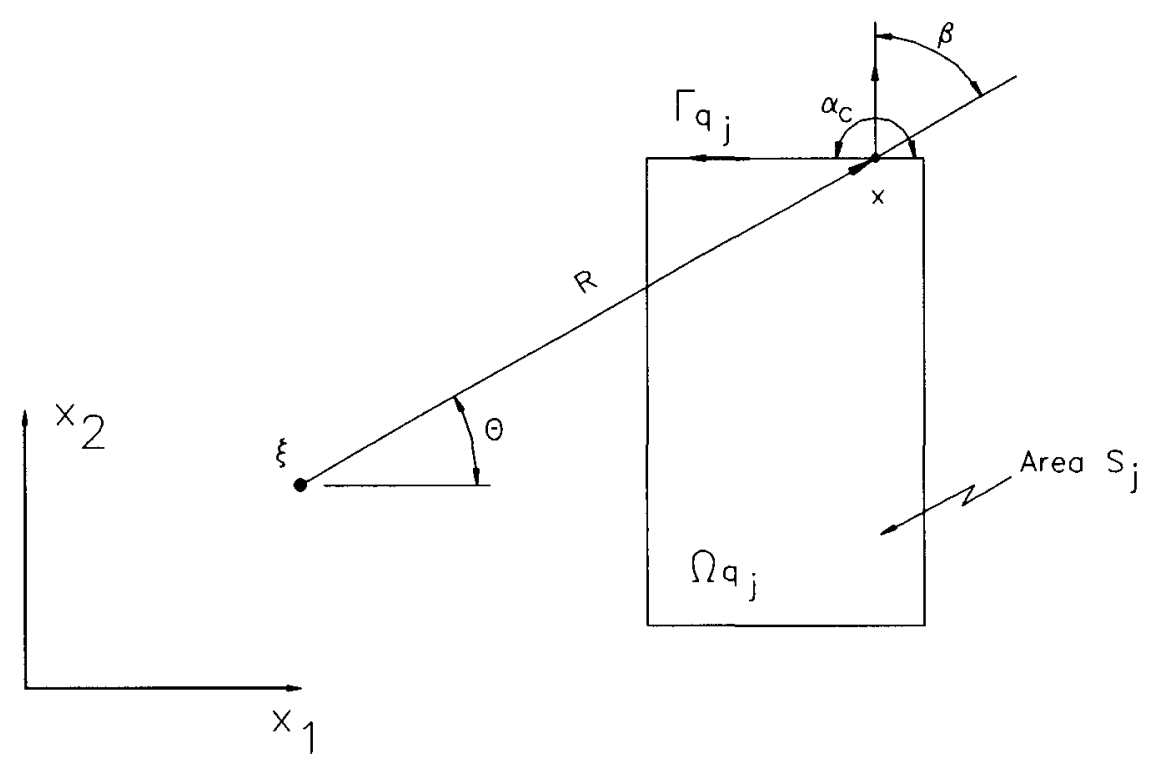

Fig. 4.4 - Área carregada " $S_{j}$. 
A influência da carga " $\sigma(x)$ " atuando na área " $S_{j}$ ", no cálculo dos deslocamentos é dada segundo (3.38) por:

$$
I_{i}=\int_{\Omega_{q s}} \sigma(x)\left[u_{i 3}^{*}(\xi, x)-\frac{v}{(1-v) \lambda^{2}} u_{i \alpha, \alpha}^{*}(\xi, x)\right] d \Omega_{q_{s}}(x)
$$

Este integral no domínio interno " $\Omega_{q}$ " será transformada em uma integral sobre o contorno " $\Gamma_{q_{s}}$ ", situação ilustrada na Fig. 4.4, conforme (3.44), resultando:

$$
\begin{aligned}
& I_{\alpha}=\frac{k_{p} E}{h}\left\{\left[x_{1}(\xi)-x_{0}\right] I_{\alpha_{c}}+I_{a_{r 1}}\right\} \phi_{1}+\frac{k_{p} E}{h}\left\{\left[x_{2}(\xi)-y_{0}\right] I_{\alpha_{c}}+I_{a_{r 2}}\right\} \phi_{2}-\frac{E}{h} I_{\alpha_{c}} w(4.19) \\
& I_{3}=\frac{k_{p} E}{h}\left\{\left[x_{1}(\xi)-x_{0}\right] I_{3_{c}}+I_{3_{r 1}}\right\} \phi_{1}+\frac{k_{p} E}{h}\left\{\left[x_{2}(\xi)-y_{0}\right] I_{3_{c}}+I_{3_{r 2}}\right\} \phi_{2}-\frac{E}{h} I_{3_{c}} w
\end{aligned}
$$

onde:

$$
\begin{aligned}
& I_{\alpha_{c}}=\frac{1}{24 \pi D} \int_{\Gamma_{q_{s}}}\left[R^{2}\left(2 \ln z-\frac{5}{3}\right)+\frac{12 v}{(1-v) \lambda^{2}}\right] r_{, \alpha} \cos \beta d \Gamma_{q_{s}}(x) \\
& I_{\alpha_{r \beta}}=\frac{1}{32 \pi D} \int_{\Gamma_{q_{s}}}\left[R^{3}\left(2 \ln z-\frac{3}{2}\right)+\frac{8 v}{(1-v) \lambda^{2}} R\right]_{, \alpha} r_{, \beta} \cos \beta d \Gamma_{q_{s}}(x) \\
& I_{3_{c}}=\frac{1}{8 \pi D(1-v) \lambda^{2}} \int_{\Gamma_{q_{s}}} R\left[\frac{(1-v)}{4} z^{2}\left(\ln z-\frac{5}{4}\right)+2(v-2)\left(\ln z-\frac{1}{2}\right)\right] \cos \beta d \Gamma_{q_{s}}(x) \\
& I_{3_{r a}}=\frac{1}{8 \pi D(1-v) \lambda^{2}} \int_{\Gamma_{q_{s}}} R^{2}\left[\frac{(1-v)}{5} z^{2}\left(\ln z-\frac{6}{5}\right)+\frac{4}{3}(v-2)\left(\ln z-\frac{1}{3}\right)\right],_{, \alpha} \cos \beta d \Gamma_{q_{s}}(x)
\end{aligned}
$$

com: 


$$
\begin{gathered}
r_{, \alpha}=\left\{\begin{array}{l}
\cos \theta \\
\operatorname{sen} \theta
\end{array}\right. \\
\cos \beta=r_{, n}=\cos \theta \operatorname{sen} \alpha_{c}-\operatorname{sen} \theta \cos \alpha_{c}
\end{gathered}
$$

" $\theta$ " e " $\alpha_{c}$ " estão ilustradas na Fig. 4.4.

Considerando-se uma placa com "NAP" apoios internos, conforme o apoio genérico "j" da Fig. 4.2, pode-se escrever:

$$
\underset{\sim}{H} \underset{\sim}{U}=\underset{\sim}{G} \underset{\sim}{P}+\underset{\sim}{B}+\underset{\sim}{Q} U_{\sim}
$$

onde a matriz " $Q$ " de dimensão "3(NNCP) x 3(NAP)" contém os coeficientes expressos em (4.19) e (4.20) e o vetor " $U_{s}$ " contém os deslocamentos " $\phi_{1}$, $\phi_{2} \odot w^{\prime \prime}$ para todos os "NAP" apoios internos.

Como os deslocamentos na interface são os mesmos para a placa e para o pilar, pode-se escrever:

$$
U_{s}=U_{i}
$$

Para que o sistema representado pela equação (4.23) possa ser resolvido, torna-se necessário a criação de "3(NAP)" novas equações, que serão as correspondentes aos " $U_{i}$ " deslocamentos internos. Assim tem-se:

$$
U_{i}=G_{i} \underset{\sim}{P}-H_{i} \underset{\sim}{U}+B_{i}+Q_{\sim} U_{i}
$$


onde " $\underline{\sim}_{i}$ " representa a matriz " $\underset{\sim}{ }$ " para pontos internos. Deve-se notar que " $Q_{i}{ }^{n}$ não apresenta singularidades, porque os deslocamentos nos pontos internos são calculados para o centro de gravidade da área de interface, de coordenadas globais " $x_{0}, y_{0}$ ", e a integraçăo no domínio " $\Omega_{q_{s}}$ " é feita sobre 0 contorno " $\Gamma_{q_{s}}$.

Agrupando-se (4.23) e (4.25) obtém-se:

$$
\left[\begin{array}{cc}
\underset{H}{H} & 0 \\
\tilde{H}_{i} & \tilde{I} \\
\sim & \sim
\end{array}\right]\left\{\begin{array}{c}
\underset{\sim}{\tilde{U}_{i}} \\
\sim
\end{array}\right\}=\left[\begin{array}{c}
G \\
\tilde{G}_{i} \\
\sim
\end{array}\right] \underset{\sim}{P+}\left[\begin{array}{c}
B \\
\tilde{B}_{i} \\
\sim
\end{array}\right]+\left[\begin{array}{c}
\underset{Q}{\tilde{Q}_{i}} \\
\underset{\sim}{\tilde{Q}_{i}}
\end{array}\right] \underset{\sim}{U_{i}}
$$

Levando-se os deslocamentos incógnitos " $U_{i}$ " do segundo para o primeiro membro, obtém-se:

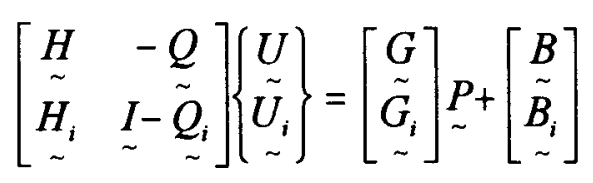

Impondo-se as condições de contorno, o sistema (4.27) é resolvido encontrando-se além dos valores de contorno, os deslocamentos nos pontos internos correspondentes aos "NAP" apoios. Com os valores " $U_{i}$ ", a partir da expressão (4.14), encontram-se as reações nos pilares. Com estas últimas obtém-se as distribuições lineares de tensão sobre todas as áreas de interface, através da equaçăo (4.12). Deve-se notar que estas cargas lineares sobre as áreas de interface poderiam ser também obtidas através de (4.17), sem a necessidade de se ter calculado as reações dos pilares. 
Os deslocamentos em outros pontos internos serão obtidos a partir da equação (3.95), onde o vetor " $B_{i}$ " conterá a influência de todos os carregamentos de domínio, inclusive das "NAP" regiōes carregadas com as cargas " $\sigma(x)$ ", dada em (4.12).

Para o cálculo dos esforços em pontos internos, inclusive nos centros

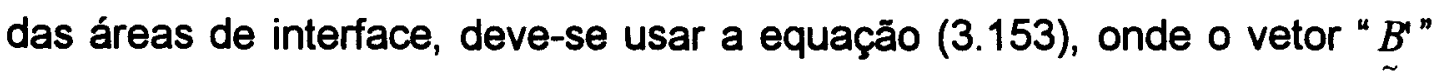
conterá a influência de todo o carregamento interno, inclusive destas novas regiōes carregadas com " $\sigma(x)$ ".

Mesmo quando se desprezam as reaçōes " $M_{1}{ }^{n}$ e " $M_{2}{ }^{n}$, tomando-se " $\sqrt{x x}=\sqrt{y y}=0 "$, é conveniente usar esta formulação, por menor que seja a área da interface placa-pilar. A tensão " $\sigma(x)$ " neste caso é constante e igual a tensão média $\left(\sigma(x)=R_{w} / s\right)$. Para rigidez axial infinita a incógnita é a tensão " $\sigma(x)$ ", quando considera-se a rigidez axial real do pilar, a incógnita será 0 deslocamento " $w$ ". A reação distribuída uniformemente na área de interface, conduz a resultados melhores, pois se evitam desta forma as singularidades que apareceriam no ponto de aplicação da reação, caso a mesma fosse considerada concentrada.

\section{3 - PLACAS COM ENRIJECEDORES}

\subsection{1 - ASSOCIAÇÃO PLACA-ESTRUTURA QUALQUER}

Pretende-se neste item analisar a associação de uma placa com uma estrutura qualquer, formada por barras. Para isto considera-se que os novos elementos estruturais estejam ligados à placa por linhas e pontos discretos. 
Estas linhas são na realidade vigas associadas à placa, com rigidezes à flexão e torção, impondo-se assim um certo enrijecimento à mesma.

Os esforços que surgirão na interface das duas estruturas podem ser interpretados como linhas de carga vertical e linhas de momentos aplicados à placa.

Seja a Fig. 4.5, onde se apresenta uma placa com uma linha genérica, mostrando-se as linhas de carga e de momentos.

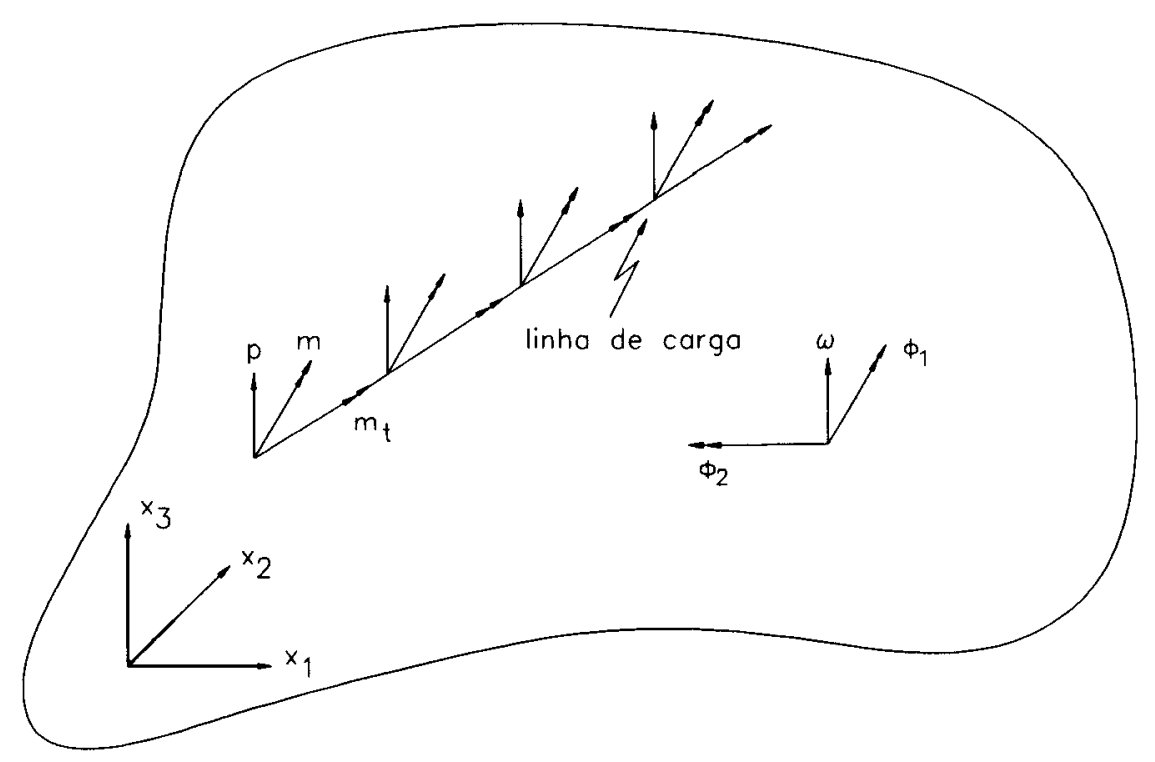

Fig. 4.5 - Placa com uma linha genérica de cargas da interface.

As linhas da interface serão discretizadas em elementos conforme item 3.3.3, onde os valores das reações serão aproximados segundo (3.90) para cargas distribuídas verticais ou segundo (3.92) para linhas de momentos distribuídos. Desta forma a equação para deslocamentos no contorno, representada matricialmente por (3.86), será escrita como:

$$
\underset{\sim}{H} \underset{\sim}{U}=\underset{\sim}{G} \underset{\sim}{P}+\underset{\sim}{B}+\underset{\sim}{S} p^{N}
$$

onde: 
$p^{N} \quad$ - é o vetor dos valores nodais das linhas de carga vertical e de momentos, interpretadas como reações da estrutura sobre a placa.

$\stackrel{\sim}{S} \quad$ - é uma matriz cujos coeficientes representam a influência das linhas de carga.

O vetor " $p$ " para um ponto qualquer da linha de carga é dado por:

$$
\underset{\sim}{p}=\left\{\begin{array}{l}
m_{t} \\
m \\
p
\end{array}\right\}
$$

onde " $m_{t}$ " é o momento de torção, " $m$ " é o momento fletor e " $p$ " é carga vertical, todos considerados por unidade de comprimento. $O$ vetor dos valores nodais destas linhas de carga para um elemento genérico de carga uj", conforme aproximação quadrática adotada no item 3.3.3, é dado por:

$$
\underset{\sim j}{p^{N^{r}}}=\left\{\begin{array}{lllllllll}
m_{t}^{1} & m^{1} & p^{1} & m_{t}^{2} & m^{2} & p^{2} & m_{t}^{3} & m^{3} & p^{3}
\end{array}\right\}_{j}
$$

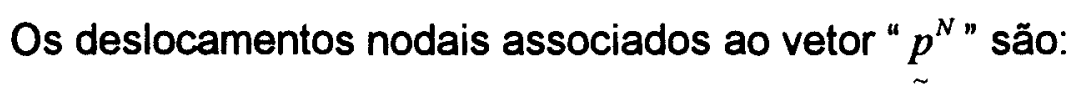

$$
\underset{\sim_{j}}{\boldsymbol{u}^{N^{r}}}=\left\{\begin{array}{lllllllll}
\gamma^{1} & \theta^{1} & w^{1} & \gamma^{2} & \theta^{2} & w^{2} & \gamma^{3} & \theta^{3} & w^{3}
\end{array}\right\}_{j}
$$

O sistema local de referência para um elemento da linha de carga está ilustrado na Fig. 4.6. A direçăo "1" coincide com o eixo da barra e está associada à linha de momentos de torção " $\mathrm{m}_{\mathrm{t}}$ " e à correspondente rotação devido à torção " $\gamma$ ". A direção " 2 " é perpendicular ao eixo da barra e corresponde à linha de momentos fletores " $m$ " e à rotação " $\theta$ " respectiva. A 
direção " 3 " coincide com a direção " $x_{3}$ " global, e está associada à linha de carga vertical e ao correspondente deslocamento transversal " $W$ ".

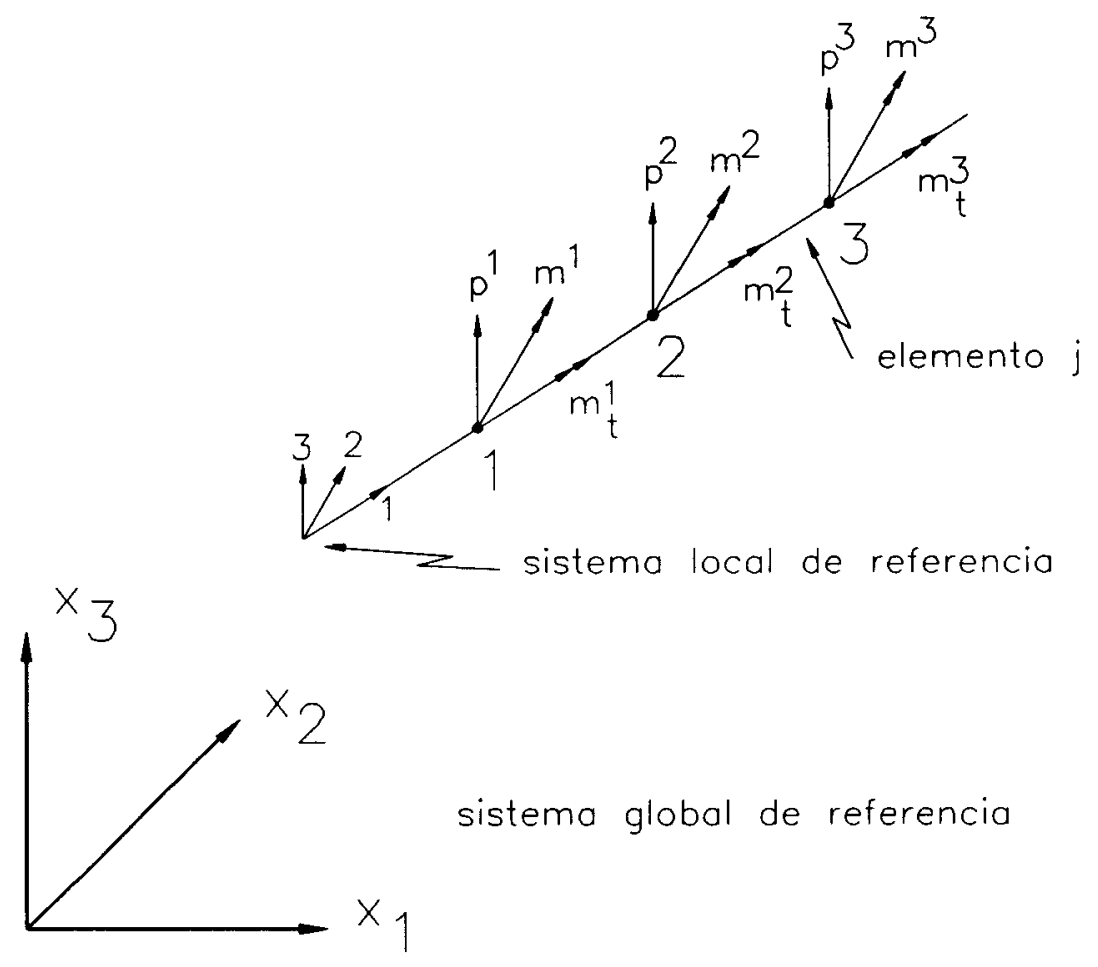

Fig. 4.6 - Elemento genérico da ligaçăo placa-estrutura.

A matriz " $\underset{\sim}{S}$ em (4.28) é obtida a partir da influência das linhas de carga vertical e de momentos no cálculo dos deslocamentos, expressas respectivamente pelas equaçōes (3.50) e (3.65). Considerando-se (3.90) e (3.92) o último termo de (4.28) pode ser escrito para um elemento genérico uj": 


$$
\underset{\sim}{S_{j} p_{j}}=\left[\begin{array}{ccc}
S_{j} \phi_{1} & S_{j} \phi_{2} & S_{j} \phi_{3}
\end{array}\right]\left\{\begin{array}{c}
m_{i}^{1} \\
m^{1} \\
p^{1} \\
m_{t}^{2} \\
m^{2} \\
p^{2} \\
m_{t}^{3} \\
m^{3} \\
p^{3}
\end{array}\right\}_{j}^{S_{j} p_{j}=\underset{\sim}{S_{\sim}^{*} p_{\sim j}^{N}}}
$$

onde as funções " $\phi_{i}$ " são dadas em (3.79).

A matriz " $S_{j}$ " é uma matriz quadrada de ordem 3 , sendo seus coeficientes obtidos pelas seguintes integraçŏes sobre o elemento "j":

$$
\begin{aligned}
& S_{\alpha \beta}=\int_{\Gamma_{j}} u_{\alpha \beta}^{*}(\chi, x) d \Gamma_{j}(x) \\
& S_{3 \alpha}=\int_{\Gamma_{j}} u_{3 \alpha}^{*}(\chi, x) d \Gamma_{j}(x) \\
& S_{i 3}=\int_{\Gamma_{j}}\left[u_{i 3}^{*}(\chi, x)-\frac{v}{(1-v) \lambda^{2}} u_{i \alpha, \alpha}^{*}(\chi, x)\right] d \Gamma_{j}(x)
\end{aligned}
$$

onde as soluçōes fundamentais " $u_{i j}^{*}$ " estão dadas em (2.32) e " $u_{i \alpha, \alpha}^{*}$ " em (3.21).

Mesmo quando a linha de carga coincide com o contorno da placa, as integrais (4.33) não apresentam singularidades, pois o ponto " $\chi$ " do contorno 
foi levado para fora do domínio, conforme mostrado no item 3.3.2. Estas integrais serão todas resolvidas numericamente.

Analisando-se a equação (4.28), nota-se que além das incógnitas de contorno, deslocamentos ou forças de superfície, aparecem também as cargas nodais distribuidas, representadas no vetor " $p^{N n}$. As cargas nodais distribuídas não são valores incógnitos normais, na análise de placas, como o são deslocamentos e forças. Além do mais năo se garante a continuidade

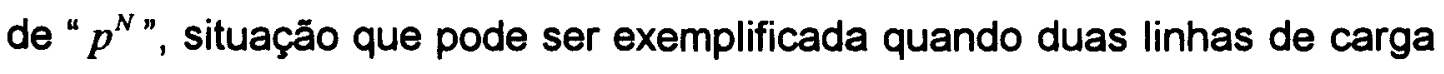
se cruzam. No ponto de cruzamento pode-se ter 4 valores distintos para 0 vetor " $p$ ". Isto não acontece com os deslocamentos, que são os mesmos para o ponto de cruzamento, tanto na placa quanto na estrutura associada.

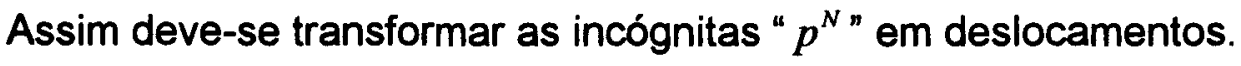

A transformação do vetor " $p^{N "}$ em deslocamentos se fará considerando-se isoladamente cada elemento genérico " $\mathrm{j}$ " da linha de carga. Os esforços nodais do elemento "j" se relacionam com os deslocamentos nodais correspondentes, através de:

$$
\underset{\sim}{k} \underset{e_{j}, e_{j}}{u}=F_{j}
$$

onde:

$\underset{\sim}{k} \quad$ - é a matriz de rigidez do elemento ${ }^{4 j}$.

${\underset{\sim}{e_{j}}}_{u}^{u} \quad$ é o vetor de deslocamentos nodais do elemento "j".

$F_{j}$ - é o vetor das forças nodais equivalentes do elemento "j", correspondentes à aplicação das linhas de carga vertical e de momentos. 
$\mathrm{Na}$ análise aqui desenvolvida o enrijecimento produzido pelas vigas é traduzido pelas linhas de carga " $p$ ", que é equivalente à aplicação do vetor " $F_{j}$ " no elemento. Por outro lado, sabe-se que a aplicação de " $F_{j}$ " produz no elemento deslocamentos “ ${ }_{\sim e j}$ ", que são proporcionais à rigidez da barra "k $\underset{\sim}{k}$ ". Desta forma consegue-se relacionar as linhas de carga " $\underset{\sim}{p}$ com os deslocamentos " $u_{e}$ ", que são os mesmos para a viga e para a placa na interface.

Consegue-se transformar o vetor dos valores nodais das linhas de carga " $p$ " no vetor das forças nodais equivalentes através da seguinte equação:

$$
F_{\sim}=\underset{\sim}{N} \underset{\sim}{p^{N}}
$$

onde " $N$ " é dado a partir do Método dos Elementos Finitos por:

$$
\underset{\sim}{N}=\frac{l}{2} \int_{-1}^{+1} \underset{\sim v}{\phi_{\nu}^{T}}(\eta) \underset{\sim}{\phi_{p}}(\eta) d \eta
$$

A matriz " $N$ " transforma cargas nodais distribuidas em forças nodais equivalentes. Os vetores " $\phi_{v}(\eta)$ " e " $\phi_{p}(\eta)$ são funçăo da aproximação adotada para os deslocamentos " $v(\eta)$ " e para as cargas distribuídas " $p(\eta)$ ". Assim:

$$
\begin{aligned}
& \underset{\sim}{v}(\eta)=\underset{\sim}{\phi_{v}}(\eta) u_{e_{j}}^{N} \\
& \underset{\sim}{p}(\eta)=\underset{\sim}{\phi_{p}}(\eta){\underset{\sim}{e_{j}}}^{N}
\end{aligned}
$$


As aproximaçōes adotadas para " $\underset{\sim}{v}(\eta)^{n}$ e " $\underset{\sim}{p(\eta) " ~ s e r a ̃ o ~ a n a l i s a d a s ~}$ posteriormente no item 4.3.2.

Substituindo-se (4.35) em (4.34) obtém-se:

$$
\underset{\sim}{k} \underset{\sim}{u}=\underset{\sim}{N} \underset{\sim}{p^{N}}
$$

Multiplicando-se os dois membros de (4.38) por $\mathrm{N}^{-1}$, obtém-se a transformação do vetor " $p^{N}$ " no vetor dos deslocamentos " $u_{e}$ ".

$$
N_{\sim}^{-1} \underset{\sim}{\boldsymbol{k}} \underset{\sim e}{u}=\boldsymbol{p}^{N}
$$

De (4.32) pode-se escrever:

$$
S_{\sim} p_{j}^{N}=S_{\sim} N_{j}^{-1} k_{e j} u_{e j}
$$

Resolve-se (4.40) para cada elemento das linhas de carga, endereçam-se e armazenam-se os coeficientes de todos os deslocamentos " $u_{e}$ " formando-se uma matriz " $\underset{\sim N K}{S}$ " com "3(NNCP)" linhas e "3(NNLC)" colunas, onde "NNLC" é o número de nós que definem linhas de cargas. Desta forma pode-se escrever (4.28):

$$
\underset{\sim}{H} \underset{\sim}{U}=\underset{\sim}{G} \underset{\sim}{P}+\underset{\sim}{B}+\underset{\sim N K}{S} \underline{\sim}_{\sim}
$$

O vetor dos deslocamentos " $u_{e}$ " pode conter tanto deslocamentos internos como do contorno, ficando (4.41) expresso da seguinte forma: 


$$
\underset{\sim}{H} \underset{\sim}{U}=\underset{\sim}{G} \underset{\sim}{P}+\underset{\sim}{B}+\left[\underset{\sim}{S} \quad{\underset{\sim}{S}}_{N K_{\mathrm{rn}}}^{S}\right]\left\{\begin{array}{c}
U \\
\tilde{U}_{i} \\
\sim
\end{array}\right\}
$$

$\mathrm{Na}$ expressão (4.42) foram introduzidas novas incógnitas " $U_{i}$ ", necessitando-se portanto escreverem-se novas equações de deslocamentos para os pontos internos envolvidos no vetor " $U_{i}$ ". Agrupando-se estas novas equações à equação (4.42), obtém-se:

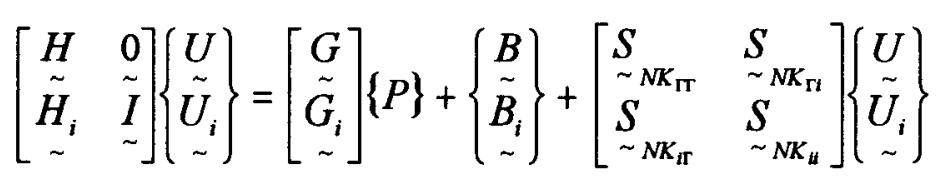

Levando-se os deslocamentos " $U^{\prime}$ " e $U_{i}$ " para o primeiro membro de (4.43) resulta:

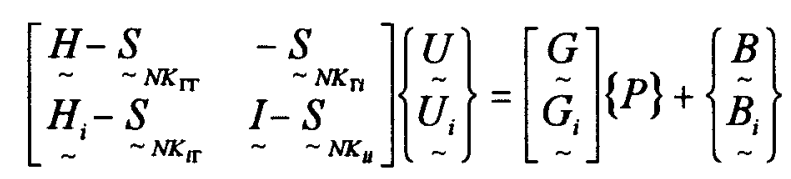

A equação (4.44) representa a equação matricial geral (3.86), transformada agora para levar em conta o enrijecimento produzido pelas vigas, que é traduzido pela aplicação das linhas de carga.

Impondo-se as condições de contorno, resolve-se o sistema (4.44), determinando-se as incógnitas de contorno e os deslocamentos dos pontos internos, pertencentes a interface da estrutura formada pelas vigas com a placa. Com os deslocamentos conhecidos determinam-se para cada elemento de carga o valor de " $p^{N}$, através da equação (4.39). A partir dos 
valores de " $p^{N n}$, todo o enrijecimento produzido pela estrutura das vigas, será introduzido na placa através da aplicação destas linhas de cargas. Assim calculam-se deslocamentos em outros pontos internos de interesse, bem como esforços em quaisquer pontos internos, aplicando-se equaçōes integrais já desenvolvidas anteriormente, que conterão agora a influência do enrijecimento produzido pelas linhas de cargas " $\boldsymbol{p}^{N}$.

A equaçăo (4.44) serve também para levar em conta o enrijecimento produzido pelos pilares, bastando para isso, acrescentar aos coeficientes correspondentes aos deslocamentos da interface placa-viga-pilar, que são os mesmos para os três elementos, as rigidezes correspondentes do pilar, já deduzidas anteriormente.

\subsection{2 - MATRIZ DE RIGIDEZ DAS BARRAS}

Conforme visto no item anterior, é necessário determinar a matriz de rigidez " $k_{\varepsilon}$ " do elemento de carga, assim como a matriz " $\underset{\sim}{N}$, que relaciona cargas distribuídas com forças nodais equivalentes. Estas duas matrizes serão definidas neste item a partir de funções aproximadoras previamente escolhidas para deslocamentos e cargas distribuidas.

O sentido dos deslocamentos para pontos da placa está indicado na Fig. 2.1.b. Como os deslocamentos da placa são os mesmos da estrutura associada, deve-se adotar um sistema global de coordenadas, para o cálculo da matriz de rigidez das barras, coerente com o sentido dos deslocamentos " $\phi_{1}$ ", " $\phi_{2}$ " e " $w$ ". O sistema local para as barras será o mesmo já adotado no item anterior na Fig. 4.6. Os dois sistemas de coordenadas estão ilustrados na Fig.4.7. 


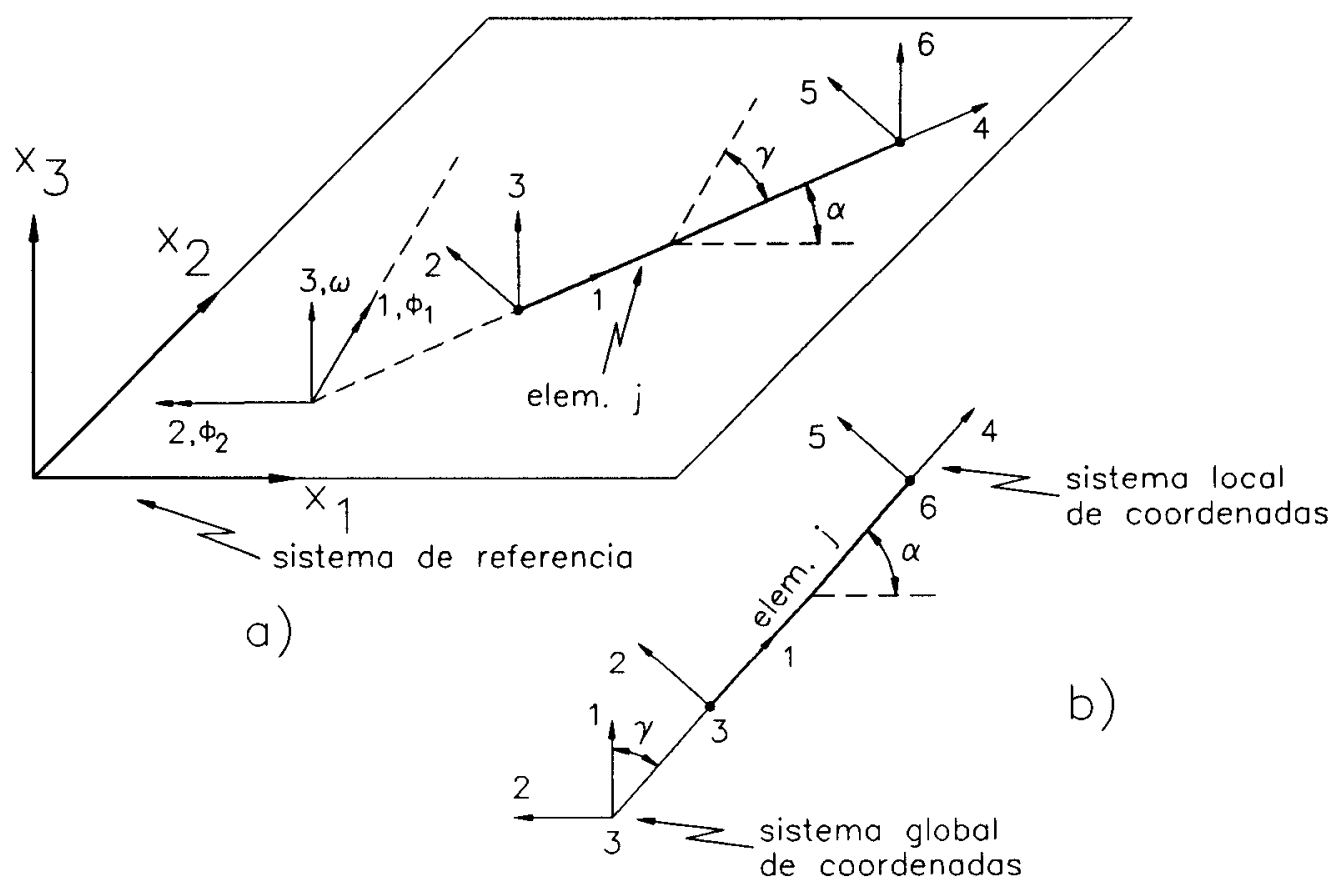

Fig. 4.7 - Sistemas global e local de coordenadas.

Seja o elemento de barra genérico "j" da Fig. 4.7.b. Os deslocamentos verticais dos pontos da barra estão relacionados com as respectivas rotações através de:

$$
\theta=-\frac{d v}{d x}
$$

onde o sinal negativo aparece porque para " $v$ " crescente a rotação " $\theta$ " é decrescente. 


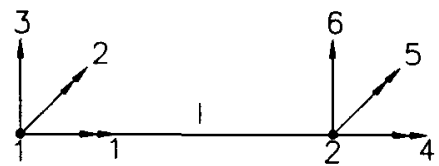

о)

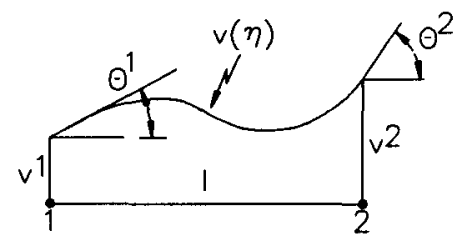

b)

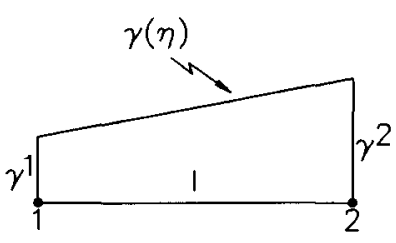

c)

Fig. 4.8 - a) Sistema local de coordenadas

b) Aproximação cúbica para $v(\eta)$

c) Aproximação linear para $\gamma(\eta)$

Os deslocamentos " $\mathrm{v}$ " e " $\theta$ " são completamente independentes da rotação por torção " $\gamma$ ". Considerando-se o deslocamento vertical e a rotação para cada um dos dois nós extremos do elemento de barra, pode-se exprimir " $v(\eta)$ " da seguinte forma:

$$
v(\eta)=\underset{\sim}{\psi}(\eta) \underset{\sim}{\alpha}=\left\{\begin{array}{llll}
1 & \eta & \eta^{2} & \eta^{3}
\end{array}\right\}\left\{\begin{array}{c}
\alpha_{0} \\
\alpha_{1} \\
\alpha_{2} \\
\alpha_{3}
\end{array}\right\}
$$

onde " $\alpha$ " é o vetor dos parâmetros generalizados e " $\eta$ " é dado por:

$$
\eta=\frac{2}{l} x
$$

Levando-se (4.47) em (4.45) obtém-se:

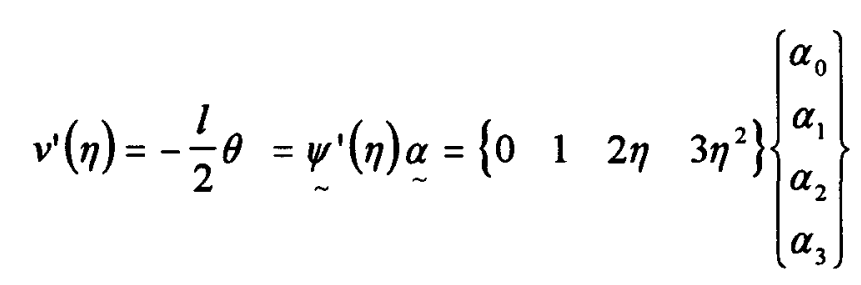


Os valores nodais dos deslocamentos " $\theta^{1 n}, " v^{1 n}$, e " $\theta^{2 n}, " v^{2 n}$ são obtidos para valores de " $\eta=-1$ " e " $\eta=+1$ " respectivamente nas expressőes (4.48) e (4.46), obtendo-se os mesmos em função dos parâmetros generalizados " $\alpha$ ". Levando-se os valores dos parâmetros generalizados " $\alpha$ " escritos em função dos valores nodais nas expressōes (4.46) e (4.48) resulta:

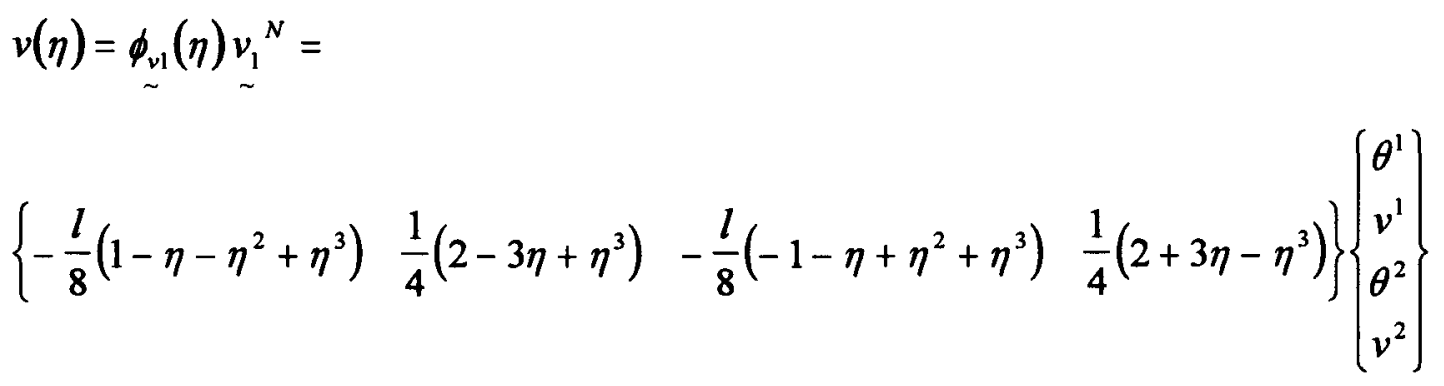

$$
\theta(\eta)=-\frac{2}{l} \nu^{\prime}(\eta)=-\frac{2}{l} \phi_{\sim}{ }_{\sim}^{\prime}(\eta) \underset{\sim}{v_{1}^{N}}=
$$$$
=\left\{\frac{1}{4}\left(-1-2 \eta+3 \eta^{2}\right)-\frac{1}{2 l}\left(-3+3 \eta^{2}\right) \frac{1}{4}\left(-1+2 \eta+3 \eta^{2}\right)-\frac{1}{2 l}\left(3-3 \eta^{2}\right)\right\}\left\{\begin{array}{c}
\theta^{1} \\
v^{1} \\
\theta^{2} \\
v^{2}
\end{array}\right\}
$$

A partir da variaçăo linear para a rotação devido a torção " $\gamma$ ", conforme Fig. 4.8.c, e seguindo-se procedimento análogo ao utilizado para a flecha " $v$ " e a rotação " $\theta$ ", chega-se a:

$$
\gamma(\eta)=\phi_{\sim}(\eta) \underset{\sim}{v_{2}^{N}}=\left\{\begin{array}{ll}
\frac{1}{2}(1-\eta) & \frac{1}{2}(1+\eta)
\end{array}\right\}\left\{\begin{array}{l}
\gamma_{1} \\
\gamma_{2}
\end{array}\right\}
$$

Agrupando-se (4.49) a (4.51) obtém-se: 
$\underset{\sim}{u(\eta)}=\left\{\begin{array}{c}\gamma(\eta) \\ \theta(\eta) \\ v(\eta)\end{array}\right\}=\underset{\sim}{\phi} \underset{\nu}{ }(\eta){\underset{\sim}{v}}^{N}=$

(4.52)

$=\left[\begin{array}{cccccc}\frac{1}{2}(1-\eta) & 0 & 0 & \frac{1}{2}(1+\eta) & 0 & 0 \\ 0 & \frac{1}{4}\left(-1-2 \eta+3 \eta^{2}\right) & \frac{-1}{2 l}\left(-3+3 \eta^{2}\right) & 0 & \frac{1}{4}\left(-1+2 \eta+3 \eta^{2}\right) & \frac{1}{2 l}\left(3-3 \eta^{2}\right) \\ 0 & -\frac{l}{8}\left(1-\eta-\eta^{2}+\eta^{3}\right) & \frac{1}{4}\left(2-3 \eta+\eta^{3}\right) & 0 & \frac{-l}{8}\left(-1-\eta+\eta^{2}+\eta^{3}\right) & \frac{1}{4}\left(2+3 \eta-\eta^{3}\right)\end{array}\right]\left\{\begin{array}{c}\gamma^{1} \\ \theta^{1} \\ v^{1} \\ \gamma^{2} \\ \theta^{2} \\ v^{2}\end{array}\right\}$ 
A energia de deformação para a barra genérica " $\mathrm{j}$ ", considerando-se apenas a torção e a flexão é dada por:

$$
U=\frac{1}{2} \int_{-l / 2}^{+l / 2} \frac{M_{t}^{2}}{G J_{t}} d x+\frac{1}{2} \int_{-l / 2}^{+/ / 2} \frac{M^{2}}{E I} d x
$$

onde:

$$
\begin{aligned}
& M_{t}=G J_{t} \gamma^{\prime}(x)=G J_{t} \frac{2}{l} \gamma^{\prime}(\eta) \\
& M=E I v^{\prime \prime}(x)=E I \frac{4}{l^{2}} v^{\prime \prime}(\eta)
\end{aligned}
$$

com:

E - módulo de elasticidade longitudinal

G - módulo de elasticidade transversal

I - momento de inércia à flexão da seção da barra em relação ao eixo local 2

$\mathrm{J}_{\mathrm{t}} \quad$ - momento de inércia à torção da seção da barra em relação ao eixo 1

$\ell \quad$ - é o comprimento do elemento de barra

Considerando-se seção transversal retangular "bxh", com " $h$ " paralelo à direção 3 , tem-se "Jit" calculado por:

$$
J_{t}=\beta h b^{3}
$$

onde:

$$
\beta=\frac{1}{3}-0,21 \frac{b}{h}\left(1-\frac{b^{4}}{12 h^{4}}\right)
$$


Substituindo-se (4.54) e (4.55) em (4.53) e mudando-se a variável " $x$ " para " $\eta$ ", obtém-se:

$$
\begin{aligned}
& U=\frac{1}{2}{\underset{\sim}{2}}^{N^{T}}\left(\frac{2}{l} \int_{-1}^{+1}{\underset{\sim}{\phi_{2}}}^{{ }^{T}}(\eta) G J_{t}{\underset{\sim}{\phi}}_{2}(\eta) d \eta\right) \underset{\sim}{v_{2}{ }^{N}+} \\
& \frac{1}{2} \underset{\sim}{v_{1}{ }^{T}}\left(\frac{8}{l^{3}} \int_{-1}^{+1}{\underset{\sim}{1}}^{1^{T}}(\eta) E I \underset{\sim}{\phi_{1}^{\prime}}(\eta) d \eta\right) \underset{\sim}{v_{1}^{N}}
\end{aligned}
$$

Os termos entre parênteses da equação (4.57) representam a matriz de rigidez do elemento de barra, sendo o primeiro termo devido à torção e o segundo devido à flexão. Integrando-se obtém-se uma matriz $(2 \times 2)$ para o primeiro termo, e uma matriz $(4 \times 4)$ para o segundo, obtidas independentemente. Estas matrizes agrupadas geram uma matriz $(6 \times 6)$ que representa a rigidez da barra em relação ao sistema local, dada por:

$$
\boldsymbol{k}_{e}=\left[\begin{array}{cccccc}
\frac{G J_{t}}{l} & 0 & 0 & -\frac{G J_{t}}{l} & 0 & 0 \\
& \frac{4 E I}{l} & -\frac{6 E I}{l^{2}} & 0 & \frac{2 E I}{l} & \frac{6 E I}{l^{2}} \\
& & \frac{12 E I}{l^{3}} & 0 & -\frac{6 E I}{l^{2}} & -\frac{12 E I}{l^{3}} \\
& & & \frac{G J_{t}}{l} & 0 & 0 \\
& \text { Sime'tric a } & & & \frac{4 E I}{l} & \frac{6 E I}{l^{2}} \\
& & & & & \frac{12 E I}{l^{3}}
\end{array}\right]
$$

A matriz de rigidez (4.58) escrita em relação ao sistema global de coordenadas é dada por: 


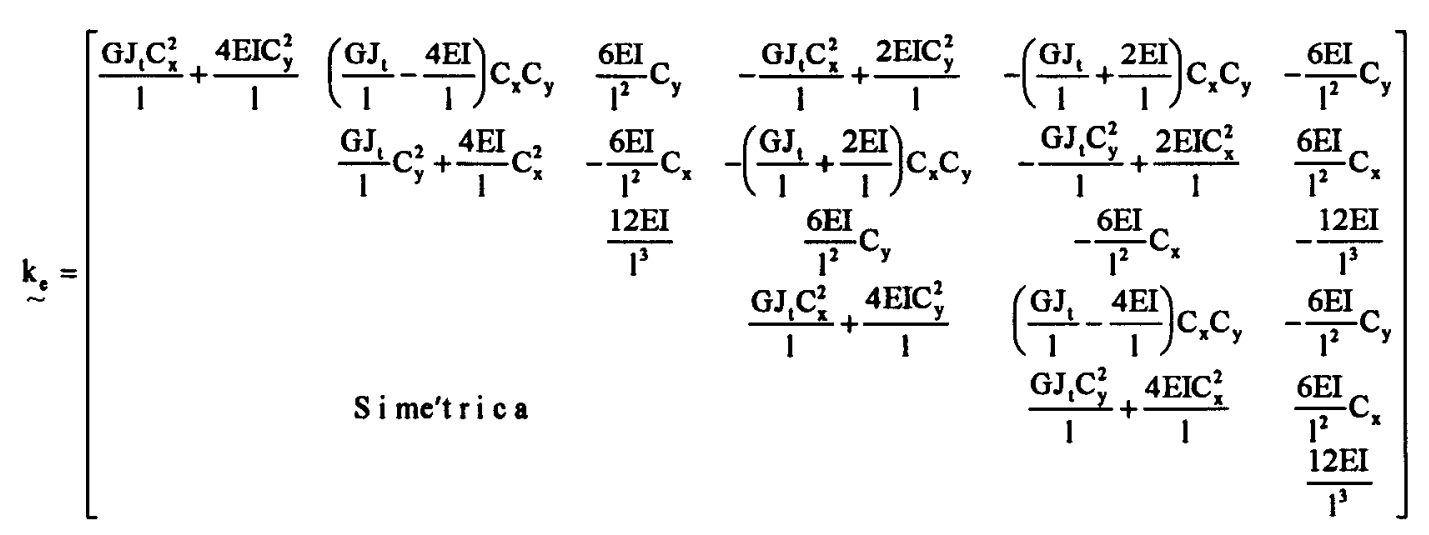

onde:

$$
\begin{aligned}
& C_{x}=\cos \gamma=\operatorname{sen} \alpha \\
& C_{y}=\operatorname{sen} \gamma=-\cos \alpha
\end{aligned}
$$

onde " $\gamma$ " é o ângulo formado entre as direções 1 , dos dois sistemas de coordenadas e " $\alpha$ " é o ângulo que a direção da barra faz com o eixo " $x_{1}$ " (ver Fig. 4.7).

Considerando-se variação linear para as cargas distribuídas, pode-se escrever:

$$
\begin{aligned}
& \underset{\sim}{p(\eta)}=\left\{\begin{array}{l}
m_{t}(\eta) \\
m(\eta) \\
p(\eta)
\end{array}\right\}=\underset{\sim}{\phi_{p}(\eta) \underset{\sim}{p^{N}}=} \\
& \left.=\left[\begin{array}{cccccc}
\frac{1}{2}(1-\eta) & 0 & 0 & \frac{1}{2}(1+\eta) & 0 & 0
\end{array}\right] \begin{array}{l}
m_{t}^{1} \\
m^{1} \\
p^{1} \\
m_{t}^{2} \\
m^{2} \\
p^{2}
\end{array}\right\}
\end{aligned}
$$


A variação da energia potencial produzida pelo carregamento " $p$ " é dada por:

$$
\Omega=-W=-\left(\int_{-l / 2}^{+1 / 2} m_{t}(x) \gamma(x) d x+\int_{-l / 2}^{+1 / 2} p(x) v(x) d x\right)
$$

Substituindo-se os valores de " $m_{4}(x)^{n}$ e " $p(x)$ " dados em (4.61), o valor de " $\gamma(x)$ " dado em (4.51), o valor de " $v(x)^{n}$ dado em (4.49) e mudando-se a variável " $x$ " para " $\eta$ ", obtém-se:

$$
\Omega=-\underset{\sim}{v_{2}} N^{T}\left(\frac{l}{2} \int_{-1}^{+1} \underset{\sim}{\phi_{v_{2}}}(\eta) \underset{\sim}{\phi_{p_{2}}}(\eta) d \eta\right)-\underset{\sim}{v_{1}}{ }^{T}\left(\frac{l}{2} \int_{-1}^{+1} \underset{\sim}{\phi_{\nu_{1}}}{ }^{T}(\eta) \underset{\sim}{\phi_{p_{1}}}(\eta) d \eta\right)(4.63)
$$

Os termos entre parênteses da equação (4.63) representam a matriz " $N$ " referida anteriormente pela expressão (4.36), sendo o primeiro termo correspondente à torção e o segundo à flexão. Integrando-se obtém-se uma matriz (2x2) para o primeiro termo e uma matriz $(4 \times 4)$ para o segundo, obtidas de forma independente. Estas matrizes agrupadas convenientemente formam uma matriz $(6 \times 6)$ dada por:

$$
\underset{\sim}{N}=\left[\begin{array}{cccccc}
\frac{l}{3} & 0 & 0 & \frac{l}{6} & 0 & 0 \\
0 & \frac{l}{12} & -\frac{l^{2}}{20} & 0 & -\frac{l}{12} & -\frac{l^{2}}{30} \\
0 & \frac{1}{2} & \frac{7 l}{20} & 0 & \frac{1}{2} & \frac{3 l}{20} \\
\frac{l}{6} & 0 & 0 & \frac{l}{3} & 0 & 0 \\
0 & -\frac{l}{12} & \frac{l^{2}}{30} & 0 & \frac{l}{12} & \frac{l^{2}}{20} \\
0 & -\frac{1}{2} & \frac{3 l}{20} & 0 & -\frac{1}{2} & \frac{7 l}{20}
\end{array}\right]
$$


A matriz (4.64) foi obtida para o sistema local de coordenadas. Esta matriz transformada para o sistema global fica:

$$
\underset{\sim}{N}=\left[\begin{array}{cccccc}
\left(\frac{C_{x}^{2}}{3}+\frac{C_{y}^{2}}{12}\right) l & \frac{l}{4} C_{x} C_{y} & \frac{l^{2}}{20} C_{y} & \left(\frac{C_{x}^{2}}{6}-\frac{C_{y}^{2}}{12}\right) l & \frac{l}{4} C_{x} C_{y} & \frac{l^{2}}{30} C_{y} \\
\frac{l}{4} C_{x} C_{y} & \left(\frac{C_{y}^{2}}{3}+\frac{C_{x}^{2}}{12}\right) l & -\frac{l^{2}}{20} C_{x} & \frac{l}{4} C_{x} C_{y} & \left(\frac{C_{y}^{2}}{6}-\frac{C_{x}^{2}}{12}\right) l & -\frac{l^{2}}{30} C_{x} \\
-\frac{1}{2} C_{y} & \frac{1}{2} C_{x} & \frac{7 l}{20} & -\frac{1}{2} C_{y} & \frac{1}{2} C_{x} & \frac{3 l}{20} \\
\left(\frac{C_{x}^{2}}{6}-\frac{C_{y}^{2}}{12}\right) l & \frac{l}{4} C_{x} C_{y} & -\frac{l^{2}}{30} C_{y} & \left(\frac{C_{x}^{2}}{3}+\frac{C_{y}^{2}}{12}\right) l & \frac{l}{4} C_{x} C_{y} & -\frac{l^{2}}{20} C_{y} \\
\frac{l}{4} C_{x} C_{y} & \left(\frac{C_{y}^{2}}{6}-\frac{C_{x}^{2}}{12}\right) l & \frac{l^{2}}{30} C_{x} & \frac{l}{4} C_{x} C_{y} & \left(\frac{C_{y}^{2}}{3}+\frac{C_{x}^{2}}{12}\right) l & \frac{l^{2}}{20} C_{x} \\
\frac{1}{2} C_{y} & -\frac{1}{2} C_{x} & \frac{3 l}{20} & \frac{1}{2} C_{y} & -\frac{1}{2} C_{x} & \frac{7 l}{20}
\end{array}\right]
$$

O elemento genérico da ligação placa-pilar, que foi dado na Fig. 4.6, tem 3 nós e pode ser interpretado como a associação de dois elementos de barra, cujas matrizes " $k_{e}$ " e " $N$ " para o sistema de coordenadas global estão expressas respectivamente em (4.59) e (4.65). Esta situação representada por um elemento de carga correspondente a dois elementos de barra está ilustrada na Fig. 4.9, onde se mostra o novo sistema de coordenadas locais, agora com 9 coordenadas. 


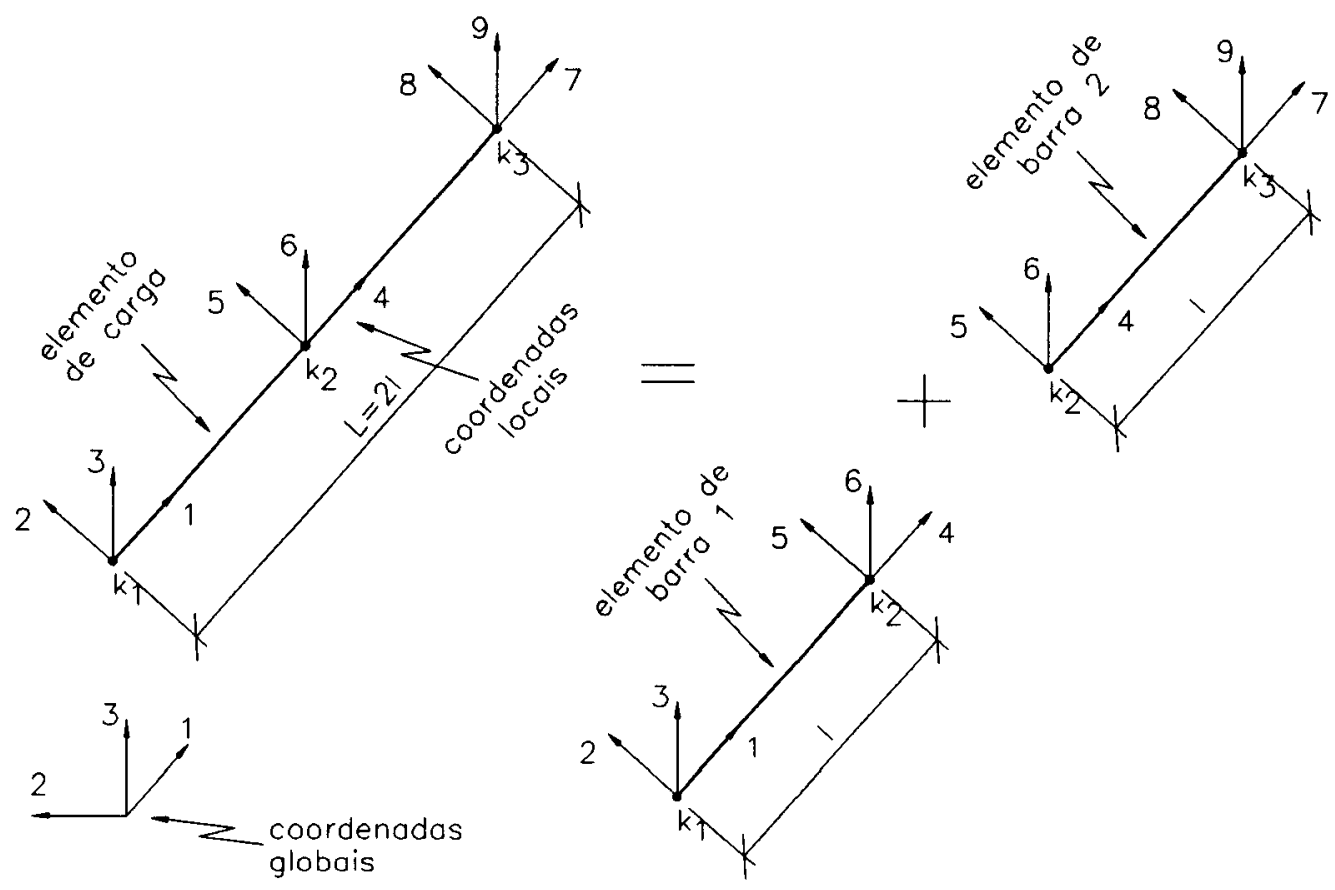

Fig. 4.9 - Elementos de carga e de barra.

A matriz " $k_{\varepsilon}$ " no sistema global para o elemento de carga será dada por:

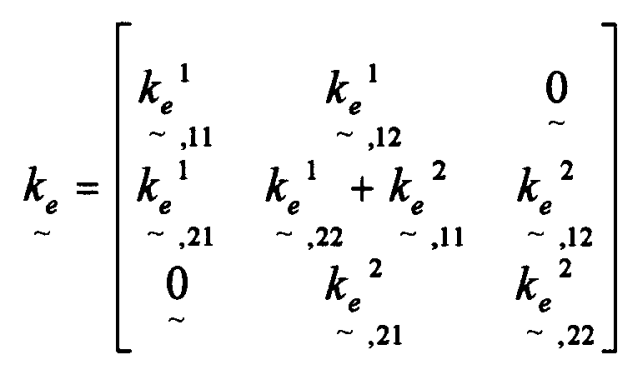

onde:

$$
\underset{\sim, 11}{k_{e}{ }^{1}=}=\left[\begin{array}{ccc}
2 \frac{G J_{t}}{L} C_{x}^{2}+\frac{8 E I}{L} C_{y}^{2} & \left(\frac{G J_{t}}{L}-\frac{4 E I}{L}\right) 2 C_{x} C_{y} & \frac{24 E I C_{y}}{L^{2}} \\
\text { S i me'tri c a } & 2 \frac{G J_{t}}{L} C_{y}^{2}+\frac{8 E I}{L} C_{x}^{2} & -\frac{24 E I C_{x}}{L^{2}} \\
\frac{96 E I}{L^{3}}
\end{array}\right]
$$




$$
\underset{\sim, 12}{k_{e}{ }^{1}}=\underset{\sim, 12}{k_{e}{ }^{2}}=\left[\begin{array}{ccc}
-2 \frac{G J_{t}}{L} C_{x}^{2}+\frac{4 E I}{L} C_{y}^{2} & -\left(\frac{G J_{t}}{L}+\frac{2 E I}{L}\right) 2 C_{x} C_{y} & -\frac{24 E I C_{y}}{L^{2}} \\
-\left(\frac{G J_{t}}{L}+\frac{2 E I}{L}\right) 2 C_{x} C_{y} & -2 \frac{G J_{t}}{L} C_{y}^{2}+\frac{4 E I}{L} C_{x}^{2} & \frac{24 E I C_{x}}{L^{2}} \\
\frac{24 E I C_{y}}{L^{2}} & -\frac{24 E I C_{x}}{L^{2}} & -\frac{96 E I}{L^{3}}
\end{array}\right]
$$

(4.67.b )

$$
\underset{\sim, 21}{k_{e}{ }^{1}}=\underset{\sim, 21}{k_{e}{ }^{2}}=\left[\begin{array}{ccc}
-2 \frac{G J_{t}}{L} C_{x}^{2}+\frac{4 E I}{L} C_{y}^{2} & -\left(\frac{G J_{t}}{L}+\frac{2 E I}{L}\right) 2 C_{x} C_{y} & \frac{24 E I C_{y}}{L^{2}} \\
-\left(\frac{G J_{t}}{L}+\frac{2 E I}{L}\right) 2 C_{x} C_{y} & -2 \frac{G J_{t}}{L} C_{y}^{2}+\frac{4 E I}{L} C_{x}^{2} & -\frac{24 E I C_{x}}{L^{2}} \\
-\frac{24 E I C_{y}}{L^{2}} & \frac{24 E I C_{x}}{L^{2}} & -\frac{96 E I}{L^{3}}
\end{array}\right]
$$

(4.67.c)

$$
\sim_{, 22}^{k^{1}+\dot{k}_{e}{ }^{2}}=\left[\begin{array}{ccc}
4 \frac{G J_{t}}{L} C_{x}^{2}+\frac{16 E I}{L} C_{y}^{2} & \left(\frac{G J_{t}}{L}-\frac{4 E I}{L}\right) 4 C_{x} C_{y} & 0 \\
\text { Si me't ri c a } & 4 \frac{G J_{t}}{L} C_{y}^{2}+\frac{16 E I}{L} C_{x}^{2} & 0 \\
& \frac{192 E I}{L^{3}}
\end{array}\right]
$$

$$
\underset{\sim, 22}{k_{e}{ }^{2}}=\left[\begin{array}{ccc}
2 \frac{G J_{t}}{L} C_{x}^{2}+\frac{8 E I}{L} C_{y}^{2} & \left(\frac{G J_{t}}{L}-\frac{4 E I}{L}\right) 2 C_{x} C_{y} & -\frac{24 E I}{L^{2}} C_{y} \\
\text { Sime'tric a } & 2 \frac{G J_{t}}{L} C_{y}^{2}+\frac{8 E I}{L} C_{x}^{2} & \frac{24 E I}{L^{2}} C_{x} \\
\frac{96 E I}{L^{3}}
\end{array}\right] \text { (4.67.e) }
$$

onde " $L$ " é o comprimento do elemento de carga, que é igual a duas vezes o comprimento do elemento de barra "l".

De maneira análoga, a matriz " $N$ " no sistema global de coordenadas, para o elemento de carga, será dada por: 


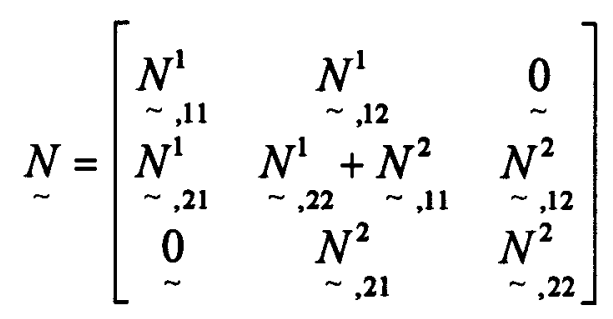

onde:

$$
\begin{aligned}
& {\left[\left(\frac{C_{x}^{2}}{6}+\frac{C_{y}^{2}}{24}\right) L \quad \frac{L}{8} C_{x} C_{y} \quad \frac{L^{2}}{80} C_{y}\right.} \\
& \underset{\sim 11}{N^{1}}=\frac{L}{8} C_{x} C_{y} \quad\left(\frac{C_{x}^{2}}{24}+\frac{C_{y}^{2}}{6}\right) L-\frac{L^{2}}{80} C_{x} \\
& \begin{array}{lll}
-\frac{1}{2} C_{y} & \frac{1}{2} C_{x} & \frac{7 L}{40}
\end{array} \\
& {\left[\left(\frac{C_{x}^{2}}{12}-\frac{C_{y}^{2}}{24}\right) L \quad \frac{L}{8} C_{x} C_{y} \quad \frac{L^{2}}{120} C_{y}\right.} \\
& \underset{\sim 12}{N_{1}^{1}}=\underset{\sim 12}{N^{2}}=\mid \frac{L}{8} C_{x} C_{y} \quad\left(\frac{C_{y}^{2}}{12}-\frac{C_{x}^{2}}{24}\right) L-\frac{L^{2}}{120} C_{x} \\
& \begin{array}{lll}
-\frac{1}{2} C_{y} & \frac{1}{2} C_{x} & \frac{3 L}{40}
\end{array} \\
& \underset{\sim 21}{N^{1}}=\underset{\sim 21}{N_{2}^{2}}=\left[\begin{array}{ccc}
\left(\frac{C_{x}^{2}}{12}-\frac{C_{y}^{2}}{24}\right) L & \frac{L}{8} C_{x} C_{y} & -\frac{L^{2}}{120} C_{y} \\
\frac{L}{8} C_{x} C_{y} & \left(\frac{C_{y}^{2}}{12}-\frac{C_{x}^{2}}{24}\right) L & \frac{L^{2}}{120} C_{x} \\
\frac{1}{2} C_{y} & -\frac{1}{2} C_{x} & \frac{3 L}{40}
\end{array}\right]
\end{aligned}
$$

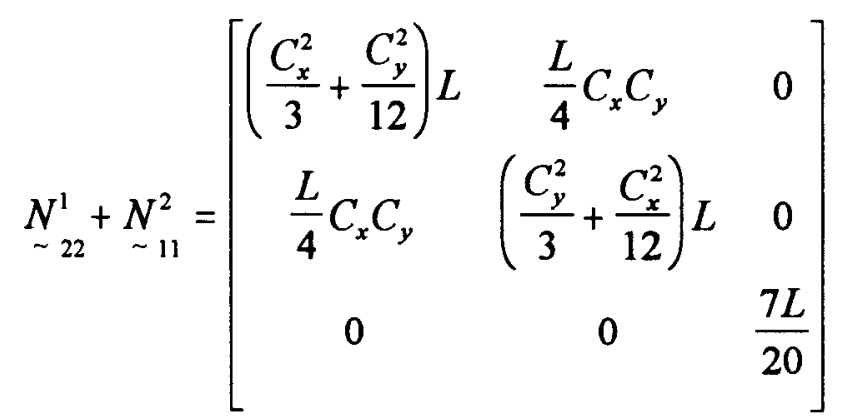




$$
\underset{\sim 22}{N_{2}^{2}}=\left[\begin{array}{ccc}
\left(\frac{C_{x}^{2}}{6}+\frac{C_{y}^{2}}{24}\right) L & \frac{L}{8} C_{x} C_{y} & -\frac{L^{2}}{80} C_{y} \\
\frac{L}{8} C_{x} C_{y} & \left(\frac{C_{y}^{2}}{6}+\frac{C_{x}^{2}}{24}\right) L & \frac{L^{2}}{80} C_{x} \\
\frac{1}{2} C_{y} & -\frac{1}{2} C_{x} & \frac{7 L}{40}
\end{array}\right]
$$




\section{4 - FLUXOGRAMAS}

São apresentados a seguir os fluxogramas do programa principal e de algumas subrotinas de forma bem compacta.

FLUXOGRAMA GERAL

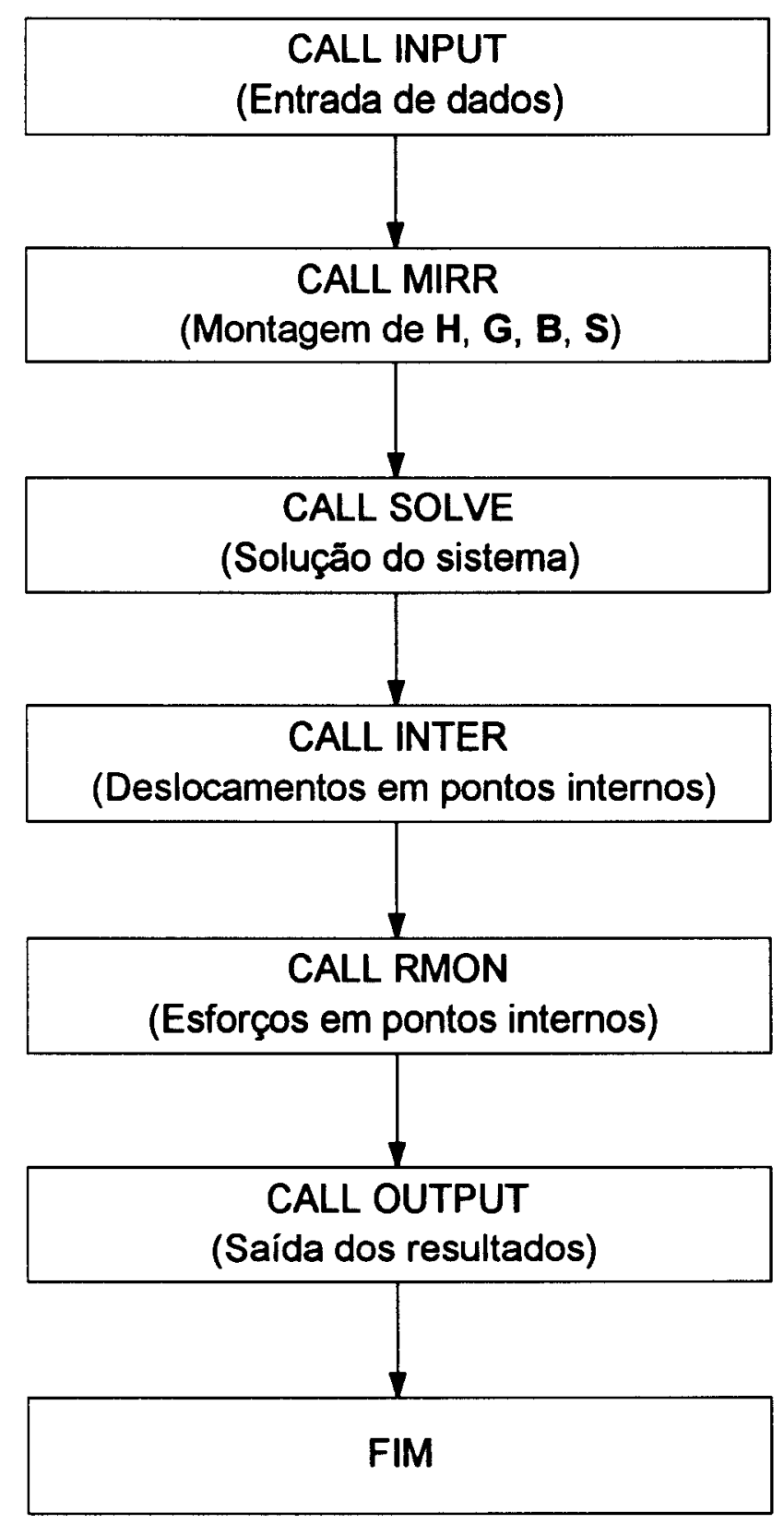




\section{SUBROUTINE INPUT}

\section{Subrotina para entrada de dados}

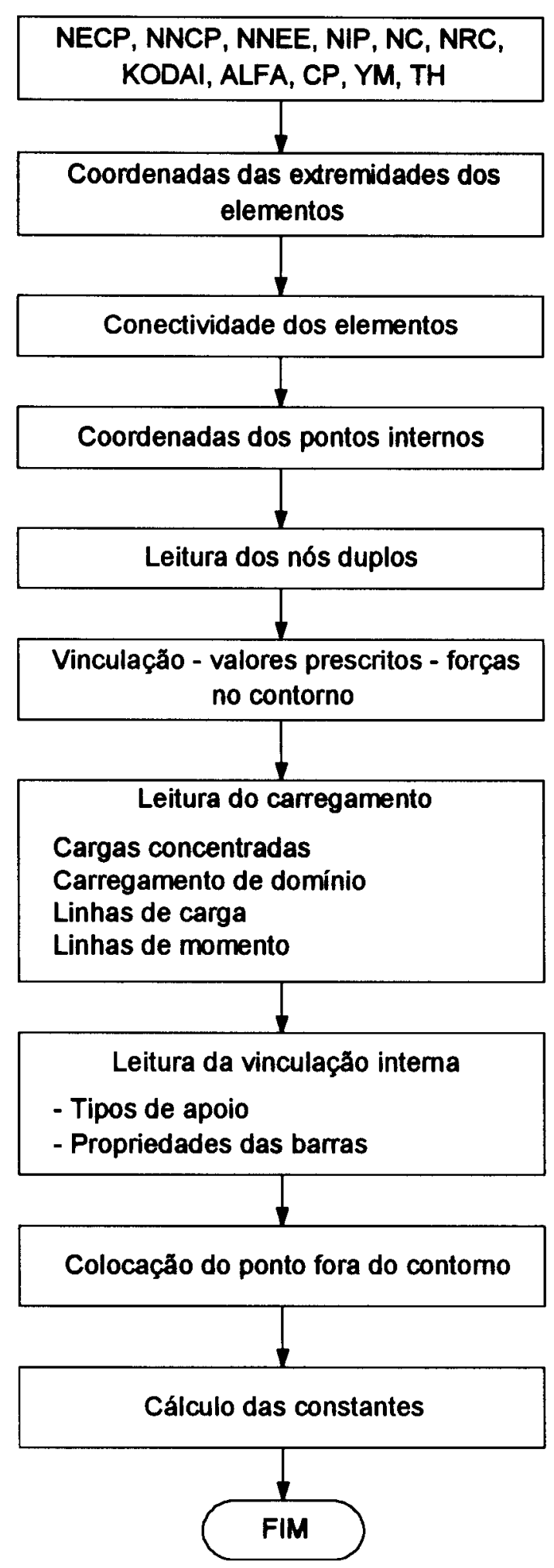

- NECP, número de elementos do contorno d placa

- NNCP, número de nós do contorno da placa - NNEE, número de nós de extremidade dos elementos

- NIP, número de pontos internos

- NC, número de cantos

- NRC, número de regióes carregadas

- KODAl, código que define as condições de vinculaçăo interna

- ALFA, coeficiente a ser multiplicado pelo comprimento do elemento

- CP, coeficiente de Poison

- YM, módulo de Young do material

- TH, espessura da placa 


\section{SUBROUTINE MIRR}

Subrotina para montagem das matrizes $H$ e $G$, do vetor do carregamento de dominio $\mathbf{B}$ e da matriz $\mathbf{S}$ de influencia das linhas de carga

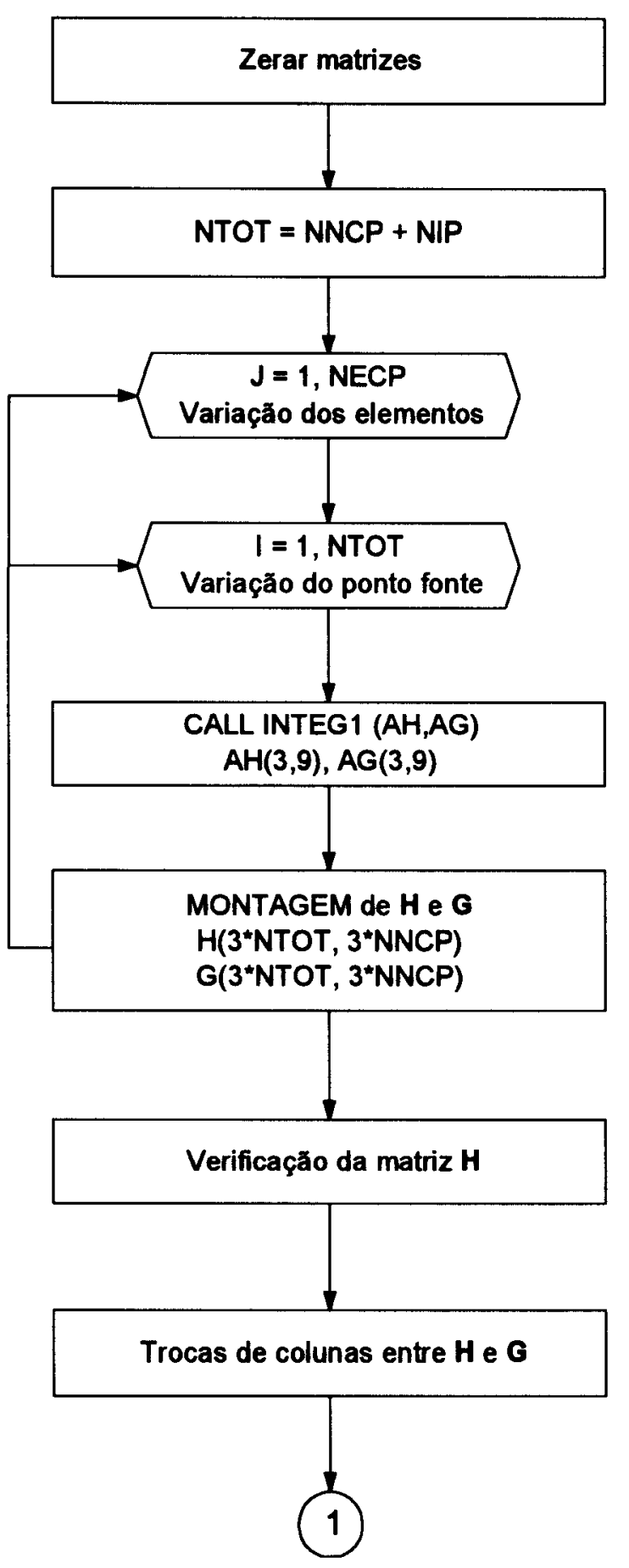

- INTEG1, subrotina que calcula os coeficientes de $\mathrm{He} \mathrm{G}$ 


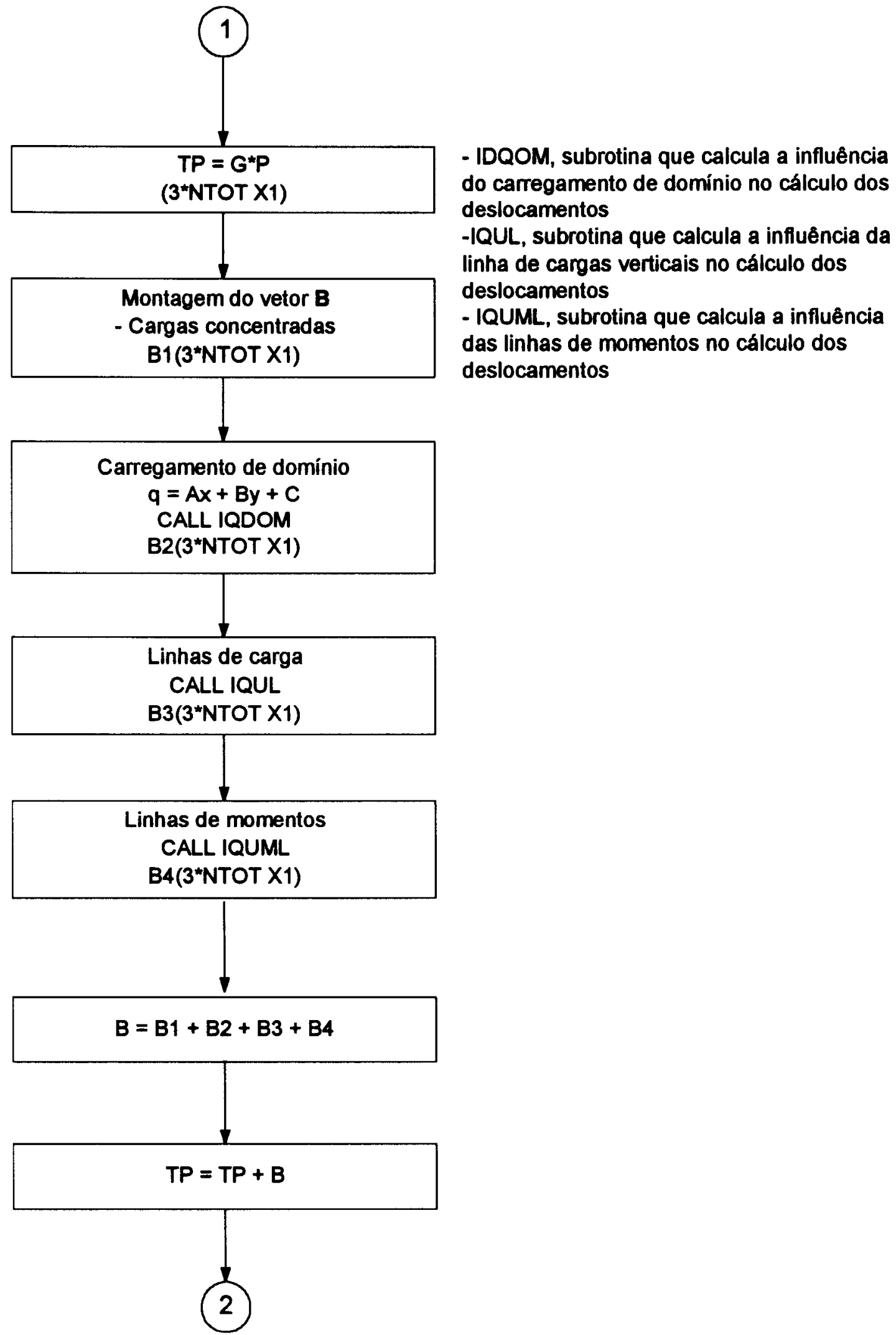




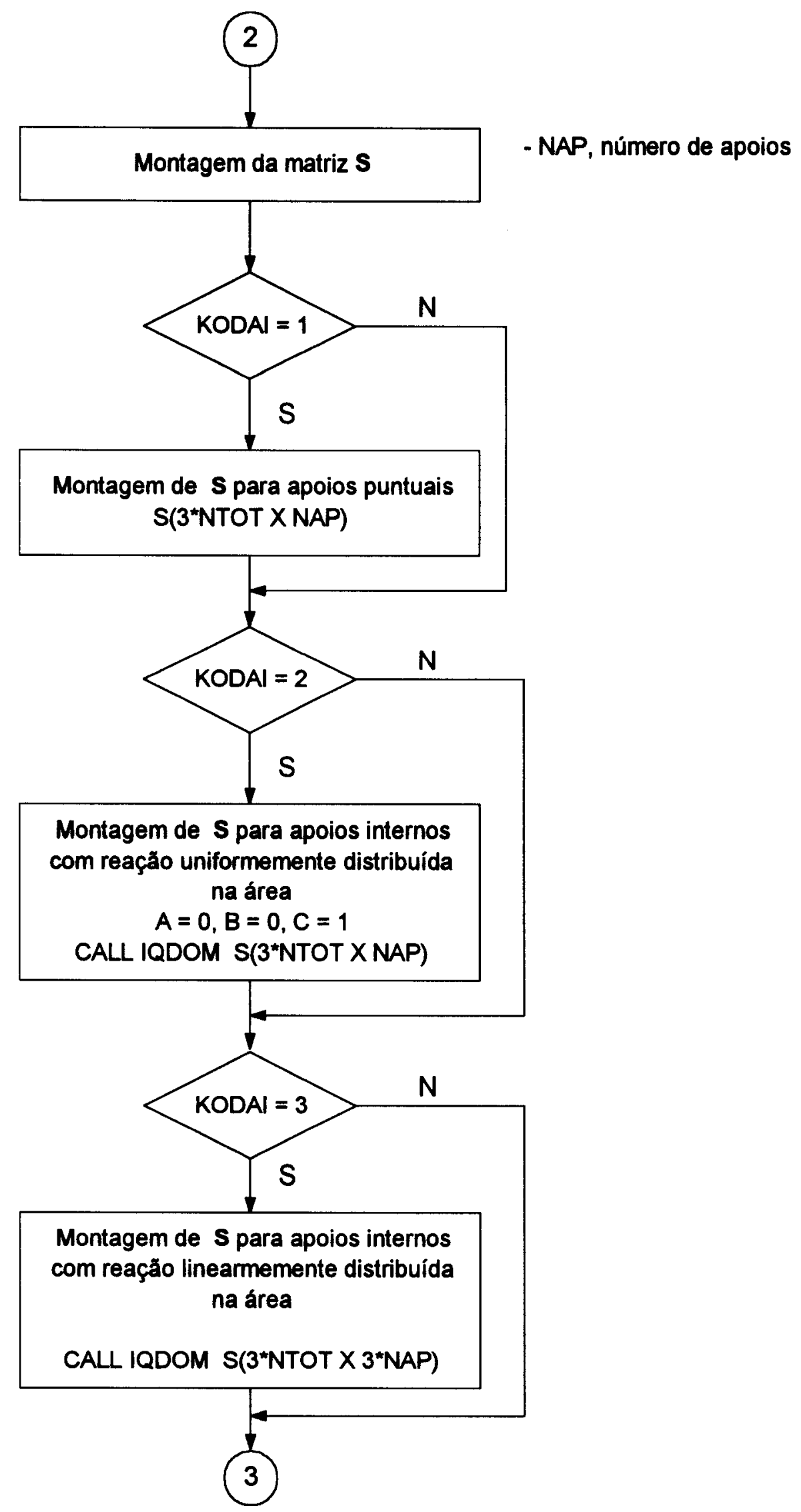




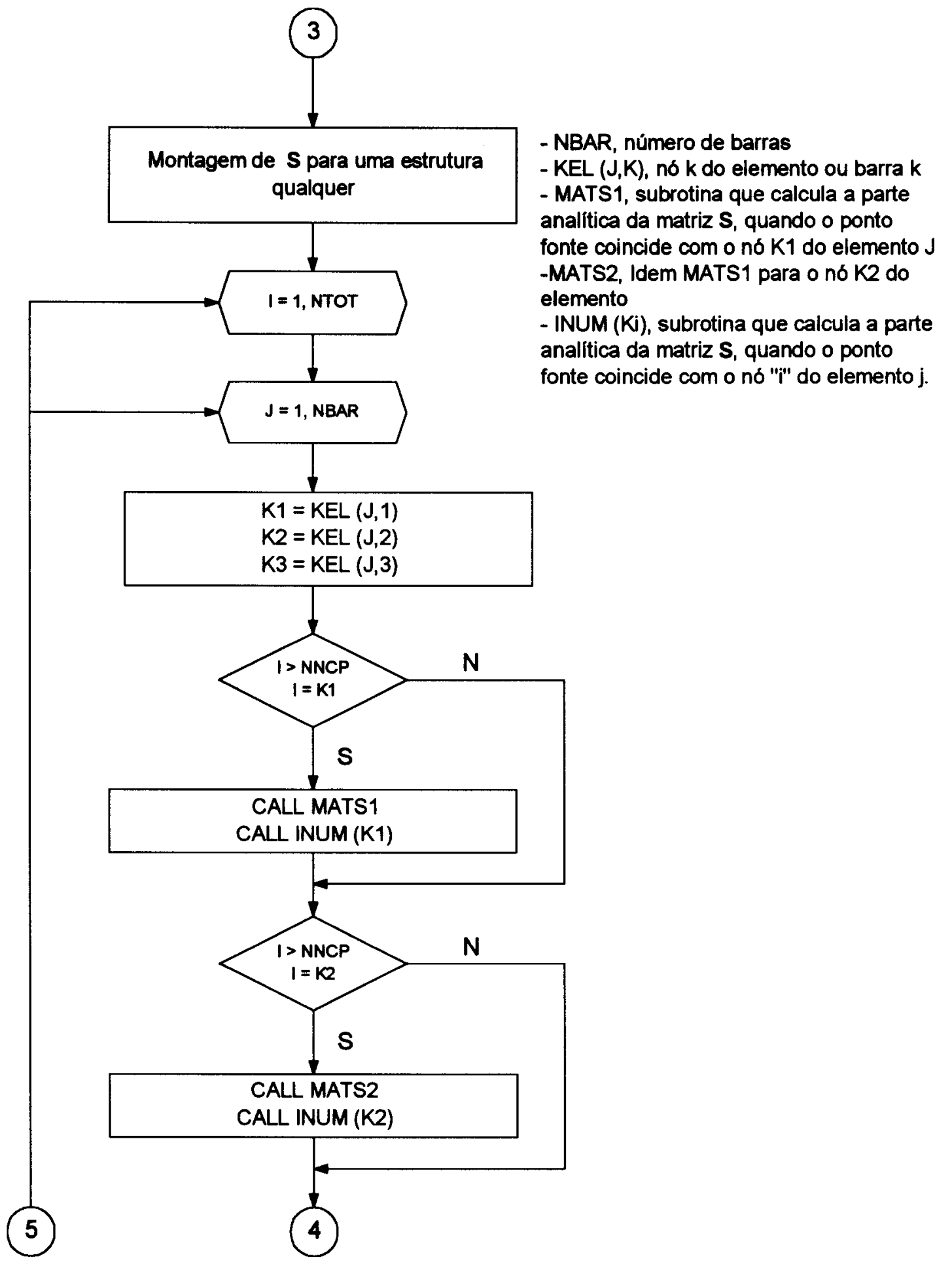




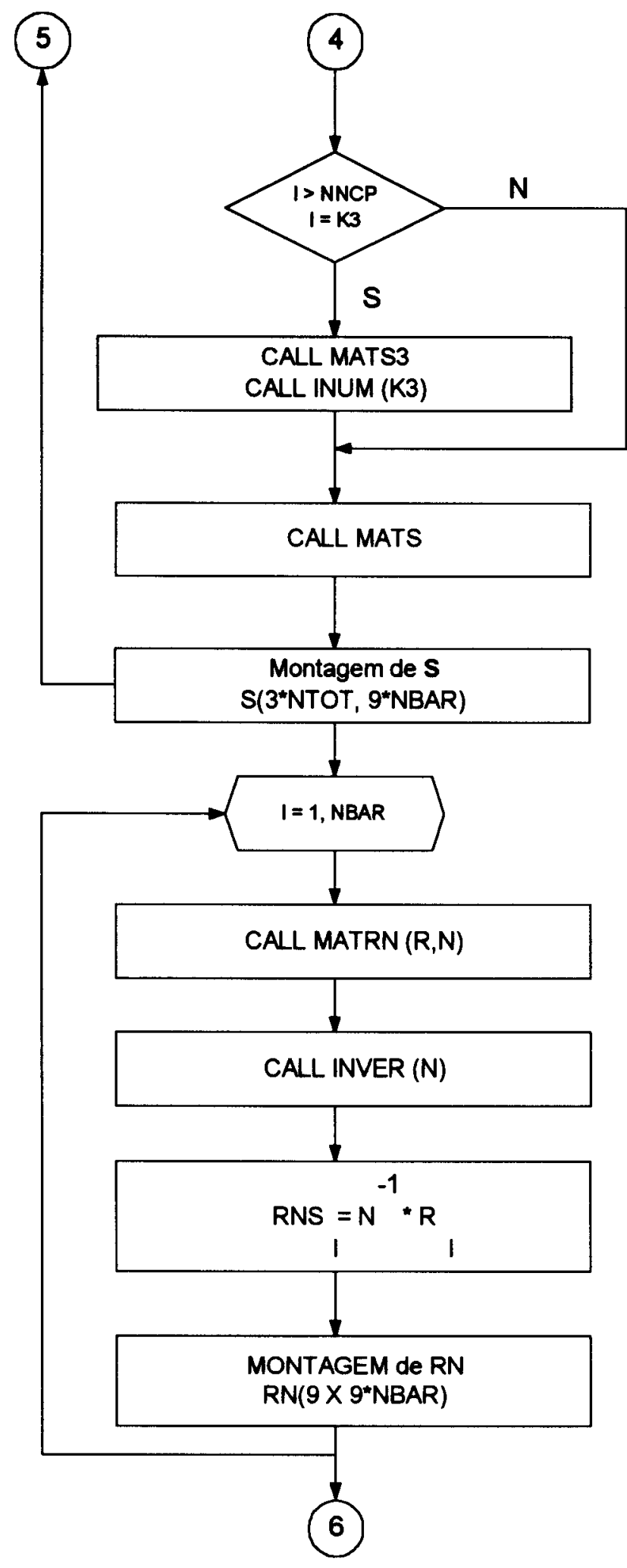

- MATS3, idem MATS1 para o nó K3 do elemento $\mathrm{J}$

- MATS, subrotina que calcula a matriz $\mathbf{S}$ para pontos que náo pertençam à linha de carga

- Cada barra "J" ocupa 9 colunas da matriz S

- MATRN, subrotina que calcula a matri de rigidez $\mathbf{R}$ e a matriz $\mathbf{N}$ do elemento I -INVER, subrotina que inverte a matriz do elemento 1

- RNS, [9X9]

- Cada barra I ocupa 9 colunas da matri RN 


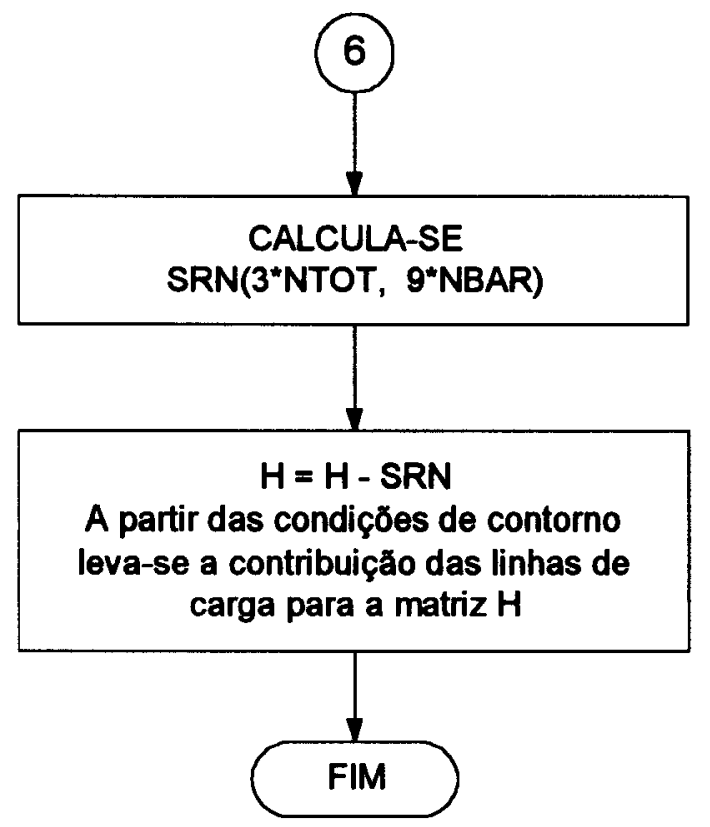

- O produto $S^{*} 1 / N^{*} R$ para cada barra ocupa 9 colunas da matriz SRN 
SUBROUTINE INTER

Calcula os deslocamentos dos pontos internos

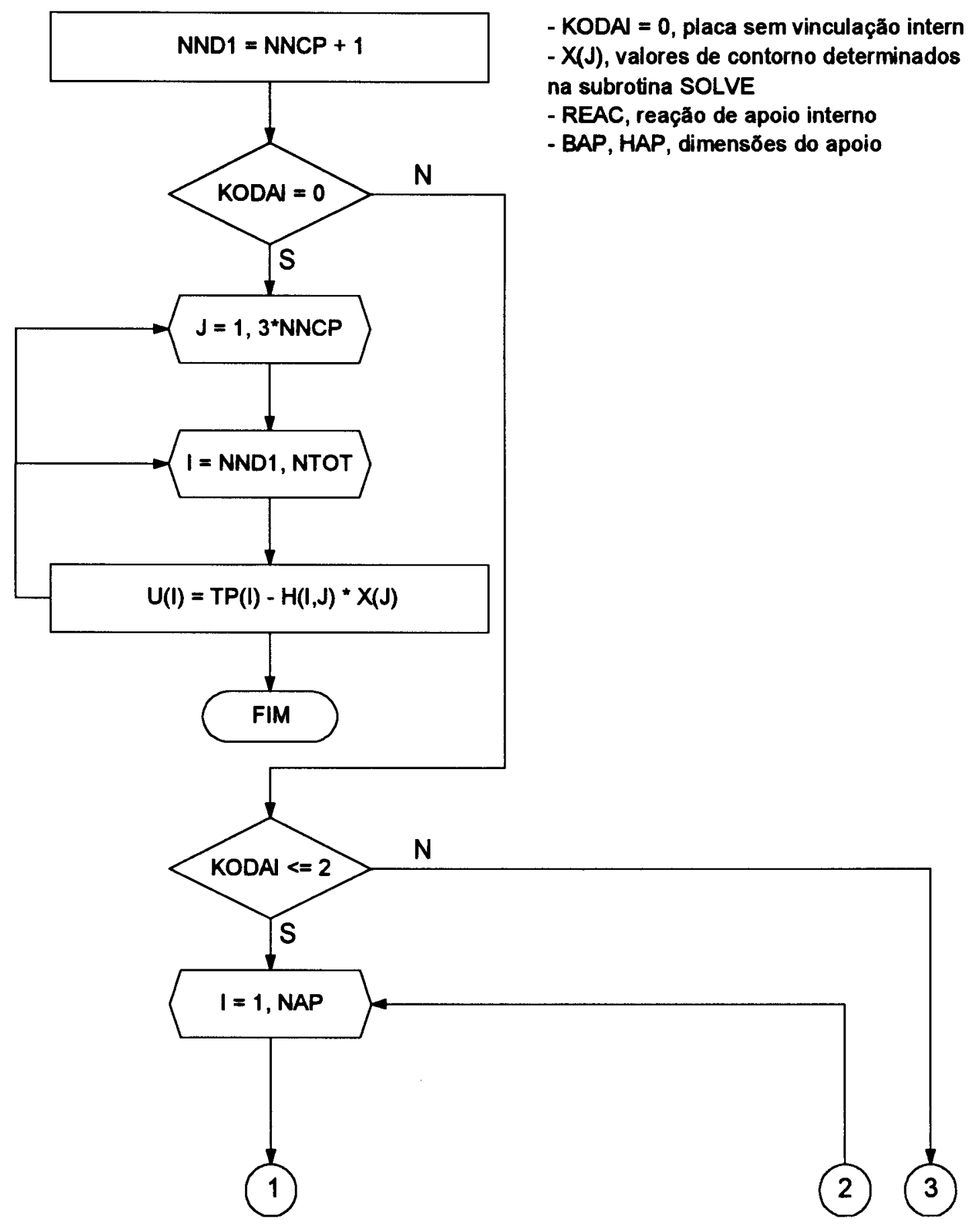

- KODA $=0$, placa sem vinculação intern - X(J), valores de contorno determinados na subrotina SOLVE

- REAC, reação de apoio interno

- BAP, HAP, dimensठ́es do apoio 


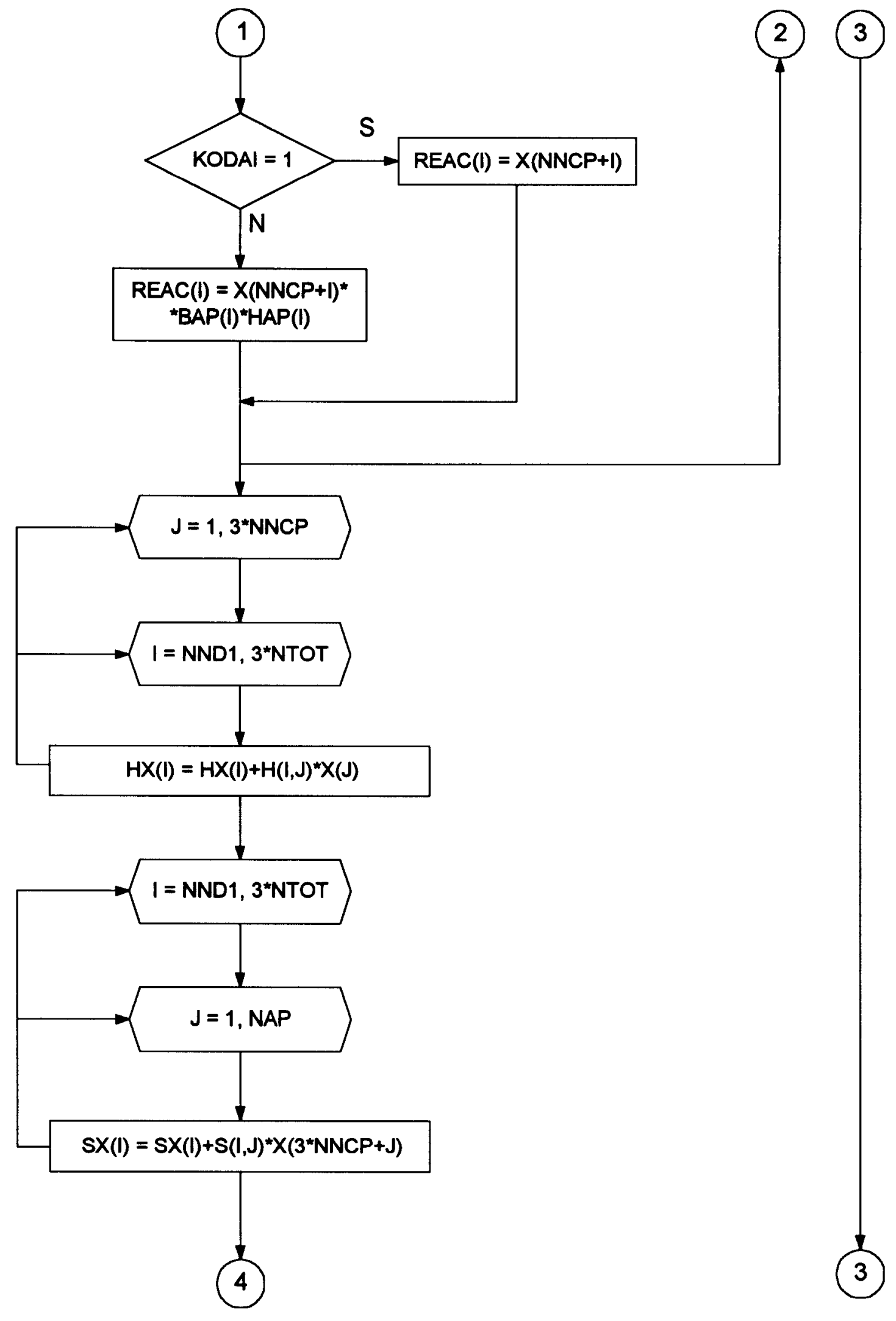




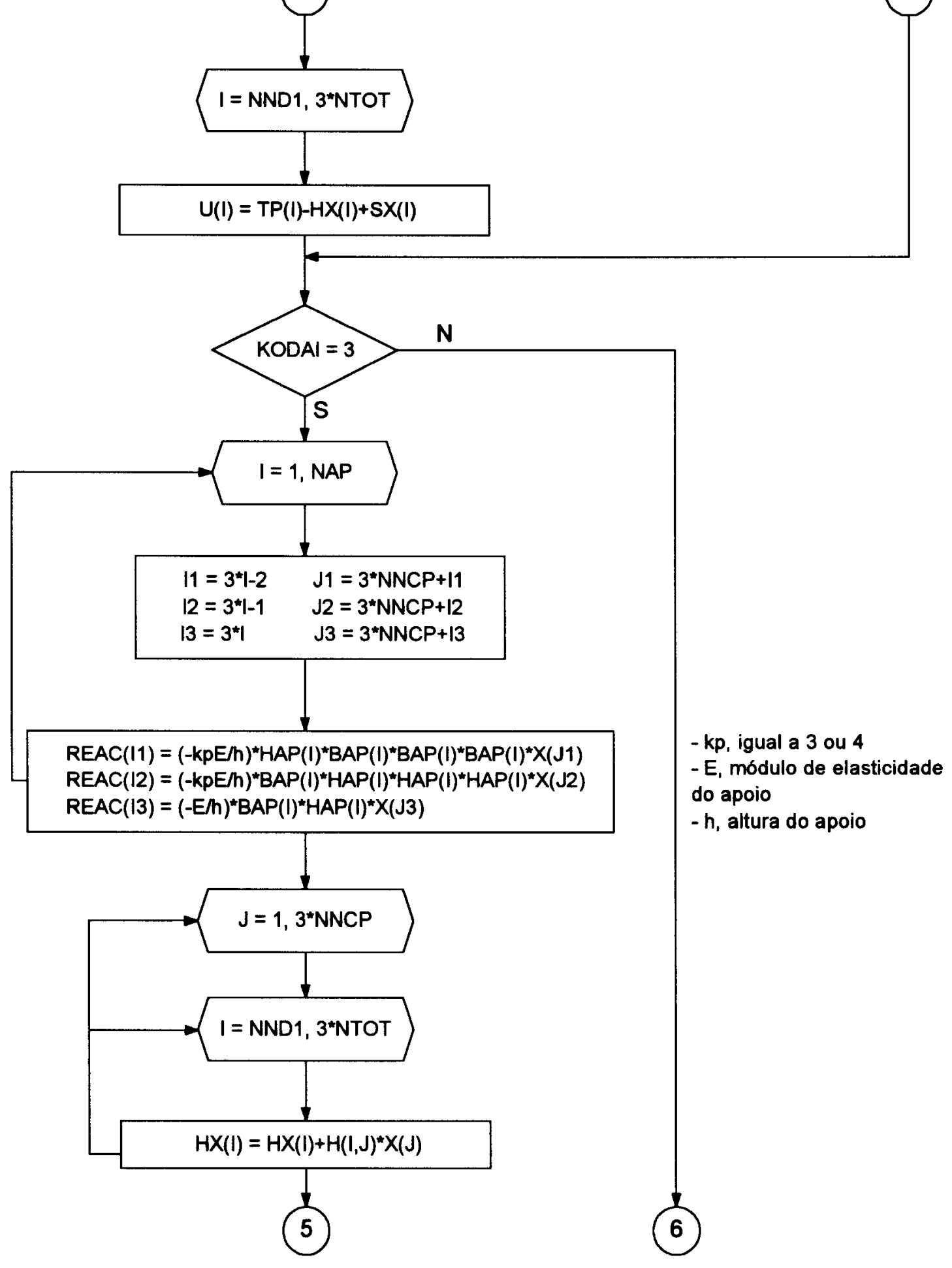




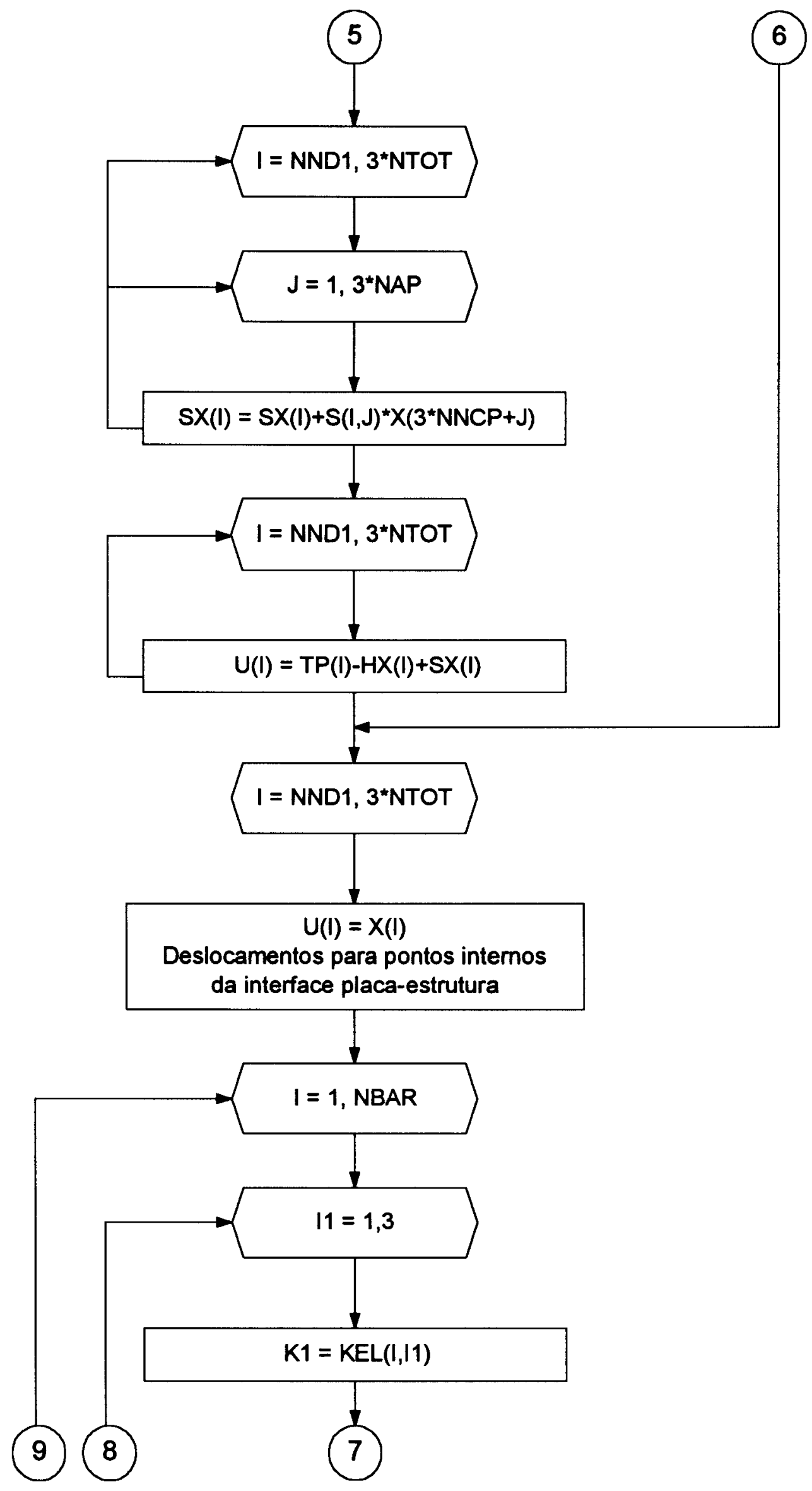




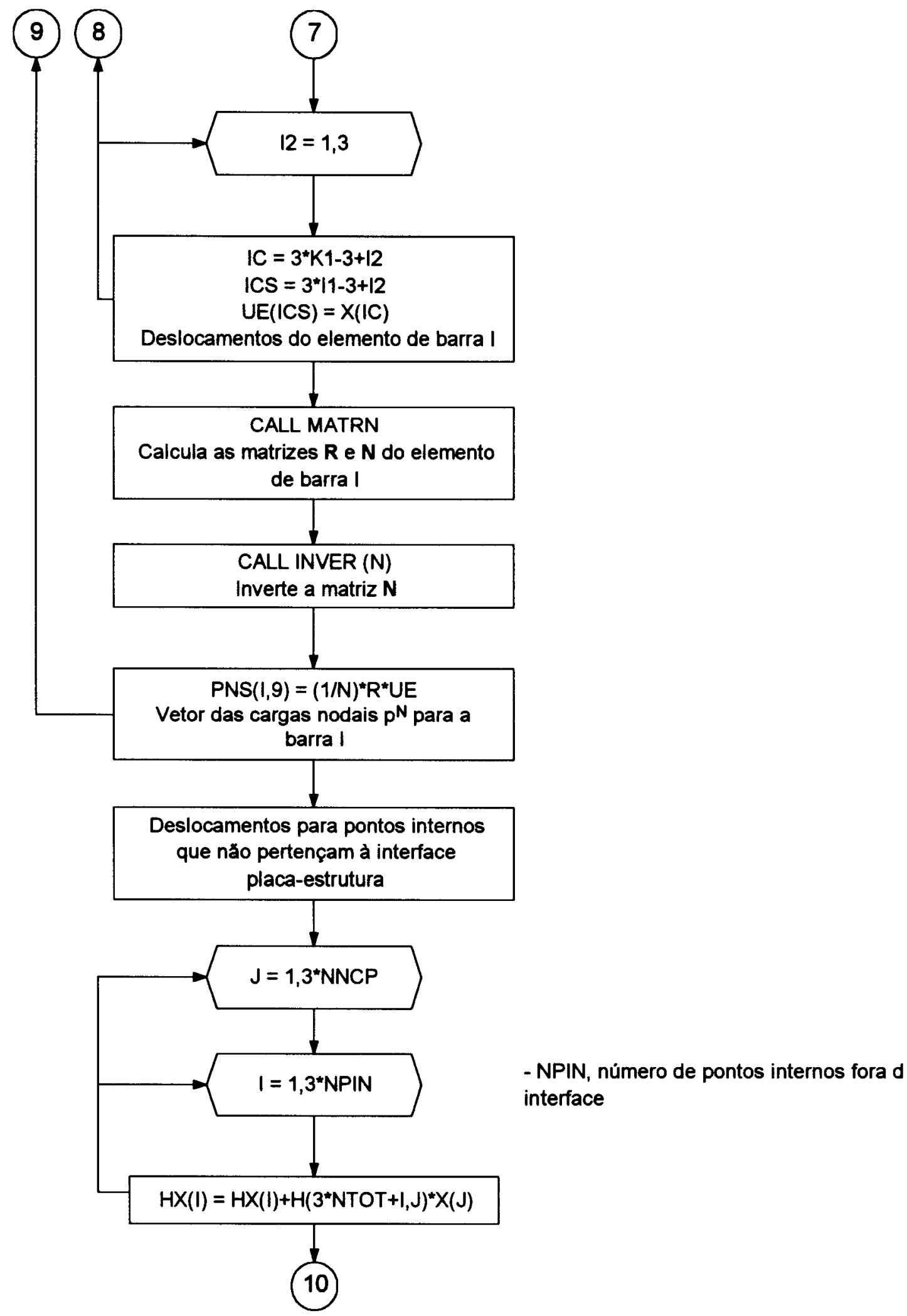




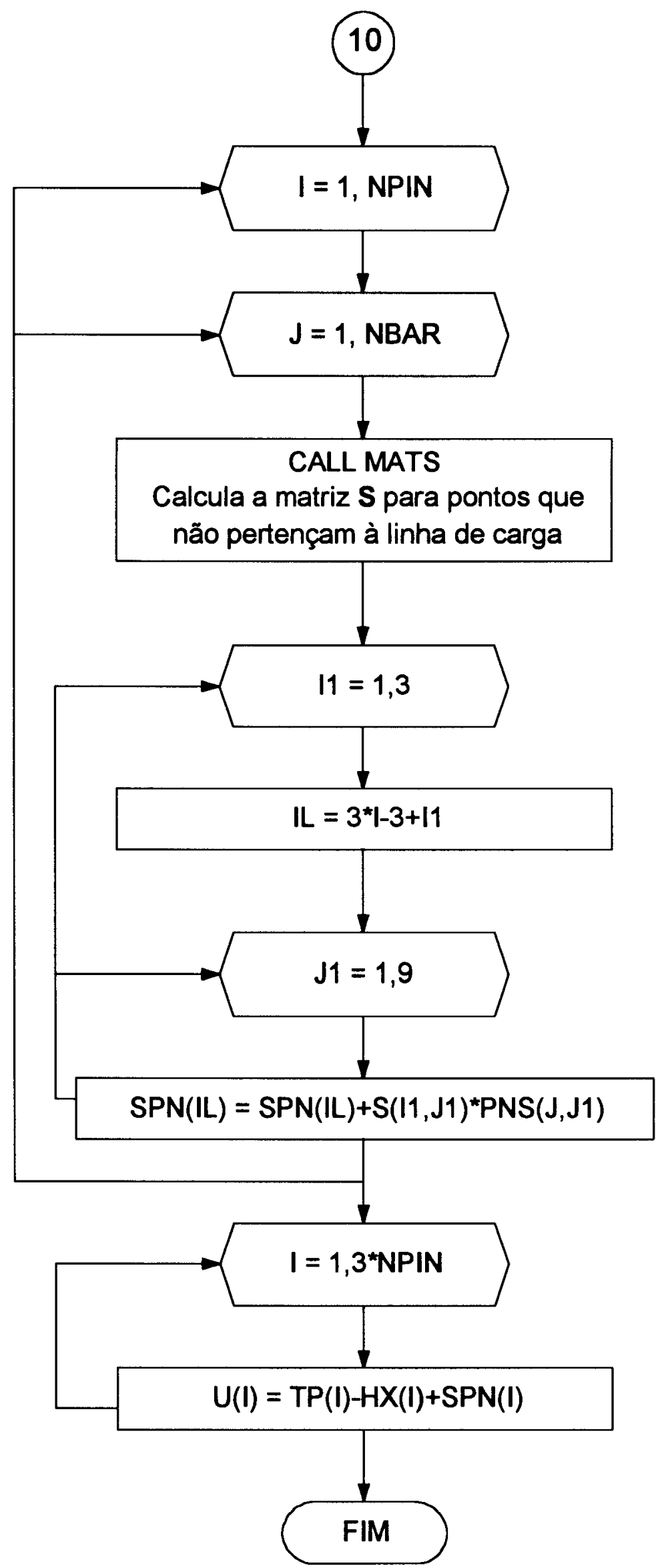




\section{SUBROUTINE RMOM}

Calcula os momentos Mxx, Mxy, Myy e as forças cortantes Qx e Qy em pontos interno

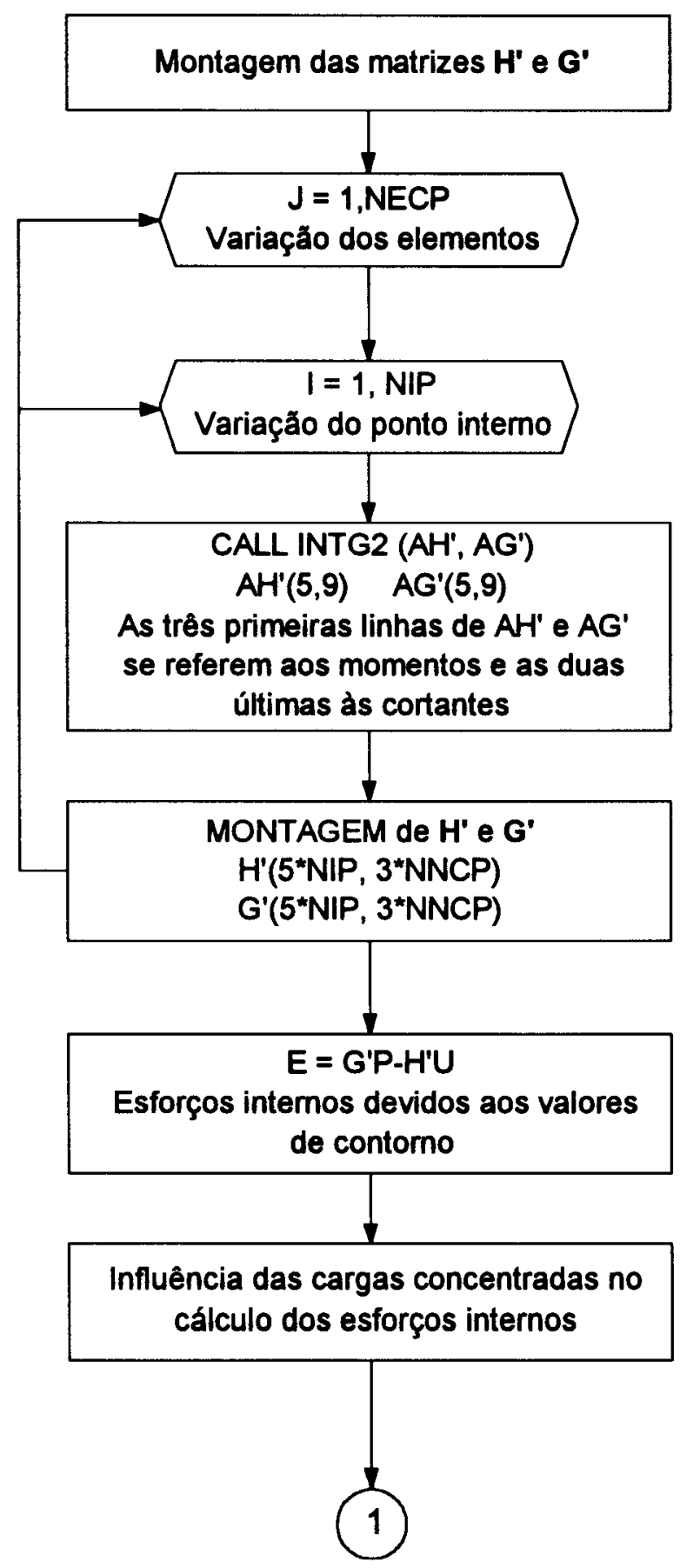




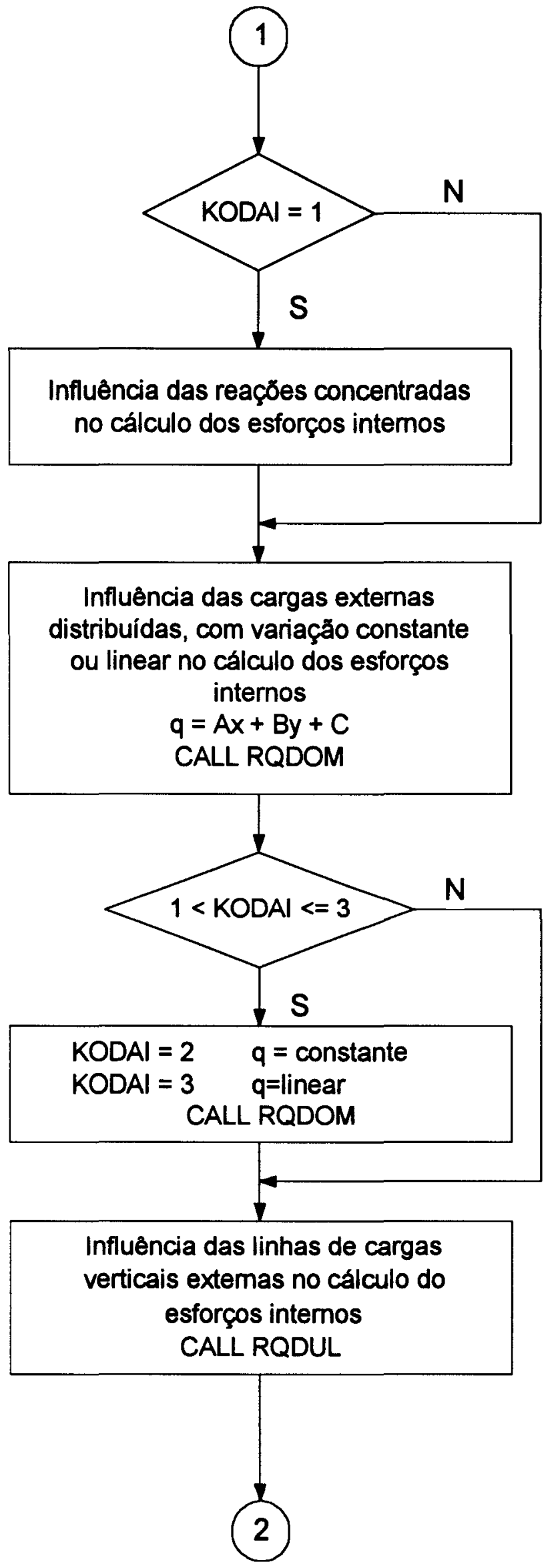




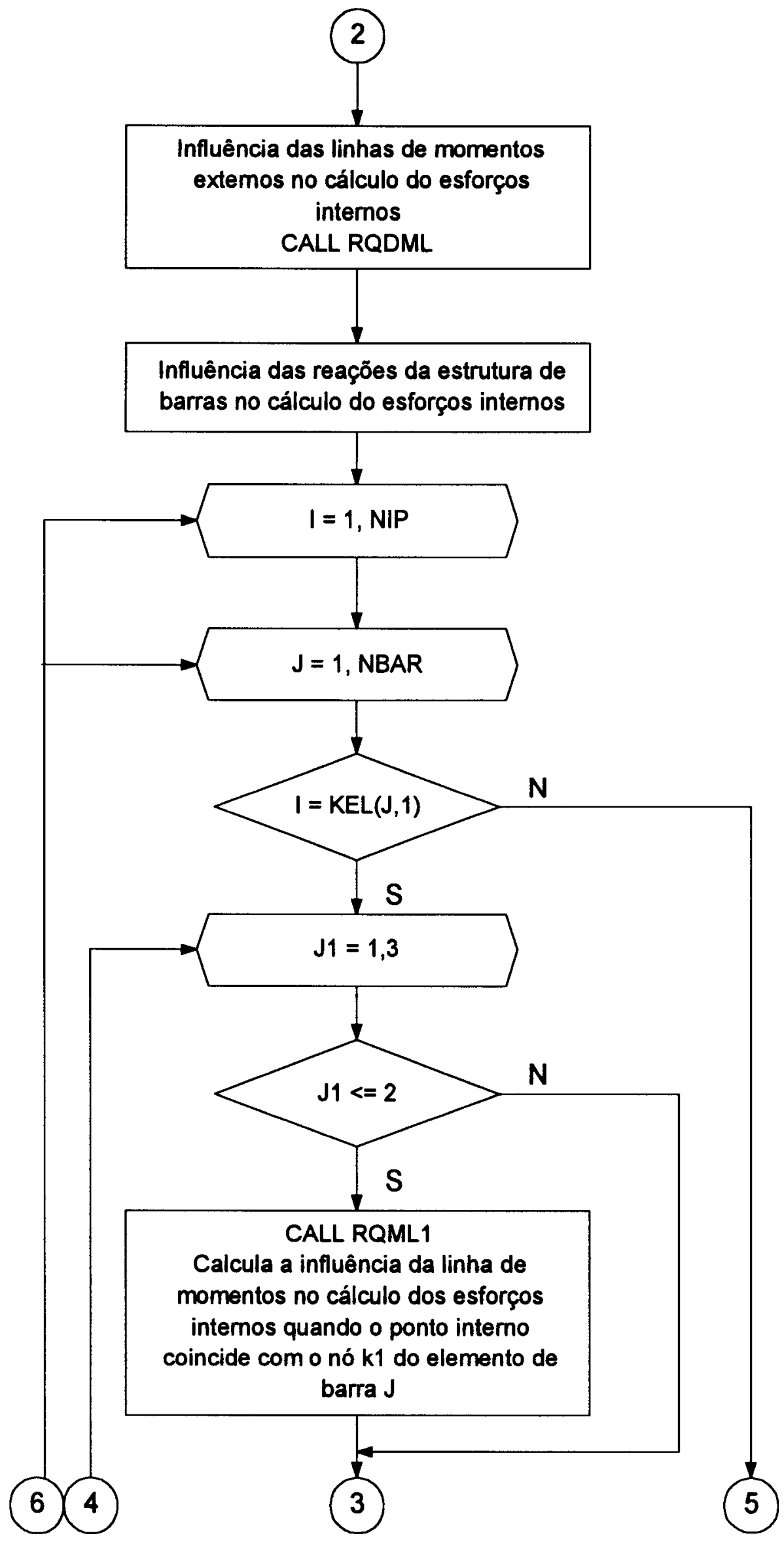




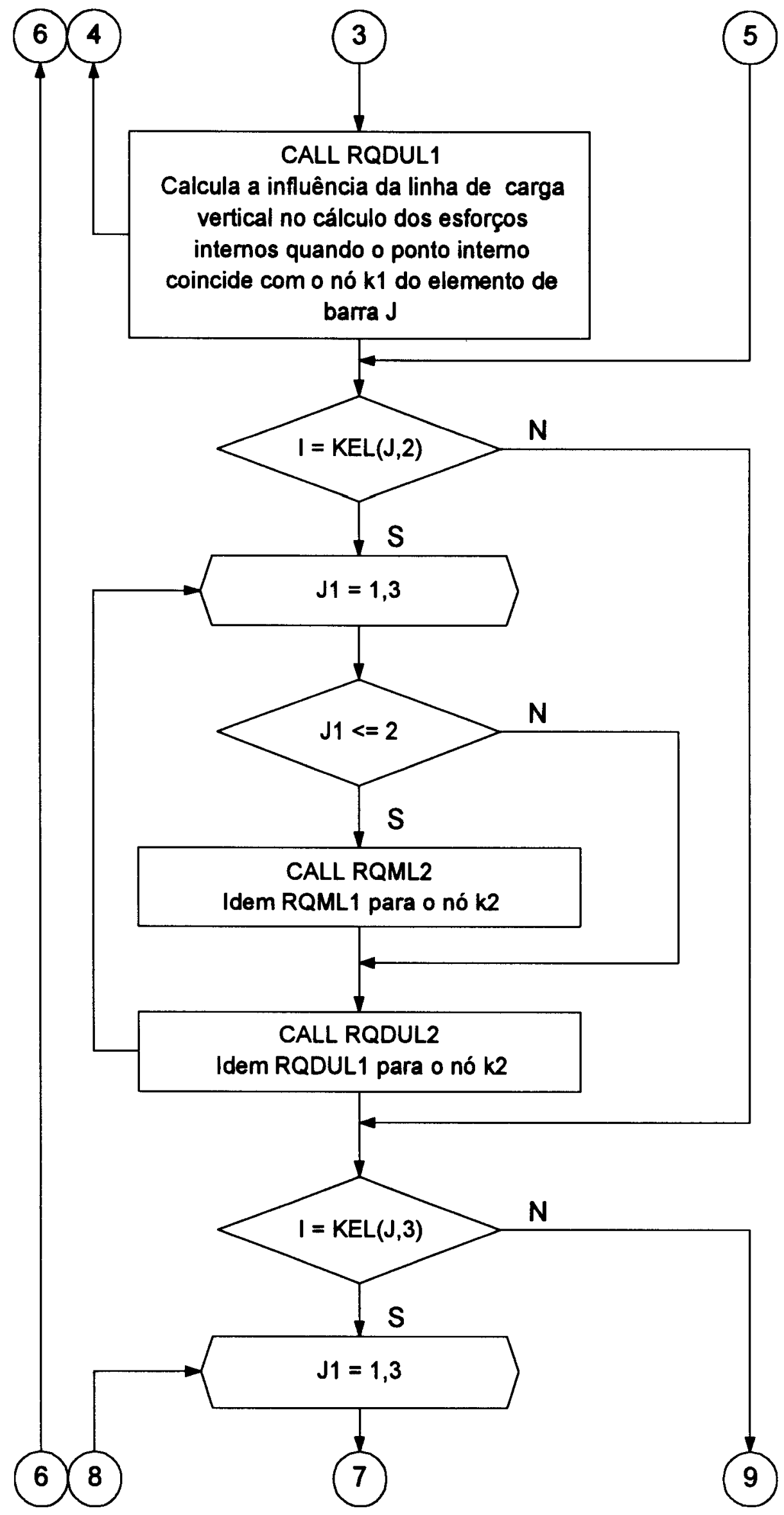




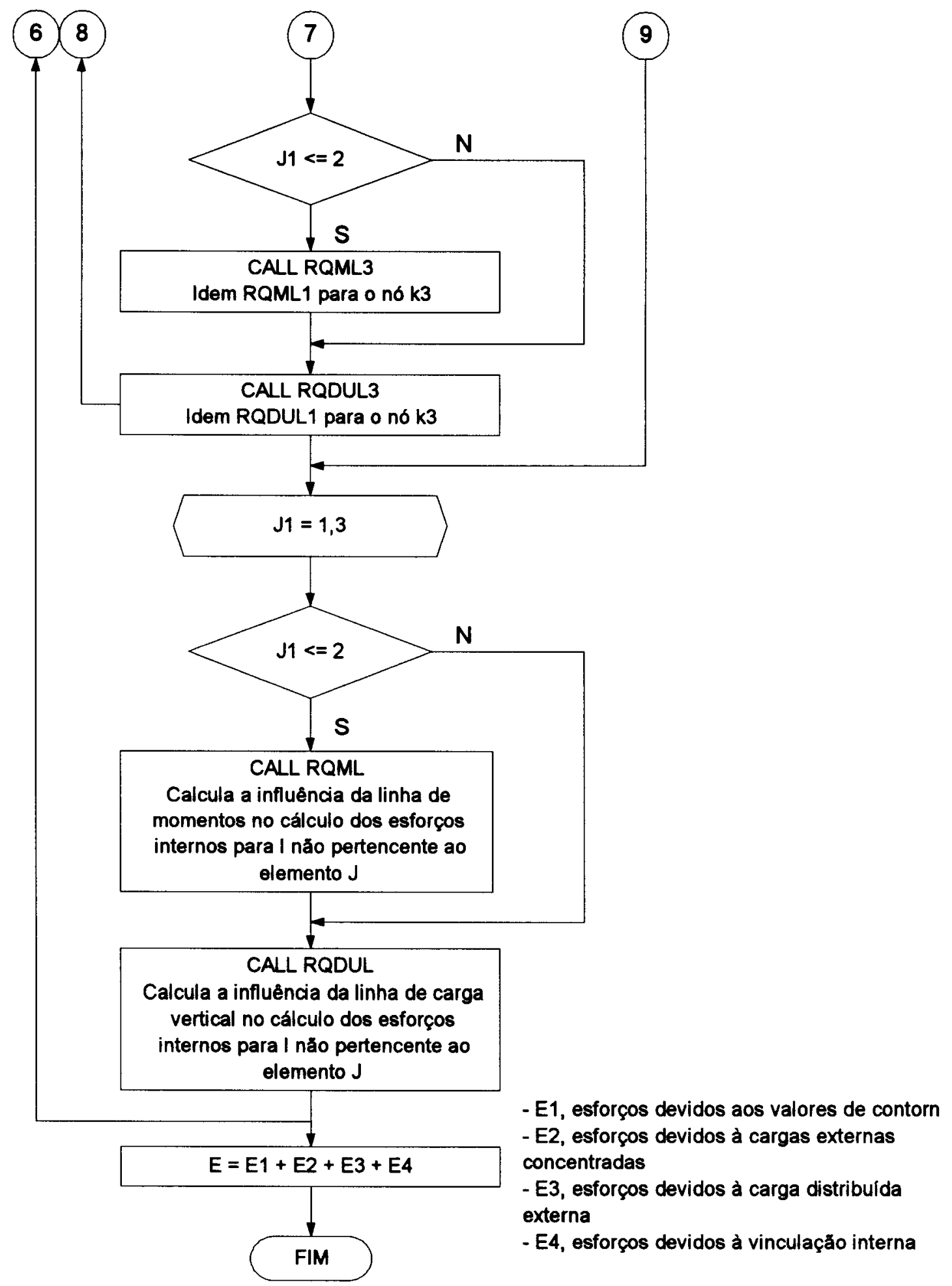




\section{5 - APLICAÇÕES NUMÉRICAS}

São apresentados neste item exemplos de placas resolvidas através do Método dos Elementos de Contorno, utilizando-se a Teoria de Reissner. Os resultados obtidos com o programa desenvolvido neste trabalho, são comparados quando for o caso, com soluções analíticas desenvolvidas por TIMOSHENKO [156], ou com soluçōes numéricas via MEC, apresentadas por PAIVA [113] e RIBEIRO [140], que utilizam respectivamente a teoria clássica e a Teoria de Reissner. Alguns resultados levando em conta a influência da espessura da placa, são comparados com as soluções analíticas obtidas por RIBEIRO [158], através de desenvolvimento em séries.

A eficiência do Método dos Elementos de Contorno aplicado à análise elástica de placas pela Teoria de Reissner já foi comprovada no trabalho de RIBEIRO [140]. Apenas o primeiro exemplo deste item apresentará placas sem vinculação interna, no intuito de mostrar a influência do fator " $\alpha$ ", que quantifica a distância do ponto de colocação para os nós de contorno, no cálculo dos deslocamentos e esforços na placa.

4.5.1 - EXEMPLO 1: Placa quadrada uniformemente carregada.

Neste exemplo analisa-se uma placa quadrada simplesmente apoiada em todo o contorno, submetida a um carregamento uniformemente distribuído. $O$ contorno é discretizado em 20 elementos e são considerados 9 pontos internos, conforme a Fig. 4.10. A discretização do contorno da região carregada, que neste caso é toda a placa, é feita considerando-se 8 elementos. A condição de contorno relativa à rotação tangencial é a "hard condition", ou seja, rotação nula. 


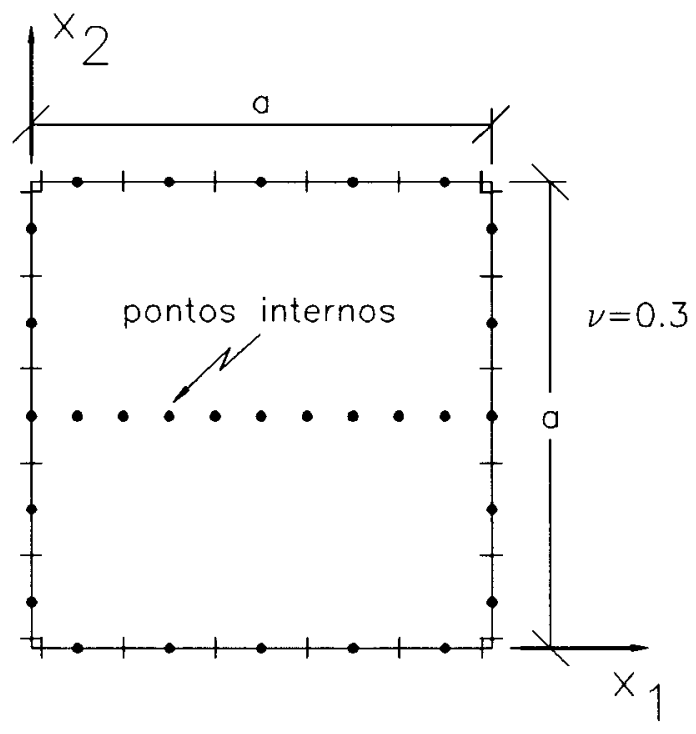

Fig. 4.10 - Placa simplesmente apoiada no contorno.

A tabela 4.1 fornece os deslocamentos transversais " $W$ " na linha de centro da placa $\left(x_{2}=a / 2\right)$ para diversas relaçōes $(h / a)$ e valores " $\alpha$ ".

Analisando-se os resultados da tabela 4.1 nota-se que para $(h / a)=0,01$, os valores dos deslocamentos obtidos com o MEC utilizando-se a teoria de Reissner, coincidem com os calculados com a teoria clássica.

A medida que a espessura aumenta, os deslocamentos obtidos com a Teoria de Reissner ficam sensivelmente maiores que os da teoria clássica, chegando-se a um acréscimo de aproximadamente $40 \%$ no centro da placa, quando a relação $(h / a)=0,30$. Os resultados dos deslocamentos obtidos com a Teoria de Reissner usando-se o MEC mostram uma precisão bastante satisfatória, quando comparados com os valores obtidos analiticamente por RIBEIRO [158], usando-se também a mesma teoria.

Pode-se observar ainda que para " $\alpha$ " variando desde 0,10 a 1,50 , os resultados dos deslocamentos mostram-se praticamente iguais aos valores analíticos obtidos por RIBEIRO [158]. A rigor, os valores serão tanto melhores quanto mais próximo de "zero" ficar a constante " $\alpha$ ". 
Tabela 4.1 - Deslocamento transversal " $w$ " sobre a linha de centro $\left(x_{2}=a / 2\right)$ da placa

\begin{tabular}{|c|c|c|c|c|c|c|}
\hline \multirow[t]{2}{*}{$\mathrm{h} / \mathrm{a}$} & \multirow[t]{2}{*}{$\alpha$} & \multicolumn{5}{|c|}{$w_{x}\left(100 D / q a^{4}\right)$} \\
\hline & & $0,10 \mathrm{~L}$ & $0,20 \mathrm{~L}$ & $0,30 \mathrm{~L}$ & $0,40 \mathrm{~L}$ & $0,50 \mathrm{~L}$ \\
\hline \multirow{6}{*}{0,01} & 0,10 & 0,13188 & 0,24681 & 0,33433 & 0,38867 & 0,40705 \\
\hline & 0,25 & 0,13162 & 0,24638 & 0,33378 & 0,38881 & 0,40641 \\
\hline & 0,50 & 0,13162 & 0,24639 & 0,33378 & 0,38881 & 0,40641 \\
\hline & 1,00 & 0,13159 & 0,24636 & 0,33376 & 0,38804 & 0,40640 \\
\hline & 1,50 & 0,13256 & 0,24464 & 0,33445 & 0,38827 & 0,40596 \\
\hline & RIBEIRO[158] & 0,132 & 0,246 & 0,334 & 0,334 & 0,406 \\
\hline \multirow{6}{*}{0,10} & 0,10 & 0,14028 & 0,26131 & 0,35288 & 0,40952 & 0,42866 \\
\hline & 0,25 & 0,13860 & 0,25833 & 0,34902 & 0,40516 & 0,42412 \\
\hline & 0,50 & 0,13860 & 0,25834 & 0,34902 & 0,40516 & 0,42413 \\
\hline & 1,00 & $\begin{array}{l}0,13860 \\
\end{array}$ & 0,25833 & 0,34902 & 0,40516 & 0,42412 \\
\hline & 1,50 & 0,13860 & 0,25833 & 0,34902 & 0,40516 & 0,42412 \\
\hline & RIBEIRO[158] & 0,139 & 0,258 & 0,349 & 0,405 & 0,424 \\
\hline \multirow{6}{*}{0,20} & 0,10 & 0,160163 & 0,29613 & 0,39728 & 0,45937 & 0,48026 \\
\hline & 0,25 & 0,15976 & 0,29453 & 0,39519 & 0,45699 & 0,47779 \\
\hline & 0,50 & 0,15976 & 0,29453 & 0,39519 & 0,45698 & 0,47778 \\
\hline & 1,00 & 0,15976 & 0,29454 & 0,39520 & 0,45700 & 0,47779 \\
\hline & 1,50 & 0,15976 & 0,29454 & 0,39520 & 0,45700 & 0,47780 \\
\hline & RIBEIRO[158] & 0,160 & 0,295 & 0,395 & 0,457 & 0,478 \\
\hline \multirow{6}{*}{0,25} & 0,10 & 0,17618 & 0,32272 & 0,43117 & 0,49741 & 0,51966 \\
\hline & 0,25 & 0,17573 & 0,32169 & 0,42982 & 0,49586 & 0,51804 \\
\hline & 0,50 & 0,17562 & 0,32169 & 0,42981 & 0,49586 & 0,51803 \\
\hline & 1,00 & 0,17563 & 0,32170 & 0,42983 & 0,49587 & 0,51805 \\
\hline & 1,50 & 0,17563 & 0,32170 & 0,42983 & 0,49588 & 0,51805 \\
\hline & RIBEIRO[158] & 0,176 & 0,322 & 0,430 & 0,496 & 0,518 \\
\hline \multirow{6}{*}{0,30} & 0,10 & 0,19533 & 0,35547 & 0,47295 & 0,54429 & 0,56819 \\
\hline & 0,25 & 0,19502 & 0,35488 & 0,47217 & 0,54338 & 0,56724 \\
\hline & 0,50 & 0,19502 & 0,35488 & 0,47213 & 0,54338 & 0,56724 \\
\hline & 1,00 & 0,19503 & 0,35489 & 0,47216 & 0,54339 & 0,56725 \\
\hline & 1,50 & 0,19503 & 0,35489 & 0,47216 & 0,54340 & 0,56725 \\
\hline & RIBEIRO\{168] & 0,195 & 0,355 & 0,472 & 0,543 & 0,567 \\
\hline \multicolumn{2}{|c|}{ Teoria Clássica } & 0,132 & 0,246 & 0,334 & 0,388 & 0,406 \\
\hline
\end{tabular}


Acontece que para valores muito pequenos de " $\alpha$ ", devido a um problema de condicionamento numérico, os resultados não são tão bons quanto os da tabela 4.1, mesmo aumentando o número de pontos de integração. Neste trabalho a integração numérica é sempre obtida com 12 pontos de integração.

Este problema numérico pode ser contornado dividindo-se 0 elemento em sub-elementos, quando o ponto de colocação coincidir com um dos três nós do elemento. Cada sub-elemento terá um comprimento igual à distância que o separa do ponto fonte. Desta forma consegue-se resultados excelentes mesmo para valores de " $\alpha$ " iguais a 0,001 . Neste trabalho, quando não explicitado de forma diferente, considerou-se o valor de " $\alpha$ " igual a 0,5 , para os outros exemplos.

Na tabela 4.2 estão apresentados os valores do momento fletor " $M_{x \alpha}$ ", para a linha de centro da placa, $x_{2}=a / 2$. Nota-se que os valores dos momentos calculados pela Teoria de Reissner, via MEC, coincidem com os da teoria clássica quando $(\mathrm{h} / \mathrm{a})=0,01$.

Para os momentos, a influência da espessura não é tão significativa como no cálculo dos deslocamentos, ficando o acréscimo, para o centro da placa quando $(\mathrm{h} / \mathrm{a})=0,30$, inferior a $3 \%$.

Na tabela 4.3 estão apresentados os valores da flecha " $w$ " e do momento fletor " $M_{x x}$ " no centro da placa, além do momento de torção " $\mathrm{M}_{\mathrm{xy}}$ " no canto. Os valores calculados pelo MEC, usando-se a Teoria de Reissner são mais uma vez comparados com os valores analíticos fornecidos por RIBEIRO [158] e pela teoria clássica. Segundo RIBEIRO [140] o valor que conduz a melhor resultados é " $\alpha$ " $=0,25$, valor confirmado pelo exemplo 1, que mostrou também resultados semelhantes para " $\alpha$ " $=0,5$. 
Tabela 4.2 - Valores do momento fletor " $M_{x x}$ " sobre a linha de centro $\left(x_{2}=a / 2\right)$ da placa

\begin{tabular}{|c|c|c|c|c|c|c|}
\hline \multirow[t]{2}{*}{$\mathrm{h} / \mathrm{a}$} & \multirow[t]{2}{*}{$\alpha$} & \multicolumn{5}{|c|}{$M_{x x}\left(10 / q a^{2}\right)$} \\
\hline & & $0,10 \mathrm{~L}$ & $0,20 \mathrm{~L}$ & $0,30 \mathrm{~L}$ & $\overline{0,40 \mathrm{~L}}$ & $\overline{0,50 \mathrm{~L}}$ \\
\hline \multirow{6}{*}{0,01} & 0,10 & 0,21019 & 0,34392 & 0,42428 & 0,46642 & 0,47947 \\
\hline & 0,25 & 0,20914 & 0,34326 & 0,42365 & 0,46582 & 0,47888 \\
\hline & 0,50 & 0,20914 & 0,34326 & 0,42366 & 0,46582 & 0,47888 \\
\hline & 1,00 & 0,20913 & 0,34326 & 0,42366 & 0,46582 & 0,47888 \\
\hline & 1,50 & 0,21198 & 0,34307 & 0,42650 & 0,46877 & 0,48199 \\
\hline & RIBEIRO[158] & 0,209 & 0,343 & 0,424 & 0,466 & 0,479 \\
\hline \multirow{6}{*}{0,10} & 0,10 & 0,22212 & 0,34987 & 0,42960 & 0,47172 & 0,48481 \\
\hline & 0,25 & 0,20967 & 0,34417 & 0,42489 & 0,46724 & 0,48037 \\
\hline & 0,50 & 0,20962 & 0,34418 & 0,42489 & 0,46724 & 0,48037 \\
\hline & 1,00 & 0,20961 & 0,34417 & 0,42489 & 0,46724 & 0,48036 \\
\hline & 1,50 & 0,20962 & 0,34417 & 0,42489 & 0,46724 & 0,48036 \\
\hline & RIBEIRO[158] & 0,210 & 0,344 & 0,425 & 0,467 & 0,480 \\
\hline \multirow{6}{*}{0,20} & 0,10 & 0,22218 & 0,35092 & 0,43146 & 0,47414 & 0,48741 \\
\hline & 0,25 & 0,21114 & 0,34692 & 0,42859 & 0,47154 & 0,48485 \\
\hline & 0,50 & 0,21111 & 0,34690 & 0,42859 & 0,47153 & 0,48485 \\
\hline & 1,00 & 0,21110 & 0,34692 & 0,42860 & 0,47154 & 0,48486 \\
\hline & 1,50 & 0,21106 & 0,34692 & 0,42860 & 0,47154 & 0,48486 \\
\hline & RIBEIRO[158] & 0,211 & 0,347 & 0,429 & 0,472 & 0,485 \\
\hline \multirow{6}{*}{0,25} & 0,10 & 0,22216 & 0,35219 & 0,43348 & 0,47659 & 0,49000 \\
\hline & 0,25 & 0,21225 & 0,34899 & 0,43138 & 0,47476 & 0,48823 \\
\hline & 0,50 & 0,21223 & 0,34896 & 0,43137 & 0,47475 & 0,48822 \\
\hline & 1,00 & 0,21202 & 0,34898 & 0,43138 & 0,47477 & 0,48824 \\
\hline & 1,50 & 0,21218 & 0,34898 & 0,43139 & 0,47477 & 0,48824 \\
\hline & RIBEIRO[168] & 0,212 & 0,349 & 0,431 & 0,475 & 0,488 \\
\hline \multirow{6}{*}{0,30} & 0,10 & 0,22244 & 0,35406 & 0,43628 & 0,47992 & 0,49350 \\
\hline & 0,25 & 0,21360 & 0,35151 & 0,43478 & 0,47870 & 0,49235 \\
\hline & 0,50 & 0,21358 & 0,35148 & 0,43477 & 0,47870 & 0,49234 \\
\hline & 1,00 & 0,21355 & 0,35150 & 0,43479 & 0,47871 & 0,49236 \\
\hline & 1,50 & 0,21352 & 0,35150 & 0,43479 & 0,47872 & 0,49236 \\
\hline & RIBEIRO[168] & 0,214 & 0,352 & 0,435 & 0,479 & 0,492 \\
\hline \multicolumn{2}{|c|}{ Teoria Clássica } & 0,209 & 0,343 & 0,424 & 0,466 & 0,479 \\
\hline
\end{tabular}


Tabela 4.3 - Deslocamentos transversais " $w$ " e momento fletor " $M_{x \text { " }}$ no centro da placa; momento de torção " $M_{x y}$ " no canto.

\begin{tabular}{|c|c|c|c|c|c|c|}
\hline \multirow{2}{*}{$\mathrm{h} / \mathrm{a}$} & \multicolumn{2}{|c|}{$w_{x}\left(100 D / q a^{4}\right)$} & \multicolumn{2}{|c|}{$M_{x x}\left(10 / q a^{2}\right)$} & \multicolumn{2}{|c|}{$M_{x y}\left(10 / q a^{2}\right)$} \\
\hline & $\operatorname{MEC}(\alpha=0,5)$ & RIBEIRO[168] & $\operatorname{MEC}(\alpha=0,5)$ & RIBEIRO[158] & $\operatorname{MEC}(\alpha=0,5)$ & RIBEIRO[158] \\
\hline 0,010 & 0,40641 & 0,406 & 0,47888 & 0,479 & 0,33342 & 0,325 \\
\hline 0,025 & 0,40735 & 0,407 & 0,47896 & 0,479 & 0,32673 & 0,324 \\
\hline 0,050 & 0,41070 & 0,411 & 0,47924 & 0,479 & 0,32298 & 0,321 \\
\hline 0,075 & 0,41629 & 0,416 & 0,47971 & 0,480 & 0,31715 & 0,316 \\
\hline 0,100 & 0,42413 & 0,424 & 0,48037 & 0,480 & 0,31102 & 0,311 \\
\hline 0,125 & 0,43450 & 0,434 & 0,48148 & 0,481 & 0,30562 & 0,308 \\
\hline 0,150 & 0,44644 & 0,446 & 0,48219 & 0,482 & 0,30003 & 0,305 \\
\hline 0,175 & 0,46101 & 0,461 & 0,48346 & 0,483 & 0,29856 & 0,302 \\
\hline 0,200 & 0,47778 & 0,478 & 0,48485 & 0,485 & 0,28978 & 0,297 \\
\hline 0,250 & 0,51803 & 0,518 & 0,48822 & 0,488 & 0,27537 & 0,287 \\
\hline 0,300 & 0,56724 & 0,567 & 0,49234 & 0,492 & 0,25426 & 0,274 \\
\hline T. Clássica & \multicolumn{2}{|c|}{0,406} & \multicolumn{2}{|c|}{0,479} & \multicolumn{2}{|c|}{0,325} \\
\hline
\end{tabular}

Deste exemplo pode-se concluir que a colocação do ponto fora do domínio conduz a resultados semelhantes aos obtidos analiticamente, desde que se escolha valores adequados de " $\alpha$ ". A colocação do ponto fora do domínio conduz a simplificaçōes, evitando-se as integrações analíticas para o elemento que contém o nó singular. No entanto esta técnica conduz a alguns resultados ruins, como por exemplo a reação de apoio próxima aos cantos, mesmo para " $\alpha$ " pequeno e com muitos pontos de integração.

Como melhoramento desta técnica pode-se dividir o elemento em sub-elementos, obtendo-se desta forma bons resultados, mesmo para as reações no contorno da placa. As figuras $4.11,4.12$ e 4.13 mostram as 
reações no contorno considerando-se as condiçōes "HARD" e "SOFT", para três relações (h/a) distintas e os resultados são comparados com os obtidos por BATHE [159]. Nota-se que os valores são praticamente coincidentes para as duas primeiras relaçōes, " $h / a=0,1$ " e " $h / a=0,01$ ", ficando os resultados com valores dispersos próximo ao canto, para a placa fina representada pela Fig. 4.12, cuja relação " $\mathrm{h} / \mathrm{a}=0,001^{\text {n }}$. Foi adotado o valor " $\alpha=0,01$ " para as três relações analisadas.
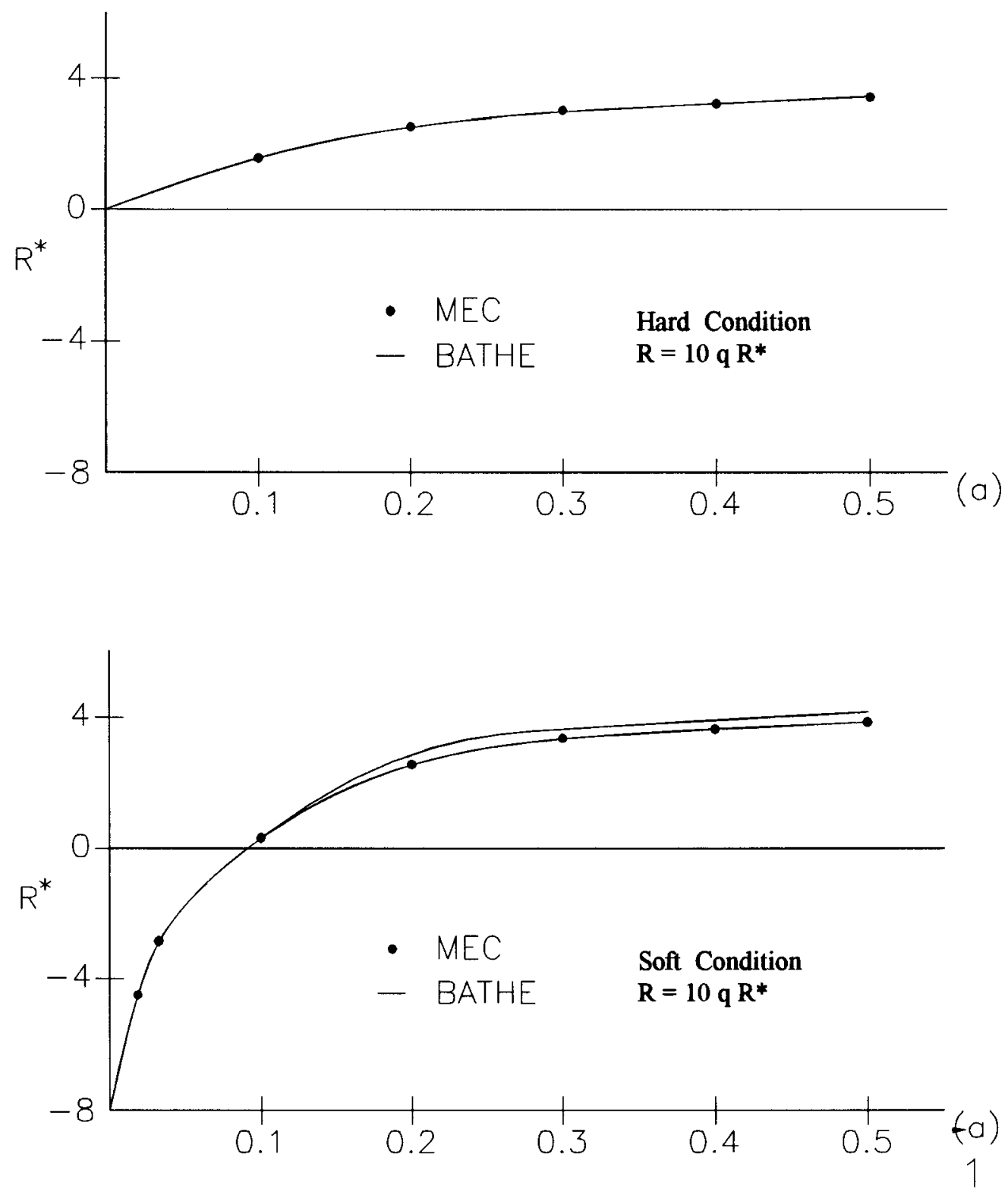

Fig. 4.11 -Reações no contorno para " $h / a=0,1$ ". 

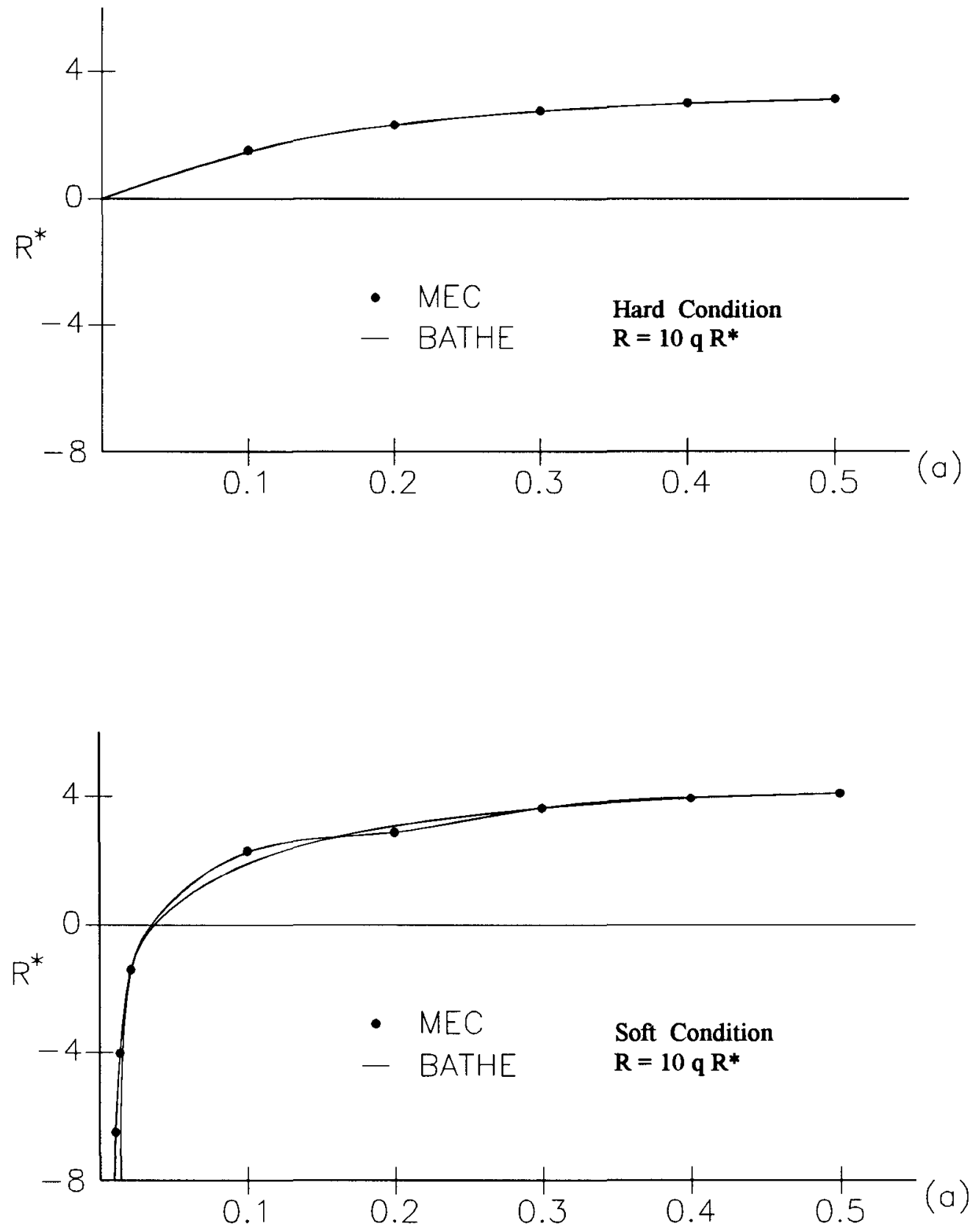

Fig. 4.12 -Reações no contorno para " $h / a=0,01$ ". 

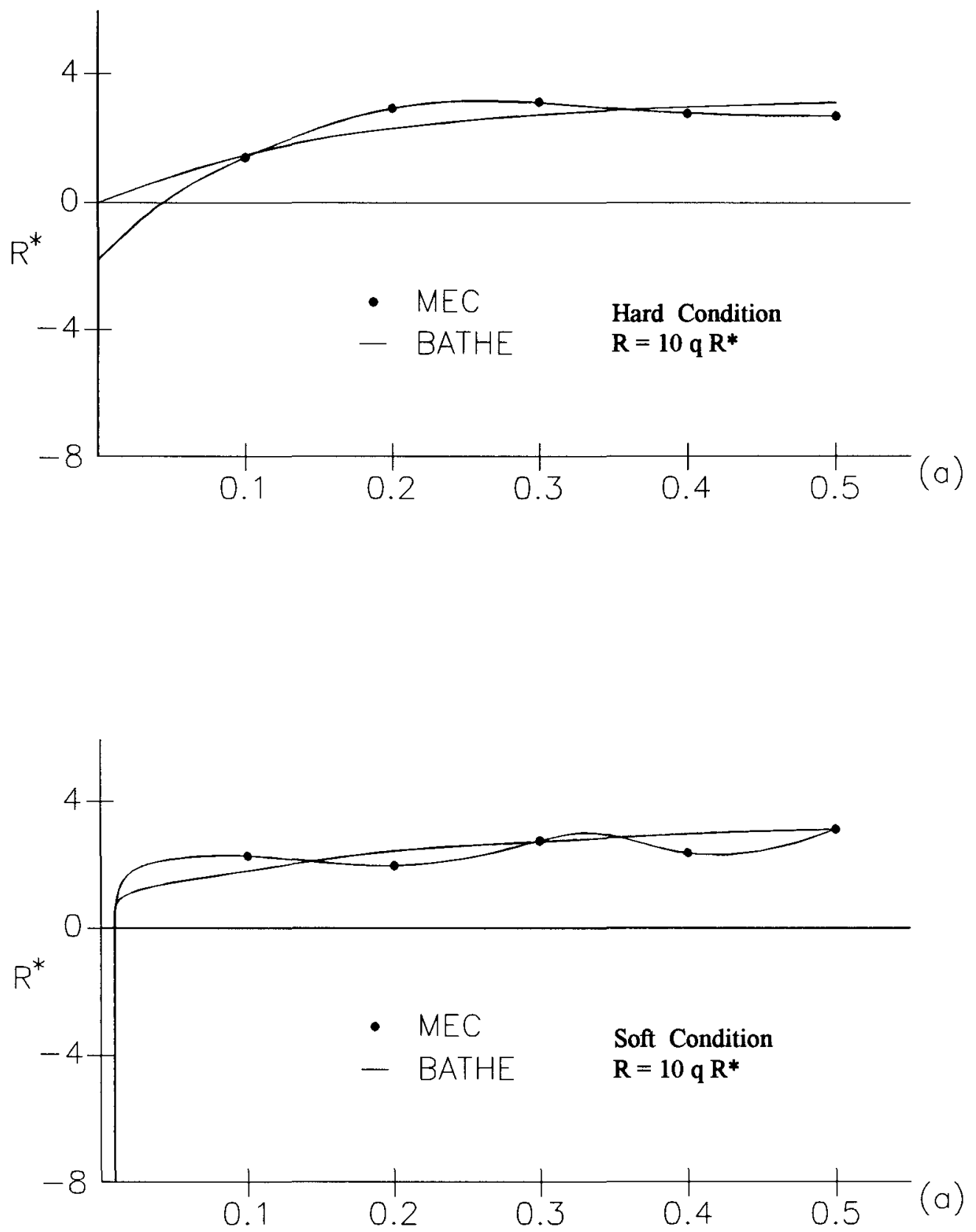

Fig. 4.13 -Reações no contorno para " $h / a=0,001 "$. 


\subsection{2 - EXEMPLO 2: Placa alongada.}

Na Fig. 4.14 está representada uma placa alongada com $1 \mathrm{~m}$ de largura e $8 \mathrm{~m}$ de comprimento, tendo um dos lados menores engastados e os outros livres. São colocados 2 apoios pontuais a $2 \mathrm{~m}$ da extremidade menor livre, onde é aplicada uma carga de $1000 \mathrm{kgf}$. É mostrado logo abaixo nesta mesma figura, o esquema da viga análogo à placa analisada.

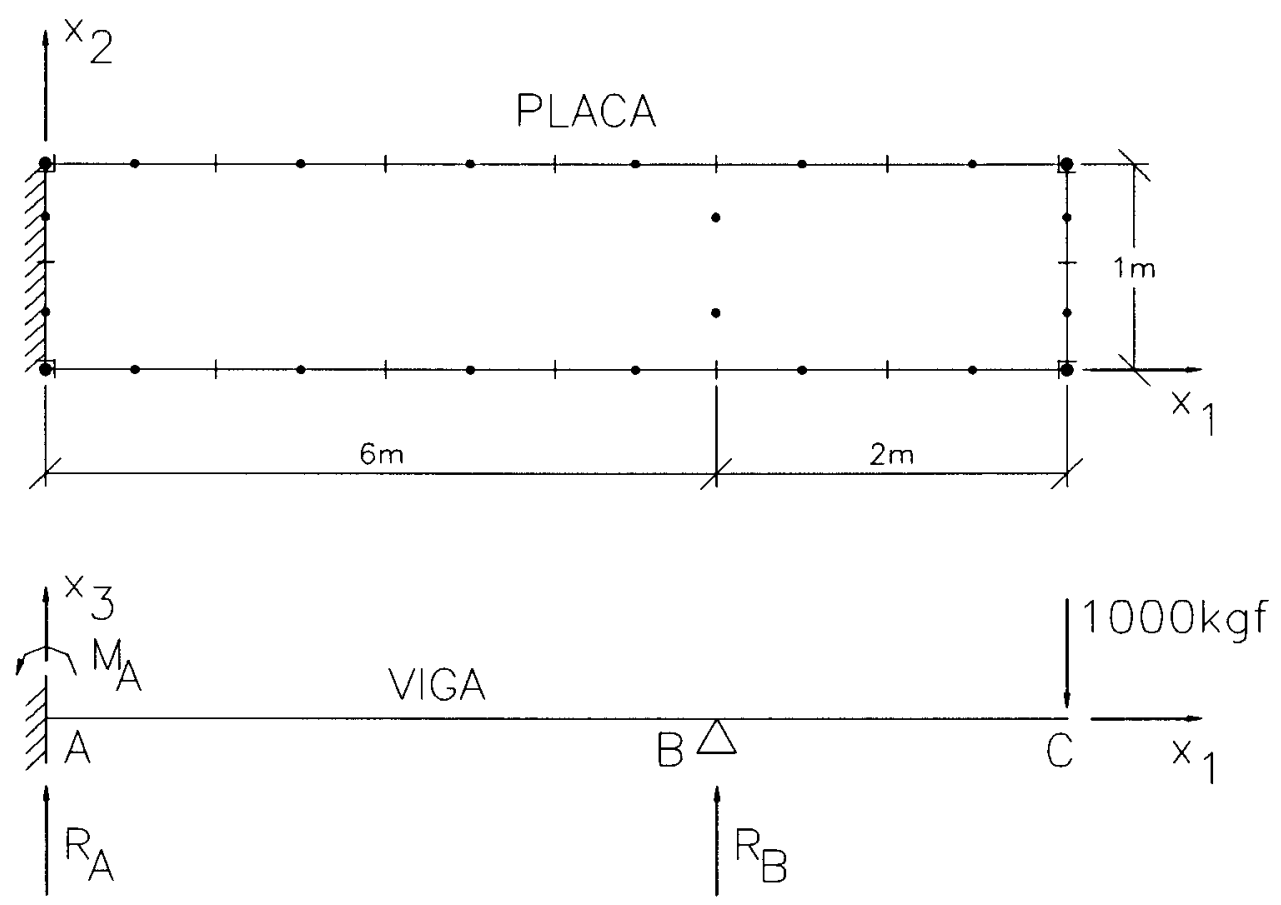

Fig. 4.14 -Placa alongada.

A espessura da placa é de $15 \mathrm{~cm}$, ○ módulo de elasticidade $E=21.000 \mathrm{MPa}$, o coeficiente de Poisson $v=0,25$ e a constante $\alpha=0,25$.

Na tabela 4.4, estão mostrados os resultados da placa analisada e os correspondentes da viga, para as reações nos pontos $\mathrm{A}$ e $\mathrm{B}$, o momento fletor no ponto $\mathrm{B}$ e a flecha no ponto $\mathrm{C}$. Os resultados desta tabela confirmam, como esperado, que o cálculo da placa analisada com a forma alongada se assemelha ao de uma viga. 
Tabela 4.4 - Resultados do Exemplo 2

\begin{tabular}{|c|c|c|}
\hline VALORES & VIGA & PLACA \\
\hline$R_{A}$ (kgf) & -500 & $-500,9$ \\
\hline$M_{\mathbf{A}}$ (kgf.m) & -1000 & $-1005,3$ \\
\hline$R_{\mathbf{B}}$ (kgf) & +1500 & $+1500,8$ \\
\hline$M_{\mathbf{x}, \mathbf{B}}$ (kgf.m) & -2000 & $-2109,1$ \\
\hline$w_{C}$ (cm) & $-1,51$ & $-1,47$ \\
\hline
\end{tabular}

\subsection{3 - EXEMPLO 3: Placa quadrada com apoios internos.}

Será analisado neste exemplo uma placa quadrada submetida a um carregamento uniformemente distribuído em toda a área, apoiada apenas em quatro apoios internos. Os resultados serão comparados com os obtidos pelo programa "SUPERSAP - Versão 7.52".

A placa foi discretizada em 4 e 24 elementos de contorno para análise pelo MEC e em 9 e 225 elementos finitos para análise via "SUPERSAP". As discretizaçōes em elementos de contorno e em elementos finitos estão mostradas na Fig. 4.15.

a)

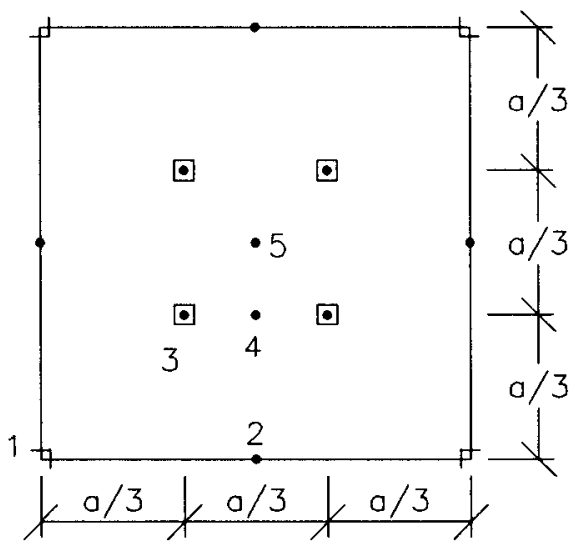

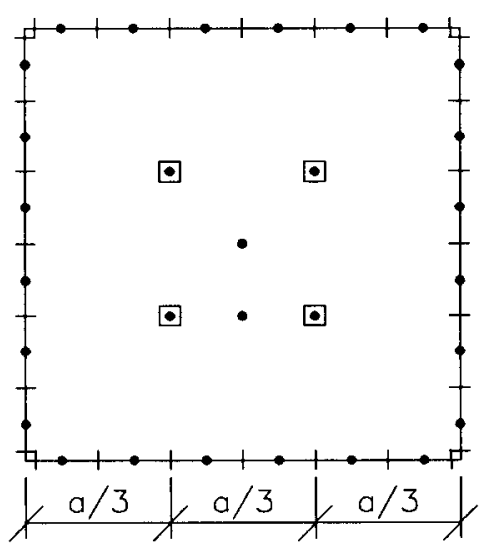


b)
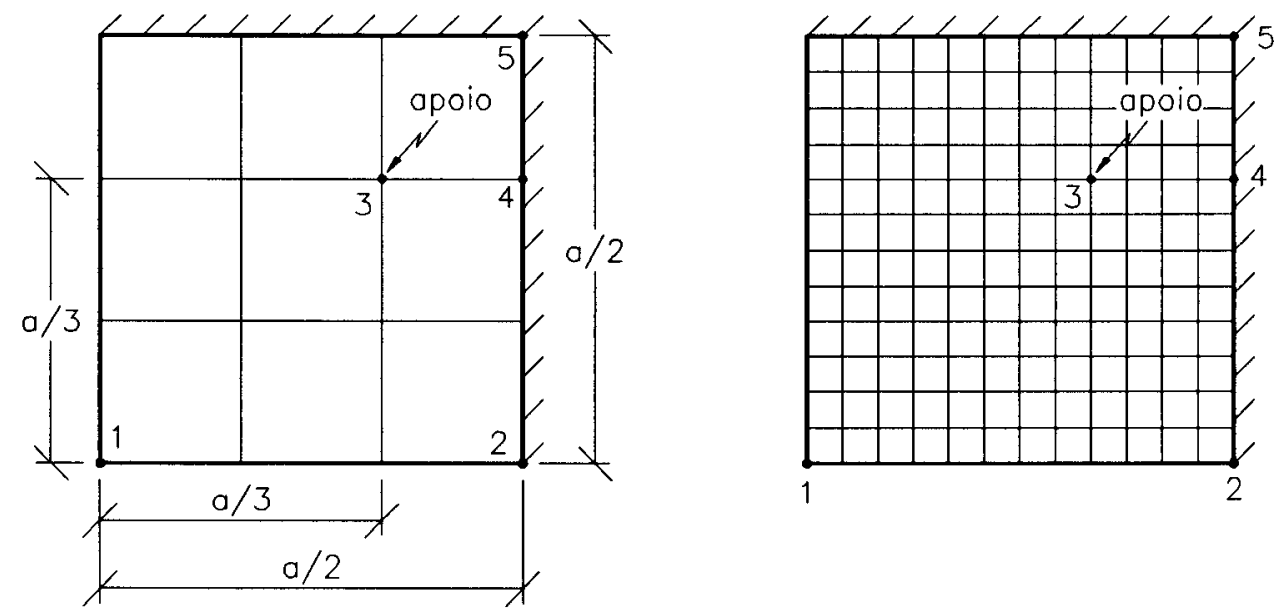

Fig. 4.15 -a) Discretização em 4 e 24 elementos de contorno.

b) Discretização em 9 e 225 elementos finitos (1/4 da placa).

O vão "a" é de $300 \mathrm{~cm}$, a placa tem $10 \mathrm{~cm}$ de espessura e a carga uniformemente distribuída é de $-5 \mathrm{kN} / \mathrm{m}^{2}$. O módulo de elasticidade longitudinal do material $E=25.000 \mathrm{MPa}$ e o coeficiente de Poisson $v=0,1667$.

Os resultados dos deslocamentos e momentos fletores nos pontos mostrados na Fig. 4.15 estão apresentados respectivamente nas tabelas 4.5 e 4.6.

Tabela 4.5 - Deslocamento transversal "w" (cm)

\begin{tabular}{|c|c|c|c|c|c|c|}
\hline \multirow{2}{*}{ Ponto } & \multicolumn{2}{|c|}{ MEC - 4 el. cont. } & \multicolumn{2}{c|}{ MEC - 24 el. cont. } & \multicolumn{2}{c|}{ SUPERSAP } \\
\cline { 2 - 7 } & $\begin{array}{c}4 \text { apoios } \\
\text { pontuais }\end{array}$ & $\begin{array}{c}\text { 4 apoios } \\
\text { em área }\end{array}$ & $\begin{array}{c}4 \text { apoios } \\
\text { pontuais }\end{array}$ & $\begin{array}{c}4 \text { apoios } \\
\text { em área }\end{array}$ & $\begin{array}{c}\mathbf{9} \\
\text { elementos }\end{array}$ & $\begin{array}{c}\mathbf{2 2 5} \\
\text { elementos }\end{array}$ \\
\hline 1 & $-0,1519$ & $-0,1504$ & $-0,1348$ & $-0,1334$ & $-0,1417$ & $-0,1354$ \\
\hline 2 & $-0,0613$ & $-0,0602$ & $-0,0655$ & $-0,0645$ & $-0,0672$ & $-0,0646$ \\
\hline 4 & $+0,0089$ & $+0,0095$ & $+0,0092$ & $+0,0098$ & $+0,0107$ & $+0,0098$ \\
\hline 5 & $+0,0202$ & $+0,0207$ & $+0,0209$ & $+0,0214$ & $+0,0232$ & $+0,0215$ \\
\hline
\end{tabular}


Tabela 4.6 - Momentos fletores $(\mathrm{kNxcm})$

\begin{tabular}{|c|c|c|c|c|c|c|c|c|c|}
\hline \multirow[t]{2}{*}{ PONTO } & & \multicolumn{2}{|c|}{$\begin{array}{c}\text { MEC } \\
4 \text { elementos }\end{array}$} & \multicolumn{2}{|c|}{$\begin{array}{c}\text { MEC } \\
24 \text { elementos }\end{array}$} & \multicolumn{2}{|c|}{$\begin{array}{l}\text { SUPERSAP } \\
9 \text { elementos }\end{array}$} & \multicolumn{2}{|c|}{$\begin{array}{c}\text { SUPERSAP } \\
225 \text { el. } \\
\end{array}$} \\
\hline & & $M_{1}$ & $M_{2}$ & $M_{1}$ & $\mathrm{M}_{2}$ & $M_{1}$ & $M_{2}$ & $M_{1}$ & $M_{2}$ \\
\hline \multirow[t]{2}{*}{3} & $\begin{array}{l}4 \text { apolos } \\
\text { pontuais }\end{array}$ & $\$ 11,42$ & $-611,42$ & $\$ 17,64$ & $-617,64$ & \multirow{2}{*}{$-204,40$} & \multirow{2}{*}{$-2004,40$} & \multirow{2}{*}{$-347,67$} & \multirow[t]{2}{*}{$-347,67$} \\
\hline & $\begin{array}{l}4 \text { apolos } \\
\text { em área }\end{array}$ & $-341,24$ & 341,24 & 348,13 & $-348,13$ & & & & \\
\hline \multirow{2}{*}{4} & $\begin{array}{l}\text { A apolos } \\
\text { pontuais }\end{array}$ & $-177,85$ & $-234,49$ & $-184,15$ & $-243,19$ & \multirow{2}{*}{$-215,08$} & \multirow{2}{*}{$-237,00$} & \multirow{2}{*}{$-187,00$} & \multirow{2}{*}{$-242,58$} \\
\hline & $\begin{array}{l}4 \text { apoios } \\
\text { em irea }\end{array}$ & $-179,24$ & -232,37 & $-185,52$ & $-241,04$ & & & & \\
\hline \multirow[t]{2}{*}{6} & $\begin{array}{l}4 \text { apolos } \\
\text { pontuais }\end{array}$ & $-211,23$ & $-211,23$ & $-218,48$ & $-218,48$ & \multirow{2}{*}{$-248,83$} & \multirow{2}{*}{$-248,83$} & \multirow{2}{*}{$-218,50$} & \multirow{2}{*}{$-218,50$} \\
\hline & $\begin{array}{l}\text { A apolos } \\
\text { em area }\end{array}$ & $-210,89$ & $-210,89$ & $-218,11$ & $-218,11$ & & & & \\
\hline
\end{tabular}

Considerando-se a solução em elementos de contorno, foram considerados 4 apoios pontuais ou 4 apoios distribuidos numa área de $20 \times 20 \mathrm{~cm}^{2}$. Analisando-se a tabela 4.5 nota-se que os valores dos deslocamentos são bons, mesmo para uma discretização pobre, tanto em elementos de contorno como em elementos finitos. No cálculo dos momentos fletores foi considerado a média dos quatro elementos finitos concorrentes no nó analisado. Os resultados obtidos com os elementos de contorno mostraram-se bastante satisfatórios, mesmo para uma discretização pobre de um elemento de contorno por lado da placa.

A discrepância observada no momento fletor do ponto 3 para o caso dos apoios pontuais se explica devido à descontinuidade deste esforço para esta situação, pois sabe-se que o momento fletor tende para infinito nos pontos correspondentes a estes apoios. O valor tabelado neste caso representa o momento fletor para um ponto situado a uma distância muito pequena do apoio, neste caso igual a $1 \mathrm{~cm}$. 


\subsection{4 - EXEMPLO 4: Placa com 6 apoios internos}

Neste exemplo analisa-se uma placa retangular de $12 \times 8 \mathrm{~m}^{2}$ apoiada em 6 apoios internos, conforme Fig. 4.16.

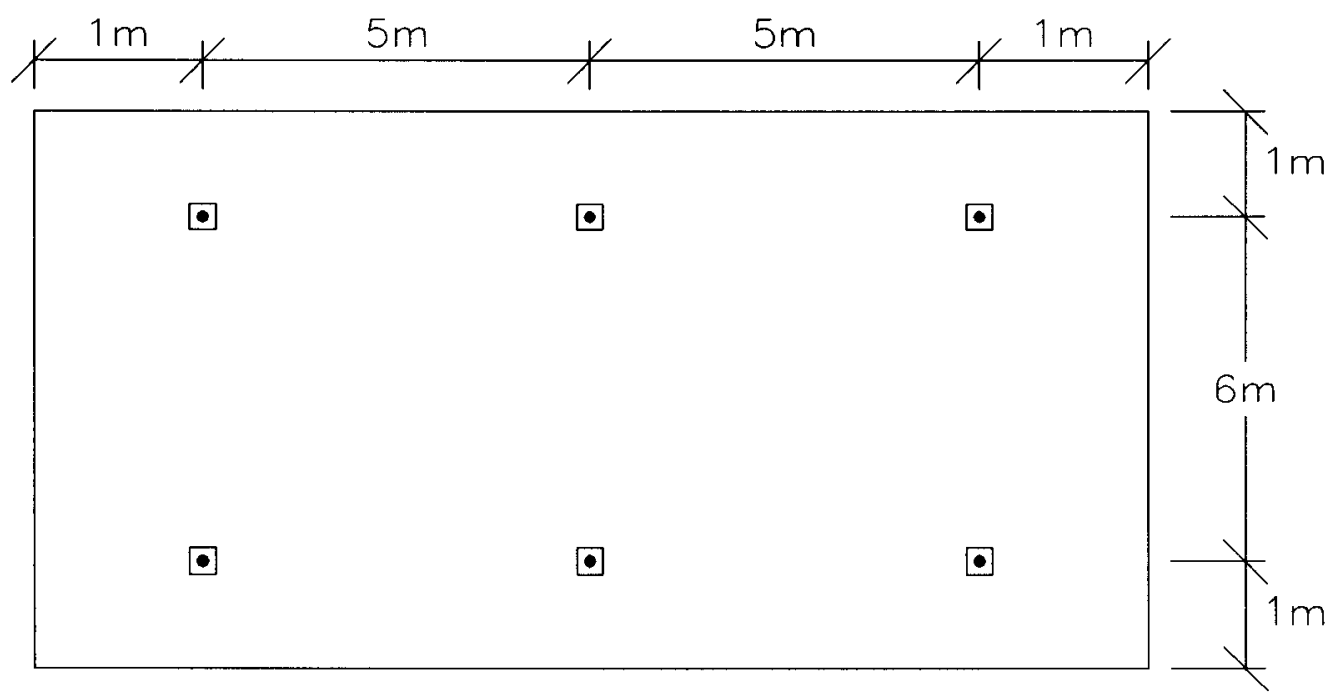

Fig. 4.16 - Placa do Exemplo 3.

A placa tem uma espessura de $15 \mathrm{~cm}$ e está submetida a um carregamento uniformemente distribuído de $-8 \mathrm{kN} / \mathrm{m} 2$, o coeficiente de Poisson é igual a 0,2 e o módulo de elasticidade longitudinal é igual a 25.000 MPa. As discretizaçōes em elementos de contorno e em elementos finitos estão mostradas na Fig. 4.17. 
a)

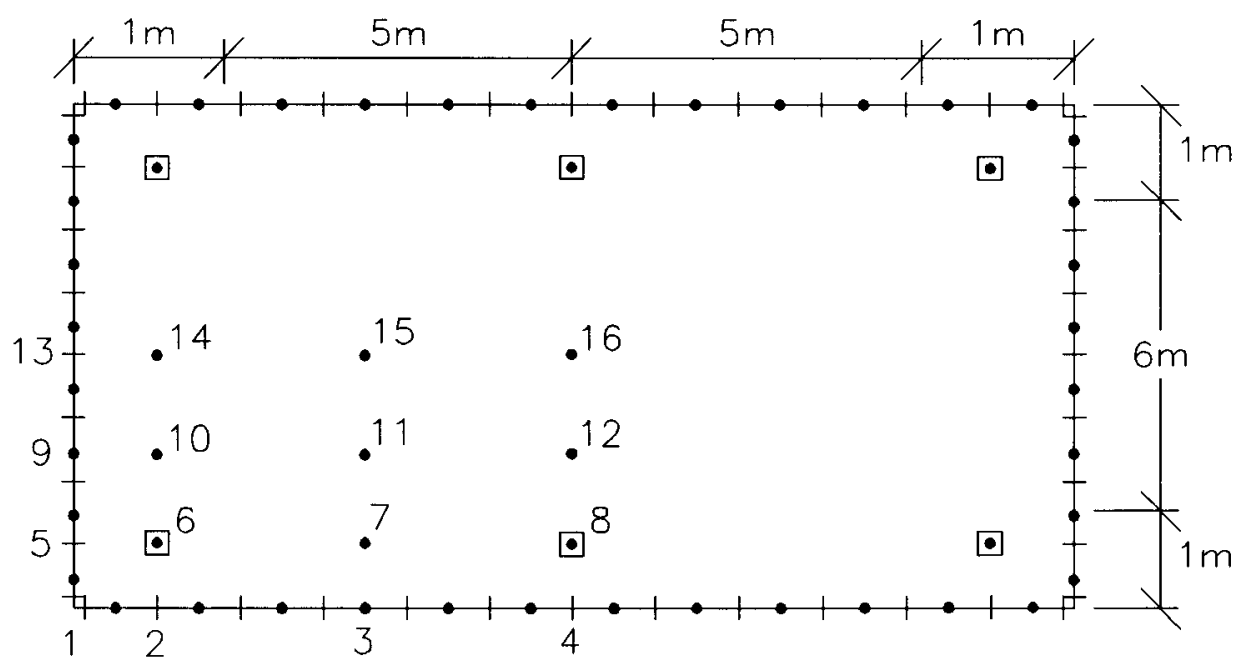

b)

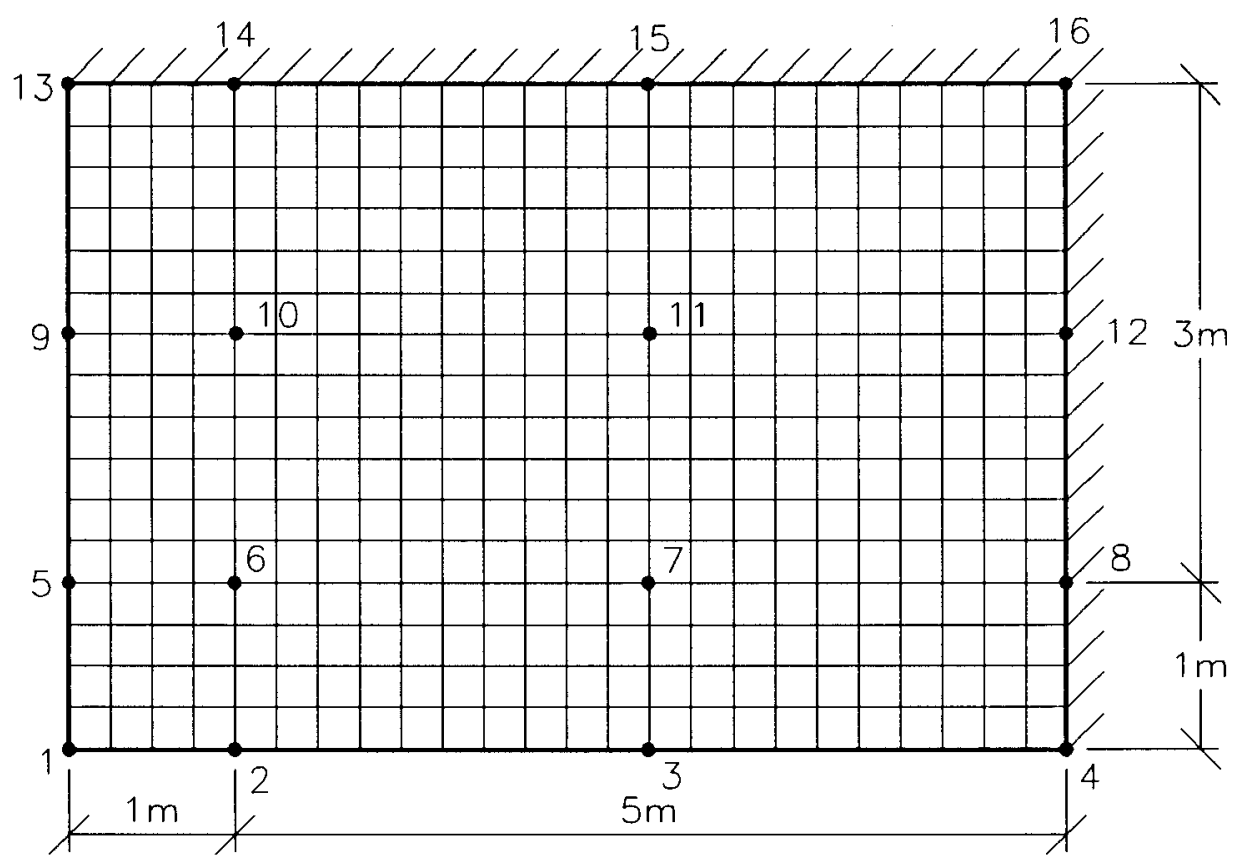

Fig. 4.17 -a) Discretização em elementos de contorno b) Discretização em elementos finitos

Os deslocamentos verticais e os momentos fletores nos nós indicados na Fig. 4.17 estão relacionados nas tabelas 4.7 e 4.8 respectivamente. 
Tabela 4.7 - Deslocamentos verticais para o Exemplo 4.

\begin{tabular}{|c|c|c|}
\hline \multirow{2}{*}{ PONTO } & \multicolumn{2}{|c|}{$w(\mathrm{~cm})$} \\
\hline & MEC & SUPERSAP \\
\cline { 2 - 3 } & $+1,039$ & $+1,015$ \\
\hline 2 & $+0,789$ & $+0,789$ \\
\hline 3 & $+0,340$ & $+0,344$ \\
\hline 4 & $+0,795$ & $+0,797$ \\
\hline 5 & $+0,180$ & $+0,180$ \\
\hline 7 & $-0,429$ & $-0,424$ \\
\hline 9 & $-1,056$ & $-1,044$ \\
\hline 10 & $-1,184$ & $-1,176$ \\
\hline 11 & $-1,451$ & $-1,444$ \\
\hline 12 & $-1,272$ & $-1,266$ \\
\hline 13 & $-1,581$ & $-1,568$ \\
\hline 14 & $-1,672$ & $-1,662$ \\
\hline 15 & $-1,876$ & $-1,868$ \\
\hline 16 & $-1,789$ & $-1,782$ \\
\hline
\end{tabular}

Tabela 4.8 - Momentos fletores para o Exemplo 4.

\begin{tabular}{|c|c|c|c|c|}
\hline \multirow{3}{*}{ PONTO } & \multicolumn{4}{|c|}{$M\left(\mathrm{kN} / \mathrm{cm}^{2}\right)$} \\
\cline { 2 - 5 } & \multicolumn{3}{|c|}{$\mathrm{MEC}$} & $\mathrm{M}_{2}$ \\
\cline { 2 - 5 } & $\mathrm{M}_{1}$ & $\mathrm{M}_{2}$ & $\mathrm{M}_{1}$ & -1279 \\
\hline 6 & -1892 & -1644 & -1494 & +591 \\
\hline 7 & +1575 & +587 & +1560 & -2556 \\
\hline 8 & -4877 & -3338 & -4035 & +2358 \\
\hline 10 & +216 & +2371 & +224 & +2193 \\
\hline 11 & +1275 & +2198 & +1272 & -2452 \\
\hline 12 & -487 & +2474 & -489 & +3268 \\
\hline 14 & +465 & +3287 & +477 & +3024 \\
\hline 15 & +1141 & +3032 & +1141 & +3383 \\
\hline 16 & +219 & +3396 & +216 & \\
\hline
\end{tabular}

Analisando-se os valores das tabelas 4.7 e 4.8 nota-se que os deslocamentos são praticamente coincidentes, as diferenças apresentadas nos momentos fletores se referem apenas aos apoios. 


\subsection{5 - EXEMPLO 5: Placa com uma viga interna}

Analisa-se neste exemplo uma placa continuamente apoiada no contorno cujas dimensões e propriedades são as mesmas do exemplo 3. A vinculação interna é produzida por uma barra, conforme mostrado na Fig. 4.18, onde estão indicadas as discretizações do contorno e da barra interna.

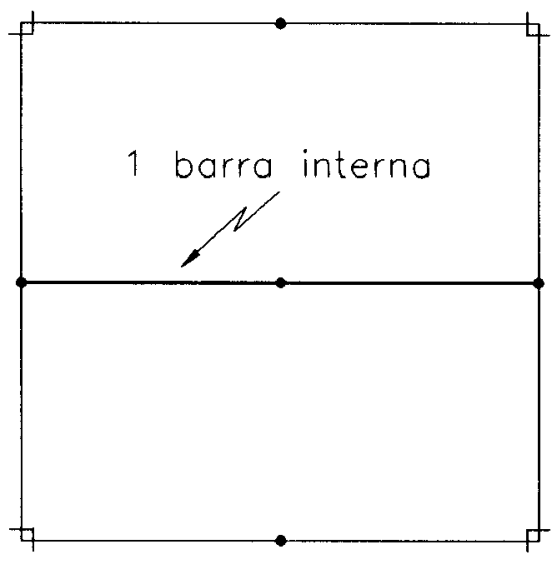

a)

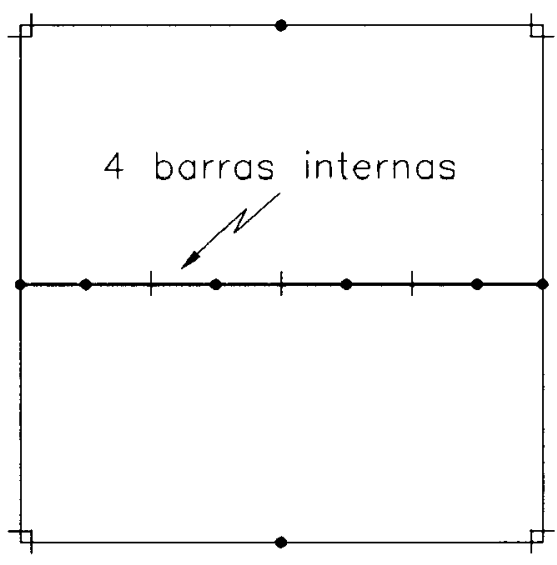

b)

Fig. 4.18 -Discretização para o Exemplo 5

a) Placa com uma barra interna

b) Placa com quatro barras internas

A barra interna tem seção de $20 \times 40 \mathrm{~cm}^{2}$ e foram consideradas as rigidezes geométricas à flexão e à torção, sem nenhuma redução.

Tabela 4.9 - Deslocamento w no centro da placa

\begin{tabular}{|c|c|c|c|c|}
\hline \multirow{2}{*}{$\begin{array}{c}\text { Espessura } \\
(\mathrm{cm})\end{array}$} & \multicolumn{4}{|c|}{$\mathrm{w}(\mathrm{cm})$} \\
\cline { 2 - 4 } & \multicolumn{3}{|c|}{ MEC } & ANSYS \\
& Placa & Placa com 1 & Placa com 4 & Versão \\
& bem & barra & barras & 5.0 \\
& enrijecimento & interna & internas & \\
\hline 10 & $7,712 \times 10^{-2}$ & $2,736 \times 10^{-2}$ & $2,432 \times 10^{-2}$ & $2,425 \times 10^{-2}$ \\
\hline 30 & $2,957 \times 10^{-3}$ & $2,811 \times 10^{-3}$ & $2,652 \times 10^{-3}$ & $2,611 \times 10^{-3}$ \\
\hline
\end{tabular}


O valor do deslocamento transversal para o centro da placa está apresentado na Tabela 4.9. Foram consideradas placas com 10 e $30 \mathrm{~cm}$ de espessura. Na primeira coluna apresentam-se os resultados para a placa sem enrijecimento, conforme o exemplo 1. Na segunda e terceira colunas estão representados respectivamente os valores dos deslocamentos para as discretizações internas a) e b) da Fig. 4.18. Na última coluna aparecem os resultados obtidos pelo programa ANSYS, versão 5.0, utilizando-se 100 elementos de placa SHELL63 e 10 elementos de barra BEAM4. Analisandose a tabela 4.9, nota-se um bom nível de enrijecimento introduzido pela barra no primeiro caso e evidentemente no segundo, com h=30 cm, o mesmo praticamente não foi sentido, devido à grande rigidez da placa à flexão.

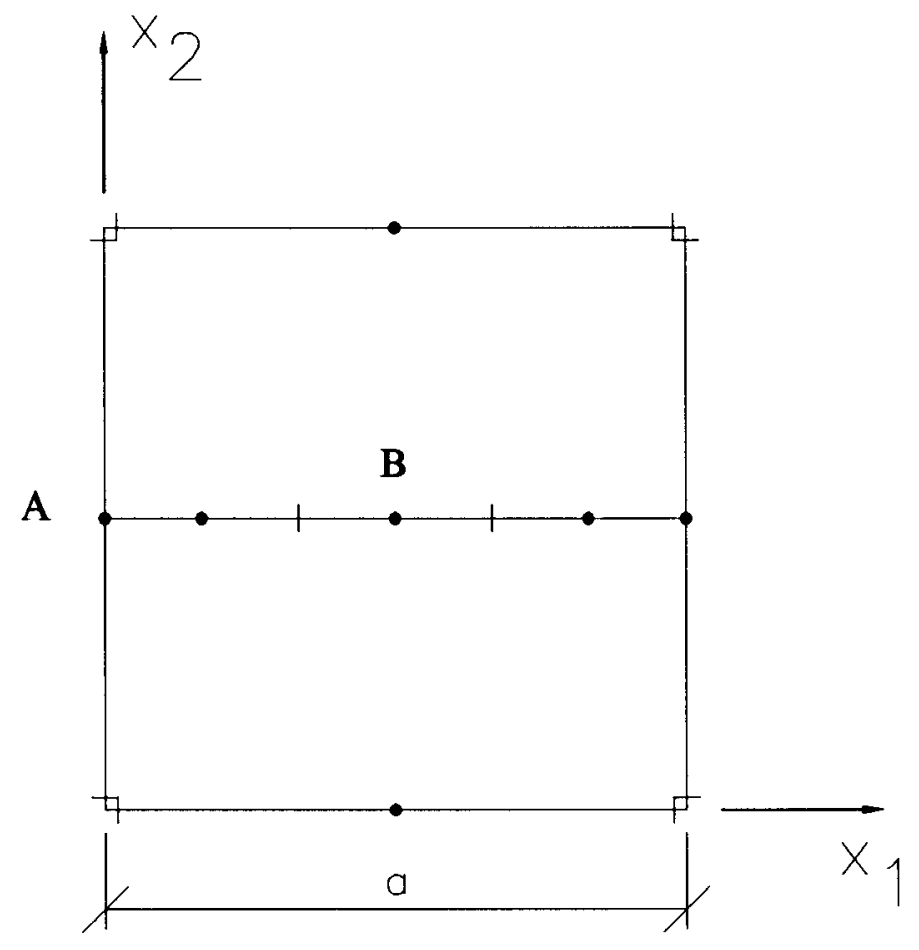

Fig. 4.19 - Discretização para cálculo dos momentos.

Os momentos fletores na viga são apresentados na Tabela 4.10, para os pontos $A$ e $B$, conforme Fig. 4.19. Nesta figura, a viga interna está 
dividida em 3 barras e a espessura da placa é de $10 \mathrm{~cm}$. Os resultados são comparados com os obtidos por CAMPOS [160] através do programa PLENOR. O valor do momento fletor no ponto $A$ se deve à diferença de curvaturas entre a placa e a viga.

Tabela 4.10 - Momentos fletores na viga (kgf.m)

\begin{tabular}{|c|c|c|}
\hline & MEC & PLENOR \\
\hline$M_{A}$ & 18,4 & 0,2 \\
\hline$M_{B}$ & 704,8 & 693,5 \\
\hline
\end{tabular}

4.5.6 - EXEMPLO 6: Placa quadrada apoiada em vigas elásticas.

Normalmente as lajes são calculadas considerando-se as vigas do contorno com rigidez infinita, ou seja, indeformáveis na direção vertical, conforme o exemplo da placa quadrada analisada no exemplo 1.

A associação da placa com as vigas flexiveis do contorno só tem solução exata conhecida para alguns casos particulares, o que torna tal análise possível apenas com a utilização de processos numéricos. $\mathrm{Na}$ Fig. 4.20 é apresentada uma placa quadrada com 4 vigas no contorno e 4 apoios rígidos nos cantos. A placa está submetida a um carregamento uniformemente distribuido " $q$ " e foi discretizada em 16 elementos de contorno. Cada viga do contorno está dividida em 4 barras correspondentes à discretização anterior.

As vigas tem uma rigidez a flexão $E I=5$ a $D ; 0$ coeficiente de Poisson $v=0,3$ e a constante $\alpha=0,25$ 


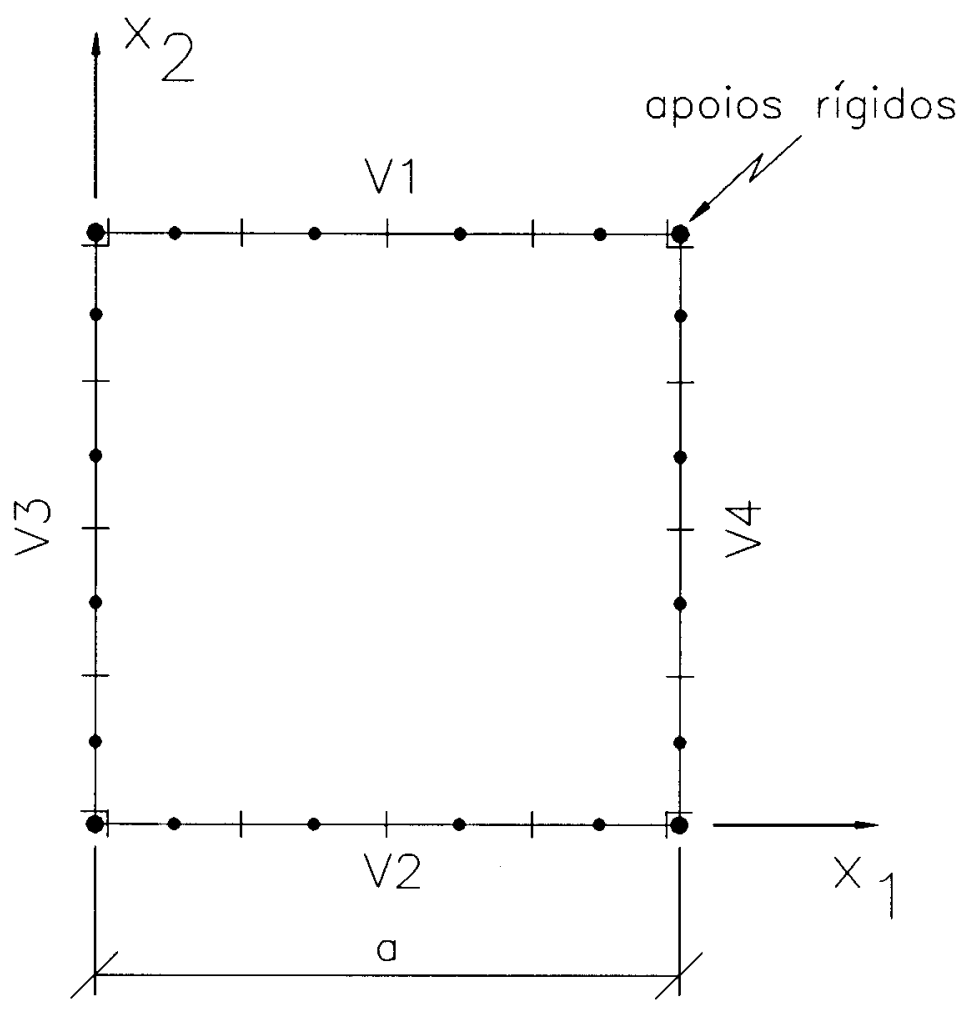

Fig. 4.20 - Placa apoiada em vigas elásticas.

Na Tabela 4.11 estão apresentados os resultados do deslocamento e momentos fletores no centro da placa. A primeira coluna corresponde aos valores obtidos neste trabalho $\mathrm{e}$ as demais foram apresentadas por PAIVA [113], utilizando a Teoria de Kirchhoff para uma placa discretizada em 24 elementos de contorno.

Tabela 4.11 - Resultados do Exemplo 6

\begin{tabular}{|c|c|c|c|}
\hline & $\begin{array}{c}\text { MEC } \\
\text { Reissner }\end{array}$ & $\begin{array}{c}\text { MEC } \\
\text { Kirchhoff [113] }\end{array}$ & $\begin{array}{c}\text { Solução } \\
\text { Exata [113] }\end{array}$ \\
\hline$(w 100 \mathrm{D}) /\left(q \mathrm{a}^{4}\right)$ & 0,484 & 0,560 & 0,519 \\
\hline$\left(\mathrm{M}_{\mathrm{x}} 10\right) /\left(q \mathrm{a}^{2}\right)$ & 0,458 & 0,534 & 0,494 \\
\hline$\left(\mathrm{M}_{\mathrm{y}} 10\right) /\left(\mathrm{q} \mathrm{a}^{2}\right)$ & 0,458 & 0,534 & 0,494 \\
\hline
\end{tabular}


4.5.7 - EXEMPLO 7: Placa com duas vigas internas cruzadas

A placa deste exemplo está mostrada na Fig. 4.21 e apresenta as mesmas propriedades do exemplo 5 , com carga transversal $q=-5 \mathrm{kN} / \mathrm{m}^{2}$.

Os resultados para a flecha máxima e os momentos nas vigas estão apresentados na tabela 4.12 .

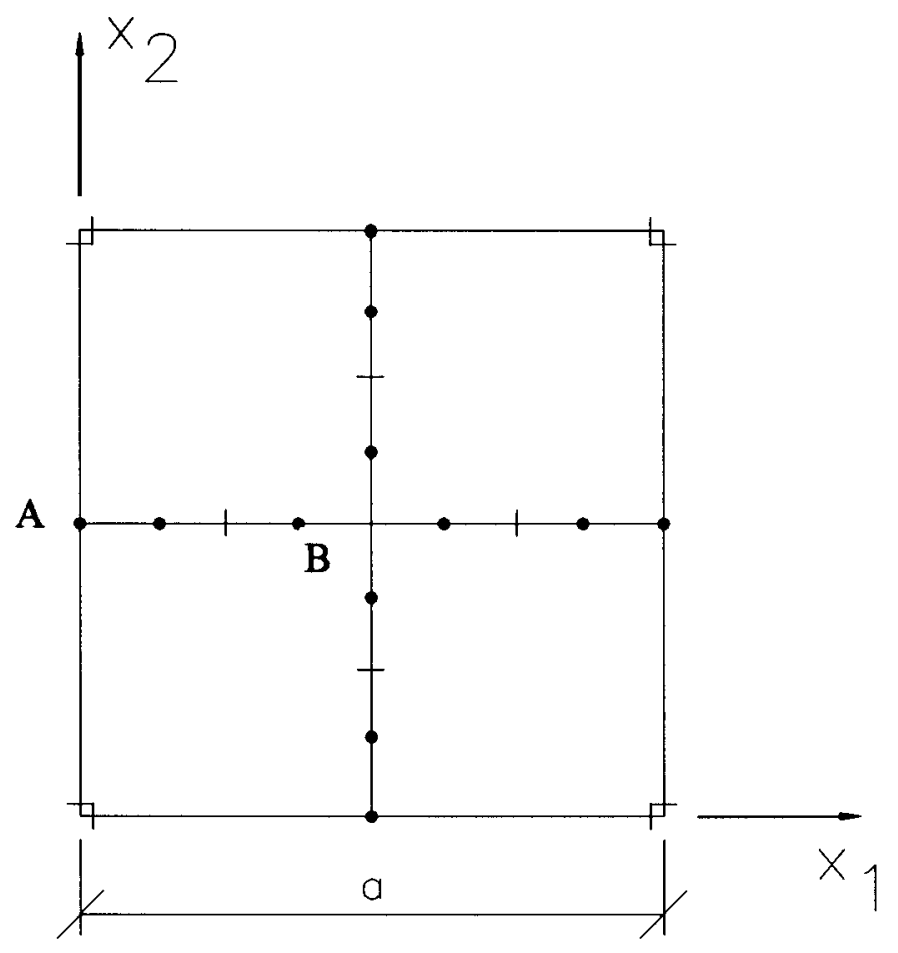

Fig. 4.21 - Placa do Exemplo 7.

Tabela 4.12 - Flecha e momentos da viga para o Exemplo 7.

\begin{tabular}{|c|c|c|}
\hline & MEC & PLENOR [160] \\
\hline$W_{B}$ (cm) & 0,016 & 0,015 \\
\hline$M_{A}$ (kgf.m) & 13,2 & 0,2 \\
\hline$M_{B}$ (kgf.m) & 403,6 & 395,5 \\
\hline
\end{tabular}


4.5.8 - EXEMPLO 8: Placa com duas e quatro vigas internas.

As duas placas analisadas estão apresentadas na Fig. 4.22, mostrando a discretização do contorno e das vigas internas. A placa é quadrada e tem dimensão $a=120 \mathrm{~cm}$, espessura $h=1 \mathrm{~cm}$, módulo de elasticidade $E=210.000 \mathrm{MPa}, q=-0,5 \mathrm{kN} / \mathrm{m}^{2}, v=0,33$ e as vigas têm dimensão de $0,5 \times 5 \mathrm{~cm}^{2}$.

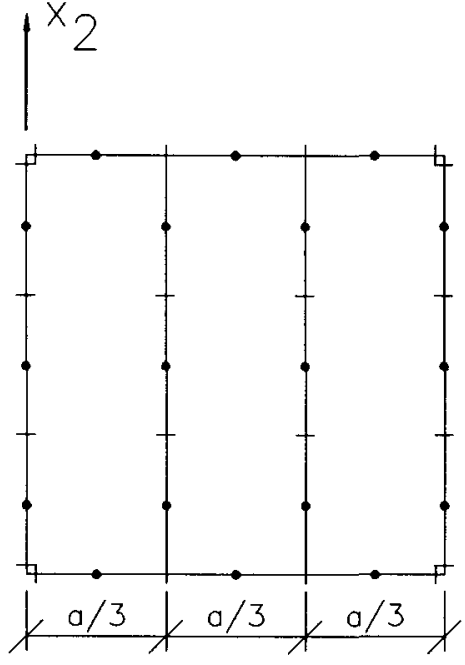

a)

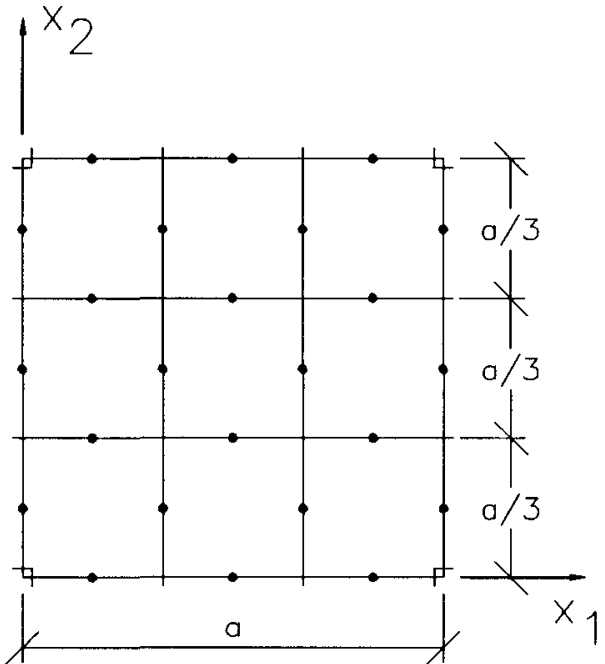

b)

Fig. 4.22 -a) Placa com duas barras internas paralelas

b) Placa com quatro barras internas

O resultado para a flecha máxima das placas está mostrado na Tabela 4.13, onde o mesmo é comparado com o obtido com o programa PLENOR [160].

Tabela 4.13 - Flecha máxima para as placas do Exemplo 8.

\begin{tabular}{|c|c|c|}
\hline & MEC & PLENOR [160] \\
\hline Placa a) & 0,016 & 0,016 \\
\hline Placa b) & 0,013 & 0,012 \\
\hline
\end{tabular}




\section{CONSIDERAÇÕES FINAIS}

A finalidade básica deste trabalho foi apresentar uma formulação direta do Método dos Elementos de Contorno aplicado a placas com vinculação interna. A partir da teoria de Reissner, este objetivo foi alcançado incorporando-se ao sistema de equaçōes algébricas gerado pelo $M E C$, o enrijecimento produzido por esta vinculação.

Na formulação apresentada, o ponto de colocação para as equações

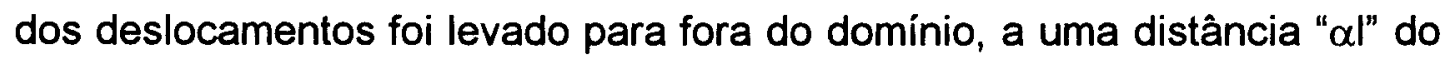
contorno. Esta alternativa simplificou a determinação dos coeficientes de influência, que foram calculados usando-se apenas esquemas de integração numérica. Conforme mostrado no exemplo 1 , os resultados dos deslocamentos e esforços para valores de " $\alpha$ " variando entre 0,10 e 1,50, mostraram uma precisão satisfatória quando comparados aos resultados analíticos obtidos por RIBEIRO [158]. Neste trabalho, adotou-se para " $\alpha$ " o valor igual a " 0,5 ", por estar dentro da faixa referida anteriormente e por seus resultados serem praticamente iguais aos obtidos com $\alpha=0,25$, valor recomendado por RIBEIRO [140]. Sabe-se no entanto, que os resultados seriam tanto melhores quanto mais próximo de zero ficasse o fator " $\alpha$ ", 0 que não é obtido numericamente, mesmo aumentando-se o número de pontos de integração. Este problema de condicionamento numérico das matrizes de influência é agravado quando a relação h/a é inferior a 0,01.

Deve-se salientar que este mal condicionamento das matrizes $\mathbf{H ~ e ~} \mathbf{G}$ gera grandes imprecisões apenas nos valores de contorno, ficando os valores de domínio com um bom nível de precisão. Consegue-se eliminar 
tais problemas dividindo-se o elemento que contenha o nó singular em subelementos, cujo comprimento é da mesma ordem de grandeza da distância que o separa do ponto de colocação. Obtém-se com esta técnica para valores de " $\alpha$ " da ordem de 0,001 , resultados com um ótimo nível de precisão, mesmo para valores de contorno.

Os resultados do exemplo 1 mostram ainda a importância da consideração da parcela da energia de deformação devida ao cisalhamento transversal, principalmente no que se refere aos deslocamentos transversais. A influência da espessura no cálculo destes, começa a ser evidenciada a partir, de relações h/a pequenas, chegando-se a um acréscimo de quase $40 \%$, para $h / a=0,30$, quando comparado com os resultados da Teoria Clássica, onde tal influência é desprezada.

A utilização da Teoria de Reissner possibilita a escolha de condições de contorno mais apropriadas no caso de bordas simplesmente apoiadas. Isto não acontece na Teoria Clássica, que admite apenas a condição "hard" de contorno, ou seja, são considerados nulos o deslocamento transversal, o momento normal ao contorno e, de forma aproximada, a rotação tangencial que é levada em conta através do conceito de força cortante equivalente. Além da condição "hard", a Teoria de Reissner admite a condição de contorno "soft" onde considera-se nulo o momento de torção ao contrário da rotação tangencial. Segundo RIBEIRO [140], esta condição deixa a placa mais flexivel, resultando deslocamentos e esforços ligeiramente superiores, aumentando-se esta diferença quando se aproxima dos apoios. Neste trabalho, quando não se escreveu em contrário, considerou-se condição "hard" de contorno.

Foram considerados carregamentos transversais atuantes em área de forma qualquer, com distribuição uniforme ou linear, cargas concentradas, cargas e momentos distribuidos em linha. A análise da aplicação de todos esses carregamentos tem a finalidade de atender ao cálculo de placas submetidas às mais variadas condições de carregamento, e de simultaneamente possibilitar a determinação do enrijecimento 
produzido pela vinculação interna, uma vez que o mesmo é equivalente às reações, concentradas ou distribuídas, que aparecerão na interface placaestrutura.

A análise de placas sobre apoios discretos foi desenvolvida considerando-se apoios pontuais e distribuídos em áreas. Os resultados com ambos os tipos de apoios mostraram-se praticamente coincidentes em termos de deslocamentos e esforços. Como era de se esperar, a diferença dos valores do momento fletor se acentua apenas sobre os apoios, uma vez que a singularidade existente nos apoios pontuais desaparece quando estes são considerados em uma área. Esta última situação é mais realista em termos de projeto, uma vez que os apoios têm uma área de interface, por menores que sejam as suas dimensões.

A associação da placa com uma estrutura qualquer foi levada em conta considerando-se que o enrijecimento produzido pela mesma era equivalente à aplicação de reações continuamente distribuídas em linhas, que são as barras da interface placa-estrutura. Estas reaçōes são na realidade linhas de momentos e de carga transversal distribuida em linha. Procurou-se desta forma evitar uma associação placa-estrutura em que o enrijecimento fosse obtido através de reações concentradas em pontos discretos, os nós da estrutura, não levando em conta a vinculação contínua produzida pelas barras.

Alguns exemplos de placas com apoios discretos e em linha foram apresentados e os resultados obtidos mostram que a utilização da técnica proposta tem um bom nivel de precisão.

Algumas sugestões para continuidade deste trabalho são relativas à consideração do comportamento plástico para o material da placa. Dividindo-se a espessura em camadas, seria possivel acompanhar o 
espalhamento do escoamento das fibras externas para as internas, o que possibilitaria a análise mais precisa de placas de materiais como o concreto armado. Poderia também ser incorporado à formulação, a análise de placas apoiadas em meio contínuo, possibilitando por exemplo, a análise de fundações tipo radier. 


\section{REFERÊNCIAS BIBLIOGRÁFICAS}

[1] SOUTHWELL, R.V. Relaxation methods in theoretical physics. London, Oxford University Press, 1946.

[2] TURNER, M.J. et al. Stifness and deflection analysis of complex structures. Journal Aero. Science, v. 23, p. 805-823, 1956.

[3] ARGYRIS, J.H.; KELSET, S. Energy theorems and structural analysis. London, Butterworths, 1960.

[4] CLOUGH, R.W. The finite element in plane stress analysis. In: ASCE CONF. ON ELETRONIC COMP., 2nd, 1960 - Proc.

[5] ZIENKIEWICZ, O.C. The finite element method in engineering science. London, McGraw-Hill, 1971.

[6] BREBBIA, C.A. The boundary element method for engineers. London, Pentech Press, 1978.

[7] VENTURINI, W.S. Boundary Element Method in Geomechanics. Berlin, Springer-Verlag, 1983.

[8] BREBBIA, C.A. ; TELLES, J.C.F. ; WROBEL, L. C. Boundary element techniques - Theory an applications in engineering. Berlin, Springer-Verlag, 1984.

[9] ANDERSSEN, R.S. et al. The application and numerical solution of integral equations. Alphen aan den Rijn, The Netherlands, Sijthoff \& Noordhoff, 1980.

[10] ABEL, N.H. Ouevres Complètes. Norvégien Christiania, v. 1, p. 621,1981

[11] GIL ROGRíGUEZ, J.C. Sobre o emprego do método dos elementos de contorno em problemas elásticos bidimensionais. São Carlos, 1986. Dissertação (Mestrado) - Escola de Engenharia, Universidade de São Paulo. 
[12] VOLTERRA, V. Opere mathematiche. Acad. Naz. Lincei, Rome, v. 2, p. 216-275, 1956.

[13] FREDHOLM, I. Sur une classe d'equations fonctionelles. Acta Math., v. 27, p. 365-390, 1903.

[14] HILBERT, D. Grundzüge einer allgemeinen theorie der linearen integralgleichungen. New York, Chelsea Publishing Company, 1953.

[15] KELLOG, O.D. Foundations of potencial theory. Berlim, SpringerVerlag, 1967.

[16 ] LOVE, A.E.H. A treatise on the mathematical theory of elasticity. 4th ed. New York, Dover, 1944.

[17] COSTA JR., J.A. The boundary element method applied to plate problems. Southampton, 1985. Thesis (Ph. D.) - University of Southampton.

[18] MUSKHELISHVILI, N.I. Some basic problems of the mathematical theory of elasticity. Groningen Holand, Noordhoff, 1953.

[19] MIKHLIN, S.G. Integral equations. London, Pergamon Press, 1957. (International series of monographs in pure an applied mathematics).

[20] KUPRADZE, V.D. Potencial methods in the theory of elasticity. Jerusalém, Israel Program for Scientific Translations, 1965.

[21] BANERJEE, P.K. Integral equation methods for analysis of piecewise non-homogeneus three-dimensional elastic solids of arbitrary shape. Int. Journal Mechanical Science, v. 18, p. 293303, 1976.

[22 ] JASWON, M.A. Integral equation methods in potential theory $I$. Proc. Royal Society, A 275, p. 23-32, 1963.

[23 ] SYMM, G.T. Integral equation methods in potencial theory II. Proc. Royal Society, A 275, p. 33-46, 1963.

[24] JASWON, M.A. ; PONTER, A.R. An integral equation solution of the torsion problem. Proc. Royal Society, A 273, p. 237-246, 1963.

[25] RIZZO, F.J. An integral approach to boundary value problems of classical elastostatics. Quarterly of Applied Mathematics, v. 25 (1) p. 83-92, 1967. 
[26 ] CRUSE, T.A. Numerical solutions in three dimensional elastostatics. Int. Journal of Solids and Structures, v. 5, p. 1259-1274, 1969.

[27] RICARDELLA, P.C. An implementation of the boundaryty and elasto-plasticity. 1973. Thesis ( Ph. D ) - Carnegie-Mellon University.

[28] CRUSE, T.A. An improved boundary integral equation method for three dimensional elastic stress analysis. Computers \& Structures, v. 4, p. 741-754, 1974.

[29] LACHAT, J.A. A further development of the boundary integral thechnique for elastostatics. Southampton, 1975. Thesis (Ph.D. ) - University of Southampton.

[30] JASWON, M.A. ; MAITI, M. ; SYMM, G.T. Numerical biharmonic analysis and some applications. Int. Journal of Solids and Structures, v. 3, p. 309-332, 1967.

[31] JASWON, M.A. ; MAITI, M. An integral formulation of plate bending problems. J. Engng. Math., v. 2, p. 83-93, 1968

[32] ALTIERO, N.J.; SIKARSKIE, D.L. A boundary integral method applied to plates of arbitrary plan form. Computer \& Structures , v. 9, p. 163-168, 1978.

[ 33 ] WU, B.C. ; ALTIERO, N.J. A boundary integral method applied to plates of arbitrary plan form and arbitrary boundary conditions. Computer \& Structures, v. 10, p. 703-707, 1979.

[34] TOTTENHAN, H. The boundary element method for plates and shells. In: BANERJEE, P.K. \& BUTTERFIELD, R. ed. Developments in boundary element methods. London, Elsevier, p. 173-205, 1979.

[35] BEZINE, G. Boundary integral formulation for plate flexure with arbitrary boundary conditions. Mechanics Research Communications, v. 5 (4), p. 197-206, 1978.

[ 36 ] BEZINE, G. ; GAMBI, D.A. A new integral equation formulation for plate bending problems. In: BREBBIA, C.A. ed.. Recent advances in boundary element methods, p. 327-342. London, Pentech Press, 1978.

[37] STERN, M. A general boundary integral formulation for the numerical solution of plate bending problems. Int. Journal of Solids and Structures, v. 15, p. 769-782, 1979. 
[ 38 ] STERN, M. Boundary integral equations for bending of thin plates. In: BREBBIA, C.A. ed.. Progress in boundary element methods , v. 2. London, Pentech Press, 1983.

[ 39 ] BEZINE, G. A mixed boundary integral: finite element approach to plate vibration problems. Mechanics Research Communications, v. 7 (3), p. 141-150, 1980.

[40] KAMIYA, N. et. al. An approximate finite deflection analysis of a heated elastic plate by the boundary element method. Appl. Math. Modelling, v. 6 (1), p. 23-27, 1982.

[ 41] KAMIYA, N. ; SAWAKY, Y. An integral equation approach to finite deflection of elastic plates. Int. J. Non-linear Mechanics, v. 17 (3), p. 187-194, 1982.

[ 42 ] TANAKA, M. Large deflection analysis of thin elastic plates. In: BANERJEE, P.K. ; MUKHERJEE, S. ed.. Developments in boundary element methods, v. 3. London, Elsevier, 1984.

[ 43 ] WEEËN, F.V. Application of the direct boundary element method to Reissner's plate model. In: BREBBIA, C.A. ed.. Boundary element methods in engineering: Procedings of the Fourth International Seminar, Southampton, England, Sept. 1982.

[ 44 ] REISSNER, E. On the theory of bending of elastic plates. J. Math. Physics, v. 23, p. 184-191, 1944.

[ 45] REISSNER, E. The effect of transverse shear deformation on the bending of elastic plates. Journal of Applied Mechanics, v. 12 p.A69-A77, 1945.

[ 46 ] REISSNER, E. On bending of elastic plates. Quart. Appl. Math., v.5(1), p. 55-68, 1947.

[47] KATSIKADELIS, J.T. ; ARMENÀKAS, A.E. Plates on elastic foundations by BIE method. Journal of Engineering Mechanics, ASCE, v. 110 (7), p. 1086-1104, 1984.

[ 48 ] KATSIKADELIS, J.T. ; ARMENÀKAS, A.E. Analysis of clamped plates on elastic foundation by the boundary integral method. Journal of Applied Mechanics, v. 54, p. 544-580, 1984.

[ 49 ] WINKLER, E. Die Lehre von der Elastizitaet und Festigkeit. Piague, Dominicus, 1867.

[50] COSTA JR., J.A. ; BREBBIA, C.A. The boundary element method 
applied to plates on elastic foundations. Engineering Analysis, v.2 (4), p. 174-183, 1985.

[51] COSTA JR., J.A. ; BREBBIA, C.A. On the reduction of domain integrals to the boundary for the BEM formulation of plates on elastic foundations. Engineering Analysis, v. 3 (2), p. 123-126, 1986.

[ 52 ] COSTA JR., J.A. ; BREBBIA, C.A. Bending of plates on elastic foundations using the boundary element method. In: INTERNATIONAL CONFERENCE, 2nd, Southampton, 1985 Proc.. Berlin, Springer-Verlag, 1985.

[53] SILVA, N.A. Aplicação do método dos elementos de contorno à análise de placas sobre fundações elásticas. São Carlos, 1988. Dissertação (Mestrado) - Escola de Engenharia de São Carlos, Universidade de Săo Paulo.

[54] SILVA, N.A. ; VENTURINI, W.S. Aplicação do método dos elementos de contorno na determinação de cargas últimas em sapatas isoladas. In: MECON 90. Anais. p. C29-C38, 1990.

[55] BREBBIA, C.A. ; NARDINI, D. Solution of parabolic and hiperbolic time dependent problems using boundary elements. Comp. \& Maths. with Appls., v. 12B, p. 1061-1072, 1986.

[56] MENDELSON, A. Boundary integral methods in elasticity and plasticity. NASA report TN D - 7418, 1973.

[57] MUKHERJEE, S. Corrected boundary integral equation in planar thermo elastoplasticity. Int. Journal of Solids and Structures, v. 13, p. 331-335, 1977.

[ 58] CHAUDONNERET, M. Méthode des équations intégrales appliqués a la résolution des problèmes de visco-plasticité. Journal Méchanique Appliqueé, v. 1, p. 113-132, 1977.

[59] MORJARIA, M. ; MUKHERJEE, S. Inelastic analysis of transverse deflection of plates by boundary element method. Journal of Applied Mechanics, v. 47 (2), p. 291-296, 1980.

[60] TELLES, J.C.F. ; BREBBIA, C.A. New developments in elastoplastic analysis. In: BREBBIA, C.A. ed.. Boundary element methods. Berlin, Springer-Verlag, 1981.

[61] VENTURINI, W.S. ; BREBBIA, C.A. The boundary element method for the solution of no-tension materials. In: BREBBIA, C.A. ed.. 
Boundary element methods. Berlin, Springer-Verlag, 1981.

[62 ] VENTURINI, W.S. Aplication of the boundary element method to solve geomechanical problems. Southampton, 1982. Thesis(Ph.D.) - University of Southampton.

[63 ] MAIER, G. ; POLIZZOTTO, C. A boundary element approach to limit analysis. In: BREBBIA, C.A. et. al. ed.. Boundary elements $V$. Berlin, Springer-Verlag, 1983.

[64] TSUTA, T. ; YAMAJI, S. Boundary element analysis of contact thermo-elastoplastic problems with creep and the numerical technique. In: BREBBIA, C.A. et. al. ed.. Boundary elements $V$. Berlin, Springer-Verlag, 1983.

[65] TAKAKUDA, K. ; KOIZUMI, T. ; SHIBUYA, T. On integral equation methods for crack problems. Bulletin of JSME, v. 28 (236), p. 217-224, 1985.

[66 ] ZIENKIEWICZ, O.C. ; KELLY, D.W. ; BETESS, P. The coupling of the finite element method and boundary solution procedures. International Journal for Numerical Methods in Engineering, v. 11, p. $355-375,1977$.

[67] ZIENKIEWICZ, O.C. The finite element method. London, McGrawHill, 1971.

[68 ] BEER, G. Finite element, boundary element and coupled analysis of unbounded problems in elastostatics. International Journal for Numerical Methods in Engineering, v. 19, p. 567-580, 1983.

[69] BREBBIA, C.A ; WALKER, S. Boundary element thechniques in engineering. London, Butterworths, 1980.

[70] FELIPPA, C.A. Interfacing finite element and boundary element discretizations. In: BREBBIA, C.A. ed.. Boundary element methods. Berlin, Springer-Verlag, 1981.

[71] BEER, G. ; MEEK, J.L. The coupling of boundary and finite element methods for infinite domain problems in elasto-plasticity. In: BREBBIA, C.A. ed.. Boundary element methods. Berlin, Springer-Verlag, 1981.

[72] DENDROU, B.A. ; DENDROU, S.A. A finite element-boundary integral scheme to simulate rock effects on the linear of an underground intersection. In: BREBBIA, C.A. ed.. Boundary element methods. Berlin, Springer-Verlag, 1981. 
[73 ] BRADY, B.H.G. ; WASSYNG, A. A coupled finite element-boundary element method of stress analysis. International Journal of Rock Mechanics, Mining Science \& Geomechanics Abstracts , v. 18, p. $475-485,1981$.

[ 74] KISHIMOTO, K. et. al. Elastic-plastic fracture mechanics analysis by combination of boundary and finite element methods. In: BREBBIA, C.A. et. al. ed.. Boundary elements $V$. Berlin, Springer-Verlag, 1983.

[75] HISATAKE, M. ; ITO, T. ; UEDA, H. Three dimensional symmetric coupling of boundary and finite element methods. In: BREBBIA, C.A. et. al. ed.. Boundary element $V$. Berlin, Springer-Verlag, 1983.

[76] ICHIKAWA, Y.; OBARA, Y.; KAWAMOTO, T. A coupling scheme for boundary and finite elements using a joint element. Int. Journal for Numerical and Analytical Methods in Geomechanics, v. 9, p. 161-172, 1985.

[77] PAULA, F. A. Obtenção de matriz de rigidez utilizando o método dos elementos de contorno. Rio de Janeiro, 1986. Dissertação (Mestrado) - Universidade Federal do Rio de Janeiro, COPPE.

[78 ] KIRCHHOFF, G. Über das Gleichgewicht und die Bewegung Einer Elastischen Scheibe. J. Math. Crelle, v. 40, p. 51-58, 1850.

[79] MINDLIN, R.D. Influence of rotatory inertia and shear on flexural motions of isotropic elastic plates. J. Appl. Mech. , v. 13 (1), p. 31-38, 1951.

[80] REISSNER, E. On the theory of transverse bending of elastic plates. Int. J. Solids Structures, v. 12 (8A), p. 545-554, 1976.

[81] SALERNO, V. L. ; GOLDBERG, M.A. Effect of shear deformations on the bending of retangular plates. J. Appl. Mech., p. 54-58, 1960.

[82] PANC, V. Theories of elastic plates. International Publishing, Noordhoff, 1975.

[83 ] LEVINSON, M. An accurate, simple theory of statics and dynamics of elastic plates. Mech. Research Communications, v. 7 (6), p. 343-350, 1980.

[84] REISSNER, E. On small deflections of sheardeformable elastic plates. Comput. Meth. Appl. Mech. Eng., v. 59, p. 227-233, 1986. 
[85] REISSNER, E. On a generalization of some formulas of the theory of moderately thick elastic plates. Int. J. Solids Structures, v. 23 (6), p. 711-717, 1987.

[ 86 ] REISSNER, E. On the asymptotic expansions for the sixth-order linear theory problem of transverse bending of orthotropic elastic plates. Comput. Meth. Appl. Mech. Eng., v. 85 (1), p. 75-88, 1991.

[ 87 ] NORDGREN, R.P. A bound on the error in plate theory. Quart. Appl. Math., v. 28 (4), p. 587-595, 1971.

[88 ] NORDGREN, R.P. A bound on the error in Reissner's theory of plates. Quart. Appl. Math., v. 29, p. 551-556, 1972.

[89] RYCHTER, Z. A sixth-order plate theory derivation and error estimates. J. Appl. Mech., v. 54 (2), p. 275-279, 1987.

[90] RYCHTER, Z. An improved error estimate for Reissner's plate theory. Int. J. Solids Structures, v. 24 (5), p. 537-544, 1988.

[91] LADEVĖZE, P. ; PECASTAINGS, F. The optimal version of Reissner's theory. J. Appl. Mech., v. 55 (2), p. 413-418, 1988.

[ 92 ] BARRET, K.E. ; ELLIS, S. An exact theory of elastic plates. Int. J. Solids Structures, v. 24 (9), p. 859-880, 1988.

[93] BATOZ, J.L. ; BATHE, K.J. ; HO, L.W. A study of three-node triangular plate bending elements. Int. J. Num. Meth. Eng., v. 15, p.1771-1812, 1980.

[94 ] HRABOK, M.M. ; HUDEY, T.M. A review and catalogue of plate bending finite elements. Comput. \& Structures, v. 19, p. 479-498, 1984.

[95] BAZELEY, G.P. ; CHEUNG, Y.K. ; IRONS, B.M. ; ZIENKIEWICZ, O.C. Triangular elements in plate bending conforming and nonconforming solutions. In: CONF. ON MATRIX METHODS IN STRUCTURAL MECHANICS, WPAFB, Proc.. Ohio, 1965. p. 547576.

[96 ] PIAN, T.H.H. Derivation of element stiffness matrices by assumed stress distributions. AlAA J., v. 2, p. 133-1333, 1964.

[97 ] BATOZ, J.L. An explicit formulation for an efficient triangular plate bending element. Int. J. Num. Meth. Eng., v. 18, p. 1077-1089, 1982 
[98] HUGHES, T.J.R. The finite element method - linear, static and dynamic finite element analysis. New Jersey, Prentice Hall, Englewood Cliffs, 1987.

[ 99] TESSLER, A. ; HUGHES, T.J.R. A three-node Mindlin plate element with improved transverse shear. Comp. Meth. Appl. Eng., v. 50 , p. 71-101, 1985.

[100] PAPADOPOULOS, P. ; TAYLOR, R.L. A triangular element based on Reissner-Mindlin plate theory. Int. J. Num. Meth. Eng., v. 30 , p.1029-1049, 1990.

[101] ZIENKIEWICZ, O.C. ; LEFEBVRE, D. A robust triangular plate bending element of the Reissner - Mindlin type. Int. J. Num. Meth. Eng., v. 26, p. 1169-1184, 1988.

[102] HÄGGBLAD, B. ; BATHE, K.J. Specifications of boundary conditions for Reissner-Mindlin plate bending finite elements. Int. J. Num. Meth. Eng., v. 30 (5), p. 981-1011, 1990.

[103] BERGAN, P.G. ; HANSSEN, L. A new approach for deriving good element stiffness matrices. In: WHITEMAN, J.R. ed.. The mathematics of finite elements and applications II. London, Academic Press, 1975. p. 483-498.

[104] BERGAN, P.G. ; NYGARD, M.K. Finite elements with increased freedom in choosing shape functions. Int. J. Num. Meth. Eng. , v.20, p. 643-663, 1984.

[105] BERGAN, P.G. ; NYGARD, M.K. Non-linear shell analysis using free formulation finite elements. In: BERGAN, P.G. ; BATHE, K.J. ; WUNDERLICH, ed. Finite elements for non-linear problems. Berlin, Springer-Verlag, 1985. p. 317-338.

[106] NYGARD, M.K. ; BERGAN, P.G. Nonconforming finite elements based on the free formulation. In: KUHN, G. ; MANG, H. ed.. Discretization methods in structural mechanics. Berlin, SpringerVerlag, 1989. p. 71-82.

[107] FELLIPA, C.A. ; BERGAN, P.G. A triangular bending element based on energy - orthogonal free formulation. Comput. Meth. Appl. Mech. Eng., v. 61, p. 129-160, 1987.

[108] BERGAN, P.G. ; FELIPPA, C.A. A triangular membrane element with rotational degrees of freedom. Comput. Meth. Appl. Mech. Eng. , v. 50, p. 25-69, 1985. 
[109] HANSEN, E.B. Numerical solution of integro-differential and singular integral equations for plate bending problems. Journal of Elasticity, v. 6 (1), p. 39-56, 1976.

[110] GUOSHU, S. ; MUKERJEE, S. Boundary element method analysis of bending plates of arbitrary shape with general boundary conditions. Eng. Analysis, v.3 (1), p. 36-44, 1986.

[111] HARTMANN, F. ; ZOTENANTEL, R. The direct boundary element method in plate bending. Int. Num. Meth. Eng., v. 23 (11), p.2049-2069, 1986.

[112] ABRAMOWITZ, M. ; STEGUN, I.A. Handbook of mathematical functions. New York, Dover Publications, 1965.

[113] PAIVA, J.B. Formulação do método dos elementos de contorno para a flexão de placas e suas aplicações em engenharia de estruturas. São Carlos, 1987. Tese (Doutorado) - Escola de Engenharia de São Carlos, Universidade de São Paulo.

[114] ABDEL-AKHER, A. ; HARTLEY, G.A. Analytic integration procedures for plate bending analysis. In: BREBBIA, C.A. ed. Boundary elements X. 1988. C. M. Publ.. v. 3, p. 391-405.

[115] AHMED, A.A. ; HARTLEY, G.A. Evaluation of boundary integrals for plate bending. Int. J. Num. Meth. Eng., v. 28 (2), p. 75-93, 1989.

[116] ABDEL-AKHER, A. ; HARTLEY, G.A. Boundary integration and interpolation procedures for plate bending. Int. J. Num. Meth. Eng., v. 28 (6), p. 1389-1408, 1989.

[117] HARTMANN, F. Kirchhoff plates. In: BREBBIA, C.A. ed.. Boundary elements $X$. 1988. C. M. Publ.. v. 3, p.409-423.

[118] PILTNER, R. ; TAYLOR, R.L. The solution of plate bending problems with the aid of a boundary element algorithm based on singular complex functions. In: TANAKA, M. ; BREBBIA, C.A. ; HONMA, T. ed.. Boundary elements XII, 1989.

[119] KATSIKADELIS, J.T. ; ARMENAKAS, A.E. A new boundary equation solution to the plate problem. J. Appl. Mech., v. 56 (2), p. 364374, 1989.

[120] SAPOUNTZAKIS, E.J. ; KATSIKADELIS, J.T. Boundary element solution of plates of variable thickness. J. Eng. Mech., v. 117 (6), p. 1241-1256, 1991. 
[121] VITOORAPORN, C. ; MOSHAIOV, A. Effectiveness study of higher order elements in the BIE method for thin elastic plate bending. In: TANAKA, M. ; BREBBIA, C.A. ; HONMA, T. ed.. Boundary elements XII, 1989.

[122] BEZINE, G.P. A new BEM for bending of plates on elastic foundations. Int. J. Solids Structures, v. 24 (6), 557-565, 1988.

[123] KATSIKADELIS, J.T. ; KALLIVOKAS, L.F. Clamped plates on pasternak - type elastic foundation by BEM. J. Appl. Mechanics, v. 53 (4), p. 909-917, 1986.

[124] KATSIKADELIS, J.T. ; KALLIVOKAS, L.F. Plates on biparametric elastic foundation by BIDE method. J. Eng. Mech., v. 114 (5), p.847-875, 1988.

[125] KATSIKADELIS, J.T. Large deflection analysis of plates on elastic foundation by the boundary element method. Int. J. Solids Structures, v. 27 (15), p. 1867-1878, 1991.

[126] TANAKA, $M$. Integral equation approach to small and large displacements of thin elastic plates. In: BREBBIA, C.A. ed.. Boundary element method in engineering. Proc. 4th. Int. Seminar, Heildeberg, Springer-Verlag, 1982.

[127] KAMIYA, N. ; SAWAKI, Y. Finite deflection of plates. In: BREBBIA, C.A. ed.. Topics in boundary element research. Heildeberg, Springer-Verlag, 1984.

[128] YE, T.Q. ; LIN, Y. Finite deflection analysis elastic plate by the boundary element method. Appl. Math. Modelling, v. 9, p.183188, 1985.

[129] SAWAKI, Y.; TAKEUCHI, K. ; KAMIYA, N. Finite deflection analysis of plates by dual reciprocity boundary elements. In: BREBBIA, C.A. ; CONNOR, J. ed.. Advances in boundary elements - vol. 3 Stress analysis. Springer-Verlag, 1989.

[130] KATSIKADELIS, J.T. ; NERANTZAKI, M.S. Large deflections of thin plates by BEM. In: BREBBIA, C.A. ed.. Boundary elements $X$. 1988.

[131] KAMIYA, N. ; SAWAKI, Y. ; NAKAMURA, Y. Boundary element nonlinear bending analysis of clamped sandwich plates and shells. In: BREBBIA, C.A. ed.. Proc. of 4th Int. Seminar., Southampton, 1982. p. $515-525$. 
[132] KAMIYA, N. Structural non-linear analysis by boundary element methods. In: BREBBIA, C.A. ed.. Boundary elements $X$ - vol. 3 Stress analysis, 1988.

[133] PROVIDAKIS, C.P. ; BESKOS, D.E. Free and forced vibrations of plates by boundary elements. Comput. Meth. Appl. Mech. Eng. , v.74 (3), p. 231-250, 1989.

[134] PROVIDAKIS, C.P. ; BESKOS, D.E. Free and forced vibrations of plates by boundary and interior elements. Int. J. Num. Meth. Eng., v. 28 (9), p. 1977-1994, 1989.

[135] AKKARI, M.M. ; HUTCHINSON, J.R. Boundary point collocation method for the vibration of a Mindlin thick plate. In: BREBBIA, C.A. ed.. Boundary elements $X$ - vol. 3 - Stress analysis, 1988. p. 465-476.

[136] MORJARIA, M. ; MUKERJEE, S. Numerical analysis of planar time dependent inelastic deformation of plates with crack by the boundary element method. Int. J. Solids Structures, v. 17, p. 127, 1981.

[137] MUKERJEE, S. ; MORJARIA, M. A boundary element analysis of time dependent inelastic deformation of cracked plates loaded in antiplane shear. Int. J. Solids Structures, v. 17, p. 253- , 1981.

[138] MUKERJEE, S. ; MORJARIA, M. A boundary element formulation for planar time dependent inelastic deformation of plates with cutouts. Int. J. Solids Structures, v. 17, p. 115- , 1981.

[139] MOSHAIOV, A. ; VORUS, W. S. Elasto-plastic bending analysis by a boundary element method with initial plastic moments. Int. J. Solids Structures, v. 22 (11), p. 1213-1229, 1986.

[140] RIBEIRO, G.O. Sobre a formulação do método dos elementos de contorno para a flexão de placas usando as hipóteses de Reissner. São Carlos, 1992. Tese (Doutorado) - Escola de Engenharia de São Carlos, Universidade de São Paulo.

[141] COSTA JR., J. A. ; BREBBIA, C.A. Elastic buckling of plates using the boundary element method. In: BREBBIA, C.A. ; MAIER, G. ed.. Boundary elements VII. Proc. 7th. Int. Conf. Berlin, Springer-Verlag, 1985.

[142] BEZINE, G.P. ; CIMETIERRE, A. ; GELBERT, J.P. Unilateral buckling of thin elastic plates by the boundary integral equation method. Int. J. Num. Meth. Eng., v. 21, p. 2189-2199, 1985. 
[143] WEEËN, F.V. Application of boundary integral equation method to Reissner's plate model. Int. J. Num. Meth. Eng., v. 18 (1), p. 110, 1982.

[144] KARAM, V.J. Aplicação do método dos elementos de contorno à teoria de Reissner para flexão de placas. Rio de Janeiro, 1986. Dissertação (Mestrado) - Universidade Federal do Rio de Janeiro, COPPE.

[145] RIBEIRO, G.O. ; VENTURINI, W.S. Aplicação do método dos elementos de contorno no cálculo de placas através da teoria de Reissner. MECOM-89 - Anais do X Congresso Ibero-LatinoAmericano sobre Métodos Computacionais em Engenharia - II Encontro Nacional de Mecânica Computacional. Porto, Portugal, 1989.

[146] BARCELLOS, C.S. ; SILVA, L.H.M. A boundary element formulation for the Mindlin's plate model. In: BREBBIA, C.A. ; VENTURINI, W.S. ed. Boundary element tecniques: applications in stress analysis and heat transfer. Southampton, 1987. C. M. Publ.

[147] WESTPHAL JR., T. ; BARCELLOS, C.S. Applications of the boundary element method to Reissner's and Mindlin's plate models. In: TANAKA, M. ; BREBBIA, C.A. ; HONMA, T. ed.. Boundary elements XII, 1989.

[148] XIAO-YAN, L. ; KUANG-MAO, H. ; XIUXI, W. Geometrically nonlinear analysis of a Reissner type plate by the boundary element method. Comp. \& Structures, v. 37 (6), p. 911-916, 1990.

[149] NG, S.F. ; CHEUNG, M.S. ; XU, T. A combined boundary element and finite element solution of slab and slab-on-girder bridges. Comp. \& Structures, v. 37 (6), p. 1069-1075, 1990.

[150] NAKAGUMA, R.K. Three dimensional elastostatics using the boundary element method. Southampton, 1979. Thesis (Ph. D.) University of Southampton.

[151] CUROTTO, C. L. Método dos elementos de contorno para elasticidade tridimensional. Rio de Janeiro, 1981. Dissertação (Mestrado) - Universidade Federal do Rio de Janeiro, COPPE.

[152] SÁ, P.A.C.O. ; TELLES, J.C.F. Análise de problemas de elasticidade linear tridimensional pelo método dos elementos de contorno utilizando as soluções fundamentais de Kelvin e Mindlin. In: CONGRESSO LATINO-AMERICANO SOBRE MÉTODOS COMPUTACIONAIS PARA ENGENHARIA, 7., São Carlos, 1986. 
Anais. Escola de Engenharia de São Carlos - Universidade de São Paulo.

[153] BARBIRATO, J.C.C. Formulação do método dos elementos de contorno para sólidos elásticos tridimensionais, baseada na solução fundamental de Mindlin. São Carlos, 1991. Dissertação (Mestrado) - Escola de Engenharia de São Carlos, Universidade de São Paulo.

[154] BANERJEE, P.K. A contribution to the study of axially loaded pile foundations. Southampton, 1969. Thesis (Ph. D. ) - University of Southampton.

[155] BUTTERFIELD, R. ; BANERJEE, P.K. The elastic analysis of compressible piles and pile groups. Geotechnique, v. 21, p. 4360, 1971.

[156] TIMOSHENKO, S. P. ; WOYNOWSKY-KRIEGER, S. Theory of plates and shells. New York, Mc Graw Hill Book Company, Inc., 1959.

[157] MARTINELLI, D.A.O ; MONTANARI, I. ; SAVASSI, W. Placas elásticas - Publicação 026/86 - Escola de Engenharia de São Carlos - USP - 1986.

[158] RIBEIRO, J.R.M. Estudo da validade da hipótese de Kirchhoff-Love na teoria de placas. Rio de Janeiro, 1976. Dissertação (Mestrado) - Universidade Federal do Rio de Janeiro, COPPE.

[159] BATHE, K.J. ; LEE, N.S. ; BUCALEM, M.L. On the use of hierarchical models in engineering analysis. Comp. Meth. Appl. Eng., v. 82, p.5-26, 1990.

[160] CAMPOS, M.R. Projeto e implementação de um software amigável para análise de placas ortótropas com enrijecedores. Belo Horizonte, 1996. Dissertação (Mestrado) - Universidade Federal de Minas Gerais, UFMG. 


\section{APÊNDICE A}

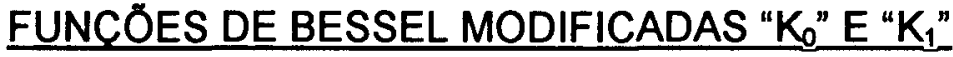

As funçōes de Bessel modificadas de ordem inteira " $k_{0}(z)$ " e " $k_{1}(z)$ " podem ser calculadas pelas seguintes expansōes polinomiais, dadas por ABRAMOWITZ [112], sendo " $z$ " um argumento real:

a) para $0<z \leq 2$ :

$$
\begin{aligned}
& k_{0}(z)=-\ln \left(\frac{z}{2}\right) I_{0}(z)-0,57721566+0,42278420\left(\frac{z}{2}\right)^{2}+ \\
& 0,23069756\left(\frac{z}{2}\right)^{4}+0,03488590\left(\frac{z}{2}\right)^{6}+0,00262698\left(\frac{z}{2}\right)^{8}+ \\
& 0,00010750\left(\frac{z}{2}\right)^{10}+0,00000740\left(\frac{z}{2}\right)^{12}+\varepsilon \\
& |\varepsilon|<1 \times 10^{-8}
\end{aligned}
$$




$$
\begin{aligned}
& k_{1}(z)=\frac{1}{z}\left[z \ln \left(\frac{z}{2}\right) I_{1}(z)+1+0,15443144\left(\frac{z}{2}\right)^{2}-0,67278579\left(\frac{z}{2}\right)^{4}-\right. \\
& 0,18156897\left(\frac{z}{2}\right)^{6}-0,01919402\left(\frac{z}{2}\right)^{8}-0,00110404\left(\frac{z}{2}\right)^{10}- \\
& \left.0,00004686\left(\frac{z}{2}\right)^{12}+\varepsilon\right] \\
& |\varepsilon|<8 \times 10^{-8}
\end{aligned}
$$

onde:

$$
\begin{aligned}
& I_{0}(z)=1+3,5156229 t^{2}+3,0899424 t^{4}+1,2067492 t^{6}+ \\
& 0,2659732 t^{8}+0,0360768 t^{10}+0,0045813 t^{12}+\varepsilon \\
& |\varepsilon|<1,6 \times 10^{-7} \\
& I_{1}(z)=z\left[0,5+0,87890594 t^{2}+0,51498869 t^{4}+0,15084934 t^{6}+\right. \\
& \left.0,02658733 t^{8}+0,00301532 t^{10}+0,00032411 t^{12}+\varepsilon\right] \\
& |\varepsilon|<8 \times 10^{-9}
\end{aligned}
$$

sendo:

$$
t=\frac{z}{3,75}
$$


b) para $z \geq 2$

$$
\begin{aligned}
& k_{0}(z)= \frac{1}{\sqrt{z} e^{2}}\left[1,25331414-0,07832358\left(\frac{2}{z}\right)+0,02189568\left(\frac{2}{z}\right)^{2}-\right. \\
& 0,01062446\left(\frac{2}{z}\right)^{3}+0,00587872\left(\frac{2}{z}\right)^{4}-0,00251540\left(\frac{2}{z}\right)^{5}+ \\
&\left.0,00053208\left(\frac{2}{z}\right)^{6}+\varepsilon\right] \\
&|\varepsilon|<1,9 x 10^{-7} \\
& k_{1}(z)= \frac{1}{\sqrt{z} e^{z}}\left[1,25331414+0,23498619\left(\frac{2}{z}\right)-0,03655620\left(\frac{2}{z}\right)^{2}+\right. \\
& 0,01504268\left(\frac{2}{z}\right)^{3}-0,00780353\left(\frac{2}{z}\right)^{4}+0,00325614\left(\frac{2}{z}\right)^{5}- \\
&|\varepsilon|<2,2 \times 10^{-7} \\
&\left.0,00068245\left(\frac{2}{z}\right)^{6}+\varepsilon\right]
\end{aligned}
$$

São ainda definidas:

$$
\begin{aligned}
& A(z)=k_{0}(z)+\frac{2}{z}\left[k_{1}(z)-\frac{1}{z}\right] \\
& B(z)=k_{0}(z)+\frac{1}{z}\left[k_{1}(z)-\frac{1}{z}\right]
\end{aligned}
$$


As derivadas das expressões (A.8) são:

$$
\begin{aligned}
& A^{\prime}(z)=-k_{1}(z)-\frac{2}{z^{2}}\left[k_{1}(z)-\frac{1}{z}\right]-\frac{2}{z}\left[k_{0}(z)+\frac{k_{1}(z)}{z}-\frac{1}{z^{2}}\right] \\
& B^{\prime}(z)=-k_{1}(z)-\frac{1}{z^{2}}\left[k_{1}(z)-\frac{1}{z}\right]-\frac{1}{z}\left[k_{0}(z)+\frac{k_{1}(z)}{z}-\frac{1}{z^{2}}\right]
\end{aligned}
$$

Rearranjando-se (A.9), tem-se:

$$
\begin{aligned}
& A^{\prime}(z)=-k_{1}(z)-\frac{2}{z}\left[k_{0}(z)+\frac{2}{z} k_{1}(z)-\frac{2}{z^{2}}\right] \\
& B^{\prime}(z)=-k_{1}(z)-\frac{1}{z}\left[k_{0}(z)+\frac{2}{z} k_{1}(z)-\frac{2}{z^{2}}\right]
\end{aligned}
$$

Substituindo-se a primeira das equações (A.8) em (A.10), obtém-se:

$$
\begin{aligned}
& A^{\prime}(z)=-\frac{1}{z}\left[z k_{1}(z)+2 A(z)\right] \\
& B^{\prime}(z)=-\frac{1}{z}\left[z k_{1}(z)+A(z)\right]
\end{aligned}
$$

